\section{AEC RESEARCH and} DEVELOPMENT REPORT

\title{
EXPERIMENTAL INVESTIGATION OF THE TURBULENCE STRUCTURE IN THE LOWER ATMOSPHERE
}

C. E. ELDERKIN

DECEMBER, 1966

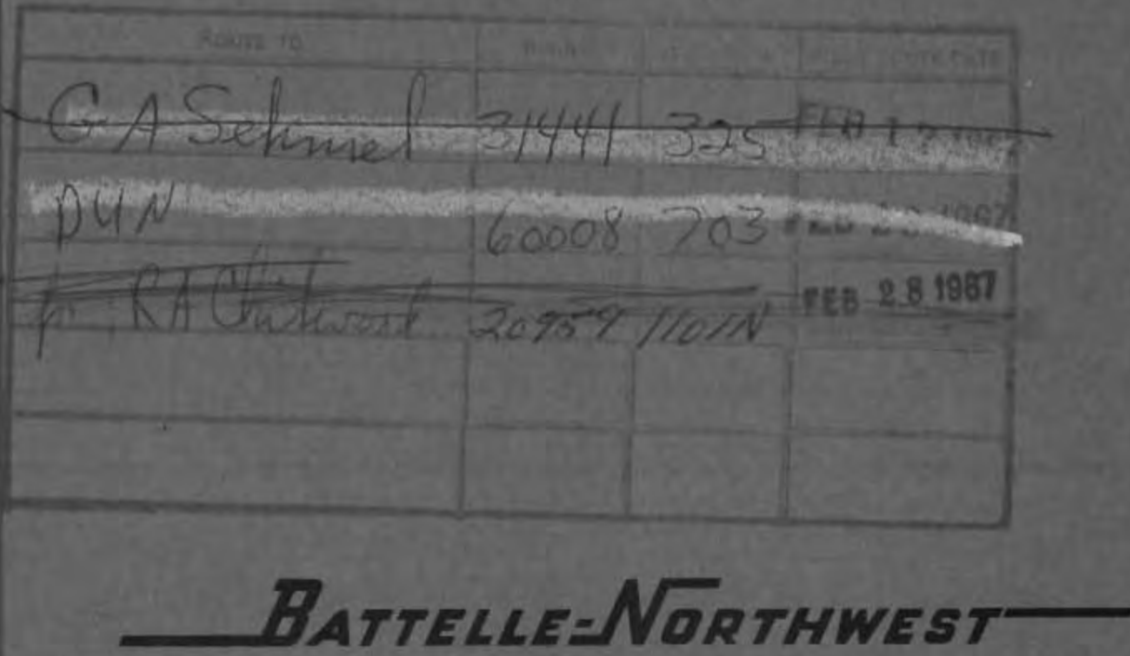

BATTELLE MEMORIAL INSTITUTE / PACIFIC NORTHWEST LABORATORY 


\section{LEGAL NOTICE}

This report was prepared as an account of Government sponsored work. Neither the United States, nor the Commission, nor any person acting on behalf of the Cammission:

A. Makes any warranty or representation, expressed or implied, with respect to the accuracy, completeness, or usefulness of the information contoined in this report, or that the use of any information, apparatus, method, or process disclosed in this report may not infringe privately owned rights; or

B. Assumes any liabilities with respect to the use of, or for damages resulting from the use of any information, apparatus, method, or process disclosed in this report.

As used in the above, "person acting on behalf of the Commission" includes any employee or contractor of the Commission, or employee of such contractor, to the extent that such employee or contractor of the Commission, or employee of such contractor prepares, disseminates, or provides access to, any information pursuant to his employment or contract with the Commission, or his employment with such contractor.

PACIFIC NORTHWEST LABORATORY

RICHLAND, WASHINGTON

operated by

BATTELLE MEMORIAL INSTITUTE

for the

UNITED STATES ATOMIC ENERGY COMMISSION UNDER CONTRACT AT(45-1)-1830

•

PRINTED BY/FOR THE U, S, ATOMIC ENEHOY COMMisston 
BNWL - 329

UC - 53 Meteorology

\title{
EXPERIMENTAL INVESTIGATION OF THE TURBULENCE STRUCTURE IN THE LOWER ATMOSPHERE
}

\author{
C. E. Elderkin \\ Atmospheric Sciences Section \\ Environmental and Radiological Sciences Department
}

December 1966

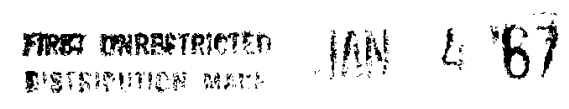


.

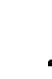

,

Printed in USA. Price \$5.00. Available from the Clearinghouse for Federal Scientific and Technical Information National Bureau of Standards U.S. Department of Commerce Springfield, Virginia 22151 


\author{
Abstract \\ EXPERIMENTAL INVESTIGATION OF THE TURBULENCE STRUCTURE \\ IN THE LOWER ATMOSPHERE \\ bY CHARLES EDWIN ELDERKIN
}

Measurements of turbulence in all three components of the wind were made at several heights, in neutral, unstable, and stable conditions, and in a variety of wind speeds. The data were analyzed on an analog computer for power spectral density distributions, cospectral density distributions and Reynolds' stresses. Similarity of the power spectral distributions was observed for each of the components with some notable deviations. The spectra for the vertical component measured at 12 meters and above, where bouyancy becomes effective, demonstrated definite convective energy peaks. These occurred in addition to the mechanical energy peaks which corresponded in the normalized spectral distributions to those for the tests close to the ground where mechanical energy dominated the structure.

A shift to higher wave numbers was detected for stable cases in the spectra for all three components. The shift was most noticeable at 12 meters where greater stabilities were more 
possible than close to the qround.

The spectral function for the inertial subrange, described by Kolmogoroff's relation, $S(x) \propto \varepsilon^{2 / 3} k^{-5 / 3}$, was detected consistently in the longitudinal and vertical component spectra. Reasonable values resulted for the universal porportionality constant, a, estimated best by $a=.51$, and dependence on $\varepsilon^{2 / 3}$ was demonstrated. The additional factor of $4 / 3$ required between the longitudinal and vertical one-dimensional spectra was missing, however. Only two cases for the lateral wind component showed a minus five-thirds dependence. The additional reauirement for the inertial subrange, that the cospectra become zero, was observed to hold only down to $n z / \bar{U}=1.0$ to 3.0 while the minus five-thirds law for the power spectra extended somewhat below this value. 
TABLF OF CONTENTS

Page

I. INTRODUCTION . . . . . . . . . . . . . . . . . 1

II. EQUIPMENT . . . . . . . . . . . . . . . . . . 5

Turbulence Sensing Equipment . . . . . . . 5

Recording Equipment .. . . . . . . . . 11

Analysis Equipment . . . . . . . . . . . 14

III. THEORETICAL BACKGROUND FOR ANALYSIS . . . . . . 33

IV. DATA ANALYSIS PROGRAMS . . . . . . . . . . . 37

Coordinate Transformation Program . . . . . 37

Reynolds' Stress Program . . . . . . . . . 41

Power Spectral Program . . . . . . . . . 44

Cospectral Program . . . . . . . . . . 50

Alternate Spectral Density Program . . . . . . 52

Statistical Reliability of Spectral Estimates • 53

V. FIELD TESTS . . . . . . . . . . . . . . 72

VI. THEORETICAL BACKGROUND FOR TURBULENCE RESULTS • - 76

VII. RESULTS OF ANALYSIS . . . . . . . . . . . . . 90

Longitudinal Component Spectra . . . . . . 90

Vertical Component spectra . . . . . . . . 92

Lateral Component Spectra . . . . . . . 95

The Universal Constants of the Inertial

Subrange ............. 101

Comparison with Previous Results . . . . . . 104

Reynolds'stress Results . . . . . . . . . 114 
VIII. SUMMARY AND CONCLUSIONS . . . . . . . . . . . . 163

REFERENCES • • • • • • • • • . • • • • • . • 169 APPENDIX A • . . . . . . . . . . . . . . . . . . 174 
LTST OF TABLFS

Table Paqe

4.1 Power Spectral Proqram Parameters . . . . . . 55

4.2 Cospectral Proqram Parameters . . . . . . . . 56

4.3 Variability of spectral fstimates . . . . . . . 57

5.1 Test Conditions . . . . . . . . . . 75

7.1 Evaluation of Universal Constant "a" . . . . . 120

7.2 Reynolds' Stresses . . . . . . . . . . . 121 


\section{LIST OF FIGURES}

Figure

Paqe

2.1 Wind Component Meter Turbulence Sensing Probe . . 20

2.2 Original wind speed Calibration Curve . . . . . 21

2.3 Wind Speed Calibration after Instrument Revision 22

2.4 Calibration Wind Tunnel . . . . . . . . . 23

2.5 Vertical Angle Calibration Curve . . . . . . . 24

2.6 Dependence of Vertical Angle Calibration on wind Speed . . . . . . . . . . . . . 25

2.7 Vertical Anqle Response Test Arrangement . . . . 26

2.8 Vertical Angle Response Records . . . . . . . 27

2.9 Speed Response Test Arrangement . . . . . . . . 28

2.10 Speed Response Test Records . . . . . . . . . 29

2.11 Horizontal Angle Response Test Arrangement . . 30

2.12 Horizontal Angle Response Test Records . . . . . 31

2.13 Basic Analog Computer Components and symbols . . 32

4.1 Coordinate Transformation Proqram . . . . . . 58

4.2 Function Generator Settings for Speed Calibration 60

4.3 Function Generator Settings for Vertical Angle Calibration at $5.0 \mathrm{mps}$. . . . . . . 61

4.4 Function Generator Settings for Vertical Angle Correction ............... . . 62

4.5 Coordinate Transformation Monitored Results . . . 63

4.6 Reynolds Stress Program . . . . . . . . . . 64 
Fiqure

Page

4.7 Reynolds Stress Monitored Results . . . . . . . 65

4.8 Power Spectral Filter Proqram . . . . . . . . 66

4.9 Power Spectral Monitored Results . . . . . . . 67

4.10 Cospectral Filter Proqram . . . . . . . . . 68

4.11 Cospectral Monitored Results . . . . . . . 69

4.12 Heterodyning Program . . . . . . . . . . 70

4.13 Heterodyning Monitored Results . . . . . . . . 71

7.1 Longitudinal wind Component spectra - Neutral Tests . . . . . . . . . . . . . . 122

7.2 Longitudinal wind Component Spectra - Unstable Tests... . . . . . . . . . . . . . 123

7.3 Longitudinal wind Component Spectra - Stable Tests . . . . . . . . . . . . . . 124

7.4 Longitudinal Wind Component Spectra - Tests Above 12 Meters . . . . . . . . . . . . 125

7.5 Longitudinal wind Component Spectra - Neutral Tests . . . . . . . . . . . . . . . 126

7.6 Longitudinal wind Component Spectra - Unstable Tests... . . . . . . . . . . . . 127

7. 7 Longitudinal wind Component Spectra - Stable Tests . . . . . . . . . . . . . . . 128

7.8 Longitudinal Wind Component Spectra - Tests Above 12 Meters . . . . . . . . . . . . 129

7.9 Vertical wind Component Spectra - Neutral Tests - 130

7.10 Vertical Wind Component Spectra - Unstable Tests 131 
Figure

Paqe

7.11 Vertical wind Component Spectra - Stable Tests . 132

7.12 Vertical wind Component Spectra - Tests Above

12 Meters . . . . . . . . . . . . . 133

7.13 Vertical wind Component Spectral - Neutral Tests 134

7.14 Vertical wind Component Spectral - Unstable Tests 135

7.15 Vertical wind Component Spectral - Stable Tests . 136

7.16 Vertical wind Component Spectral - Tests Above

12 Meters . . . . . . . . . . . . 137

7.17 Lateral wind Component Spectra - Neutral Tests - 138

7.18 Lateral wind Component Spectra - Unstable Tests . 139

7.19 Lateral wind Component Spectra - Stable Tests . . 140

7.20 Lateral Wind Component Spectra - Tests Above 12 Meters ................. . 141

7.21 Lateral wind Component Spectra - Neutral Tests . 142

7.22 Lateral wind Component Spectra - Unstable Tests . 143

7.23 Lateral wind Component Spectra - Stable Tests . . 144

7.24 Lateral wind Component Spectra - Tests Above 12 Meters . . . . . . . . . . . . 145

7.25 Cospectra Between Longitudinal and Vertical Velocity - Neutral Tests . . . . . . . 146

7.26 Cospectra Between Longitudinal and Vertical Velocity - Unstable Tests . . . . . . . . 147

7.27 Cospectra Between Longitudinal and Vertical Velocity - Stable Tests . . . . . . . . . 148

7.28 Cospectra Between Longitudinal and Vertical

Velocity - Tests Above 12 Meters . . . . . 149 
Figure

Page

7.29 Cospectra Between Lateral and Vertical Velocity

- Neutral Tests............. . . 150

7.30 Cospectra Between Lateral and Vertical Velocity

- Unstable Tests . . . . . . . . . . 151

7.31 Cospectra Between Lateral and Vertical Velocity

- Stable Tests . . . . . . . . . . . 152

7.32 Cospectra Between Lateral and Vertical Velocity

- Tests Above 12 Meters . . . . . . . . 153

7.33 Cospectra Between Longitudinal and Lateral

Velocity - Neutral Tests . . . . . . . . 154

7.34 Cospectra Between Longitudinal and Lateral

Velocity - Unstable Tests . . . . . . . 155

7.35 Cospectra Between Longitudinal and Lateral

Velocity - Stable Tests . . . . . . . . 156

7.36 Cospectra Between Longitduinal and Lateral

Velocity - Test Above 12 Meters . . . . . 157

7.37 Cospectra for Test 13-1 . . . . . . . . . 158

7.38 Average Longitudinal wind Component Spectrum • . 159

7.39 Average Vertical Wind Component Spectrum . . . 160

7.40 Average Lateral wind Component Spectrum . • . . 161

7.41 Example of Variability in Cospectral Calculation 162 
. 
EXPERIMENTAL INVESTIGATION OF THE TURBULFNCE STRUCTURE IN THE LOWER ATMOSPHERF

\section{INTRODUCTION}

In recent years, since fast response meteorological instruments have been developed capable of measuring rapid fluctuations in the atmospheric variables, data have been accumulating on the statistical properties of atmospheric turbulence. Turbulent intensities of the variables, turbulent fluxes in the atmosphere and spectral distributions of these quantities have been measured with increased regularity and dependability. However, much of the effort remains directed toward developing the measurement, data collection and analysis systems, and extensive experimental programs for defining atmospheric turbulence structure are still forthcoming. Individual investigations have been often limited in several aspects including the number of components measured, the height of measurement, and the frequency range studied (Iimited at low frequencies by the length of record and at high frequencies by instrument response or sampling rate). Furthermore, the variations of the statistical characteristics of the measured quantities with varying average states of the atmosphere have not been fully explored. Consequently, much experimental work remains to be done to demonstrate adequately the structure of turbulence in the atmospheric 
boundary layer. Theoretical work ${ }^{1}$ has been successful in describing turbulence primarily for isotropic conditions and direct application to the atmosphere has been limited generally to the high wave number range where local isotropy and its consequences in the atmosphere have met with some success although the extent of these experiments generally covered only a few of the aspects of the locally isotropic turbulence. The considerable verification of Kolmoqoroff's $-5 / 3$ law has generally lacked simultaneous demonstration of the dependence on $\varepsilon^{2 / 3}$ and the reduction of the cospectra to zero as required by local isotropy.

More recent theoretical work, the "similarity theory" of Monin and oboukhov 28 and clarifying discussions by Panofsky 29 and Ellison ${ }^{10}$ have contributed insight into the structure of the anisotropic, energy producing range of atmospheric turbulence and its relation to the distributions of the mean variables. An extersion of this dimensional line of reasoning, discussed by Gurvichl5 has led to some organization of measurements of turbulence parameters and spectral distributions anticipating similarity relationships in turbulence data. However, for the most part, measurement demonstrating theoretical relationships and organization of data defining the structure of atmospheric turbulence is far from completed. The difficulty in obtaining adequate turbulence data did not end with the development of fast response instrumentation for measuring fluctuations of atmospheric variables. 
The collecting, samplinq, and analysis of such data has introduced difficulties that continue to impede proqress in the experimental study of turbulence structure. Digital analysis of turbulence data requires sampling at a rate on the order of ten times the highest frequency of interest. Digital sampling technology has advanced rapidly, so that automatic analog to digital conversion can be performed simultaneously with the measurement of variables at a rate adequate to investigate many turbulence problems of interest. However, advances have likewise been made in instrumentation, allowing other turbulence studies at fluctuation frequencies presently beyond practical digital sampling capabilities. For example, on the one hand, the turbulent flux of momentum as well as heat and moisture in the atmosphere can be studied with digital analysis techniques; little difficulty arises in sampling the necessary turbulent fluctuation measurements at an adequate rate. On the other hand, fluctuation measurements in the dissipation range of eddy sizes would be extremely difficult to sample and analyze with digital equipment.

Although digital analysis methods have been used for most atmospheric turbulence studies a number of investigations have utilized specialized analoq devices for analyzing continuous real-time and tape-recorded measurements. These devices are often limited in their accuracy and the extent of analysis that can be carried out with them. As instrument accuracy improves and as more varied and complex analysis 
of turbulence data is desired, such devices will no longer be adequate for meeting the analysis needs.

The general purpose analog computer, highly accurate, responsive to any range of frequencies of interest in the atmosphere, and easily programmed to handle a wide variety of problems, is well suited to the detailed analysis of continuous turbulence data. The application of this versatile tool to analysis of turbulence data has not been extensively developed.

The availability of a general purpose analog computer in Battelle-Northwest Laboratories at Hanford, Washington (an AEC installation and site of this study) has made it possible to investiqate this means of analyzing turbulence data.

The purpose of this study, then, is to organize the turbulence statistics such as total variances and covariances of the three wind components in addition to spectral and cospectral distributions, over a wide range of frequencies, according to heights, wind speeds, and stabilities in order to test the Monin-oboukhov similarity theory as it applies to them and to determine the forms of any universal functions involved. To accomplish this a sizeable quantity of turbulence data for all three components will be collected and the analog computer will be adapted to analyze the data for the above-mentioned statistics. 


\section{EQUIPMFNT}

\section{Turbulence Sensing Fquipment}

The turbulence data presented here were collected at Hanford with a fast response sensor, termed the wind component meter. The sensor signals were recorded on a magnetic tape recorder and later analyzed on an analoq computer.

The wind component meter, originally designed by J. J. Fuquay of Battelle Northwest Laboratories at Hanford, is similar in some respects to earlier sensors described by $G$. C. Gill $^{14}$ and H. F. Cramer $^{7}$, utilizing heated thermocouple wires. M. Miyake 24 has developed an improved version of the wind component meter with heated thermocouple sensors similar to the Fuquay model but incorporating more complex electronic circuitry. Further reference will be made to Miyake's instrument following the discussion on the response of the wind component meter.

The instrument shown in Fiaure 2.1 senses the three components of the wind at a given single point and produces continuous voltage signals related to the three spherical wind components. The signal for the speed, V, is a fluctuating dc signal across a bridge circuit, two legs of which are chromel-alumel thermocouple wires heated by $610 \mathrm{cps}$ alternating current impressed across the thermocouple wires at a constant, controlled voltage. The thermocouples are suspended in and cooled by the air stream while the reference 
junctions formed at the mounting studs are maintained at ambient temperature. Another thermocouple, unheated, removes the fluctuations in the signal due to ambient temperature variations. The emf produced by this thermocouple arrangement is filtered to remove residual heating current and extraneous noise.

The signal for the elevation or vertical angle, $\phi$, is also derived from heated thermocouple wires. There are two of these inclined at $45^{\circ}$ angles to the horizontal and $90^{\circ}$ to each other, forming a "V" in the vertical plane. The thermocouple output is zero for horizontal winds and varies positively and negatively for downward and upward gusts, respectively. This signal is also filtered to remove noise. The sensor head is continuously driven to remain oriented in the direction of the horizontal wind, keeping the wind normal to the speed sensing thermocouple wires at all times and keeping the wind in the plane of the elevation angle sensing " $V$ "-shaped thermocouple arrangement. This is accomplished by a second " $V$ "-shaped arrangement of platinum wires in the horizontal plane which provides an error signal to a servo amplifier and motor. This continually drives the probe to the null position, orienting the head into the instantaneous wind. A potentiometer is also connected to the shaft of the servo motor so a signal proportional to the horizontal angle, $\theta$, is produced to be amplified and recorded with the wind speed and vertical angle. 
Fiqure 2.1 shows the sensing probe, containing the thermocouple head with associated bridge networks and the servo motor. A $350 \mathrm{ft}$ cable connects the sensing head with the electronics, which includes the heating current oscillator. the servo amplifier, the signal filters, and Flectro-Instruments differential dc amplifiers to boost the signals to recording level. Wiring diagrams for the wind component meter are given in an unpublished report by Ratcliffe and Sheen $^{40}$.

Calibration of the wind component meter was accomplished first by comparing the speed signal with the standard cup anemometer in the wind tunnel of the Department of Atmospheric Sciences, the University of Washington. The calibration curve gave the nonlinear relation between the instrument output and the true wind speed shown in Figure 2.2. Later, when the instrument was improved, a change in the probe heating current changed the wind speed signal from the previous signal level by a constant factor for all wind speeds. The new calibration shown in Figure 2.3 was made by comparing wind component meter signals with corresponding wind data from new Beckman and whitley anemometers on the Hanford Portable Mast. Speed calibration at very low velocities was also accomplished in a small wind tunnel at Hanford made from a 6-inch diameter plastic tube. Turbulence pulses were generated and their transport times over a known tunnel distance were measured. These points are shown as circles 
in Figure 2.3.

Calibration of the elevation angle signal was performed in the same small wind tunnel. The thermocouple head, which can be removed with an extension cable from the shaft of the wind component meter, was inserted into the tunnel and inclined at various angles to the air stream. The calibration tunnel is shown with the wind component meter probe in Figure 2.4. The resulting calibration curve provided a nonlinear relation between the signal and the elevation angle. This calibration is shown in Figure 2.5. It is noted that the elevation ande calibration is a function of wind speed. This dependence, determined from repeated calibrations at various tunnel speeds, is shown in Figure 2.6 .

The horizontal angle signal is linearly related to the true angle, and the constant of proportionality is established by the known voltage across the potentiometer providing the horizontal angle signal.

Tests of the response times of the instrument were also made in the 6-inch calibration tunnel. The vertical angle response was determined by oscillating the thermocouple head, removed from the wind component meter with an extension cable and rotated in the tunnel about an axis concentric with the thermocouple junctions for the vertical angle measurement. The head was rotated through a $26^{\circ}$ arc in the vertical plane at a number of speeds with a variable 
speed, motor driven mechanism, simulating vertical angle fluctuations of various frequencies. Fiqure 2.7 shows the arrangement for this test. The vertical angle signal was recorded on a Honeywell Visicorder Oscillograph which utilizes a mirror galvanometer projecting a light beam onto light sensitive paper so that signal oscillation frequencies much larger than those of concern here can be reproduced without amplitude or phase distortion. The record for the vertical angle response is shown in Figure 2.8 , demonstrating that the instrument output is not reduced in amplitude for frequencies up to about 4 cps; close to 5 cps a slight reduction is suggested. Also comparing the recotd with references pulses generated at the same point for each oscillation, no phase shift can be detected for the vertical angle signal over the range of frequencies tested. The records of Figure 2.8 were for wind speeds of $2.0 \mathrm{mps}$ and $7.0 \mathrm{mps}$.

The time response for the speed signal was tested in the same tunnel by sweeping the thermocouple head through an $8.1^{\circ}$ arc about an axis outside the tunnel at a radius of 23-1/4 inch so that the sensing wires were oscillated essentially in the logitudinal direction. The test set-up is shown in Figure 2.9. This motion superimposed speed fluctuations on the steady tunnel flow, the magnitude of which was determined by the oscillation frequency. The peak values of the speed fluctuations, calculated for various oscillation frequencies up to almost $4 \mathrm{cps}$ are compared in Figure 
2.10 with the signals produced by the instrument.

For $2.0 \mathrm{mps}$ and $7.0 \mathrm{mps}$ tunnel speeds, the measured peak values of the speed oscillations compare well with the calculated siqnal levels at all frequencies tested. The speed sensing capability at the wind component meter demonstrates no significant loss in response to about $4 \mathrm{cps}$. The reference pulses again fail to reveal any appreciable phase shift.

The horizontal andle response was tested by placing the wind component meter on a rotating platform with the thermocouple head, connected to the shaft, inserted into the wind tunnel from beneath as shown in Fiqure 2.11. The platform was oscillated through a $43^{\circ}$ arc in the horizontal plane at frequencies up to $2 \mathrm{cps}$. Thus the head moved relative to the body of the wind component meter, being driven continuously by the servo system into the air flow along the axis of the tunnel. The horizontal angle signal, produced by the rotation of the shaft, was recorded on the visicorder where deviations from a constant peak amplitude, as well as the observed movement of the thermocouple head away from the axial direction for high frequency oscillations, would indicate a reduced response. However, for the range of frequencies tested, limited at about $2 \mathrm{cDs}$ by the mechanical capabilities of the motor-driven rotating platform, Fiqure 2.12 shows no reduction of the peak amplitude. In fact, at a frequency of 2 cps an overshoot was detected, increasing the peak amplitude 
by about 10\%, as well as distorting the wave form and causing a small apparent phase shift. The platform was rotated through a $14^{\circ}$ arc by hand to simulate higher frequency fluctuations and the thermocouple head was observed to remain oriented in the direction of the tunnel axis and no significant signal reduction was noticed at least to frequencies near 4 cps. The smaller angle of rotation used in this case is more consistent with magnitudes to be observed in wind fluctuations at high frequencies. The results indicate that errors in excess of those introduced in the analysis of the data are not expected for the horizontal angle below 4 cps.

In the tests described above, complete response curves of the instrument were not determined but it was found that fluctuation measurements of all three components could be used uncorrected up to 4 cps and possibly higher with only minor errors resulting.

Though the frequency response of the wind component meter as described above is somewhat faster than Miyake's improved model which begins to show limitations in its response at $1 \mathrm{cps}$, the latter has the advantage of good sensitivity at all wind speeds whereas the Hanford wind component meter loses sensitivity at high wind speeds.

Recording Equipment

A seven-channel Ampex FR-1100 magnetic tape recorder with FM electronics was used to record the turbulence signals. 
Tape transport speeds of $3-3 / 4,7-1 / 2,15$, and 30 in. per sec (ips) areselectable for recording or playback. once recorded, the data from a given series of tests were stored until analysis on the analog computer could be scheduled.

A seven-channel Ampex FR-1300 magnetic tape recorder/ reproducer with FM electronics was used for reproducing the data at the time of analysis. Tape transport speeds of $1-7 / 8,3-3 / 4,7-1 / 2,15,30$ and 60 in. per sec are selectable. Both tape recorders record and reproduce over a nominal \pm 1.0 volt rms range. The use of the tape recorders not only allowed the turbulence signals to be stored until a convenient time for analysis but recording and playing back at different speeds allowed the data to be compressed, shifting the fluctuation frequencies of interest into a higher range easily handled by the electronic analog computer and greatly reducing the analysis time.

Both tape recorders are highly accurate, capable of reproducing a recorded signal with little distortion but their limitations as outlined in the manufacturer's specifications must be considered for an assessment of the errors introduced. The tape transport introduces noise due to mechanical flutter which does not exceed an amplitude of $1.5 \%$ of the full scale amplitude for frequencies below 312 cps at the lowest transport speed and the error is considerably decreased for faster speeds. Consequently, for atmospheric fluctuations of interest, this error is minimal. A 
start time of less than 8 sec before the transport reaches stable tape motion is required at the fastest transport speed. Care was taken to assure the motion was stable in order not to introduce initial low frequency oscillations in the data.

Differences in the relative positions, from one channel to another, of the recording and reproducing gaps on the tape heads introduce interchannel time displacement errors. If large, such increments could introduce serious errors when signals from different channels entered into the same computation in coordinate transformations and calculations of covariances and cospectral estimates. However, the time displacement error does not exceed $5 \mathrm{micro-sec}$ at 60 in. per sec tape speed. Consequently, even though the analysis time base was reduced by as much as $1 / 256$ of the oriqinal measurement time, a time displacement error between the original signals on the order of 1 milli-sec was not exceeded and is of no concern in the present study. The FM electronics for the FR-1100 have a flat frequency response from 0 to $625 \mathrm{cps}$ at $3-3 / 4$ ips, eásily covering the range of atmospheric fluctuations of concern during the initial recording. The FR-1300 electronics have a flat response from 0 to 625 cps at $1-7 / 8$ ips, assuring no alteration of the relative amplitude of the various frequency components over the range of fluctuations being studied during the analysis. The signal to noise ratio for the 
electronics of the FR-1300 recorder does not fall below 40 $\mathrm{db}$ and for the FR-1100, below $35 \mathrm{db}$ so that a noise signal will not exceed 1 to 28 of the l-volt rms full-scale signal at the slowest tape speeds and will be improved at faster tape speeds. The total harmonic distortion does not exceed 28 for either recorder and the linearity is $\pm 1 \%$ of full scale. Thus it can be expected that errors on the order of 1 to 28 will generally be introduced into the signals by the tape recorders. For the higher frequency and lower amplitude signals, slightly larger errors can be expected.

Analysis Equipment

The analysis of the tape recorder turbulence data was carried out on an Ease 1132 general purpose electronic analod computer. Since the programs used on this computer for the analysis will be given later, involving the generally accepted symbols for the various computer components, the symbols are identified here for the components used and their functions are described briefly. For a more complex discussion see Johnson 16 and Korn and Korn ${ }^{18}$.

An electronic analog computer can analyze a continuous voltage varying as a function of time. Such a siqnal can be treated by circuits which sum, multiply, differentiate, integrate, and filter the voltage. These circuits can be combined to give electrical analogs of the mathematical procedures of interest. 
Basic to the electronic analog computer is the high gain dc amplifier with resistive and capacitive feedback networks called the operational amplifier. Along with potentiometers, diodes and other electronic components, the operations of multiplication, division, function generation, and transformation and rotation of coordinates can be included. Besides the application of the computer to data analysis, as planned here, it is more generally used for solving differential equations and simulating complex systems. Figure 2.13 shows the symbols used to indicate each of the electronic units performing the functions discussed here. Amplifier circuits or block diagrams more completely describing their operation are also included.

The summing of a number of input signals is performed by impressing each of them across one of the input resistances of the high gain dc amplifier with resistive feedback shown in Figure 2.13a. The negative of the output voltage is equal to the sum of the input voltages each multiplied by a gain factor which is simply the ratio of the feedback resistance to the input resistance. Gains of 1 and 10 , which can be selected by changing the input resistances, are very precisely controlled with precision resistors, accurate to within 0.018 . The integrator shown in Figure $2.13 \mathrm{~b}$ utilizes the same type of high gain dc amplifier but with capacitive rather than resistive feedback. The output voltage is proportional to the integral of the input voltage where the proportionality 
constant, $\frac{-1}{\mathrm{RC}}$, can very accurately determine a selectable gain through use of precision input resistances and feedback capacitors. Besides the limits of precision determined by passive components, errors in integration are possible from a number of sources. However, these are generally less than 18 for computers with high quality components. For a thorough discussion of integrator errors see Korn and Korn ${ }^{18}$. The frequency response of high gain amplifiers, summers and integrators is flat to $20 \mathrm{kc}$, far more than adequate for the analysis of the present problem. The noise level for the active components is about $5 \mathrm{mv}$, peak to peak, so signal levels should be kept at least on the order of a volt. Multiplication by a constant can be performed in the adding or integrating circuits, as described earlier, by adjusting the input or feedback resistances or capitances. A constant multiplier qiving any desired fraction of the signal is also provided through preset potentiometers inserted in the analog program at any point. The symbol for this operation is shown in Figure 2.13c. Multiplyinq two variables together is accomplished with time division multipliers in the Ease 1132 computer, shown in Figure 2.13d, which averages a series of pulses the amplitudes of which are determined by one of the variables and the duration of which are determined by the other. The pulse rate is the limiting factor for determining the frequency response of the multiplier which is flat to 500 cycles. This is sufficient for the 
present study althouah for some problems it can present serious limitations. The noise level, about $15 \mathrm{mv}$ (peak to peak), can also be large compared to the signal, exceeding a level of 18 of the output siqnal if inputs fall below 10 volts. The most serious of the multiplier errors, however, is that introduced by drift of the output siqnal. Low frequency variations of about $20 \mathrm{mv}$ in the output are common. The seriousness of the drift error results from its low frequency character so that an inteqration of a multiplier output can accumulate a sizeable error from the multiplier drift, whereas the multiplier noise will contribute nothing to the integral.

A function generator can be used to approximate any single valued function of a variable dependent on time with a series of straight line segments so that the value of the function is given continuously from the function generator as the time dependent variable is applied to the input. They are particularly useful in representing non-analytic functions such as experimentally obtained curves. Function generators were used in this study to represent the instrument calibration curves. A function generator is comprised of a series of circuits like the one shown in Figure $2.13 \mathrm{e}$ the outputs of which are summed. Each circuit has a breakpoint and a slope potentiometer setting which determine short linear segments of the function approximated. The most serious errors resulting from the use of a function generator, rather than 
being introduced by the limitations of the electronics, are related to the shape of the functions and how accurately it can be approximated with straight line segments. In the present study, errors arising from generation of calibration curves in the computer programing should not exceed $5 \%$ and will average less.

Coordinate transformations are performed by resolvers which take as inputs a signal representing an angular measurement and one representing a radial measurement and qive as outputs the cartesian components. This is accomplished by combining the operations of multipliers and a function generator. From the anqular measurement input, sine and cosine functions are generated by the function generator. These and the radial measurements are inputs to the multipliers, the outputs of which are then the cartesian components, as shown in Fiqure 2.13f. The accuracy of the resolver is limited by that of the muitipliers, previously discussed, as well as by the function qenerator. According to the computer specifications in this case, the function generator in the resolver is accurate to $0.05 \%$.

Though other internally wired components can be included in analoq computers, those discussed above are most fundamental and are the only components pertinent to the analysis of the present problem. The manner in which these components are interconnected or programmed for the analysis will be described later. Also to be described later are 
externally wired filters, necessary components in the programs to be discussed. 


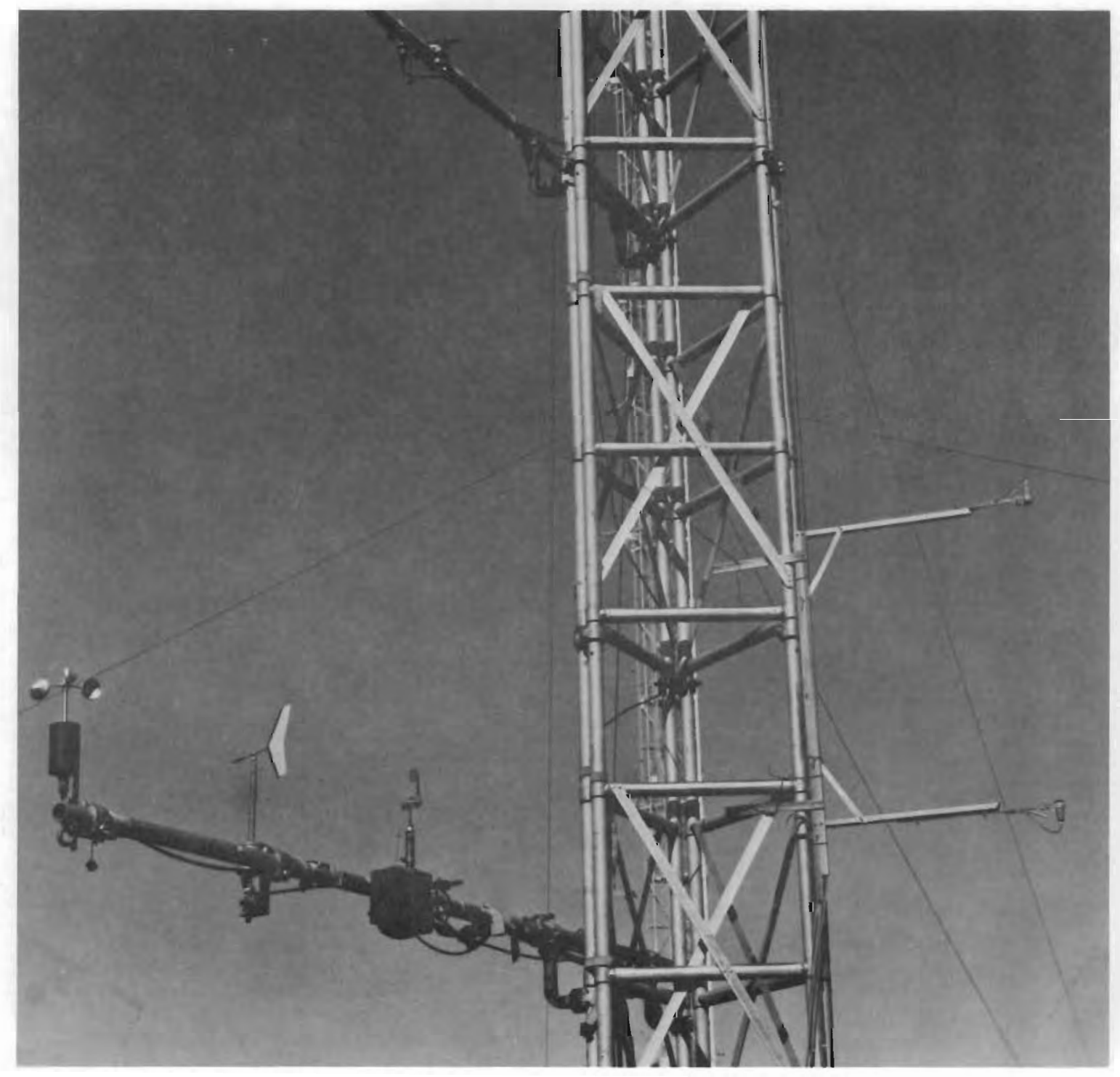

FIGURE 2.1 Wind Component Meter Turbulence Sensing Probe 


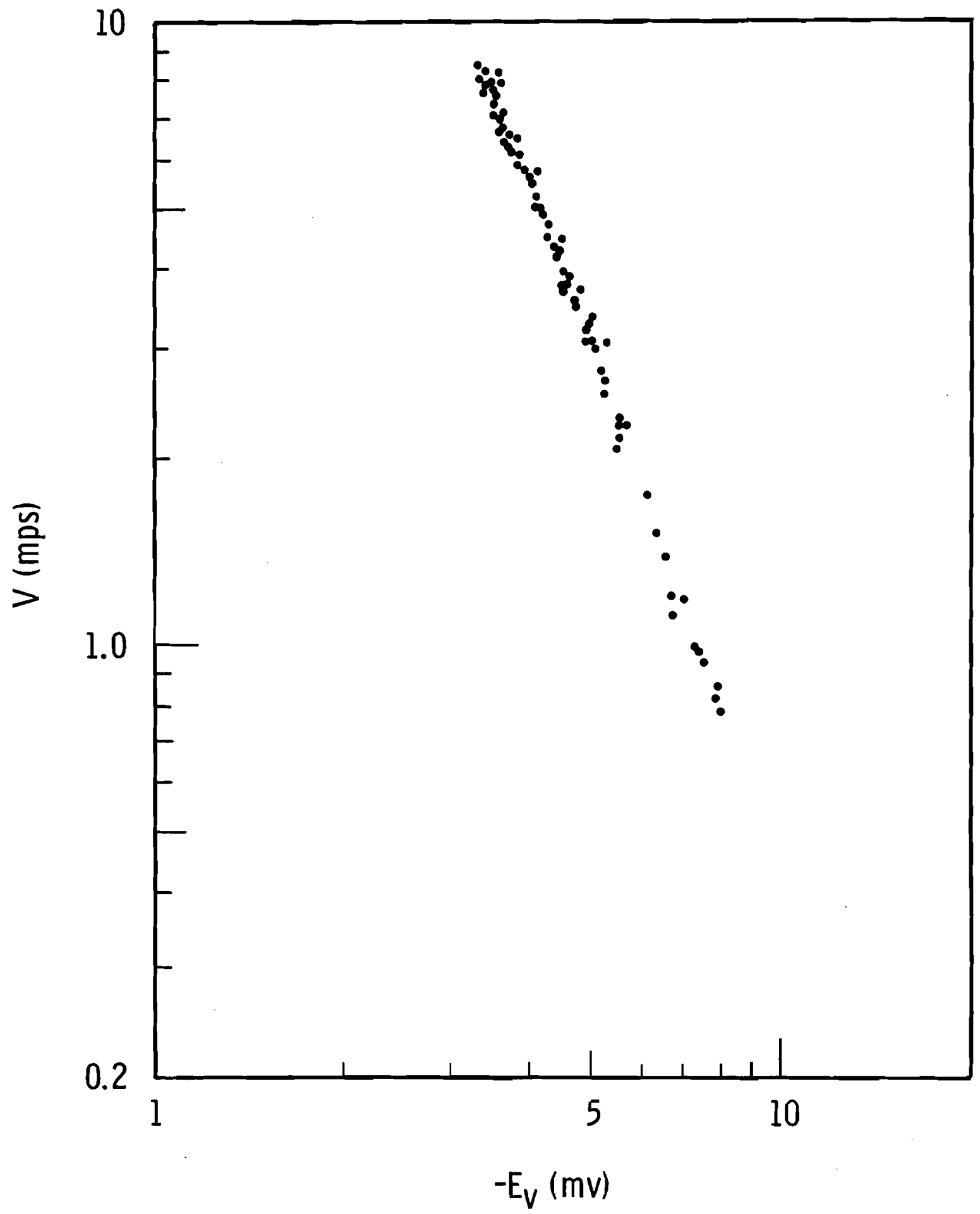

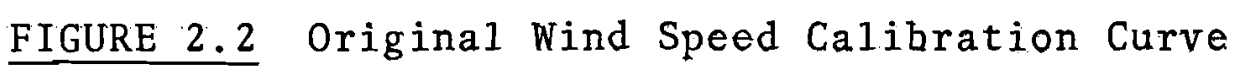




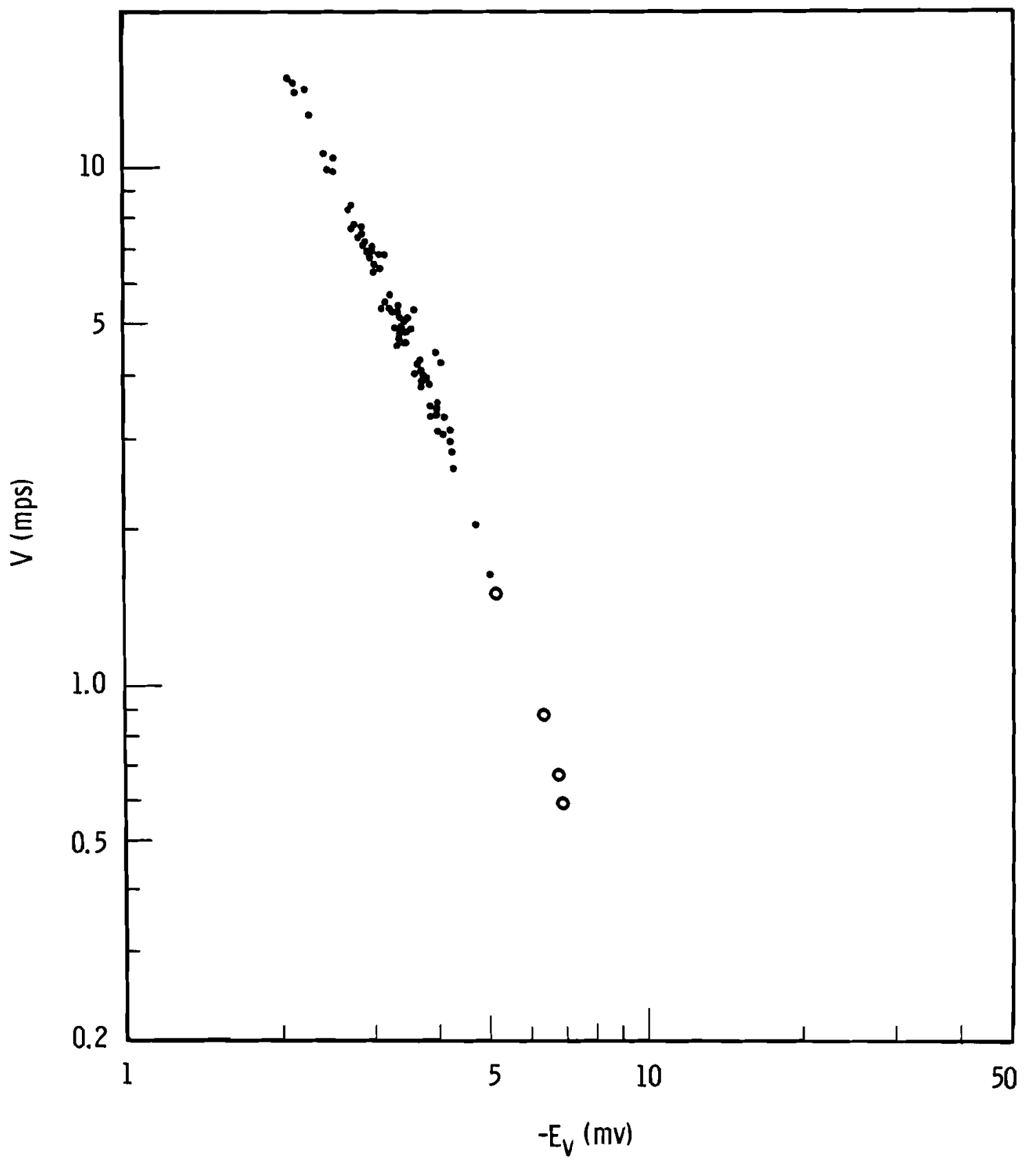

FIGURE 2.3 Wind Speed Calibration After Instrument Revision 


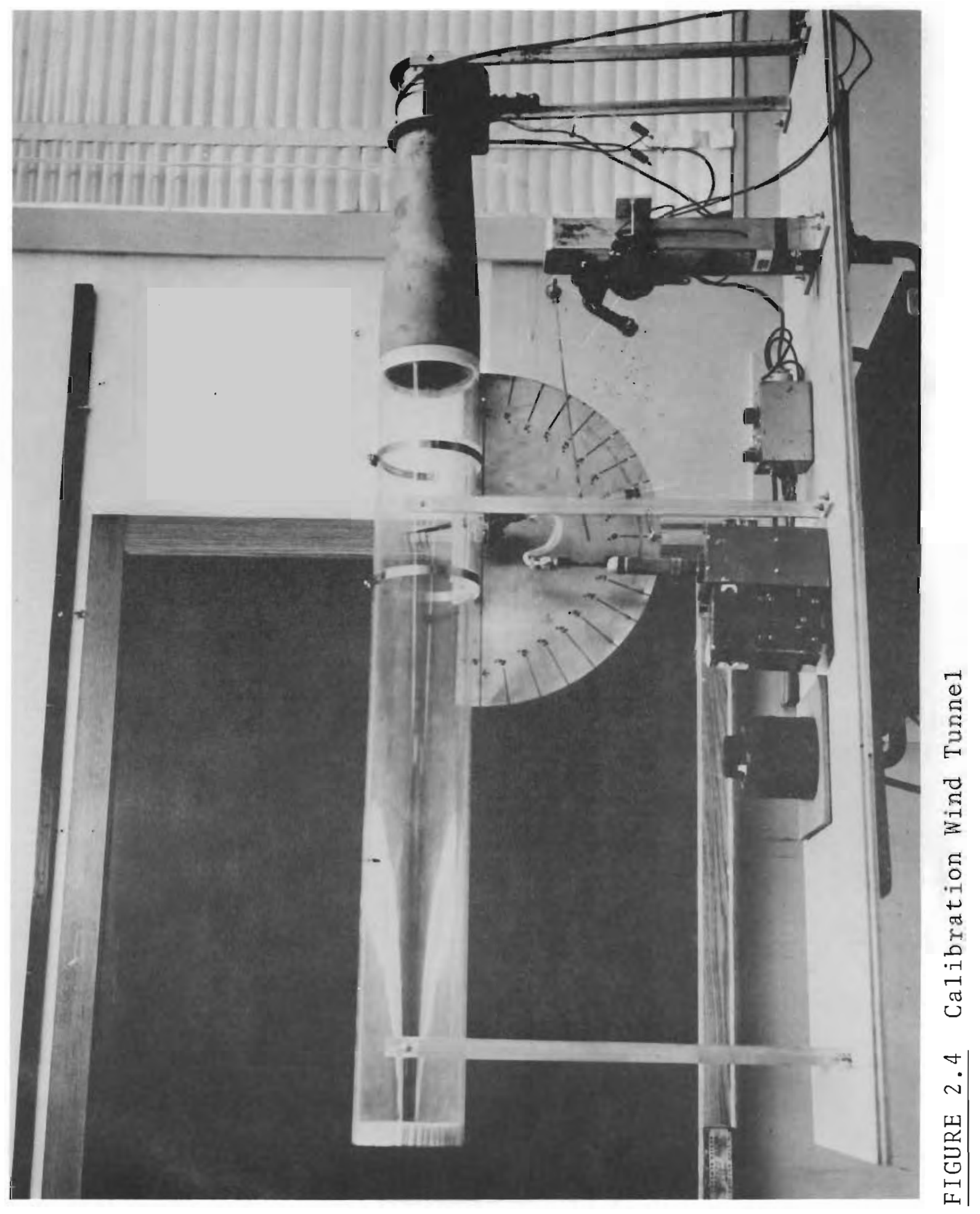




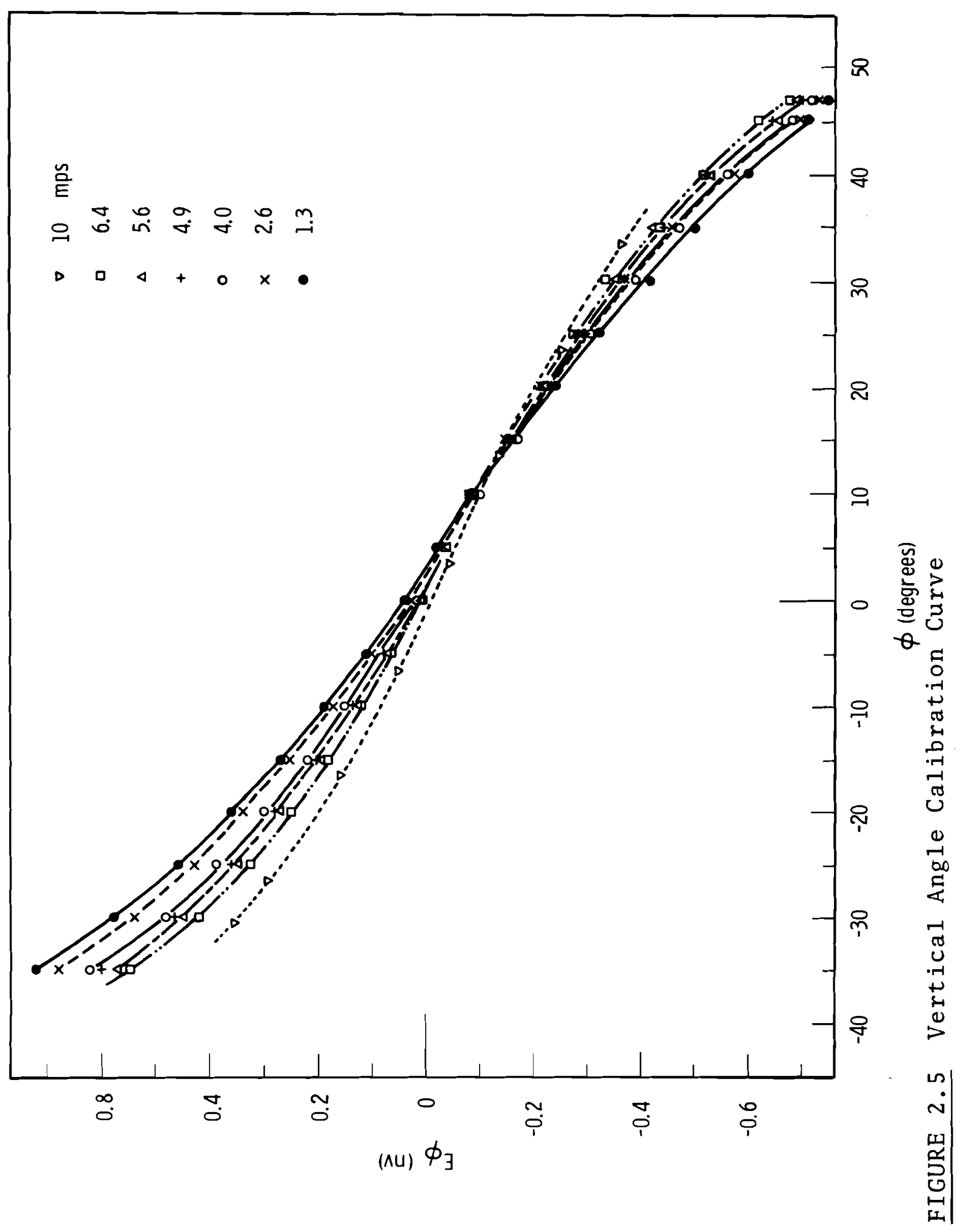




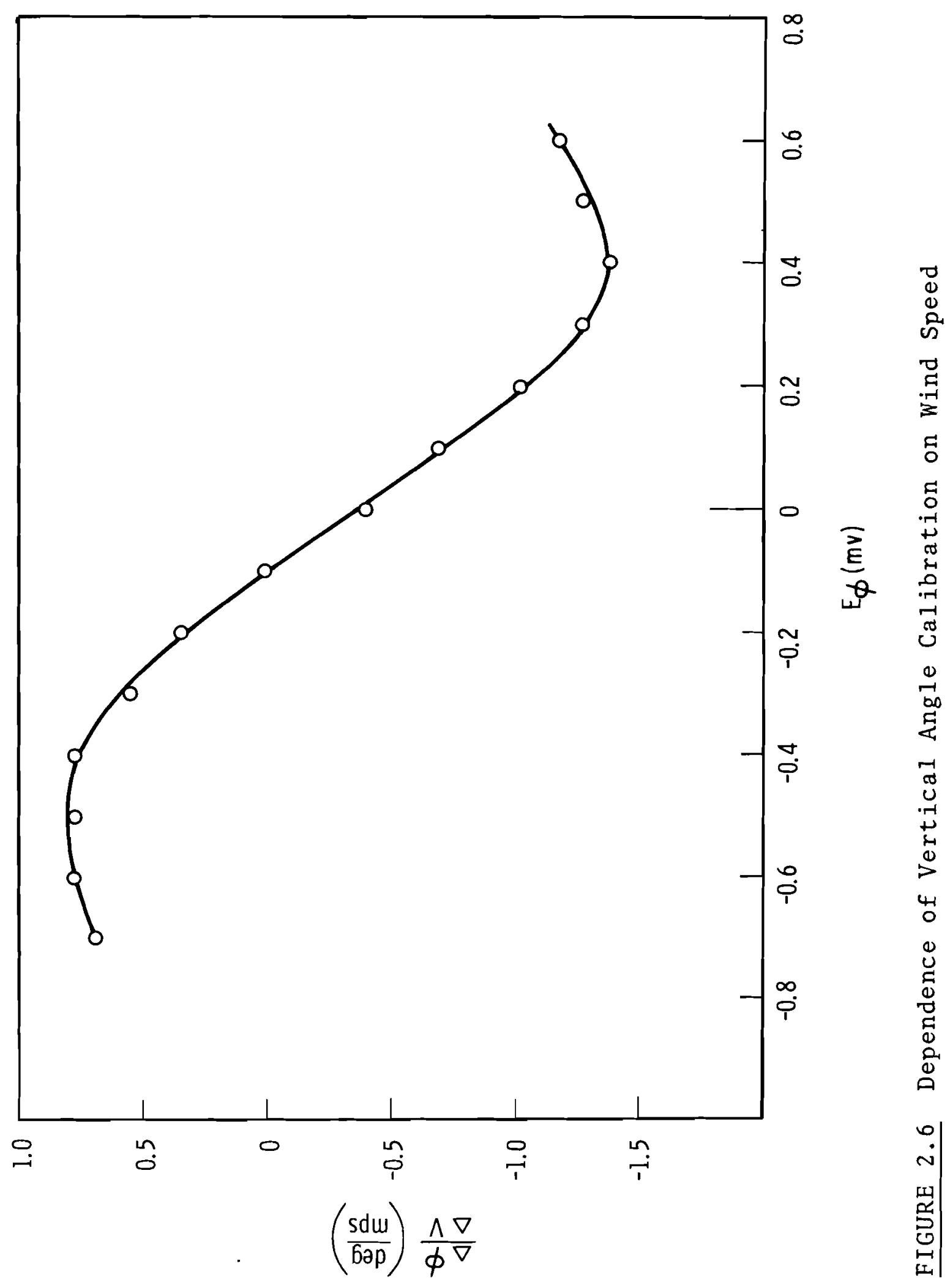




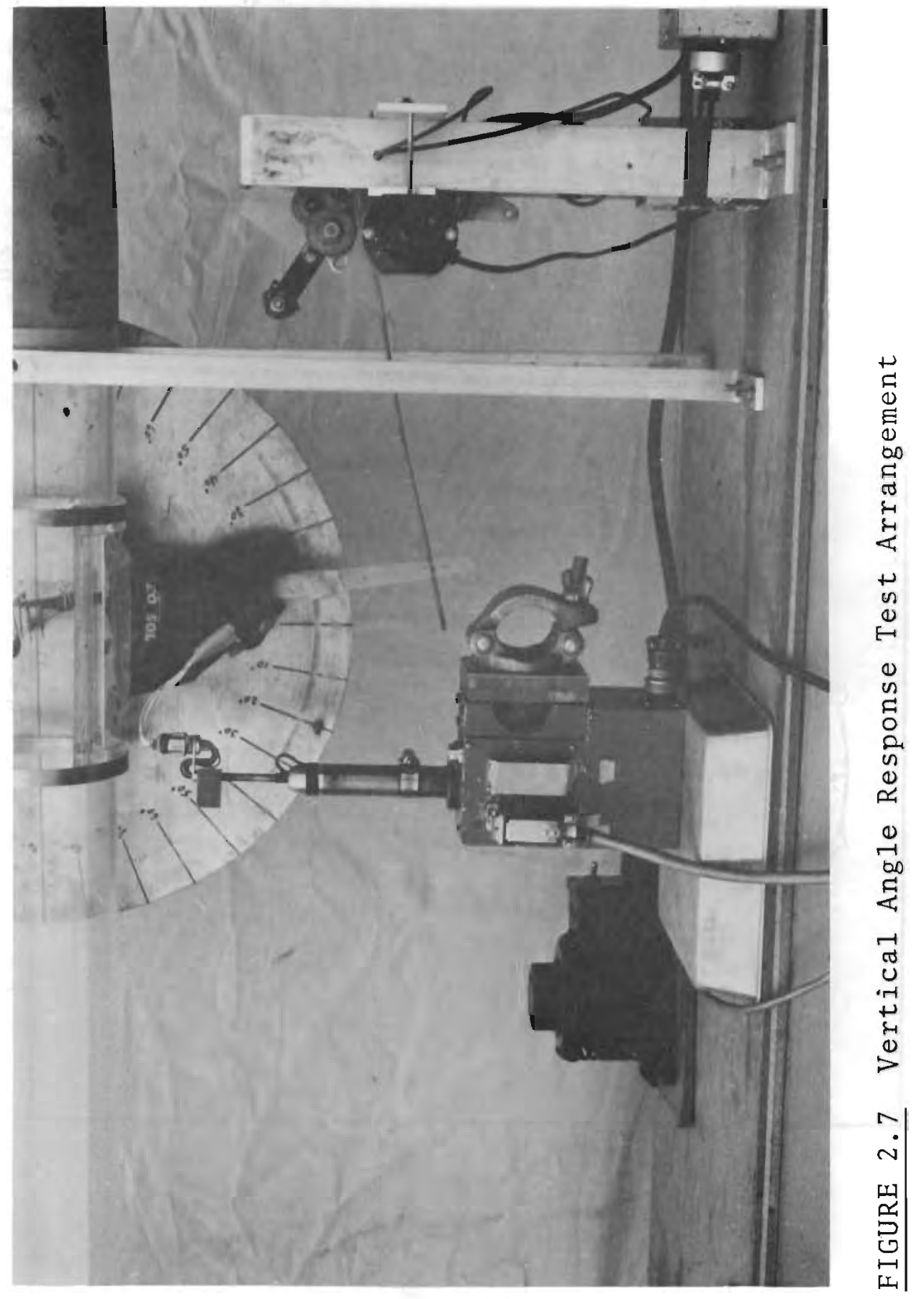


(spuоวәS) วய!1

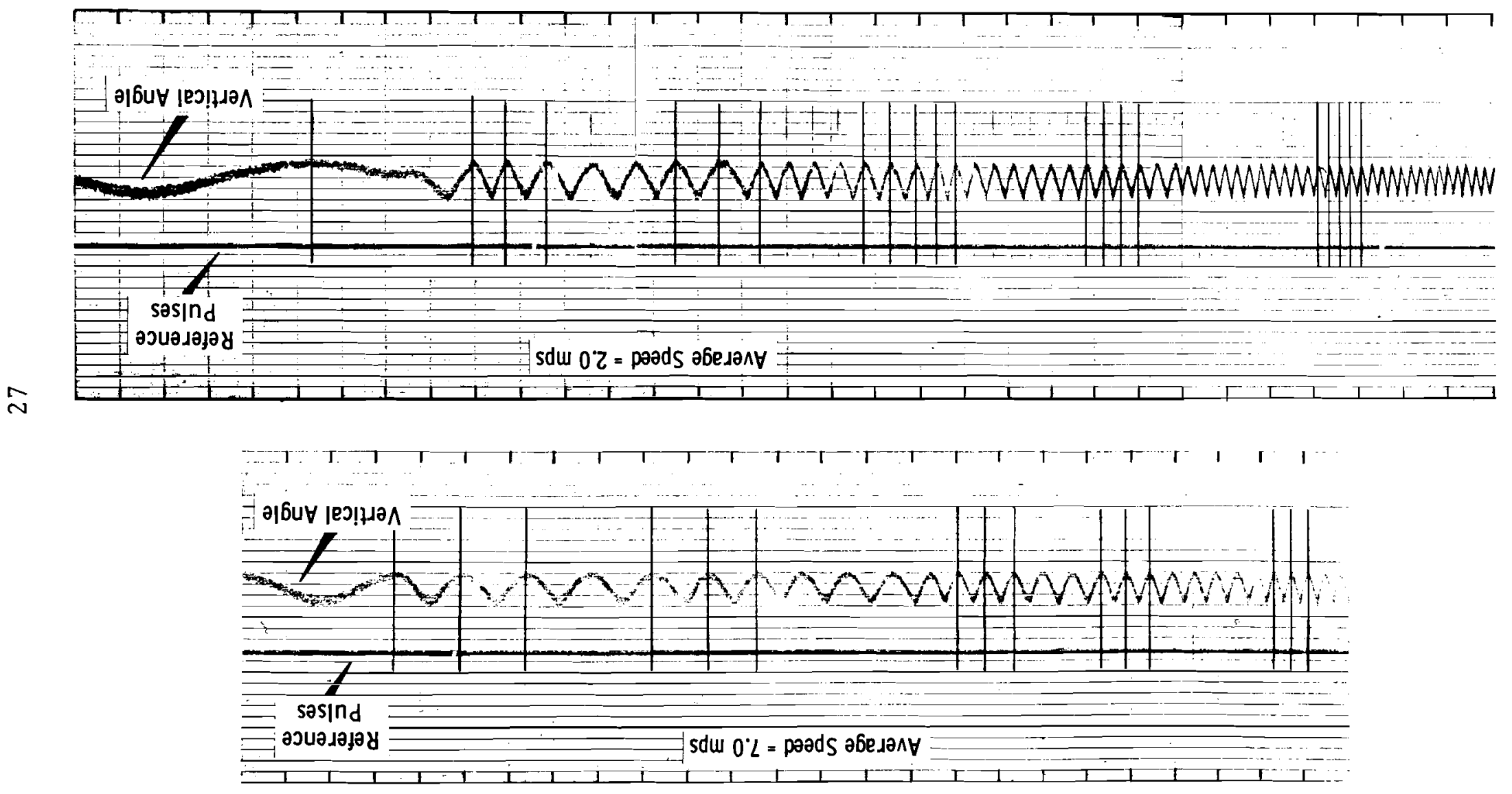




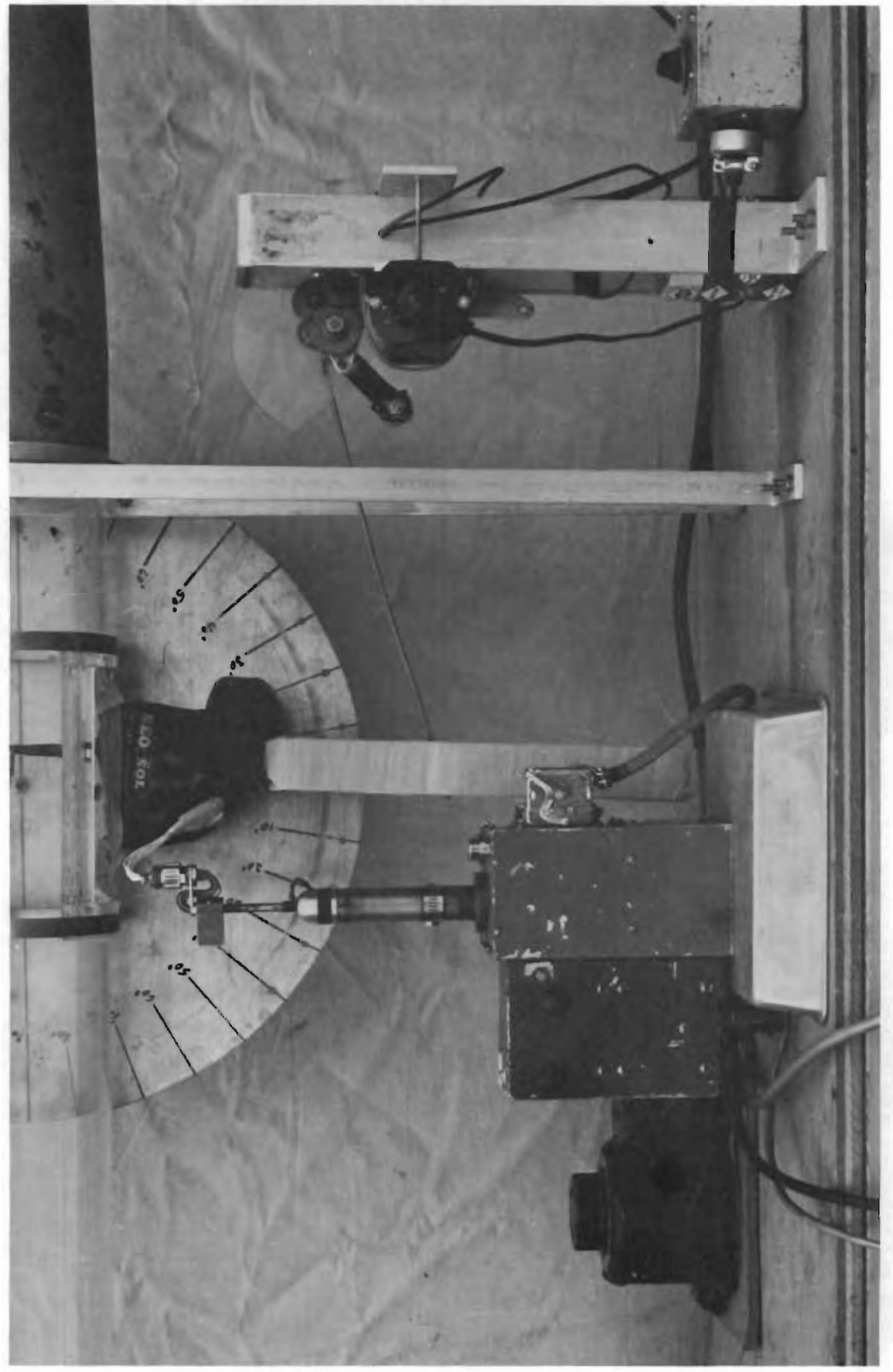

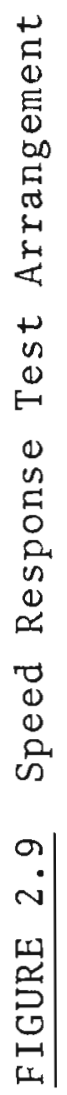


(SpU0JэS) วய!1

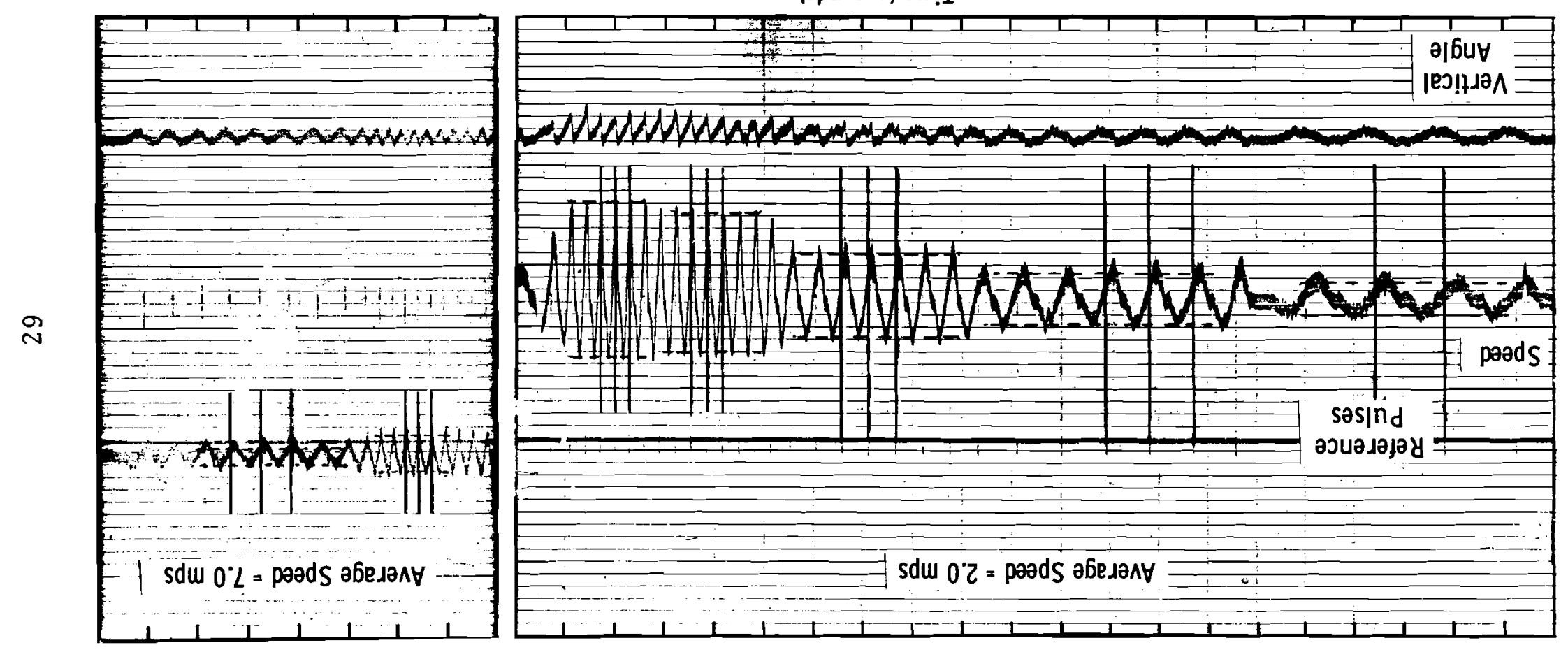




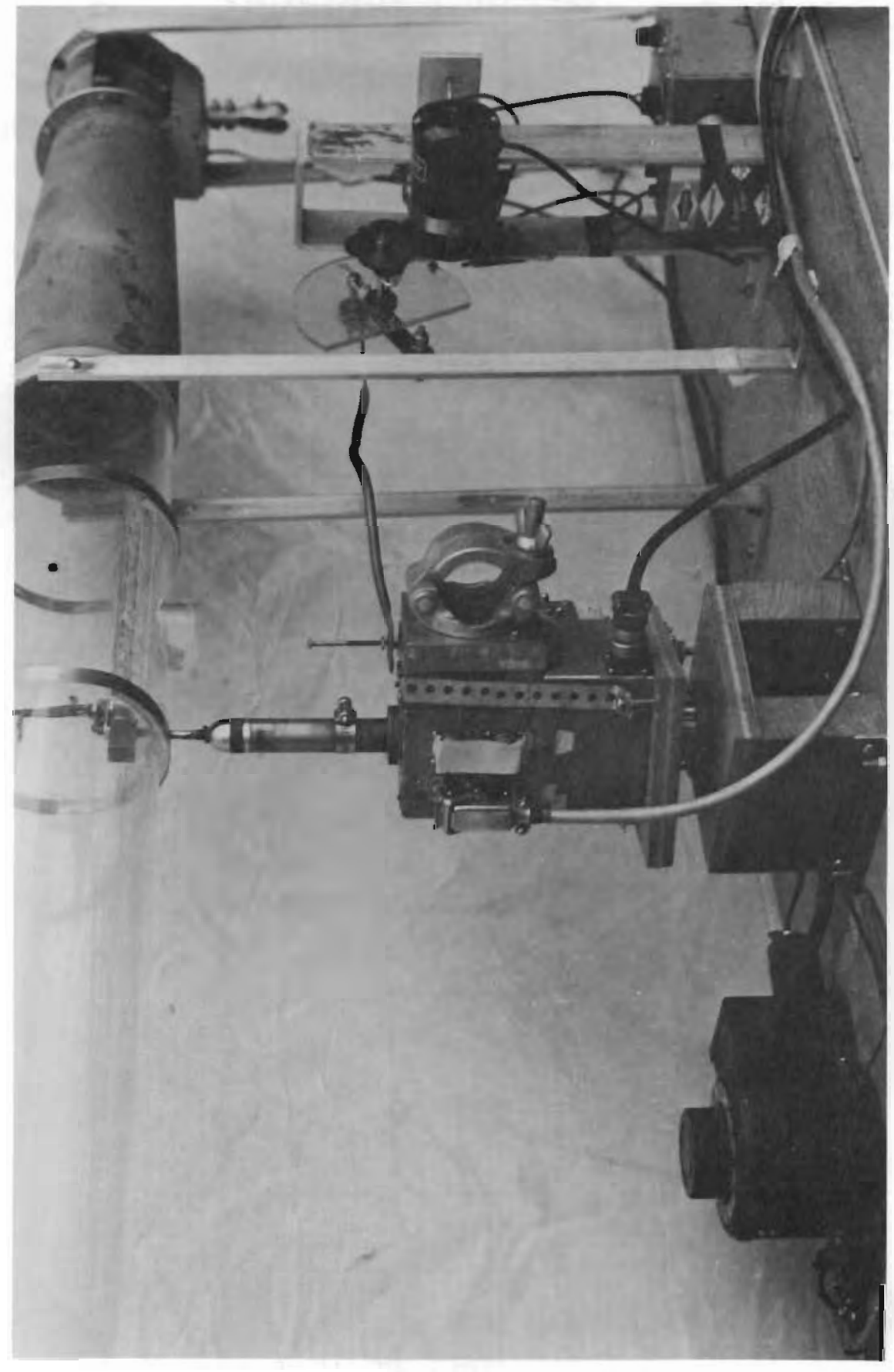

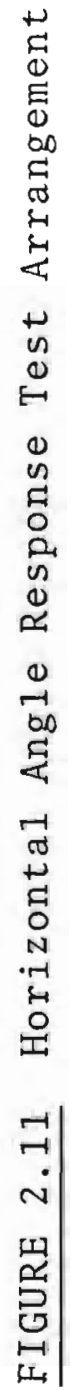




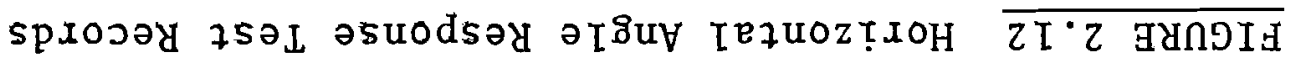

(Spu03әS) วய!!

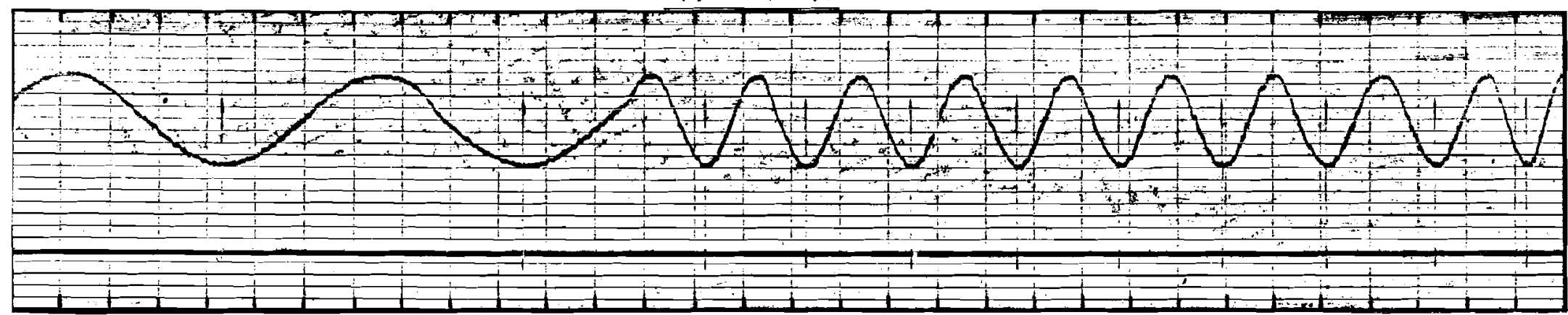

in
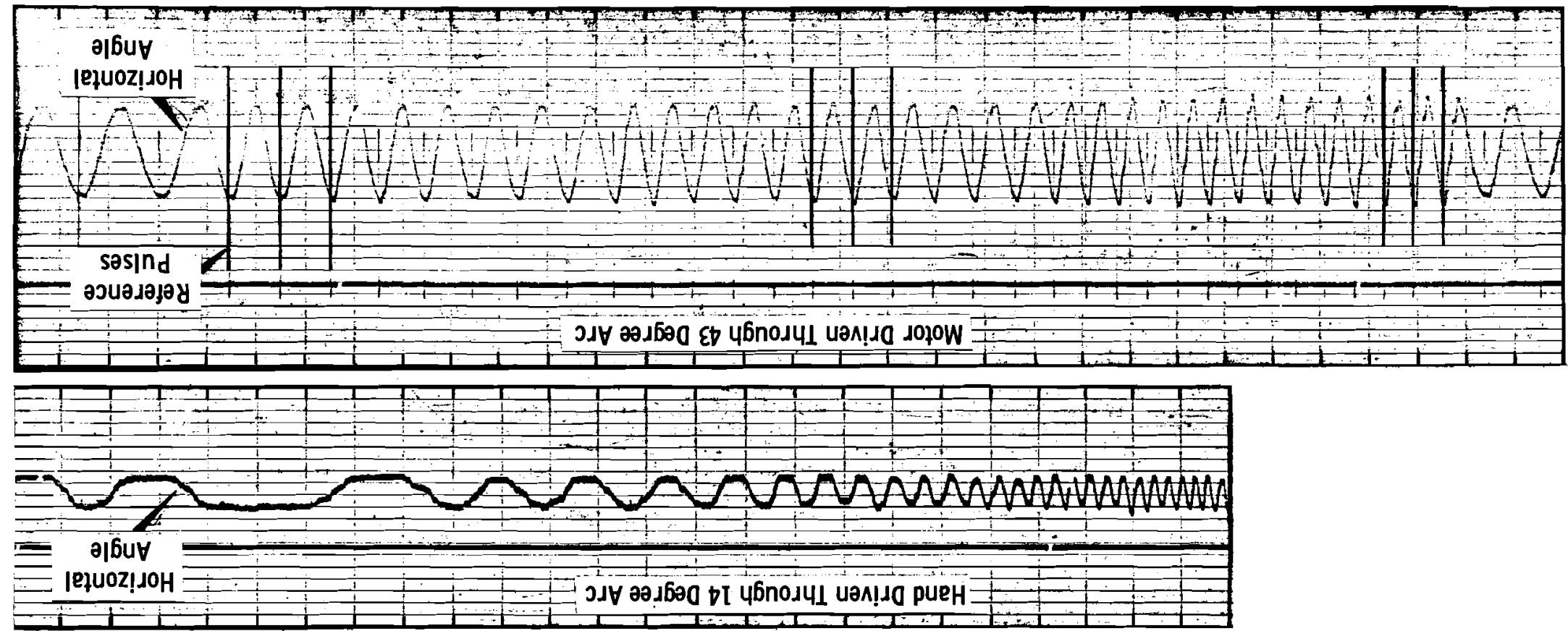
OPERATION

(a) Summer

$\mathrm{R}_{3}$

$R_{3}$

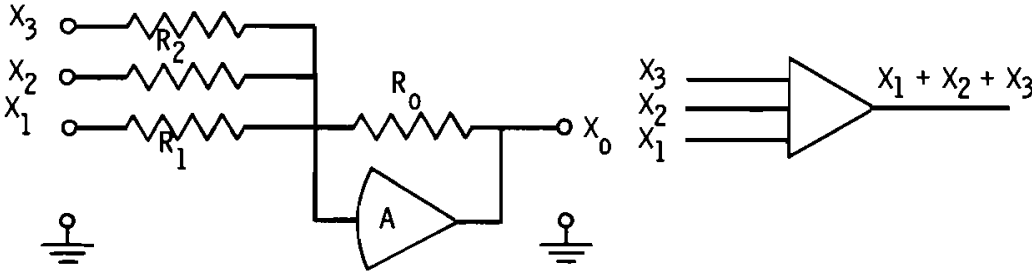

(b)
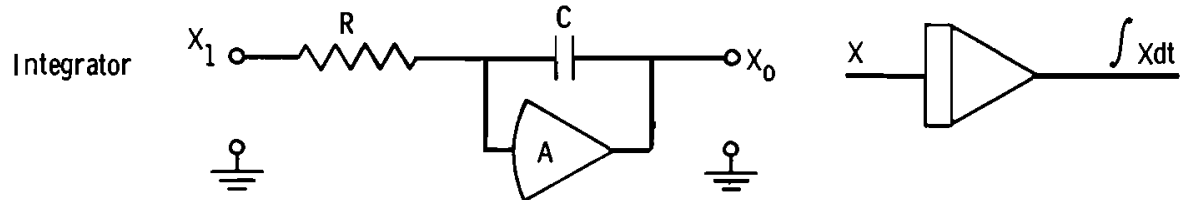

(c) Multiplication by Constant
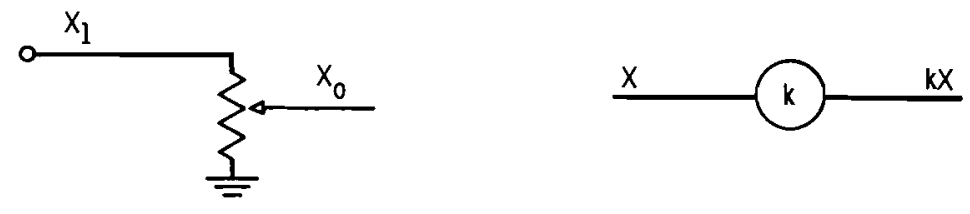

(d) Multiplication of Two Variables

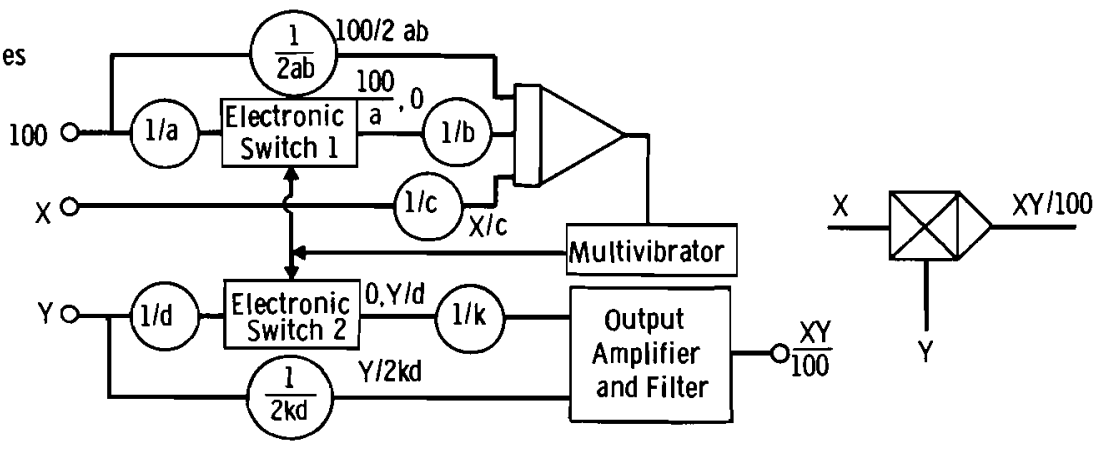

(e) Function Generator
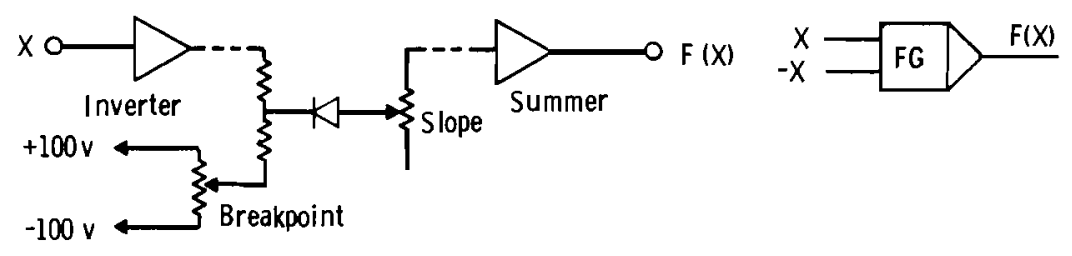

(f) Resolver
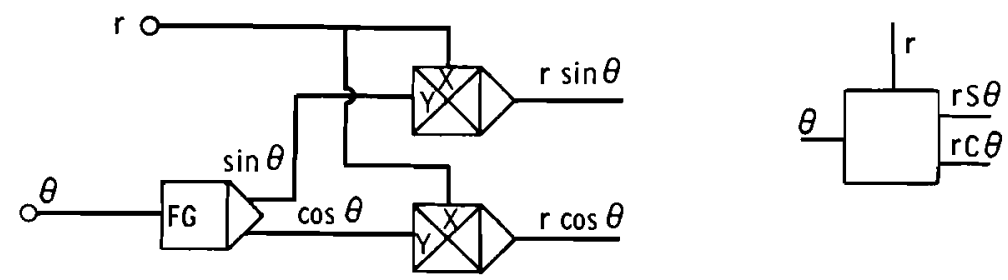

FIGURE 2.13 Basic Analog Computer Components and Symbols 
III. THEORETICAL BACKGROUND FOR ANALYSIS

In $1938, \mathrm{G}$. I. Taylor 43 introduced the concept of analyzing the energy in turbulent wind fluctuations for spectral content through Fourier transform relationships. Since then, this mathematical technique has been used for analyzing random phenomena of widely varying physical origins. A discussion of Wiener's generalized harmonic analysis, upon which this technique is based, and its application to randomly fluctuating electrical siqnals such as those analyzed in the present study is given by Y. K. Lee.19 The limitations and problems introduced by taking finite random time series samples of varying length has been investigated by Blackman and Tukey $^{3}$ leading to more meaningful analysis of experimental data. Mention will be made here briefly of the relationships involved in the analysis of turbulent wind fluctuation signals, and limitations introduced. A more thorough review of the mathematical background presented by Lee and by Blackman and Tukey is covered in Appendix A. When a random time series, $f(t)$, is filterød, it can be shown (Lee ${ }^{19}$ ) that the mean squared filter output is:

$$
\overline{f_{0}^{2}(t)}=\int_{-\infty}^{\infty}|Y(\omega)| 2 \Phi_{11}(\omega) d \omega
$$

where $\Phi_{11}(\omega)$ is the true spectral density distribution for the random time series and $Y(\omega)$ is the transfer or system function for the filter determined from the ratio of the 
complex input and output amplitudes for a steady state sinusoidal signal. Thus the mean square output of the filter is an area under the true spectral density function curve, specified by the shape of the filter. If the filter is constructed to provide low pass or hiqh pass filtering, the mean square output is simply the variance of the original signal with the high end or low end, respectively, of the snectrum eliminated. The sharpness of the frequency cut-off and the range of frequencies eliminated can be selected in the construction of the filter. Similarly, for a band pass filter where the mean square output includes only a portion of the spectral density over a narrow range of frequencies, the center frequency and shape of the filter transfer function can be varied in the construction of the filter.

When two different random functions are filtered separately with matched filters, the mean product of the outputs provides an estimate of the cospectral density, i.e.,

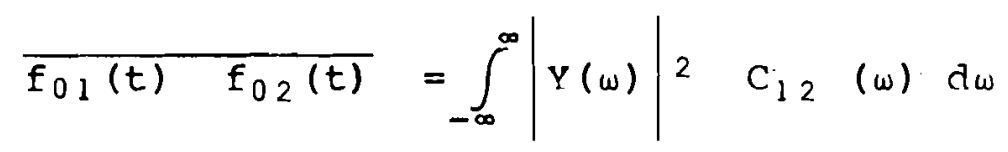

where the transfer function for the filter is again $Y(\omega)$ and the cospectral density, the real part of the cross spectral density function, is qiven by $C_{12}(w)$. Aqain, hiqh or low frequency portions of the area under the cospectral density curve can be eliminated or the contribution from only a narrow band of frequencies can be selected by the proper 
construction of the filter to provide a specified filter transfer function.

For the "direct or heterodyning technique" of spectral analysis, the Fourier transform of a transient function produced by truncating the original time series is taken. Such a truncation can result from simply limiting the length of record as described by Blackman and Tukey ${ }^{3}$. Similarly, the product of the time series and the unit response function of a filter is a transient function and its transform is

$$
\hat{F}_{1}(\omega)=\int_{-\infty}^{\infty} w(s) f_{1}(t-s) e^{-i \omega s} d s
$$

The averaged product of this Fourier transform with the conjugate of the transform of a second truncated random time series gives

$$
\Phi_{12}(\omega)=\int_{-\infty}^{\infty} \mathrm{H}(\omega-\xi) \Phi_{12}(\xi) \mathrm{d} \xi
$$

providing estimates of the power spectral density when the two random functions are the same, and cospectral and quadrature spectral density when they are different. Here, $H(\omega)$ is the power transfer function for the low pass filters and $\Phi_{12}(\omega)$ is the true spectral density function. The convolution integral of Equation (3.4) is of the same form as the spectral estimates obtained through the Fourier transform of the autocovariance function for a random time series with a 
finite length of record, described by Blackman and Tukey ${ }^{3}$. The relationships described in the foreqoing discussion can provide the basis for a number of spectral analysis techniques, some of which will be included in the analog computer program discussion to follow. 
IV. DATA ANALYSIS PROGRAMS

In proceeding from the raw data to the analyzed results the sequence is as follows:

(a) Coordinate transformation changes the data from a polar frame to a cartesian frame in which the mean wind is aligned with the $x$ axis.

(b) Reynolds' stresses are computed in the new frame of reference.

(c) Power spectra for the fluctuations of all three wind components are computed.

(d) Cospectra for pairs of the three wind components are computed.

\section{Coordinate Transformation Program}

The analog computer has been proqrammed, first of all, to take the tape recorded inputs (see section $V$ ) and to provide the wind fluctuation components $u^{\prime}, v^{\prime}, w^{\prime}$ for recording on magnetic tape. Figure 4.1 shows the diagram for this program into which the wind component meter signals, originally tape recorded at $3-3 / 4$ inches per second (ips), are played back at 60 ips. The program provides for the calibrations of the turbulence instrument and transforms coordinates from spherical to cartesian. A mathematical rotation of coordinates is made so that the $\mathrm{x}$ coordinate is directed along the direction of the mean wind. The mean wind is removed and the three wind fluctuation components are 
re-recorded at $3-3 / 4$ ips, thus contracting the record to 1/16th of the oriqinal length.

The first part of the coordinate transformation pro-gram, shown in Fiqure $4.1 \mathrm{~d}$, is a preconditioning circuit, to compensate for differences in the calibration of the two tape recorders involved in handling the data. Such differences can easily occur, particularly since different tape speeds as well as different tape recorders are used in recording and playback. Calibration signals of zero and one volt, recorded on each channel of the original tape before or after the data are played back into the preconditioning circuit. Bias signals are added to the incoming zero calibration signals by adjusting potentiometers $h 0$, $\mathrm{h} 1$, and $\mathrm{h} 2$ until the outputs of amplifiers $\mathrm{A} 0, \mathrm{~A} 4$, and A 8 are zero. Then, with the one-volt calibration signals as inputs a gain adjustment is made for each channel with potentiometers $h 3, h 4$, and $h 5$ until the outputs of $A 1$, $A 5$, and $A 9$ reach one volt. In the speed signal channel a bias voltage is added at $A$. This replaces a dc level which had been removed from the instrument speed siqnal during recording so that the recorded signal would be centered in the input range of the tape recorder. Now when the data are played back they are presented to the computer beyond the preconditioning circuit, just as they were generated by the wind component meter. Also included in the preconditioning circuit are diode limiters at $A$, A 4, and A 8. These 
prevent the magnitude of the incoming signals from reaching a level that would overload the computer amplifiers. By proper adjustment extraneous noise pulses are kept at a harmless level while legitimate signals are passed unaltered.

The next stage in the program provides for the calibration of the wind component meter. The instrument speed signal is the input to function generator 12 which is adjusted to generate the speed calibration curve as shown in Figure 4.2 so that its output voltage is directly proportioned to the instantaneous speed. The initial breakpoint and slope are determined from the -100 volt input to $A 52$ and the setting of potentiometer $h 6$, respectively.

The calibration of the vertical angle at a wind speed of 5 meters per second is set in function generator 10. The initial breakpoint and slope are set with potentiometers h 9 and h 10, respectively, and the other points, set in the function generator, are shown in Figure 4.3 . Then the output in volts from amplifier 50 is equal to the vertical angle in degrees for any instrument signal input when the wind speed is $5 \mathrm{mps}$. As the wind speed varies from $5 \mathrm{mps}$ a correction is made to the vertical angle resulting from the change in the calibration of the vertical angle with change in wind speed. Function generator 11 , set up as shown in Figure 4.4 and with initial breakpoint and slope given by $h 7$ and $h 8$, produces a signal proportional to $\frac{\Delta \phi}{\Delta V}$ for any

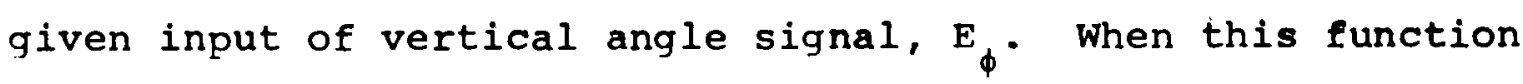


generator output is multiplied in $M 30$ by the deviation in wind speed from 5 mps supplied from $A 32$, the vertical angle correction, $\Delta \phi$, is available to be added in amplifier 18 to $\phi_{0}$, producing a signal proportional to the true vertical angle regardless of wind speed.

The vertical angle is supplied through a trunk line to the sine and cosine function generator in the resolver of a second analog computer, the Fase 2133, since only one resolver is provided in the Ease 1132 and it is used in another part of the program. The sine and cosine of the vertical angle is returned through trunk lines to the Fase 1132 where it is multiplied by the wind speed in $M 28$ and $M 29$ to give the vertical wind component, $w$, and the wind speed, in the horizontal plane, $\mathrm{V}_{\mathrm{H}}$. The horizontal angle (directly proportional to the instrument signal so that no function qenerator is reguired) and the horizontal wind speed are supplied to the resolver in the Ease 1132 which provides the horizontal components, $\mathrm{u}$ and $\mathrm{v}$, as outputs. It is necessarv to scale the inputs to both resolvers in order that the outputs $u, v$, and $w$ give one volt for each meter per second.

The average of each of the components is obtained from the outputs of integrators A 35, A 38, A 20, and A 21 . The magnitude and direction of the mean wind is determined from these and the coordinate system is reoriented with the $x$ axis directed along the mean wind. To do this, the test is played back through the computer again and the mean horizontal 
and vertical angles are subtracted in amplifiers $A$ and A 18, respectively, by adjusting potentiometers $P$ and $\mathrm{P}$ 18. The mean wind speed is also subtracted from the longitudinal component at A 30 by setting potentiometer $P 94$. Then the fluctuation components, $u^{\prime}, v^{\prime}, w^{\prime}$ with all mean values removed, are recorded on magnetic tape at 3-3/4 ips after attenuation with potentiometers $P 41, P 42$, and $P 43$ to maintain the inputs to the tape recorder within an acceptable range. The coordinate transformation is monitored on a Brush Chart Recorder for each test. Typical results are shown in Figure 4.5 .

\section{Reynolds Stress Program}

The turbulence component signals, recorded after coordinate transformation, are played back later into a number of analog computational programs to investigate the properties of the turbulence. The first program determines the variances of the turbulence components and the covariances between them, i.e., the Reynolds stresses. Figure 4.1 shows the diagram for the program. After calibrating the program for any two of the signals through the preconditioning circuit with the zero and one volt calibration signals recorded on each channel of the tape with the data, each channel of data is passed through an active high pass filter to remove very long period fluctuations. Each filter is constructed from a high gain dc amplifier with an input 
resistance and capacitance in series and with resistive feedback. The operation of this arrangement is described by a first order, linear, ordinary differential equation. The transfer function for such a linear system at any given frequency is defined by the ratio of the complex input and output amplitudes for a steady state sinusoidal signal and is the Fourier transform of its unit impulse response. (Lee ${ }^{19}$ ) For this arrangement the transfer function is

$$
Y(\omega)=\frac{i \omega R_{2} c_{1}}{i \omega R_{1} c_{1}+1}
$$

For the values of the components used in this filter,

$$
Y(\omega)=\frac{i \omega}{i \omega+0.2}
$$

The power transfer function is

$$
|Y(\omega)|^{2}=\frac{\omega^{2}}{\omega^{2}+.04}
$$

so that the half-power point is at $0.032 \mathrm{cps}$. Most of the data have been analyzed with a playback tape speed of $3-3 / 4$ ips so that a factor of 16 remains between the analysis and original fluctuation frequencies. Thus in terms of the true frequency of the atmospheric fluctuations, the half-power point occurs at $0.002 \mathrm{cps}$, so that the filter nominally passes fluctuations with periods smaller than $8-1 / 3$ minutes. The $80 \%$ and $20 \%$ power transmission points are at frequencies a factor of two on either side of the half-power point. 
After filtering, the square of each of the signals is taken and the cross product is calculated in multipliers M 9, M 11, and M 10, respectively. The integrals of the filtered signals, their squares and their cross product are taken over the duration of each test. Dividing the final outputs from A 20, A 28 and A 24 by the time, the Reynolds' stresses are given. The integration begins when the computer is put in the "compute" mode. This occurs simultaneously, through a switching arrangement in the preconditioning circuit, with the introduction of the data into the program. Similarly, stopping the integration with the "hold" mode occurs simultaneously with removing the data. Figure 4.7 shows an example of a monitored calculation.

Two modifications of this procedure should be mentioned. The first technique attempted did not use the filter discussed above, but extraneous dc levels in the signals were removed by supplying a dc bias after the average signal levels were determined at $A 21$, and $A 25$. This involved considerable recalculation to remove exactly the contribution of dc levels in the Reynolds' stress calculations and was found to be impractical whereas the high pass filter allowed the calculations to be made quite rapidly. The other variation was in the filtering used for cases above 12 meters. The playback speed used for the analysis was $7-1 / 2$ ips rather than $3-3 / 4$ ips so that the half-power point of the filter occurred at a real time 
frequency of 0.001 cps. This filtering essentially passed fluctuations with periods less than $16-2 / 3$ minutes so that larger eddies, more important at greater heights, were included in the stress calculations. This proved to be important for data gathered at 12.2 meters. See section VII.

\section{Power Spectral Program}

The power spectral estimates are obtained with a program, shown in Figure 4.8, based on Equation (3.1) utilizing eighteen active band pass filters. The turbulence signals are played back one at a time into the program, which includes, first of all, a preconditioning circuit. As before, the program is calibrated with the zero and one-volt signals accompanying the data. In addition, a switch and another gain calibration potentiometer are provided to allow a gain selection during the analysis. Since both the length of the tests and the average level of the signals varies among tests, the gain selection optimized the program operation without the necessity of re-scaling the entire program for each test. Another switching arrangement in the preconditioning circuit introduces the data into the program and simultaneously starts the integration of the results. Another position of the switch simultaneously removes the data and puts the computer in "hold," stopping the integration and retaining the final values at the integrator outputs. Beyond the preconditiong circuit, the program consists 
of eighteen filter channels, each channel including a buffer amplifier, a prefilter gain adjustment potentiometer and amplifier, the filter with a compensation potentiometer inserted, a multiplier, a scaling potentiometer and an integrator. The channels differ only in the values of the filter components which determine different center frequencies and filter widths, in the values of the filter compensation potentiometer settings, and in the other potentiometer settings for a few cases when it is necessary to rescale only a portion of the channels. The construction of the filter (Cummins ${ }^{8}$ ) is shown only for the first channel but in every case it is a second order filter, the operation of which is described by a second order linear ordinary differential equation which yields a transfer function of the form

$$
Y(\omega)=\frac{K}{1+1 Q\left(\frac{\omega}{\omega_{0}}-\frac{\omega_{0}}{\omega}\right)}
$$

where the gain of the filter is $K=\frac{R_{2} C_{1}}{R_{1}\left(C_{1}+C_{2}\right)}$

the center frequency is $\omega_{0}=\frac{1}{\sqrt{R_{1} R_{2} C_{1} C_{2}}}$

and a measure of the width is $Q=\frac{1}{\omega_{0} R_{1}\left(C_{1}+C_{2}\right)}$ 
The power transfer function is

$$
|Y(\omega)|^{2}=\frac{K^{2}}{1+Q^{2}\left(\frac{\omega}{\omega_{0}}-\frac{\omega_{0}}{\omega}\right)^{2}}
$$

With the approximation that

$$
\frac{\left(\omega+\omega_{0}\right)\left(\omega-\omega_{0}\right)}{\omega \omega_{0}}=\frac{\Delta_{\omega}}{\omega_{0}}
$$

Where

$$
\Delta^{\prime} \dot{w}=2\left(w-\omega_{0}\right) \text {. }
$$

we can closely approximate this relationship with

$$
|Y(\omega)|^{2}=\frac{K^{2}}{1+Q^{2}\left(\frac{\Delta \omega}{\omega_{0}}\right)^{2}}
$$

from which it is apparent that the half-power point occurs when

$$
\Delta \omega_{\mathrm{HP}}=\frac{w_{0}}{Q}
$$

demonstrating that $Q$ is a measure of the filter width as well as showing that the width is proportional to the center frequency. In this case the filter was designed for $Q=10$ so that the width would always be $1 / 10$ of the center frequency. Since the filters are constructed with non-precision components the actual widths and gains deviate somewhat from the design values and were determined experimentally by passing sinusoidal signals at a number of frequencies through each filter and then comparing the input with the output 
amplitudes. The center frequency, $\mathrm{n}_{\mathrm{O}}$, the gain, $\mathrm{K}$, the width as indicated by the value of $Q$, and the area, $A=\frac{1}{K^{2} \omega_{0}} \int_{-\infty}^{\infty}|Y(\omega)|^{2} d \omega$ under the power transfer function curve normalized to the gain and center frequency, are given in Table 4.1 for each filter in the program as determined from testing the filters. The filters overlap each other close to the one-quarter power point rather than the more ideal half-power point. Also, the total range of frequencies covered is somewhat limited for investigating atmospheric turbulence spectra. However, playing back the data at more than one tape speed allows the program to sample the spectrum for a number of over-lapping intervals. The output of each filter, squared in the multiplier, multiplied by the filter compensation potentiometer value, $R$, as well as a scaling factor, P, determined from the other potentiometer settings and amplifier gains, and integrated over the length of the test is available at the output of the integrator for each channel. The final integral, I, divided by the integration time give, according to Equation (3.1)

$$
\frac{I}{T}=\overline{f_{0}^{2}(t)}=P R \int_{-\infty}^{\infty}|Y(w)|^{2} \phi_{11}(w) d w
$$

Assuming that the spectral density is essentially constant over the narrow range of frequencies passed by the filter, we can write 
$\frac{I}{T}=P R \Phi_{11}(\omega) \int_{-\infty}^{\infty}|Y(w)|^{2} d \omega=P R A K^{2} \omega_{0} \Phi_{11}(\omega)$

Then, considering the gain, G, introduced in the preconditioning circuit and the attenuation, $B$, of the turbulence signals, introduced during recording and determining the number of volts for each meter per sec of wind speed, the product of the true wind fluctuation frequency, $n$, and the spectral density of the wind fluctuations, $S(n)$, is, in units of (mps) ${ }^{2}$

$$
\mathrm{nS}(\mathrm{n})=I / \mathrm{T} P \mathrm{R} A \mathrm{~K}^{2} \mathrm{G}^{2} \mathrm{~B}^{2}
$$

The filter compensation potentiometer setting, $R$, is adjusted to account for the variations in the filter area normalized to frequency, $\mathrm{AK}^{2}$. Then the spectral estimates are found by simply multiplying all the integrals for any given test by a single factor. Figure 4.9 shows a monitored power spectral calculation.

The calculations for some of the first tests analyzed obtained integrals proportional to $S(n)$ rather than $n s(n)$ by including in the filter compensation potentiometer setting an additional factor inversely proportional to frequency. This calculation proved less effective than the final technique since the integrals covered a wide range of magnitudes, large enough to approach the overload limit of the computer for the low frequency estimates and small enough at high 
frequencies to approach the noise level of the equipment. To obtain the true spectral density, it was necessary to multiply the analysis spectral density by the data time base change factor: 256 for the playback speed of 60 ips into the filter program and 32 for the $7-1 / 2$ ips playback speed. For the later calculations of $\mathrm{nS}(\mathrm{n})$ no such factor was required since the product of analysis frequency and analysis spectral density equals the product of the frequency and true spectral density regardless of the tape speed.

The integrals for the highest frequencies in the analysis are sometimes small enough to contain a considerable error resulting principally from the multiplier drift. Numerous checks are made during the analysis period, obtaining integrals with no input into the program. These "noise integral" checks are compared with the test results and where the noise integral introduces an error exceeding 108 of the test integral the spectral estimate is eliminated. other errors introduced in this analysis stem first from only being able to calibrate the program to within plus or minus one or two per cent of the one-volt calibration. Also, small differences in the length of record reproduced for each part of the analysis of a given test contributes to small differences in the spectral estimates for the same frequencies in the range where the analysis results overlap. Finally, the determination of the true filter areas were made to within about plus or minus five per cent, contributing 
errors of the same magnitude in the spectral estimates.

Cospectral Program

The cospectral filter program, based on Equation (3.2), is constructed similarly to the power spectral program and is shown in Figure 4.10. A preconditioning circuit calibrates the program, provides a gain selection and switches the two channels of data and the integration on and off simultaneously. Eleven dual filter channels follow, each with a buffer amplifier and scaling potentiometer for each data leg of the channel, a pair of matched filters--one for each of the two signals--a multiplier, a filter compensation potentiometer and an integrator. The filters are again second order with the same design as the filters for the power spectral program. All filters were again tested with sinusoidal signals of various frequency. The filters are well matched so that the shape of the power transfer-function, determined from the output of the multiplier for the various sinusoidal inputs, is quite similar to the design function shape. However, the gain and the width of each filter again differ somewhat from the design values. The filter test results were again used to determine the true values of $k^{2}$ and $Q$. These, the center frequencies and the power transfer function area, normalized to gain and center frequency are tabulated in Table 4.2 for each of the eleven filters. The final integral for any channel divided by the time 
of integration is, from Equation (3.2)

$$
\frac{I}{T}=\overline{f_{10}(t) f_{20}(t)}=p R \int_{-\infty}^{\infty}|Y(\omega)|^{2} C_{12}(\omega) d_{\omega}
$$

Assuming the cospectral density to be relatively constant over the narrow frequency range of filter, we have

$$
\frac{I}{\mathrm{~T}}=\mathrm{P} \quad \mathrm{R} \quad \mathrm{A} \quad \mathrm{K}^{2} \quad \omega_{0} \mathrm{C}_{12}(\omega)
$$

Accounting for the selectable qain and the attenuation giving the number of volts per mps introduced in recording the analyzed wind component fluctuations, the true cospectral estimates are given in $(\operatorname{mps})^{2}$ by

$$
\mathrm{nC}(\mathrm{n})=\mathrm{I} / \mathrm{T} P \mathrm{P} A \mathrm{~K}^{2} \mathrm{G}^{2} \mathrm{~B}^{2}
$$

Again, the filter compensation potentiometer, R, is adjusted in each channel inversely proportional to $A K^{2}$, the filter area, normalized to center frequency so the final integrals for a given test are all multiplied by a single constant. For the first tests, analyzed for cospectral density, $c(n)$, rather than $\mathrm{nC}(\mathrm{n})$, the filter compensation potentiometer setting included a factor to remove the dependence of the filter width on center frequency. Later, this dependence was retained, yielding estimates of $\mathrm{nC}(\mathrm{n})$ with a narrower range of output levels. The errors introduced in this program are of the same type as those found in the power spectral analysis program and of the same magnitude, generally on the order of five to ten per cent. The example of the cospectral filter 
outputs, Figure 4.11, resulted from the first arranqement calculating $C(n)$.

\section{Alternate Spectral Density Program}

Although the following program has not been used in computing the results to be presented in this paper, it has been tested and shown capable of determining power spectral and cross spectral estimates. It uses heterodyning and filtering, followed by multiplication and averagina. It is an electrical analog of the procedure described in Equations (3.3) and (3.4) and in the associated discussion.

Figure 4.12 shows two wind component signals, after preconditioning, each being multiplied by the sine and cosine of a given frequency, $w$. The products are each subjected to low pass filtering giving continuously the real and imaginary parts of the Fourier transforms of the oriqinal signals. The outputs from A 51 and A 12 are the imaginary parts of each Fourier transform. The sum of the squares of the real and imaginary parts for each component form the power spectral estimates at the output of $A 18$ and $A 26$. The cross product of the real parts summed with that of the imaginary parts provides the cospectral estimates at $A$ 19. The cross product of the real part for one component with the imaginary part for the other component, summed with the other like product provides the quadrature spectral density at the output of amplifier A 22. Statistically stable estimates are 
obtained by smoothing the spectral densities and recording the continuous results at the outputs of $A 24, A 29, A 25$, and $A 28$, as well as integrating over the length of record at $A 30, A 35, A 31$, and $A 34$, to obtain single average values for the entire test. An example of the recorded continuous calculation is given in Figure 4.13. With additional equipment including a tape loop mechanism and an oscillator that can be continuously swept slowly through a range of frequencies, it will be practical to use this program in place of the multiple filter programs to provide the power spectral and cospectral distributions as well as quadrature spectral distributions not presently obtained.

\section{Statistical Reliability of Spectral Estimates}

Blackman and Tukey have demonstrated that, assuming a Gaussian process, the spectral estimates follow a chisquare distribution with the number of degrees of freedom, $k$, equal to the number of elementary frequency bands in the equivalent width of the spectral window, for a relatively smooth spectral distribution. Taking the width of the filter at the half-power point as the equivalent width, $w_{e}$ and $1 / 2 \mathrm{~T}$ as the width of the elementary frequency bands, the number of degrees of freedom is

$$
k=\frac{W_{e}}{1 / 2 T}=\frac{\Omega_{0} / Q}{1 / 2 T}=\frac{2 \mathrm{Tn}_{0}}{Q}
$$


T'able 4.3 gives the number of degrees of freeciom for each test at the lowest frequency of the analysis where the filter is narrowest and consequently the spectral estimate is least stable, at the high frequency end of the analysis where the estimates are most stable, and at a frequency in the middle of the range of analysis. Also given in the table are the ratios of possible spectral estimates to true spectral density between which the estimates will fall with 808 confidence. Table 4.3 also applies to the cospectral estimates since the same general range of frequencies was covered and the filter design was the same as for the power spectral estimates. The variability for both spectral and cospectral estimates was found using the design width parameter, $Q=10$. This resulted in slightly smaller values for the number of degrees of freedom than the somewhat smaller width parameters, found in the filter tests, would have given. Consequently, the estimates are slightly more stable than indicated by the calculation. 
TABLE 4.1

\section{POWER SPECTRAL PROGRAM PARAMETERS}

\begin{tabular}{|c|c|c|c|c|}
\hline Filter & $\begin{array}{c}\mathrm{n}_{0} \\
\text { (cps) }\end{array}$ & $\mathrm{K}^{2} \times 10^{-4}$ & $Q$ & $\mathrm{~A}$ \\
\hline 1 & 3.10 & 3.67 & 9.07 & .173 \\
\hline 2 & 3.90 & 3.93 & 9.56 & .164 \\
\hline 3 & 4.70 & 3.24 & 8.10 & .194 \\
\hline 4 & 6.15 & 3.80 & 9.59 & .164 \\
\hline 5 & 8.00 & 4.08 & 9.39 & .167 \\
\hline 6 & 9.62 & 3.06 & 8.79 & .179 \\
\hline 7 & 12.4 & 2.43 & 7.59 & .207 \\
\hline 8 & 15.9 & 2.99 & 9.54 & .165 \\
\hline 9 & 18.0 & 3.24 & 9.35 & .168 \\
\hline 10 & 24.5 & 3.06 & 9.04 & .174 \\
\hline 11 & 30.9 & 2.99 & 7.81 & .201 \\
\hline 12 & 38.8 & 3.53 & 9.29 & .169 \\
\hline 13 & 50.3 & 2.99 & 8.87 & .177 \\
\hline 14 & 66.7 & 1.44 & 5.89 & .267 \\
\hline 15 & 77.2 & 3.61 & 8.79 & .179 \\
\hline 16 & 99.2 & 2.40 & 6.77 & .232 \\
\hline 17 & 124. & 2.56 & 7.00 & .224 \\
\hline 1.8 & 158. & 4.00 & 8.47 & .185 \\
\hline
\end{tabular}


TABLE $\quad 4.2$

COSPECTRAL PROGRAM PARAMETERS

\begin{tabular}{|c|c|c|c|c|}
\hline Filter & $\begin{array}{l}n_{0} \\
\text { (cps) }\end{array}$ & $\underline{\mathrm{K}^{2} \times 10^{-4}}$ & $Q$ & A \\
\hline 1 & 3.70 & 3.97 & 8.69 & .181 \\
\hline 2 & 4.80 & 2.08 & 7.46 & .211 \\
\hline 3 & 6.15 & 2.65 & 8.53 & .184 \\
\hline 4 & 8.85 & 3.53 & 8.89 & .177 \\
\hline 5 & 12.8 & 3.40 & 10.2 & .154 \\
\hline 6 & 20.8 & 3.84 & 8.41 & .187 \\
\hline 7 & 27.5 & 2.32 & 7.86 & .200 \\
\hline 8 & 39.5 & 2.72 & 8.57 & .183 \\
\hline 9 & 54.0 & 1.92 & 7.69 & .204 \\
\hline 10 & 70.0 & 2.56 & 7.83 & .201 \\
\hline 11 & 100 & 3.36 & 5.89 & .267 \\
\hline
\end{tabular}


TABLE 4.3

VARIABILITY OF SPECTRAL ESTIMATES

Length

Test (sec.)

$\begin{array}{rr}2-1 & 1740 \\ 2-2 & 2220 \\ 2-3 & 960 \\ 4-1 & 960 \\ 5-1 & 3840 \\ 5-2 & 1200 \\ 5-3 & 2400 \\ 6-1 & 720 \\ 6-2 & 960 \\ 6-3 & 3540 \\ 7-1 & 3840 \\ 7-2 & 3540 \\ 9-1 & 8040 \\ 11-1 & 3180 \\ 11-2 & 1980 \\ 12-1 & 2700 \\ 12-2 & 2700 \\ 13-1 & 3420\end{array}$

Degree of Freedom

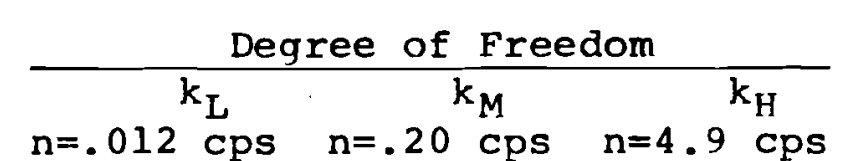

4

5
2

2

9

3

6

2

2

9

9
9

19

8

5

7

7

8

940

940

7870

3120

2640

3350
Limiting Ratios of Estimates to True Spectral Density for $80 \%$ Confidence Interval

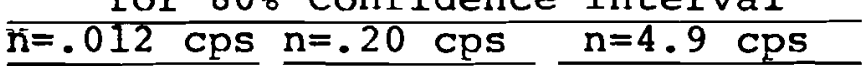

$.26-1.94 .78-1.22>.93-<1.07$

$.32-1.85 .81-1.19>.93-<1.07$

$.10-2.3 \quad .72-1.30>.93-<1.07$

$.10-2.3 .72-1.30>.93-<1.07$

$.46-1.63 .86-1.15>.93-<1.07$

$.19-2.08 .75-1.26>.93-<1.07$

$.36-1.78 .82-1.18>.93-<1.07$

$.10-2.3 \quad .69-1.34>.93-<1.07$

$.10-2.3 .72-1.30>.93-<1.07$

$.46-1.63 .85-1.15>.93-<1.07$

$3760 \quad .46-1.63 .86-1.14>.93-<1.07$

$3460 \quad .46-1.63 .85-1.15>.93-<1.07$

$.61-1.43 .90-1.10>.93-<1.07$

$.44-1.67 .84-1.16>.93-<1.07$

$.32-1.85 .80-1.20>.93-<1.07$

$2640 \quad .40-1.72 .82-1.18>.93 \sim<1.07$

$.40-1.72 .82-1.18>.93-<1.07$

$.44-1.67 .84-1.16>.93-<1.07$ 


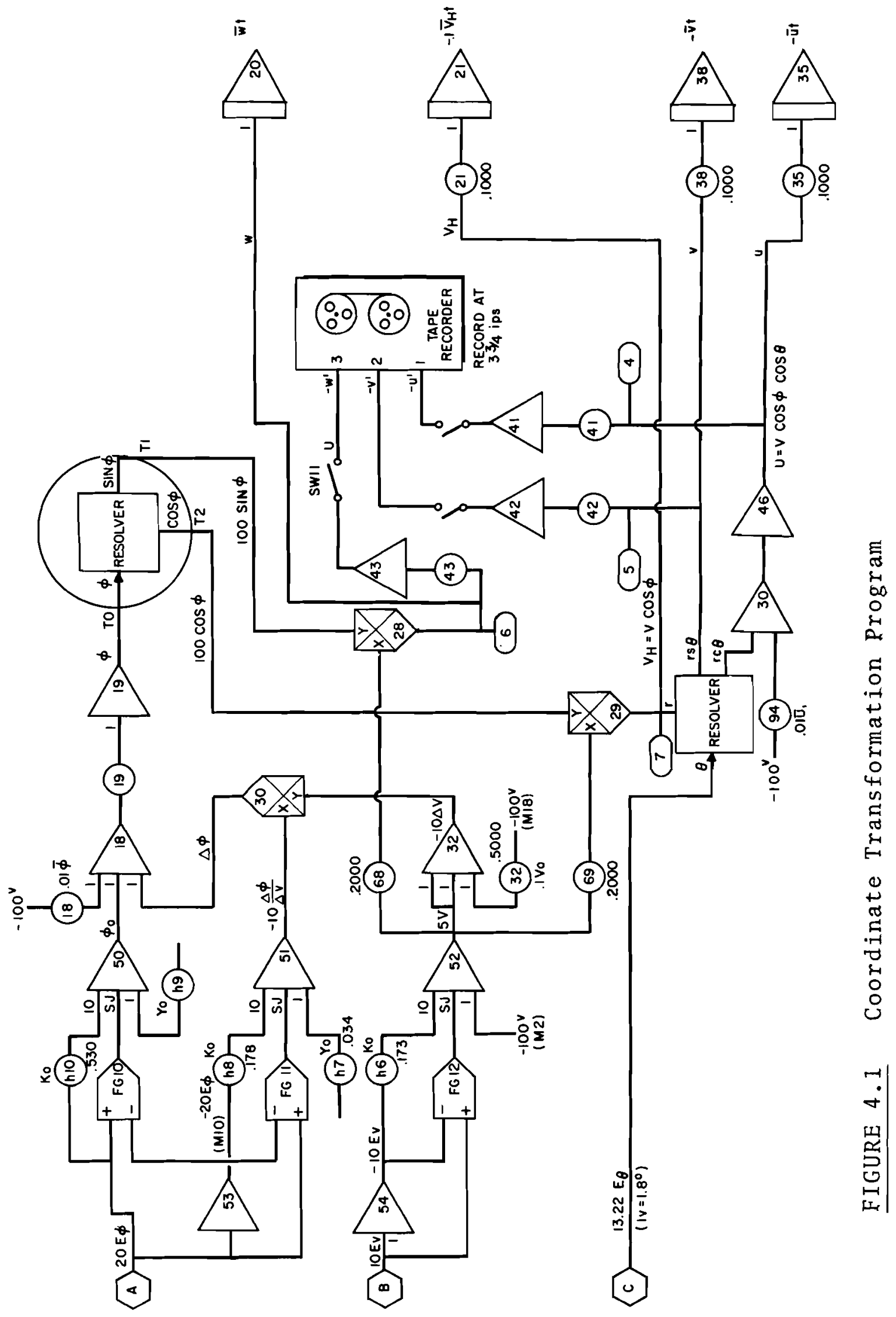




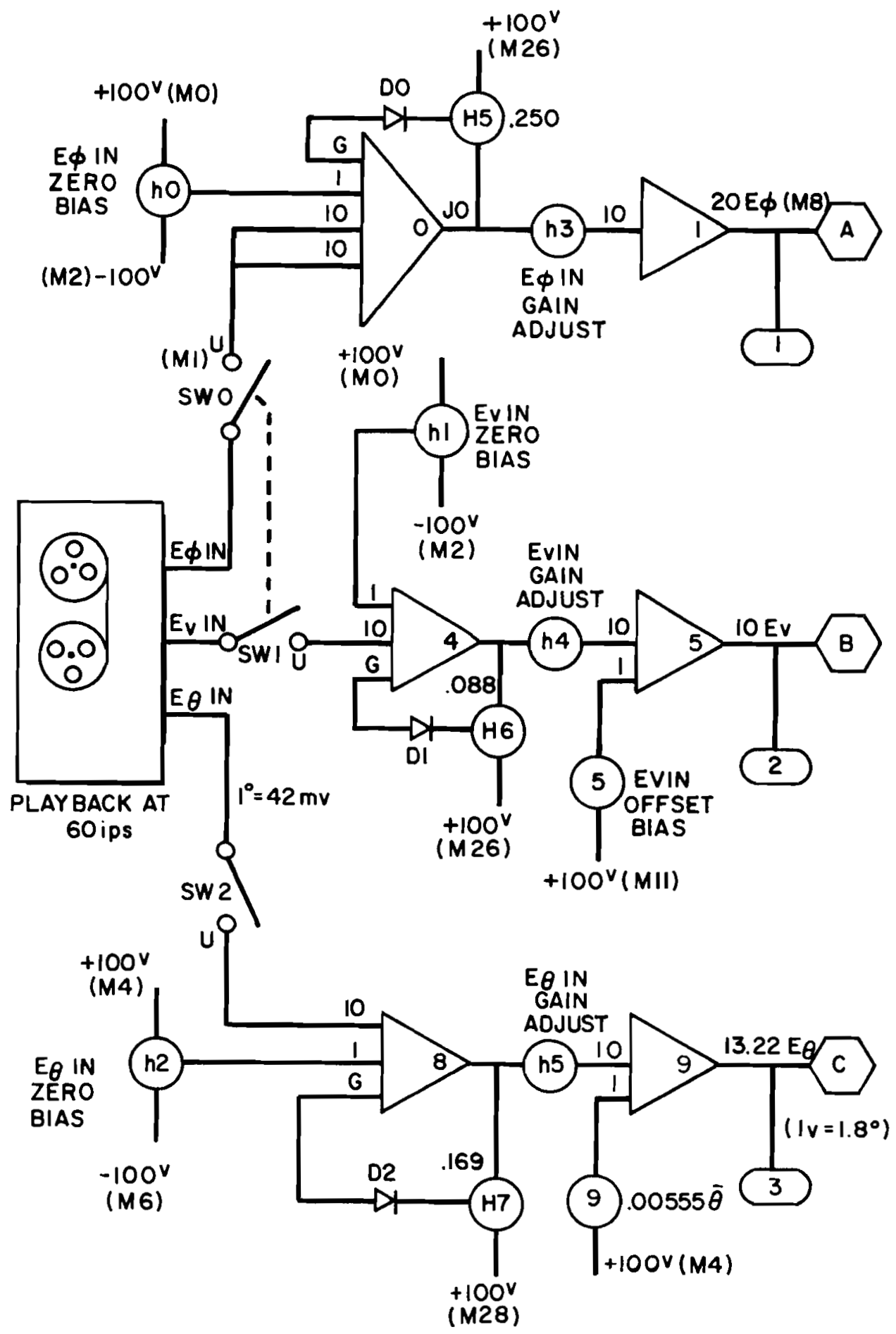

FIGURE $4.1 \mathrm{a}$ Coordinate Transformation Program 


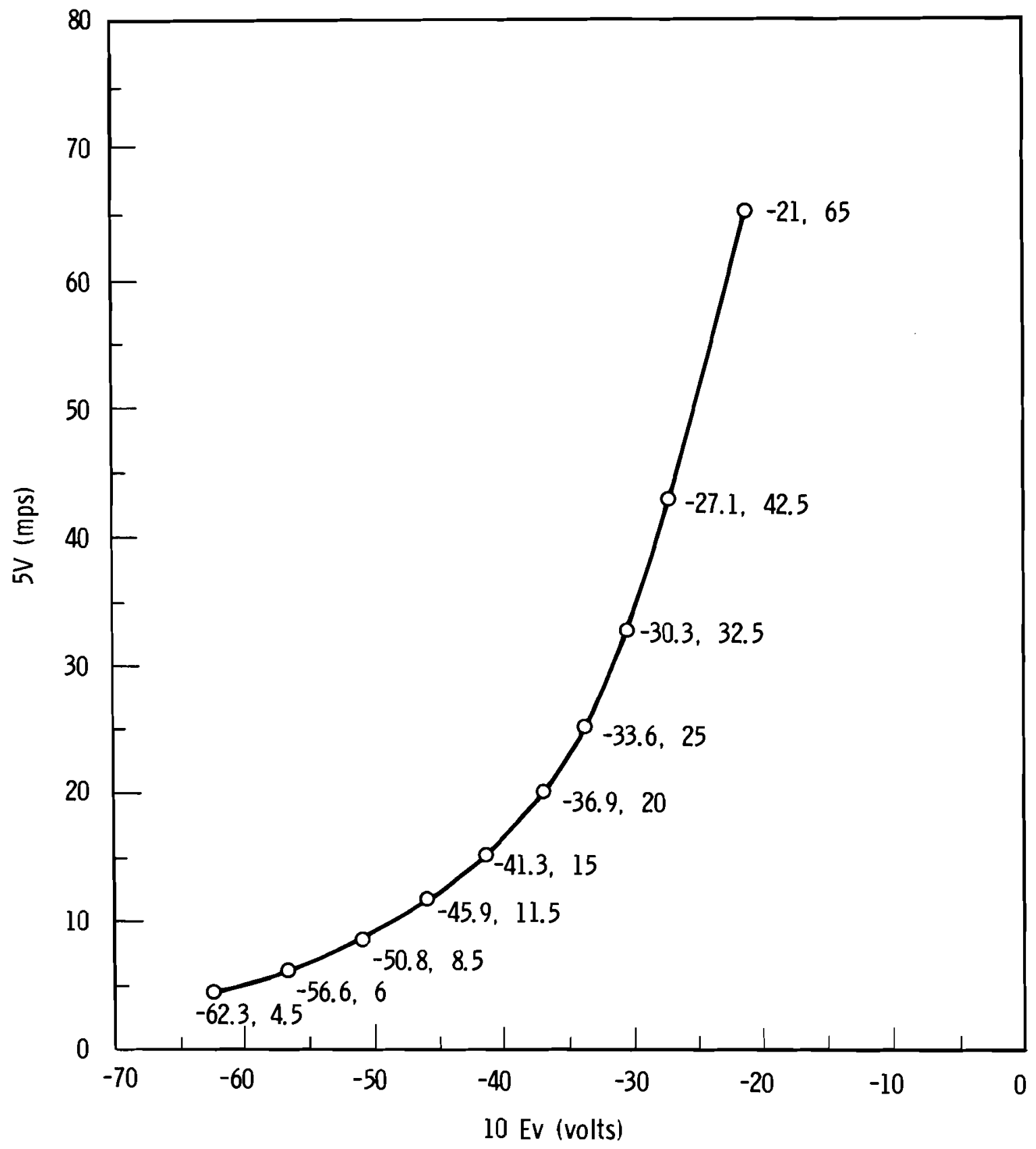

FIGURE 4.2 Function Generator Settings for Speed Calibration 


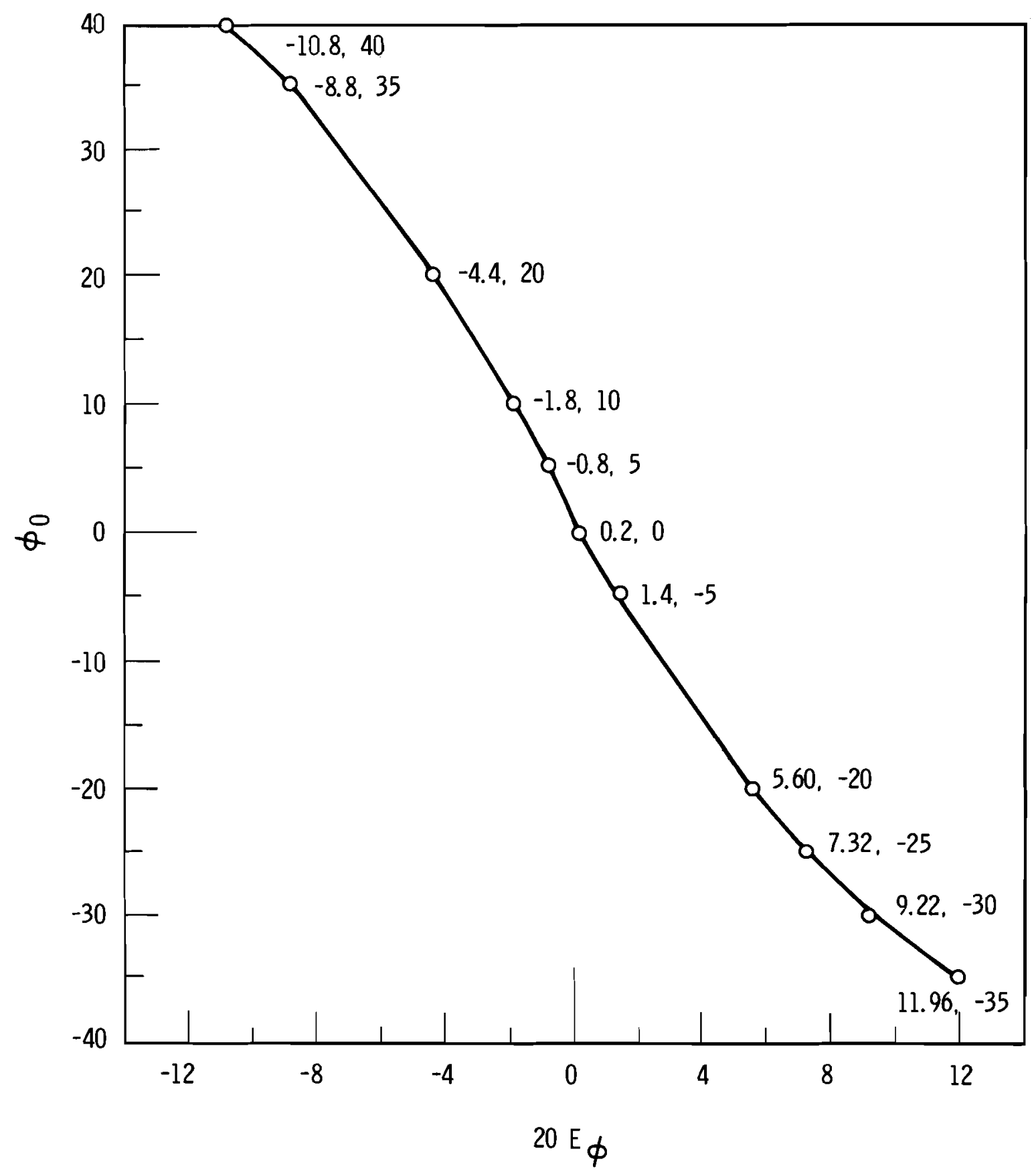

FIGURE 4.3 Function Generator Settings for Vertical Angle Calibration at $5.0 \mathrm{mps}$ 
62

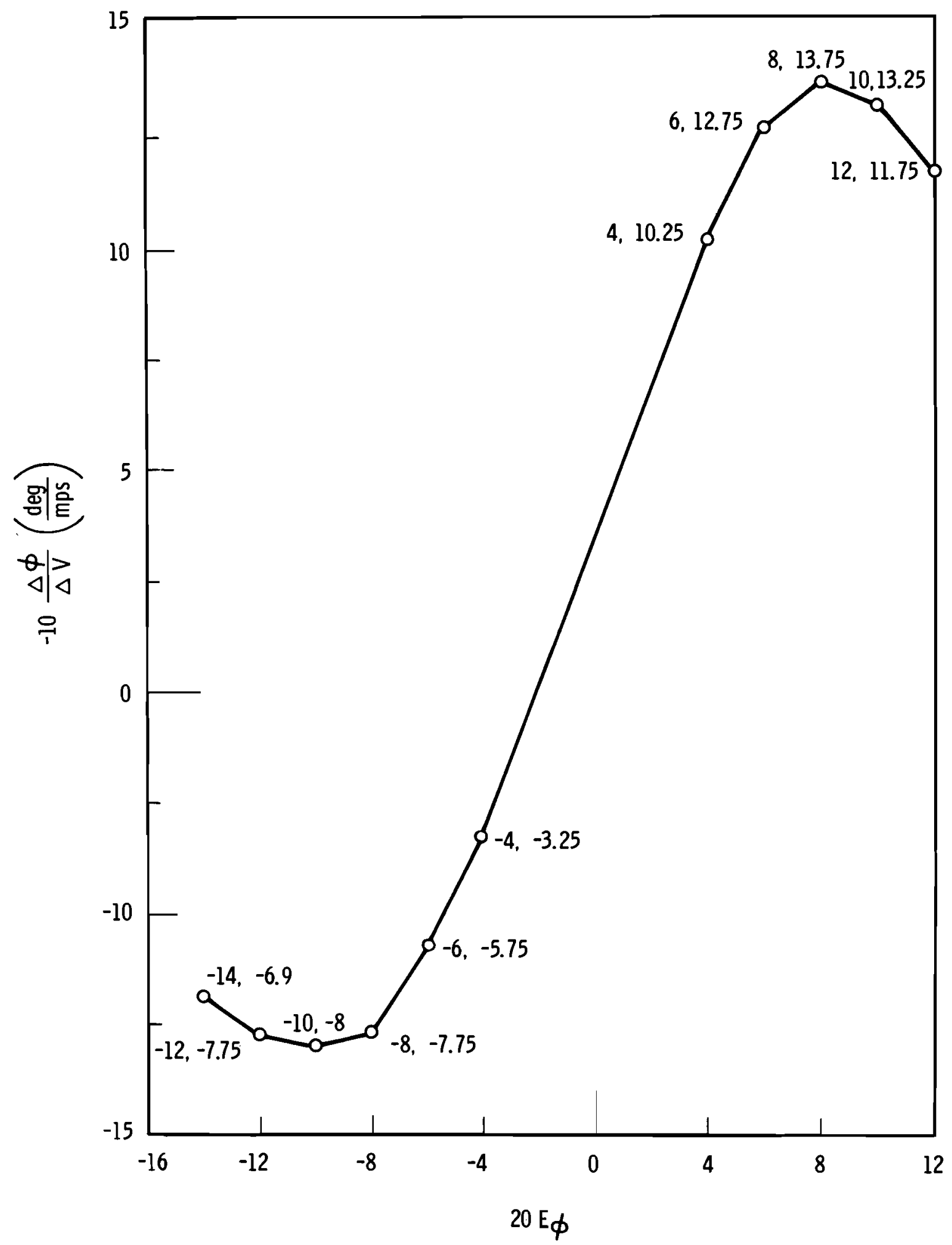

FIGURE 4.4 Function Generator Settings for Vertical Angle Correction 


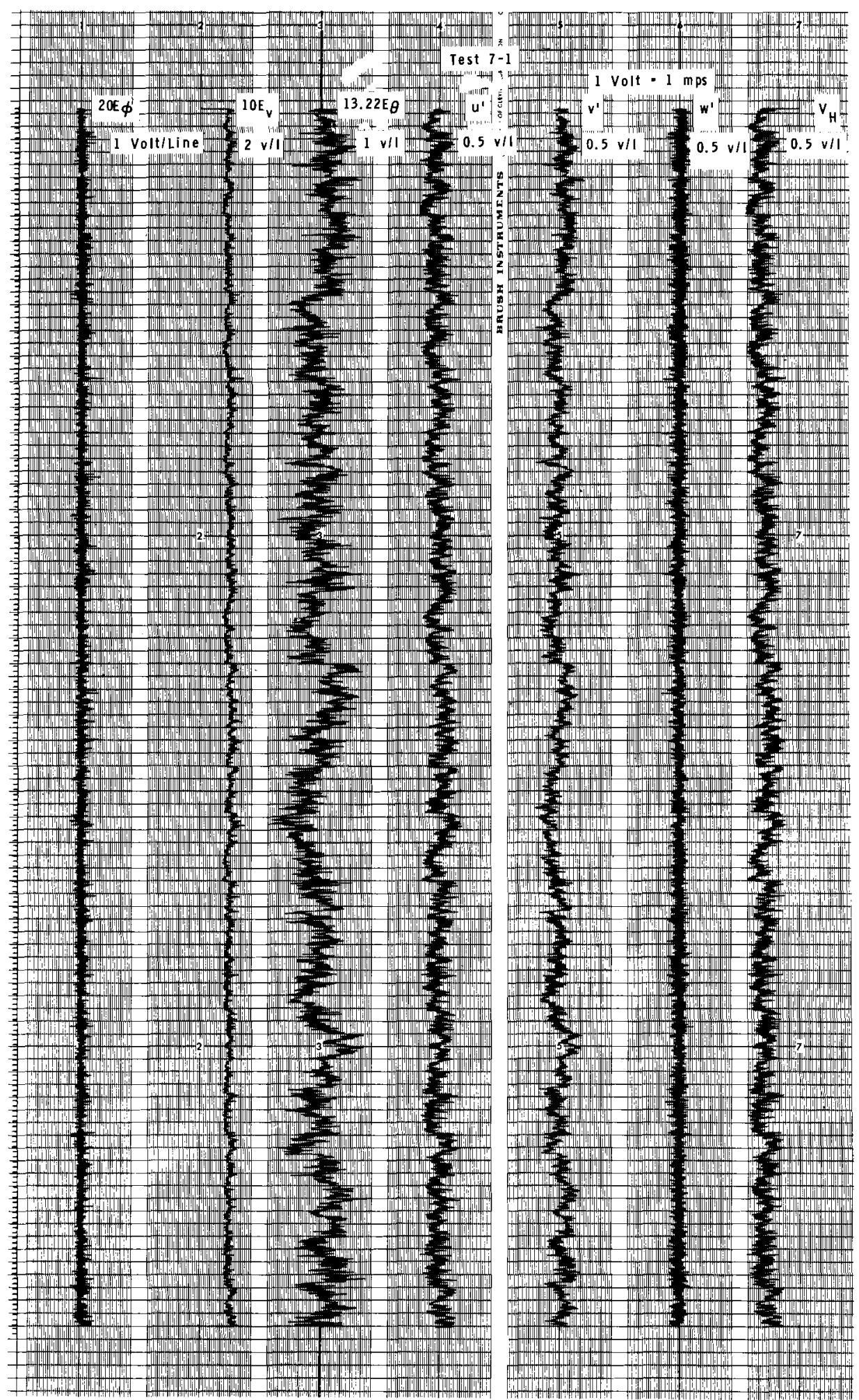

FIGURE 4.5 Coordinate Transformation Monitored Results 


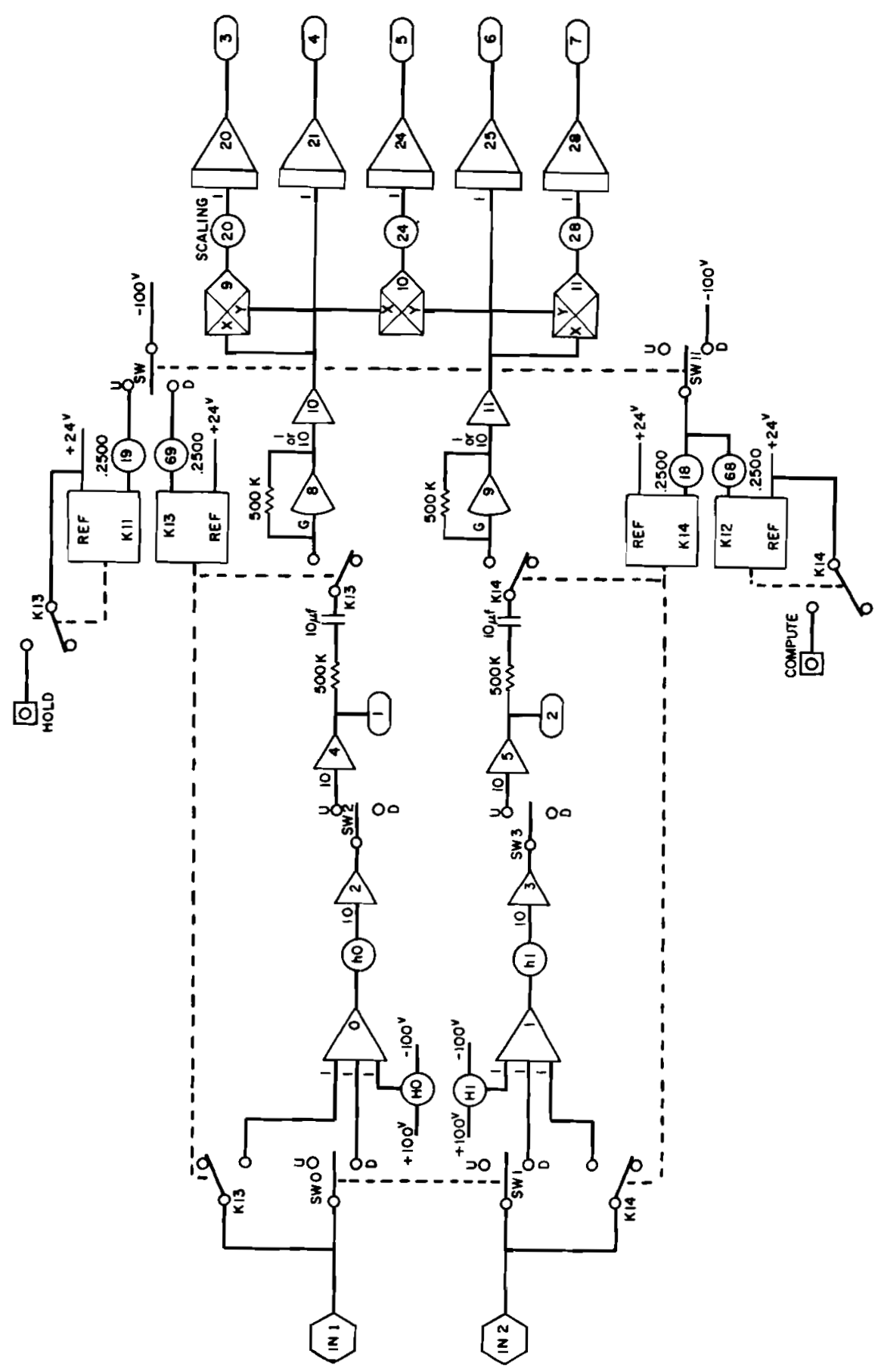

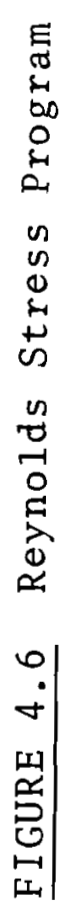




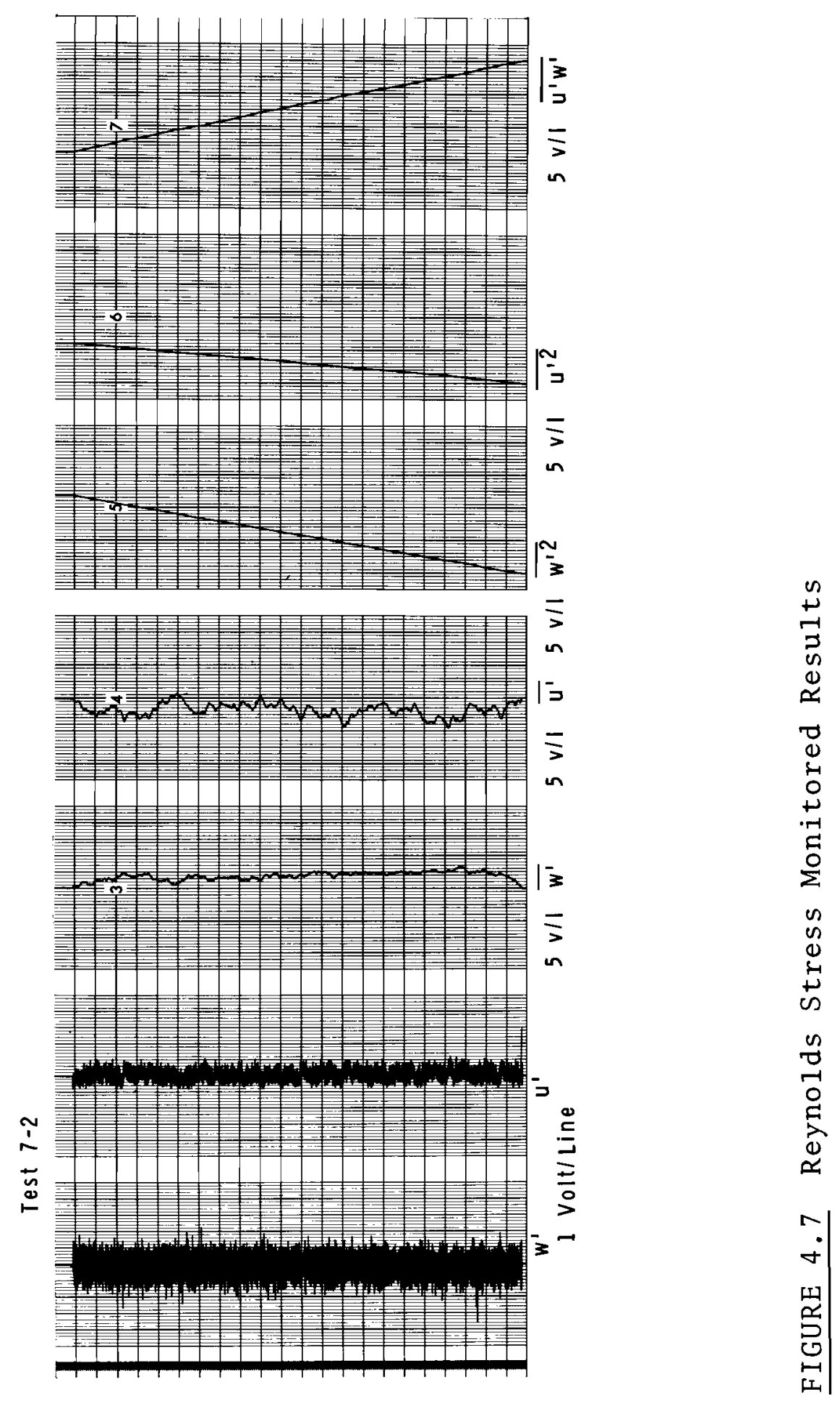




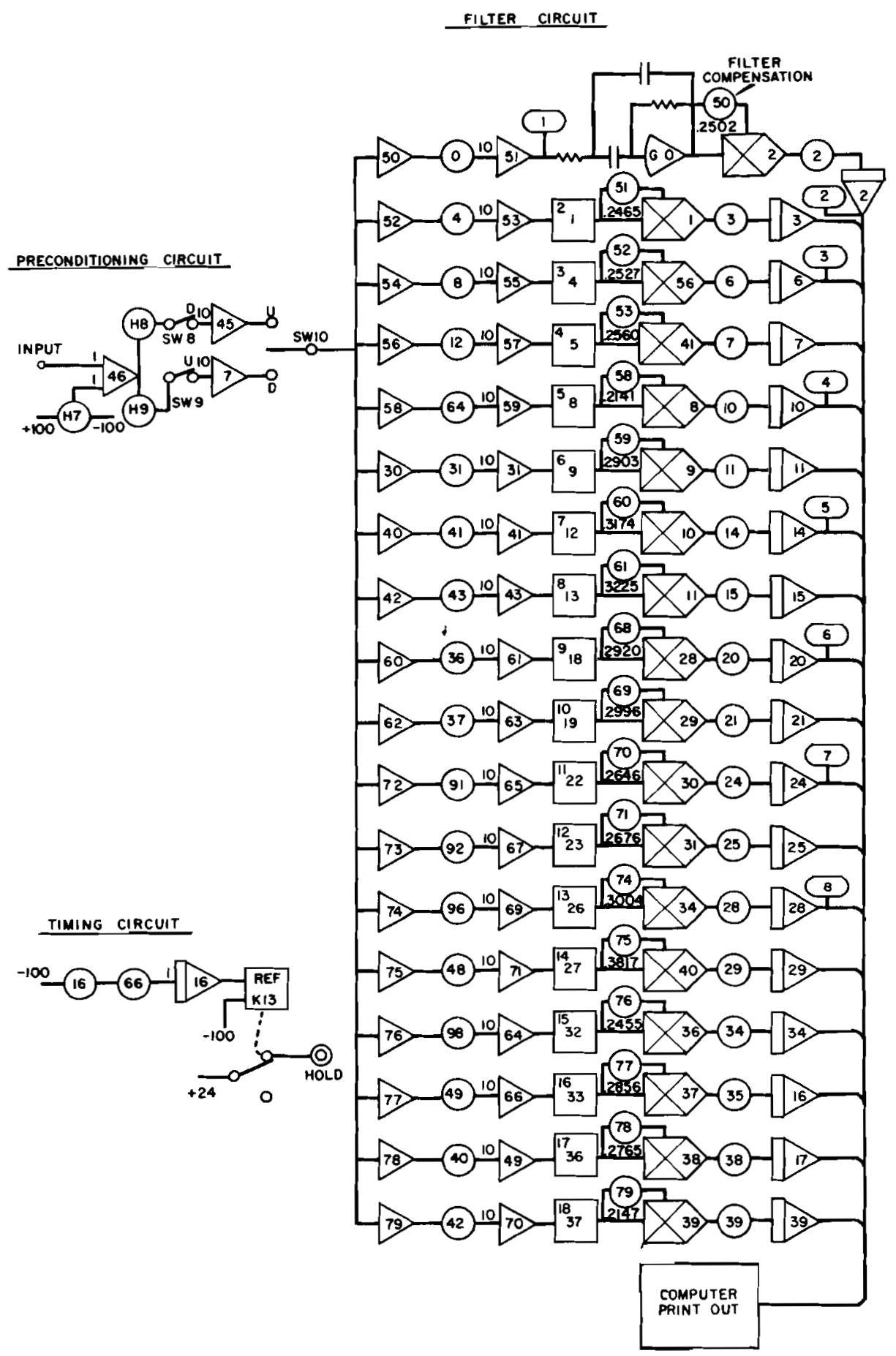

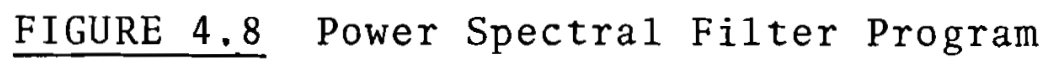


67

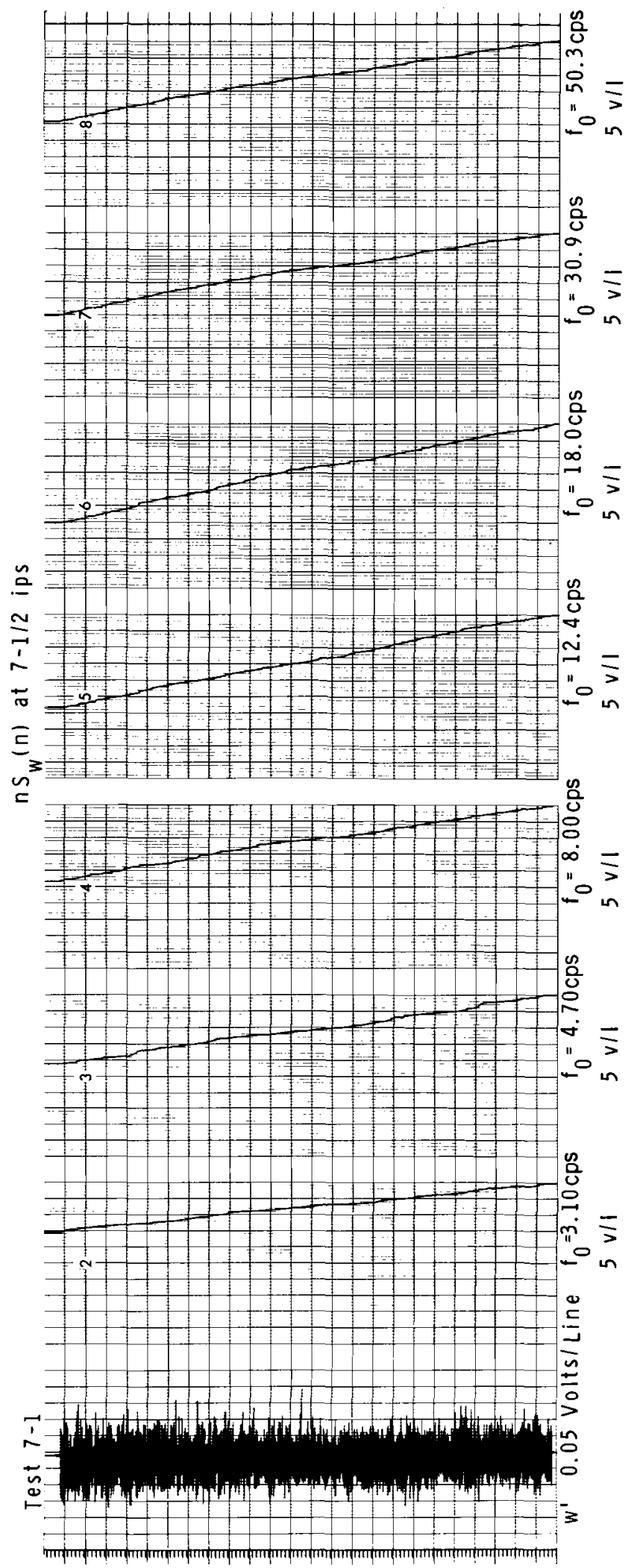

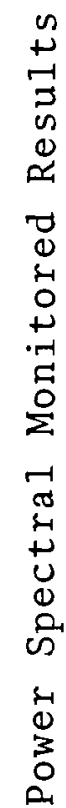

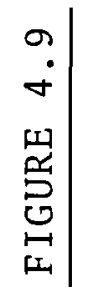


68

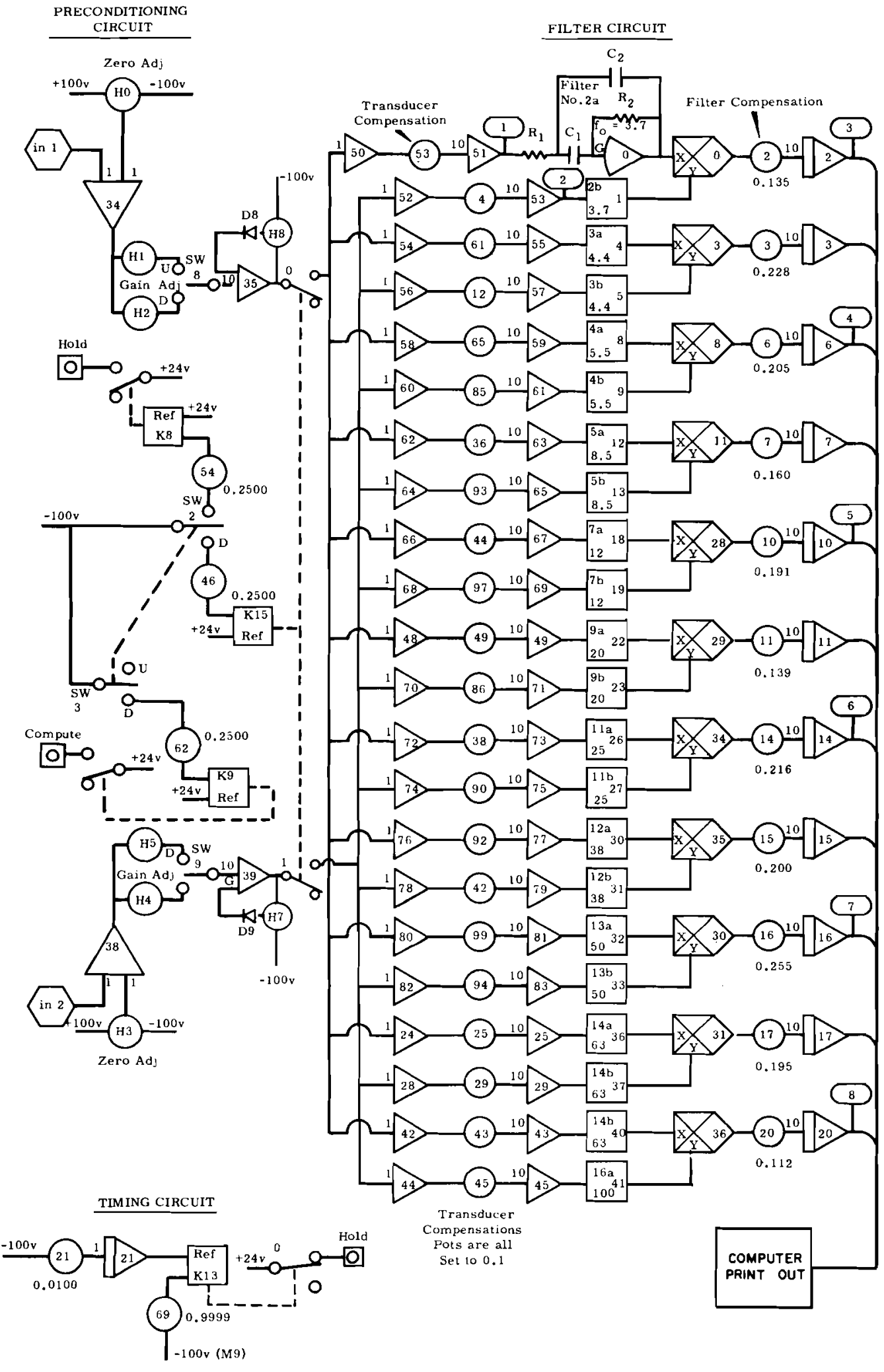

FIGURE 4.10 Conspectral Filter Program 
69

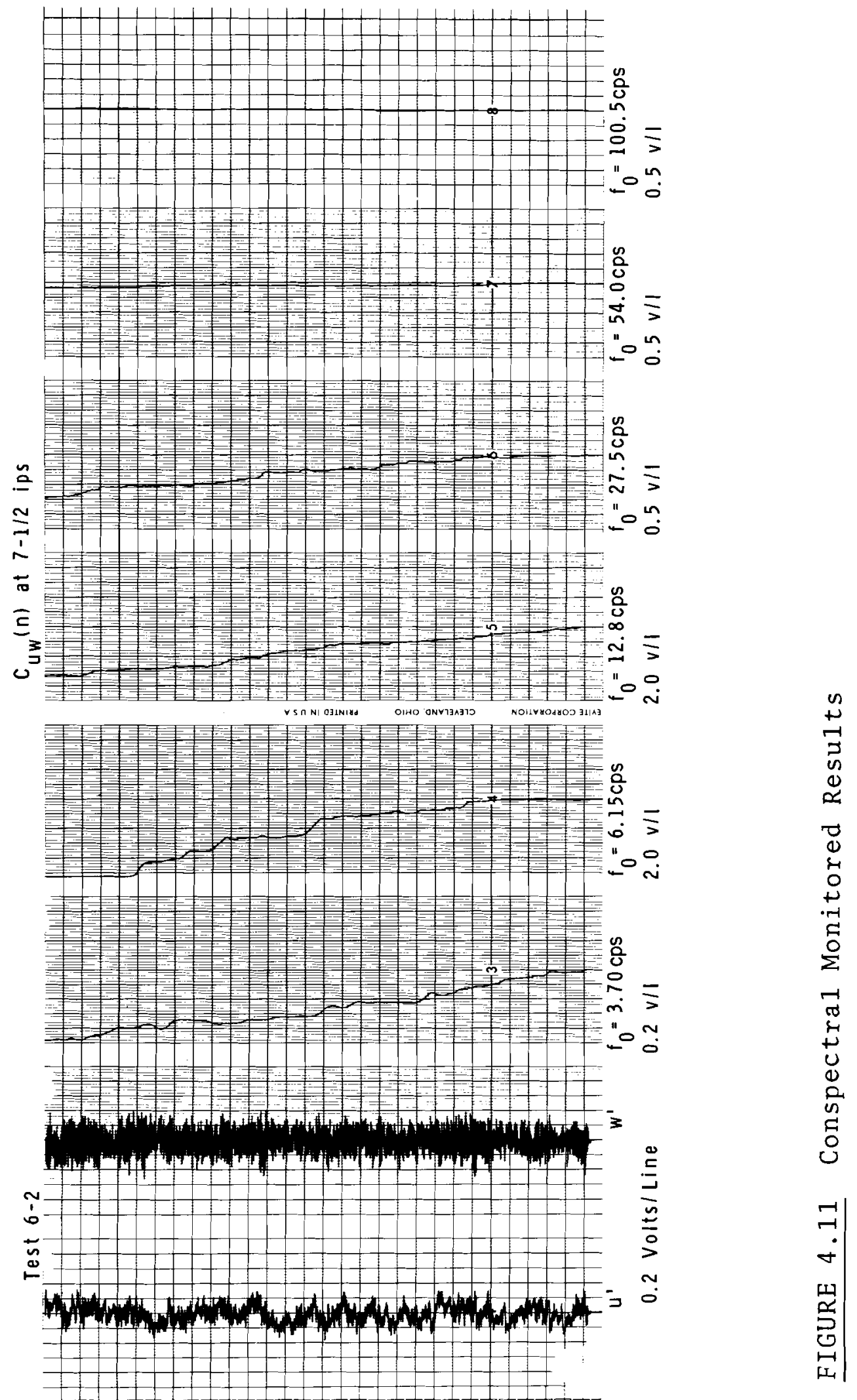




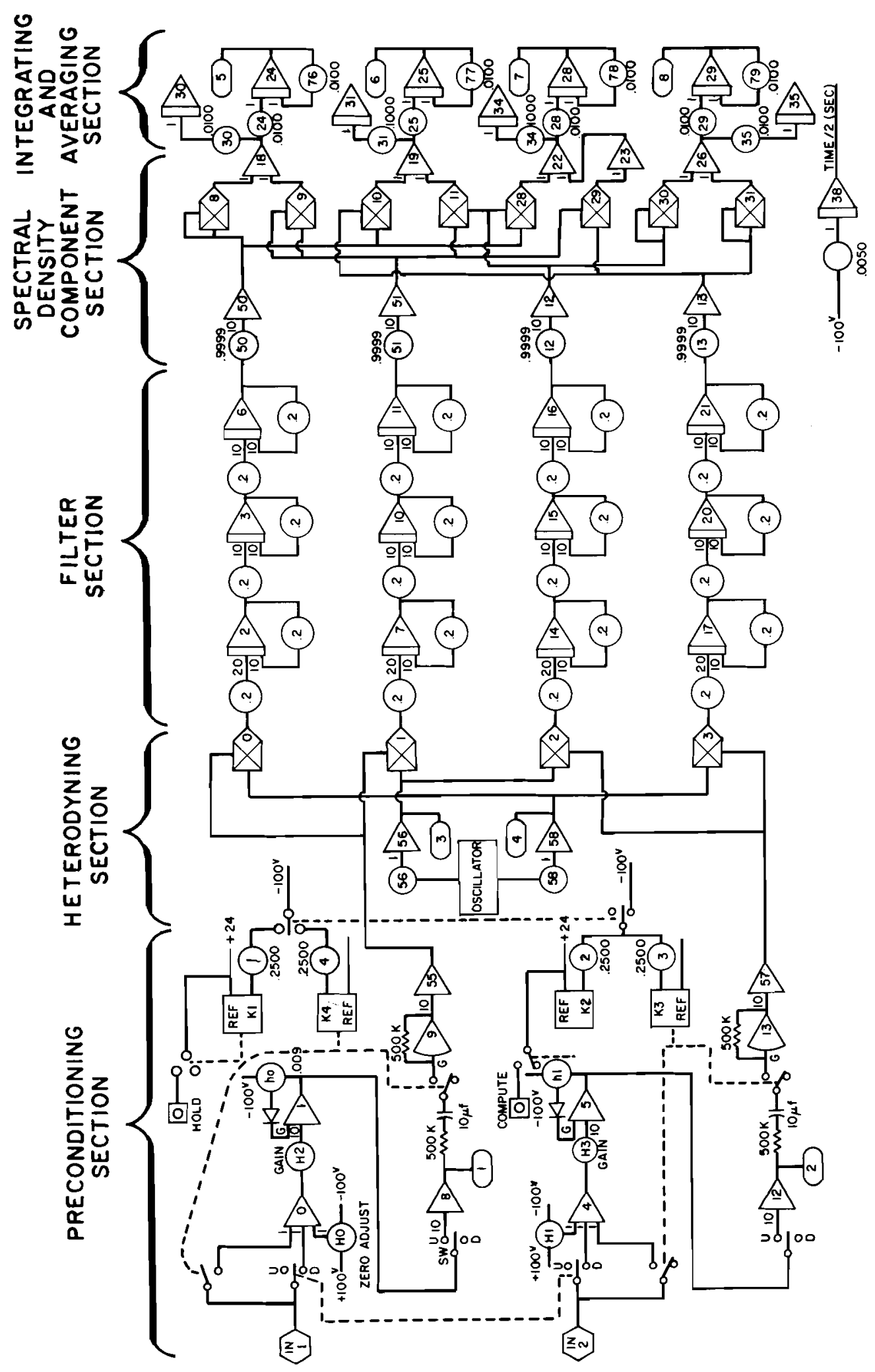

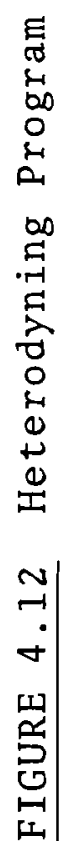




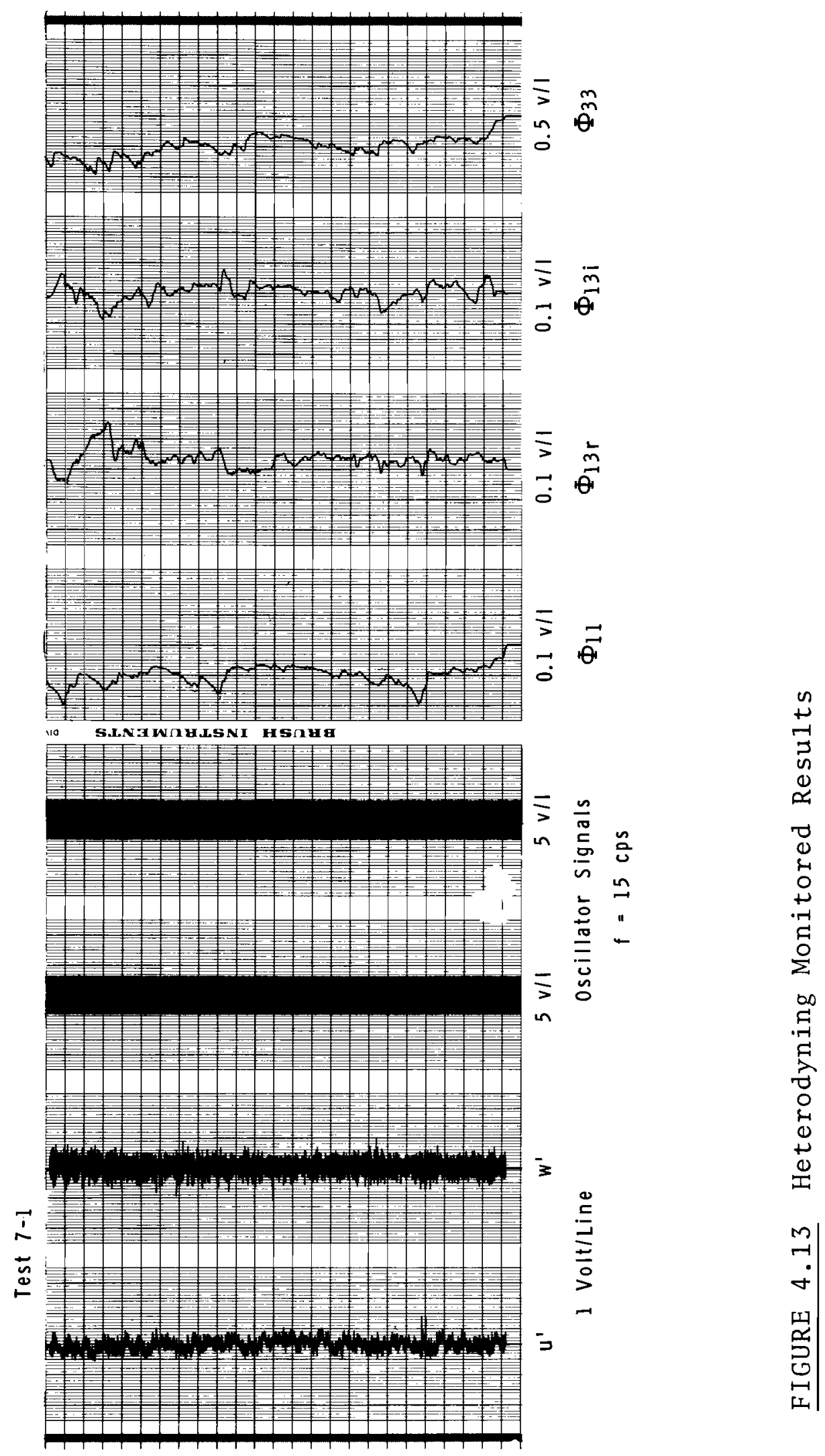




\section{FIELD TESTS}

In the summer of 1964 a series of turbulence measurements were conducted with the wind component meter mounted on a 24.4 meter meteorological tower called the Hanford Portable Mast at the 3.0 and the 6.1 meter levels. Measurements were made in neutral, unstable and stable conditions accompanied by a variety of wind speeds. Mean temperatures, wind speeds and directions were determined at logarithmic intervals from 0.8 meters to 24.4 meters on the tower with a system (Ratcliffe and Sheen ${ }^{40}$ ) utilizing copper-constantan thermocouples and Beckman and Whitley cup anemometers and wind vanes, sampling and logging the data automatically throughout the test series. The wind speeds and temperatures at 3.0 and 6.1 meters were consistently dependable and available during all but a few tests for Richardson's number calculations. The calculated gradient Richardson's number for the 4.3 meter level and the tower measurements of mean wind speed at the height of the turbulence measurements, are listed in Table 5.1. Also listed are the mean longitudinal wind component values calculated in the wind component meter data analysis which agree with the Portable Mast mean wind speed to within 9\%. The generally slightly larger values of mean wind speeds from the cup anemometers is to be expected since these represent the total horizontal component magnitude rather than the langitudinal component magnitude 
only. In addition, in this series of tests the wind component meter operation was not exactly synchronized with the mean value measurements so that data for slightly different periods of time were sampled by the two equipment systems, also contributing to the small discrepancies in the reported wind speeds.

Since data collected at greater heights were desired, later in the summer of 1964 the wind component meter was mounted at 12.2 meters where a single test in stable conditions was conducted. In the following spring, two additional tests were conducted at 12.2 meters and two more at 6.1 meters in neutral and unstable conditions. Also, the final test in unstable conditions was made with the wind component meter mounted at 87 meters on the Hanford 400foot meteorological tower. The accompanying meteorological data from the Portable Mast are again reported in Table 5.1 with the dates and times of the tests. For this series of tests the sampling periods for the wind component meter and the mean value system corresponded. However, the wind speeds reported for the wind component meter in Table 5.1 differ significantly from the wind speeds of the cup anemometer. The error was first suspected to be introduced in the analysis; however, reprocessing of one of the tests on the analog computer led to the same results. The only remaining possible explanation was that the speed calibration changed somewhat from the calibration made just prior 
to the first series of turbulence measurements. The calibration appeared to drift in one direction between the first series of tests and test 9-1, conducted a month later, then changed in the other direction during the intervening eight months before the final test series. The spectral estimates, normalized to total variance required no correction since the spectral estimates and the variances were both in error by the same factor. Whenever spectral estimates, variances or covariances are reported separately, the data for tests after the first series are corrected with the square of the ratio of wind component meter mean wind speed to tower measured wind speed. One correction factor was used for test 9-1 and another factor was used for the remaining tests during which time the calibration did not appear to drift further.

The turbulence data were recorded on the Ampex FR-1100 tape at 3-3/4 ips tape transport speed for all tests. Calibration signals of zero and one volt were recorded with the data to assure accurate reproduction of the data during the analysis period. The data were stored on the tapes until the analysis could be carried out a few months after the tests, with the analog computer programs discussed previously. 
TABLE 5.1

TEST CONDITIONS

Measurement
Height $\overline{\bar{U}} \quad{ }_{\bar{U}}^{W C M}$

\begin{tabular}{|c|c|c|c|c|c|c|c|}
\hline Test & Date & & ime & (meters) & (mps) & (mps) & $R i$ \\
\hline $2-1$ & $6-26-64$ & 0359 & -0430 & 3.0 & 5.29 & 5.16 & +0.01 \\
\hline $2-2$ & $6-26-64$ & 0512 & -0550 & 6.1 & 3.88 & 3.90 & +0.18 \\
\hline $2-3$ & $7-8-64$ & 0355 & -0430 & 3.0 & 4.10 & 3.77 & +0.05 \\
\hline $4-1$ & $7-16-64$ & 1930 & -1952 & 3.0 & & 2.18 & $\dagger$ \\
\hline $5-1$ & $7-17-64$ & 1647 & -1802 & 3.0 & 5.3 & 5.50 & -0.02 \\
\hline $5-2$ & $7-17-64$ & 1802 & -1824 & 3.0 & 6.3 & 6.54 & 0.00 \\
\hline $5-3$ & $7-17-64$ & 1840 & -1922 & 3.0 & 6.9 & 7.17 & 0.00 \\
\hline $6-1$ & $7-17-64$ & 2005 & -2018 & 3.0 & 5.4 & 5.23 & -0.02 \\
\hline $6-2$ & $7-17-64$ & 2018 & -2036 & 3.0 & 6.6 & 6.12 & -0.01 \\
\hline $6-3$ & $7-17-64$ & 2105 & -2207 & 6.1 & 8.1 & 7.40 & 0.00 \\
\hline $7-1$ & $7-18-64$ & 1410 & -1521 & 6.1 & 8.5 & 8.15 & -0.09 \\
\hline $7-2$ & $7-18-64$ & 1553 & -1655 & 3.0 & 9.0 & 8.59 & -0.04 \\
\hline $9-1$ & $8-11-64$ & 0140 & -0354 & 12.2 & 4.6 & 3.70 & +0.04 \\
\hline $11-1$ & $4-9-65$ & 1523 & -1629 & 6.1 & 3.6 & 5.27 & $-0.03+t$ \\
\hline $11-2$ & $4-19-65$ & 1134 & -1219 & 6.1 & 3.5 & 4.86 & -0.16 \\
\hline $12-1$ & $4-19-65$ & 1415 & -1520 & 12.2 & 2.1 & 3.20 & -0.10 \\
\hline $12-2$ & $4-28-65$ & 1507 & -1554 & 12.2 & 7.8 & 10.5 & -0.08 \\
\hline $13-1$ & $5-12-65$ & 1314 & -1414 & 87.0 & 7.8 & 11.1 & -0.07 \\
\hline
\end{tabular}

Hean wind and temperature data were not available during lest 4-1 but conditions were estimated to be slightly stable.

++ Evalu with smoothed data using all mast temperatures. 


\section{THEORETICAL BACKGROUND FOR TURBULENCE RESULTS}

Certain features of the measured spectra can be anticipated and used to organize spectral estimates. From Taylor's hypothesis 43 for a field of turbulence advected past a point of measurement, if the mean wind speed carrying the eddies is large compared to the turbulent velocity, the spatial distribution of turbulence is swept by essentially unchanged. Then the time dependent autocovariance function measured at a point can be expressed as a distance dependent function, $\phi(x)=\overline{u(t) u(t+x / \bar{v})}$ and its transform is

$\frac{\bar{U} S(n)}{2 \sqrt{2 \pi}}$, the turbulent energy density per unit wave member. Thus $\bar{U} S(n)$ plotted versus wave number, $n / \bar{U}$, should show the character of turbulence distributed in wave number space along the mean wind direction.

The scale of the turbulence is expected to be a function of height, the greater the height above the ground the larger are the eddies. In fact, the vertical size of the eddies is expected to be directly proportional to height as proposed by Monin 26 based on similarity theory considerations. Then plotting the spectra as functions of $\mathrm{nz} / \overline{\mathrm{U}}$ (the ratio of height to wave length) will tend to normalize the vertical component spectral distributions for comparison at all heights. Berman ${ }^{2}$ has organized the logitudinal spectra in the same manner, but found that they behaved differently at 
different heights opposed to the similarity hypothesis. since different levels of turbulence occur in different tests, the spectral estimates are normalized for comparison by dividing by the total variance, $\sigma_{u_{i}}{ }^{2}$. Also, since the total variance is expressed

$$
\begin{aligned}
\sigma_{u_{i}}{ }^{2} & =\int_{0}^{\infty} s_{u_{i}}(n) d n=\int_{0}^{\infty} n / \bar{U} \bar{U} s_{u_{i}}(n) \frac{d n z / \bar{U}}{n z / \bar{U}} \\
& =\int_{0}^{\infty} n / \bar{U} \bar{U} s_{u_{i}}(n) d(\ln n z / \bar{U})
\end{aligned}
$$

we can plot the product of wave number and normalized spectral density per unit wave number,

$$
\frac{n}{\bar{U}} \frac{\bar{U} s_{u_{i}}(n)}{\sigma_{u_{i}}{ }^{2}}=\frac{n s_{u_{i}}(n)}{\sigma u_{i}{ }^{2}} \quad \text { versus } \ln n z / \bar{U}
$$

and area under the curve between two wave numbers represents the fraction of the turbulent energy contributed by that range of wave numbers, the total area being unity.

The shape of the spectra must depend on the manner in which energy is introduced into and removed from the turbulence. The equation for the turbulent energy budget for steady state and horizontal homogeneity

$$
\begin{aligned}
& \frac{\partial e}{\partial t}=0=-\overline{u_{1} u_{3}} \frac{\partial \bar{U}}{\partial x_{3}}+\frac{g}{T_{0}} \overline{u_{3} \theta}-\overline{v \dot{\gamma} \frac{\partial u_{i}}{\partial x_{j}}\left[\frac{q u_{i}}{\partial x_{j}}+\frac{\partial u_{j}}{\partial x_{i}}\right]} \\
&-\frac{\partial}{\partial x_{3}} \overline{u_{3} e}-\frac{\partial}{\partial x_{i}} \overline{\left(u_{i} p_{p}\right)}
\end{aligned}
$$


describes the contribution and dispostion of turbulent energy by various mechanisms. Here we have assumed a mean wind in the $x_{1}$ direction, and stationary and homogeneous turbulence in the $x_{1}-x_{2}$ plane.

The first term on the riqht of Equation (6.2) is the rate at which energy is added to the turbulence in the $x_{1}$ direction taken from the mean motion through the working of the Reynolds' stress, $-\rho_{0} \overline{u_{1} u_{3}}$, aqainst the mean velocity graident, $\frac{\partial \overline{\mathrm{U}}}{\partial \mathrm{x}_{3}}$.

The second term describes the rate turbulent energy is introduced into or removed from the $x_{3}$ direction by the buoyancy force, resulting from density fluctiations, working in the gravitational field. The sign of the heat flux, $c_{p} \rho_{0} \overline{u_{3} \theta}$, determines whether turbulent energy is damped or amplified. The third term is the rate turbulent energy is dissipated into heat. The fourth term describes the transport of turbulent energy (as opposed to production or removal as in first three terms) resulting from the divergence of the flux of turbulent energy, $\overline{u_{3} e}$, where $e=1 / 2 \overline{\left(u_{i}\right)\left(u_{i}\right)}$. The last term is the summation of all those terms which account for the transfer of turbulent energy among components due to the correlation between the fluctuating pressure force and fluctuations in velocity.

The turbulent energy budqet equation indicates that energy is supplied to the turbulence from external influences 
only through the mechanical energy term and the convective energy term. Then, Monin and Oboukhov's similarity theory, assuming the turbulent regime is completely determined by the momentum flux, the heat flux and the buoyancy parameter, $g / \mathrm{T}_{0}$, predicts a dependence for the wind component standard deviations, $\sigma_{u}, \sigma_{v}$, and $\sigma_{w}$ on the friction velocity and $a$ universal function of dimensionless height, $z / L$ (Monin 25 ). Similarly Panofsky and McCormick ${ }^{4}$ following the similarity theory argument have tested a relationship for the standard deviation of vertical velocity, proportional to the friction velocity and a universal function of the dimensionless height $2 / L$, i.e.

$$
\sigma_{W}=B u^{\star} h(z / L)
$$

where $B$ is a universal constant, I is Monin's length,

$$
\mathrm{L}=-\mathrm{u}^{\star 3} \mathrm{~T}_{0} \mathrm{c}_{\mathrm{p}} \rho \delta / \mathrm{kgH}, \mathrm{u}^{\star}=\sqrt{-\overline{\mathrm{uw}}}
$$

is the friction velocity and $H=c_{p}$ of $\overline{w \theta}$ is the turbulent heat flux. Since $z / L$ is uniquely related to the Richardson number, we can look for the standard deviations of the velocity component fluctuations to be proportional to friction velocity and a function of the Richardson number. In neutral conditions the standard deviations should simply be proportional to friction velocity. Similarity theory likewise predicts the dimensionless wind shear, $\phi=\frac{\partial \bar{U}}{\partial z} / \frac{u^{*}}{\mathrm{kz}^{*}}$, the ratio of wind shear in diabatic conditions to that in 
neutral conditions, to be a universal function of $z / L$ or Richardson's number.

The ranges of wave numbers over which the terms of Equation (6.2) are effective will, in part, determine the shapes of the spectra as well as does the manner in which the energy is transferred (primarily due to vortex stretching) from low wave numbers where it is generally introduced, to high wave numbers where it is removed by dissipation. Dissipation acts principally at high wave numbers where velocity gradients are very large though fluctuation magnitudes are very small. It is expected this will occur beyond the range of wave numbers detectable with instrumentation used in the present experimental investiqation. Lumley and Panofsky 20 cite measurements between 1 and 100 meters which indicate the Kolmogoroff microscale, the wave length where viscosity becomes dominant, is of the order of $1 \mathrm{~mm}$. It is expected, further, that near the around surface in the range of wave numbers where energy is introduced into the spectra, the rate of production of mechanical turbulent energy outweighs the production rate of convective turbulent energy. This occurs because the gradient of velocity is large near the surface and decreases upward while the Reynolds' stress remains constant so the mechanical energy term decreases upward from a large value near the surface. Thus, in the turbulent layer closest to the surface the mechanical energy or shear production term should dominate 
the structure of the turbulence in the energy production range of eddy sizes, even in somewhat diabatic conditions, while the buoyancy term should have a relatively imperceptible effect. The heat flux, and consequently the buoyancy term, remains constant with height. Therefore, at qreater heights where velocity gradients become small, the buoyancy term should contribute most significantly to the turbulent energy. This contribution may occur relatively independent of the mechanical energy contribution and at lower wave numbers. Also, Lumley and Panofsky 20 suggest the scaling of the convective eddies by the height and the wind speed should not hold but should be determined by the thickness of the unstable layer and the character of the surface inhomogeneities.

In unstable conditions, the vertical turbulent transfer of heat is often considered occurring as a result either of forced convection or of free convection. Priestley 39 describes forced convection as the condition when the buoyancy does not significantly affect the motion or the heat transfer coefficient but the mechanically generated turbulence is responsible for the vertical flux of heat as well as momentum. Through the similarity theory the wind profile is shown to be a logarithmic plus a linear function of height and the temperature gradient is similarly loglinear. Near the ground, however, the profiles often cannot be experimentally distinguished from the simple logarithmic 
neutral profiles. Free convection occurs when the air motion responsible for vertical fluxes result from buoyancy, i.e., the low frequency buoyant eddies, highly efficient in transferring heat, are primarily responsible for the heat flux and the momentum transfer as well. Therefore, a region of forced convection will often exist in the atmosphere with a region of free convection above. The sharpness of the transition from forced to free convection is open to question but characteristics of each should be observed in the spectra.

Also, when the buoyancy term is significant the transport term $\frac{\partial}{\partial x_{3}} \overrightarrow{u_{3} e}$ may be of importance. A few measurements in unstable conditions, discussed by Panofsky 30 have indicated that in unstable conditions the upward flux of turbulent energy increases with height so that the flux divergence tends to compensate for the addition of convective energy from the working of the density fluctuations. This term is seen to be effective only in transporting energy from one place to another since it is a divergence and its integral over a large enough volume can be restated through the divergence theorem as a surface integral through which no transport occurs. Thus there is no net loss or qain of energy from the flux divergence term.

Little is known of the ranges of wave numbers over which the term describing the work done by the fluctuating pressure gradient is effective. Expressing this term: 
$\overline{\frac{u_{i}}{\rho_{0}} \frac{\partial p}{\partial x_{i}}}=\frac{1}{\rho_{0}}\left[\frac{\partial}{\partial x_{i}} \overline{\left(u_{i} p\right)}-\overline{p \frac{\partial u_{i}}{\partial x_{i}}}\right]$

and integrating over a volume larqe enough to include all eddy sizes,

$$
\iiint \frac{u_{i}}{\rho_{0}} \frac{\partial p}{\partial x_{i}} d v=\frac{1}{\rho_{0}}\left[\iiint \frac{\partial}{\partial x_{i}} \overline{\left(u_{i} p\right)} d v-\iiint \frac{\partial u_{i}}{\rho \frac{\partial x_{i}}{x}} d v\right]
$$

For the first term on the right we have the integral of a divergence re-expressible throuqh the diverqence theorem as a surface integral of a flux. Thus the first term is zero considering horizontally homogeneous conditions and no flux of the quantity, $\overline{u_{i} p}$, through the upper and lower surfaces of the boundary layer. The second term is also zero from the equation of continuity so there is no net work done by the fluctuating pressure gradient but enerqy can be transferred from one region within the volume to another. Also, we can now expand this term, giving

$$
\iiint \frac{\overline{u_{1}}}{\rho_{0}} \frac{\partial p}{\partial x_{1}} d v=-\iiint \frac{\overline{u_{2}}}{\rho_{0}} \frac{\partial p}{\partial x_{2}} d v-\iiint \frac{\overline{u_{3}}}{\rho_{0}} \frac{\partial p}{\partial x_{3}} d v
$$

Thus the total power associated with the work done by the fluctuating longitudinal wind component against the fluctuating pressure gradient in the same direction equals the work done on the air moving with the other components by their 
respective pressure gradients. In this way, turbulent enerqy is transferred out of the $x_{1}$ component into the $x_{2}$ and $x_{3}$ components. This transfer might appear in one component over a given range of wave numbers while not in another. In view of the limitations placed on both the size of the vertical eddies and the scale of the vertical pressure gradient fluctuations by the proximity of the surface, it might be anticipated that the turbulent enerqy generated in the $x_{1}$ direction through the Reynolds' stress term would be transferred to the vertical component over a relatively high wave number range. The energy transferrer into the $\mathrm{x}_{2}$ direction, however, is not subject to such constraints and might occur over any part of the cospectral wave number range.

The action of pressure forces, transferring turbulent energy among components, leads to equipartition of energy at higher wave number, and as the cospectra for each pair of components decreases at increasing wave numbers, eventually the eddies approach isotropy over the upper end of the power spectrum.

The turbulent fluctuations associated with these eddy sizes were defined as locally isotropic by A. Kolmogoroffl. $\mathrm{His}$ definition required steadiness of the turbulence in time but dealt only with differences in velocity, from one point to another, the probability distribution for the velocity differences between all points within a given space 
and time domain being invariant with translation, rotation or reflection of the coordinate axes, allowing a description of isotropic eddies existing in a field of turbulence along with anisotropic eddies. Kolmogoroff then hypothesized that the probability distribution of the velocity differences or the average properties of the turbulence for locally isotropic turbulence was uniquely determined by the rate of dissipation of energy, $\varepsilon$, and the kinematic viscosity, $v$. He considered that below the isotropic range energy is fed from the large anistropic eddies, is passed from lower to higher wave numbers by inertial forces and is eventually dissipated by viscous forces at the same rate it is fed into the isotropic range, matntaining an equilibrium state. Kolmogoroff hypothesized further that the equilibrium range of eddy sizes through which the energy is passed might have at its lower wave number end, a range of eddy sizes where viscous forces are not effective so no dissipation takes place. Thus, the average properties of the turbulence in this "inertial subrange" are determined only by $\varepsilon$, the rate at which energy is inertially fed through the subrange and eventually dissipated beyond it. Then by dimensional reasoning the variance of the velocity difference or the structure function, which is a function only of $\varepsilon$ and the separation, $x$, must be proportional to $(x)^{2 / 3}$. Then taking the Fourier transform, 


$$
S(k) \propto \varepsilon^{2 / 3} k^{-5 / 3}
$$

which is often called Kolmogoroff's minus five-thirds law for the three-dimensional spectral density in the inertial subrange. We can also write

$$
n S(n)=K S(k)=A \varepsilon^{2 / 3} k^{-2 / 3}
$$

The one-dimensional spectrum has the form

$$
n S(n)=k_{1} S\left(k_{1}\right)=a \varepsilon^{2 / 3} k_{1}^{-2 / 3}
$$

where "a" is a constant which, indicated by the most recent determinations, is between 0.45 and 0.50 if $k$ is given in radians per unit length. Lumley and Panofsky ${ }^{20}$ suggest using a value of 0.47 for the longitudinal velocity spectral relationship. Henceforth, wave number will be expressed in cycles per meter for which the suggested value of the constant, a, is 0.138 .

The preceding expression is for the distribution of the variance in $u_{1}$ among wave numbers, $k_{1}$, where the subscript, 1, refers to the axes directed along the mean flow. The distribution of the variance in $u_{2}$ or in $u_{3}$ among wave numbers $\kappa_{1}$ can be shown (Kolmogoroff ${ }^{17}$ ) to be identical in form but multiplied by $4 / 3$; that is, the constant appearing in the expression is $4 / 3 a$. The line of reasoning on which the proof of this relationship depends is that the turbulence be isotropic at the wave numbers concerned and that the 
equation of continuity for an incompressible fluid be valid. In the remainder of this presentation only the spectral distributions in the one-dimensional wave number space, $k_{1}$, will be considered. Henceforth, $k_{l}$ will simply be indicated by $k$ or by its estimate, $n / \overline{\mathrm{U}}$. Furthermore, as has already been done on a few occasions, the notation for the directions $x_{1}, x_{2}$, and $x_{3}$ will be replaced with $x_{,} y$, and $z$ respectively and the corresponding wind component fluctuations $u_{1}, u_{2}$, and $u_{3}$ by $u^{\prime}, v^{\prime}$ and $w^{\prime}$. Also, $u^{\star 2}$ will often be used for the Reynolds' term, $-\overline{u_{1} u_{3}}$.

Then a further expectation would be that a region of the measured spectra, above the energy adding range at low wave numbers, will follow the relationship for the inertial subrange. The level of turbulence in the inertial subrange is set by the rate of dissipation which, for neutral conditions, can be determined from the mechanical energy production term. This can be seen from Equation (6.2) where no other means of production or removal of turbulent energy except from the first and third terms is significant for these conditions. Then it follows that Equation (6.2) becomes

$$
\varepsilon=u^{\star 2} \frac{\partial \bar{U}}{\partial z}=u \star 3 / k z
$$

in view of the logarithmic profile.

When this relationship holds, we can see that the inertial subrange is scaled according to height since Equation 
(6.3) becomes

$$
\begin{aligned}
\mathrm{nS}_{u}(n)=a \varepsilon^{2 / 3} k^{-2 / 3} & =a(u \star 3 / k z)^{2 / 3}(\mathrm{n} / \overline{\mathrm{U}})-2 / 3 \\
& =a u^{\star 2} \mathrm{k}^{-2 / 3}(\mathrm{nz} / \overline{\mathrm{U}})-2 / 3
\end{aligned}
$$

and if the spectrum is normalized to the square of the friction velocity, a universal function should be expected for the inertial subrange in neutral conditions:

$$
n S_{u}(n) / u * 2=a k^{-2 / 3(n z / \bar{U})}-2 / 3
$$

where, of course, $a \mathrm{k}^{-2 / 3}$ is a universal constant. For the transverse component spectra the additional factor of $4 / 3$ is required. Furthermore, if another universal constant relates the total variance to the friction velocity, then we can write

$$
n \mathrm{u}_{\mathrm{u}}(\mathrm{n}) /_{\mathrm{u}}^{\mathrm{\sigma}}{ }^{2}=\mathrm{a} / \mathrm{C}^{2} \mathrm{k}^{2 / 3}(\mathrm{nz} / \overline{\mathrm{U}})^{-2 / 3}
$$

Again for the transverse spectra, the $4 / 3$ factor is involved and the appropriate constant relating variance to friction velocity must be used.

Berman $^{2}$ alters the relationship $(6.3 a)$ to account for diabatic conditions giving

$$
n \mathfrak{q}(n) / u^{\star^{2}}=a k^{-2 / 3}(n z / \bar{U})^{-2 / 3} g(R i)
$$

Here, the dissipation is aqain set equal to the mechanical energy production, assuming the buoyancy term in the 
turbulent energy budget equation is balanced by the flux divergence term. However, it is necessary to account for the deviation from the logarithmic profile for diabatic conditions in the mechanical energy production term. This is accomplished by including the factor $\mathscr{\&}(R i)$, the ratio of the true wind speed shear to the shear for a loqarithmic profile, a function of the stability as indicated by Richardson's number, Ri. Close to the ground even in diabatic conditions, since the mechanical energy production usually predominates, the lower portion of the wind profile is quite close to logarithmic and Equations (6.3a) and (6.3b) can be used in the forced convection region. Only a small error results, on the order of that introduced in the measurement and analysis of the turbulence data.

The observations made with the wind component meter have been subjected to analysis in the framework of the above discussion. In evaluating the results it is well to bear in mind that some details of the theory are not firmly established. In particular, any theoretical development depending on isotropy is open to question. 
VII. RESULTS OF ANALYSIS

\section{Longitudinal Component Spectra}

The spectra for the longitudinal component were plotted in the manner suggested by the discussion of the previous section, with the normalized spectral density, $n s_{u}(n) / \sigma_{u}{ }^{2}$ on the ordinate and $n z / \bar{U}$, the ratio of height to wave lenqth, plotted logarithmically on the abscissa. The tests were grouped according to stability, Fiqure 7.1 showing the spectra for neutral conditions, Figure 7.2 for unstable conditions and Figure 7.3 for the stable cases. In addition, the cases above 12 meters, where buoyancy effects are expected to be most in evidence, were plotted separately in Figure 7.4 .

The similarities of the spectra, plotted in this manner, are to be noted. Differences are observed between stability groups, however. For all stabilities, peaks in the spectra reached maximum normalized values near 0.12 and were found at an $n z / \bar{U}$ value of about 0.01 to 0.04 . For stable cases an additional peak was found near $n \approx / \bar{U}=0.1$. The stable data were shifted to slightly higher wave numbers than the data for nertral and unstable conditions. One test, 9-1, in stable conditions at 12.2 meters conducted above the region dominated by the mechanical energy production, demonstrates a marked deviation from the form exhibited by the other data. The spectra for both the horizontal and vertical components for this single test were 
shifted to much higher $n z / \vec{U}$. This is to be expected since at greater heights where buoyancy effects dominate, the height above the surface should no longer determine the scale of the turbulence for the stable case but some stability length, small compared with $z$ such as Monin and Oboukhov's parameter, L, would control the size of the eddies.

The energy adding region of the longitudinal wind component spectra corresponds quite well with the region of maxima for the $\overline{u^{\prime} w^{\prime}}$ cospectra, responsible for the feeding of energy into the longitudinal turbulence from the mean motion. This can be seen by comparing the spectral distributions of Figures $7.1,7.2,7.3$ with the cospectral distributions of Figures $7.25,7.26,7.27$, and 7.28 .

The spectra for the longitudinal component are also shown with the logarithm of $n S_{u}(n) / \sigma_{u}{ }^{2}$ plotted versus the logarithm of $\mathrm{nz} / \overline{\mathrm{U}}$ in Figures 7.5 through 7.8. The universal form of Equation (6.3b) for the inertial subrange is also shown in Figures 7.5 and 7.6 and reasonably good verification of the inertial subrange is observed for neutral conditions, Equation (6.3b) appearing about 208 low when the value $\mathrm{a}=0.138$ is used. Better agreement is seen with the unstable data. For both cases only $\sigma_{u}{ }^{2} / u^{\star 2}$ ratios for tests shown in each figure were averaged to obtain the curves representing Equation (6.3b). In Figure 7.8 the observed correspondence of the unstable cases above the forced convection region with those within, throughout the 
inertial subrange, could not have been anticipated from Equation $(6.3 \mathrm{~b})$. It is particularly surprising to find reasonable correspondence over most of the spectrum for the test at 87 meters where essentially no correlation exists between the vertical and longitudinal components and where the momentum flux is apparently accomplished through the large $\overline{v^{\prime} w^{\prime}}$ Reynolds stress.

For stable longitudinal component spectra the lower end of the inertial subrange would appear to be at about $\mathrm{nz} / \overline{\mathrm{U}}=1.0$ while for neutral and unstable conditions the minus five-thirds law seems to extend to considerably lower wave numbers, about 0.2 to 0.4 . However, the cospectra for all pairs of components, shown in Fiqures 7.25 through 7.37 , show the requirements for isotropic conditions (that the correlation between all pairs of components must be zero) generally hold only at greater wave numbers than $n z / \bar{U}=1.0$ to 3.0 .

The outstanding example of this is the stable test at 12.2 meters where the power spectrum is generally displaced to higher wave numbers but the minus five-thirds law extends to the peak at $n z / \bar{U}=0.2$, whereas the cospectra confirm isotropic conditions only above 4.0 .

Vertical Component Spectra

The spectra for the vertical component are shown in Figures 7.9 through 7.16 where the normalized spectral 
density, $\mathrm{nS}_{\mathrm{W}}(\mathrm{n}) / \sigma_{\mathrm{W}}{ }^{2}$, is plotted versus the logarithm of $\mathrm{nz} / \overline{\mathrm{U}}$ in Figures 7.9 through 7.12 and the loqarithm of each is plotted in Figures 7.13 through 7.16. The universal character of the turbulence for this component can be seen with only a few exceptions, again for measurements above the forced convection region shown in Figures 7.12 and 7.16 . The maxima for all vertical component spectra occur at a considerably hiqher value of $n a / \bar{U}$ than for the longitudinal component, a possibility considered earlier, appearing generally at about $n z / \bar{U}=0.4$ with the peak normalized spectral estimates averaging about 0.2 . These values and the same spectral shape hold within the forced convection region for neutral and unstable conditions with a suqgestion of a slight shift to higher wave numbers for stable cases, below 12 meters. The lower limit for the minus five-thirds law occurs at about $n z / \bar{U}=1.2$ for the stable cases; for neutral and unstable conditions it extends to about 0.8 or 1.0 . As mentioned previously, the lack of correlation between components above $n z / \bar{U}=1.0$ to 3.0 for neutral and unstable conditions confirms the isotropic condition necessary for the inertial subrange at slightly higher wave numbers than those over which the minus five-thirds law was observed in the logarithmic spectral plots.

Again, Equation $(6.3 b)$ is shown for neutral and unstable conditions in Figures 7.13 and 7.14 where only cases shown in each figure were used to determine the average values 
of the ratio $\sigma_{w}^{2} / u^{\star 2}$. Reasonably good agreement is again obtained using the value $a=0.138$ and in this case the additional factor of $4 / 3$.

Spectral peaks in unstable conditions within the forced convection region resulting from the direct introduction of convective turbulent energy into the vertical component are notably absent. Only for test $11-2$, at 6.1 meters, were there a few large spectral estimates at low wave numbers suggesting convective energy. This is contrasted in Figure 7.16 with the definite convective peak for test 12-2, an unstable case at 12.2 meters, noted in the $\mathrm{nz} / \overline{\mathrm{U}}$ range below 0.06 . The unstable test at 87 meters 1 ikewise indicates convective energy with the lowest two spectral estimates at about $n=/ \bar{U}=0.15$. Test $12-1$, an unstable case for the 12.2 meter height also shows a peak, similar to the other convective peaks, at a lower wave number than that introduced by the mechanical turbulence, in this case at $\mathrm{nz} / \overline{\mathrm{U}}=0.13$. This test, though conducted under cover of a heavy overcast, was carried out on a summer day with a very light wind. The resulting total vertical velocity variance was so small that even the slight convective energy added, corresponding to a very small temperature difference, was noticeable when compared with the very small amount of mechanical energy, as seen in the spectrum. As expected, the convective peaks do not appear to scale according to height. In terms of frequency, the 12.2 meter unstable case has 
convective energy through the lower end of the sampled spectrum, below 2.3 cycles/minute. The 87 meter unstable case has convective energy apparent below 0.9 cycles/minute and the light wind speed case at 12.2 meters shows convective energy below 1.4 cycles/minute. Again, the correspondence of the spectra for unstable cases above with those within the forced convection region, throughout the inertial subrange, in spite of the inapplicability of Equation (6.3b) should be mentioned. Also to be noted is the occurrence of a "mechanical energy production peak" for all these cases at about the same $n z / \bar{U}$ value as observed within the forced convection region.

The vertical component spectrum for the test in stable conditions at 12.2 meters was found, as with the longitudinal component, to be displaced to higher wave numbers as shown in Figure 7.16. The inertial subrange is seen from spectral and cospectral distributions to begin at about $n z / \bar{U}=4.0$.

\section{Lateral Component Spectra}

The lateral component spectra are not as well organized as the vertical and longitudinal spectra. A large fraction of the total variance appears at low wave numbers in a disorganized manner as a function of $\mathrm{nz} / \overline{\mathrm{U}}$ and with widely varying magnitudes, for neutral and unstable conditions. However, a degree of similarity is detected in the lateral spectra shown in Figures 7.17 through 7.24. All spectra generally reduce in amplitude with increasing wave 
number above about $n z / \overline{\mathrm{U}}=0.1$.

For neutral conditions, as seen in Fiqure 7.17 , multiple peaks at wave numbers below $n z / \bar{U}=0.1$ are common. For unstable conditions a range of even more consistently high spectral estimates below about $n z / \bar{U}=0.1$ is observed in Figure 7.18. The stable tests demonstrate the most consistent similarity, seen in Figure 7.19 , exhibiting a marked absence of the energy at low wave numbers found in the other stability groups. The stable group is again shifted to somewhat higher wave numbers than the neutral and unstable cases and the 12.2 meter stable test in Fiqure 7.20 is displaced to considerably higher $\mathrm{nz} / \overline{\mathrm{U}}$.

The $v^{\prime}$ component spectra are observed from Figures 7.21 through 7.24 in most cases, not to follow the minus five-thirds law at the high frequency end of the spectrum within the range of measurement. Though this is not a universal characteristic of the turbulence and could be peculiar to the particular terrain features of the Hanford site, a similar wide variability of the inertial subrange inception might be anticipated at other sites. If turbulent energy is being added within a wave number range from an external source as well as cascaded to hiqher wave numbers by inertial transfer, the negative slope must be less than two-thirds. However, if energy is being removed from the turbulence in a given component, the negative slope of the logarithmic plot within the range of concern must be greater than 
two-thirds. The observed decrease in lateral component spectral density with increasing wave number, greater than expected from inertial transfer, indicates energy is being removed from the $v^{\prime}$ component turbulence in the wave number region where the other components demonstrate no removal or addition. Cospectral data support this contention as described in the following discussion.

A very tentative possibility for explaining the removal of energy from the $v^{\prime}$ component turbulence is the feeding of energy into the mean motion. Just as the $\overline{u^{\prime}} w^{\prime}$ Reynolds' stress withdraws energy from the mean motion and supplies it to the turbulence in the $u^{\prime}$ component, the $\overline{v^{\prime} w^{\prime}}$ Reynolds' stress can work with the vertical gradient of the mean lateral component, transferring energy from the turbulence in the $v^{\prime}$ component to the mean lateral wind component. This results in the maintenance of a wind direction shear. Thus the mechanical energy term, $\overline{v^{\prime} w^{\prime}} \frac{\partial \bar{v}}{\partial z}$, should not necessarily be ignored in the energy budget Equation (6.2). The existence of considerable organized correlation in the $\mathrm{nC}_{\mathrm{vw}}(n)$ cospectral plots up to $\mathrm{nz} / \overline{\mathrm{U}}=3.0$, beyond the point where the other cospectra have fallen to zero, supports the contention that this Reynolds' stress is removing energy from the $v^{\prime}$ component turbulence in the same region of wave numbers. The mean wind direction shears, though the measurements were not adequate for quantitative evaluation, were of the proper sign required for feeding the mean motion. In all 
unstable and neutral cases during the first series of tests conducted on two consecutive days, the wind backed 10 or 15 degrees between 7 and 400 feet. The increase of the lateral mean wind component with height requires a net negative $\overline{\mathrm{v}^{\prime} \mathrm{w}^{\prime}}$ covariance, demonstrated by two calculations where the high pass filter was left out of the Reynolds' stress program allowing the covariance at low wave numbers to be included. The calculations with the filter in the program gave a positive covariance for these two cases as well as for all but one of the other tests performed on these two days because the low frequency covariance was filtered out. The $\overline{\mathrm{V}^{\prime} \mathrm{w}^{\prime}}$ cospectra likewise show the neqative correlation at low wave numbers and a positive correlation at high wave numbers. Such a distribution not only removes energy from the turbulence at the upper end of the spectrum but supplies it at low wave numbers, contributing to the considerable variability detected in the lateral component spectra.

There are exceptions to the behavior discussed above. Tests $11-1$ and $11-2$, in neutral and unstable conditions respectively at 6.1 meters, both follow the minus fivethirds law beyond $n z / \bar{U}=3.0$ and both have essentially no correlation between the $v^{\prime}$ and $w^{\prime}$ components over the range of frequencies filtered for cospectral density. Thus the absence of energy withdrawal from the turbulence to support a mean lateral gradient allows the turbulence to become 
isotropic at an earlier stage.

None of the stable tests show any sign of an inertial subrange even though the cospectral densities are quite small and no organized area under the $\overline{v^{\prime} w^{\prime}}$ cospectral curves near $\mathrm{nz} / \overline{\mathrm{U}}=3.0$ is obvious which might transfer energy out of the turbulence. However, wind direction shears, which were not adequately measured here, are often very large in stable situations and small cospectral areas might yet be effective in altering the low level of turbulence present in the stable cases.

Tests above 12 meters show only a limited agreement with the minus five-thirds law. The light wind unstable case, Test 12-1 agrees quite well above $n z / \bar{U}=1.0$ and the 87 meter case, Test $13-1$ is not inconsistent with the law above $n z / \bar{U}=10$ but the other tests deviate noticeably throughout the range of measurement.

Summarizing the significant factors of the spectra briefly, the normalized spectra for each component have a relatively simple and repeatable dependence on $n z / \bar{U}$. There are, however, differences from one component to another which is to say that a different similarity relationship is observed for each component. This similarity is most marked for the vertical component and poorest for the lateral component. At low wave numbers the apparent similarity might be questioned because of the large variability of the estimates. All, demonstrate a slight shift to higher wave 
numbers over the entire spectrum for stable conditions near the ground and a large shift for the stable case at 12.2 meters. Also convective peaks in the vertical component spectra are found in unstable cases above 12 meters at low wave numbers while the mechanical energy peak and the upper end of the normalized spectrum correspond to the normalized neutral spectrum. A transfer of turbulent energy to the mean flow is indicated in the lateral component spectra for many tests, shifting the lower end of the inertial subrange to higher wave numbers. Otherwise it qenerally begins at about $\mathrm{nz} / \overline{\mathrm{U}}=3.0$ although the minus five-thirds law for the vertical component extends down to $n z / \bar{U}=0.8$ to 1.0 and for the longitudinal component, down to $\mathrm{nz} / \overline{\mathrm{U}}=0.2$ to 1.0 .

The features of the spectra for the tests at 3.0 and 6.1 meters can best be seen from pooled results for each component. These are shown in Figure 7.38 for the longitudinal component, Figure 7.39 for the vertical component, and Figure 7.40 for the lateral component. Average curves for neutral, unstable and stable cases are shown in each figure with the 808 confidence interval indicated with shading for each stability group, reflecting the reduced uncertainty of the data resulting from the averaging. Excluded from the lateral component averages for the neutral and unstable cases are Tests $11-1$ and 11-2, respectively, which were obviously not similar to the others. In addition to demonstrating more clearly (particularly at low wave 
numbers) the features of the spectra previously discussed, such as the insignificant difference between neutral and unstable cases within the forced convection region and a noticeable shift to higher wave numbers for stable cases, it is also clear that the shift of the stable curves necessary to maintain similarity is not the same for all components. For stable cases the normalized $v^{\prime}$ spectrum is similar in shape to that for neutral cases but is shifted to higher $n z / \bar{U}$ by a factor of 2.0 . For the $u^{\prime}$ component the factor is 1.5 and for the $w^{\prime}$ component the factor is 1.1 .

The Universal Constants of the Inertial Subrange

Evaluation of the universal constant, $a$, in the expression for the inertial subrange was made for individual tests in addition to the comparison, previously discussed, between Equation $(6.3 \mathrm{~b})$ and Figures $7.5,7.6,7.13$ and 7.14 using the generally accepted value of $a=0.138$. The evaluations, using Equation $(6.3 \mathrm{~b})$, were made independently for each test from the longitudinal spectra and with the additional factor of $4 / 3$ in the equation, from the vertical spectra for each test conducted at 3.0 and 6.1 meters in neutral conditions. The results are listed in Table 7.1 . The estimates of the universal constant, a, are reasonably close to one another for a given component but the average values from the two components differ considerably, the average value of 0.163 from the longitudinal spectra being 
somewhat high and the value of 0.129 from the vertical spectra being somewhat low. It must be stated that the factor of $4 / 3$ required between $n S_{u}(n) / u \star 2$ and $n S_{w}(n) / u \star 2$ in the inertial subrange for neutral conditions was not found but on the average these quantities were essentially the same for the two components. The same result, not yet published has recently been found by $R$. W. Stewart. This information was received through a personal communication with Prof. H. A. Panofsky. The dependence of the spectral density in the inertial subrange on $\varepsilon^{2 / 3}$ however, is illustrated very well since $u^{*}$ extends over a range of nearly an order of magnitude and $\varepsilon$, therefore, over a range of nearly three orders of magnitude. Indeed, the close organization of the spectra for both the longitudinal and vertical components in the inertial subrange and the constancy of the ratios $\sigma_{u} / u^{\star}$ and $\sigma_{w} / u^{*}$ for neutral conditions, testifies through Equation $(6.3 b)$ to the dependence on $\varepsilon^{2 / 3}$

For the unstable cases within the forced convection region, considering that the dissipation can still be represented by $u^{\star 3} / \mathrm{kz}$ (the wind speed profiles appeared very closely logarithmic), the value of "a" was again calculated from both longitudinal and vertical component spectra and are listed in Table 7.1. The values obtained from vertical spectra, averaging 0.123 , are about the same as those calculated for the neutral cases, slightly lower than expected. The values from the longitudinal spectra, 
averaging 0.131 , are seen to be, in this instance, auite consistent with those obtained from vertical component spectra. The reasonable and consistent results obtained in unstable conditions in the region of forced convection again demonstrates the dominance of mechanical energy production over the convective energy production under these conditions.

Only two cases for the lateral component could be used for evaluating "a" from the spectra beyond $n z / \bar{U}=3.0$. These produce an average value of 0.137 .

In stable conditions even close to the ground, an evaluation of the constant, $a$, through Fquation (6.3b) produces estimates much too large indicating that Equation (6.3b), with its limitations, is inappropriate to the stable case. If all the effective terms were known for the energy equation in the stable cases besides the mechanical energy production term, leading to a realistic expression for the dissipation, an adequate expression for the normalized spectra could be obtained in the inertial subrange, comparable to Equation (6.3b), for cases other than those where mechanical energy dominates. Then "a" could be calculated similarly for stable cases. Future tests with additional instrumentation should provide this capability.

Using the dimensionless wind shear, $\mathscr{B}(\mathrm{R} i)$, calculated from the average Richardson's numbers, and the average ratios of the variances to $u^{\star 2}$, with the average curves for the diabatic cases in Figures 7.38 and 7.39 , Equation (6.3c) 
was tested. The agreement between unstable and neutral cases was slightly improved, yielding an average value for the constant, $a$, of 0.15 and a separate check with the more unstable test $12-2$ from 12.2 meters gave the same value. The stable cases, however, gave values of "a" much too low, indicating the assumptions used in arriving at Equation (6.3c) were invalid for the stable case.

\section{Comparison with Previous Results}

Organizing spectral, estimates as a function of wave number rather than frequency is not a recent notion. Taylor's hypothesis, demonstrating that for a given height spectral distributions can be presented as functions of $n / \bar{U}$ has been verifled in the laboratory for homogeneous conditions by Favre, Gaviglio and Dumas ${ }^{12}$. Panofsky, Cramer and $\mathrm{RaO}^{32}$ have shown that, in the fiald, space correlations and autocorrelations can be matched through Taylor's hypothesis close to the ground for lag distances less than 90 meters.

Further verification of Taylor's hypothesis is qiven by a comparison of afrcraft and tower measurements of turbulence made by Lappe, Davidson and Notess, described by Panofsky and Press35. Measurements at 90 and 102 meters demonstrated the validity of the hypothesis for the longitudinal and vertical components except for discrepancies in the energy at low "frequencles for the vertical component that 
could be explained on the basis of surface inhomoqeneities or slow variations in the mean wind speed. As a result, Taylor's hypothesis would be expected to apply through the range of analysis in the present study which extends down to $0.012 \mathrm{cps}$.

The scaling of the turbulence spectra with height was first noted by Panofsky ${ }^{31}$ for the vertical component. Later Panofsky and McCormick ${ }^{34}$ compared vertical component spectra from various sites, detecting similarities when the spectra were plotted as functions of $\mathrm{nz} / \overline{\mathrm{U}}$ for heiqhts ranging from less than one meter to several hundred meters. Gurvich15 likewise comparedvertical component spectra for heights of one and four meters and found matching curves when plotted as a function of $\mathrm{nz} / \overline{\mathrm{U}}$.

An $n z / \bar{U}$ dependence for the other components has been looked for by a number of investigators with mixed results. Generally, the analyses have indicated agreement with the $\mathrm{nz} / \overline{\mathrm{U}}$ deperidence required in Equation (6.3b) for the inertial subrange but at lower wave numbers some height dependence remains. Davenport ${ }^{9}$ found longitudinal spectra from various heights best organized independent of height. Webb 44 . Henry, and Cramer ${ }^{6}$, (as described by Lumley and Panofsky) all find some height dependence but not a simple linear scaling of the turbulent eddy lengths. Berman ${ }^{2}$ has most effectively organized existing data for the longitudinal component spectra, collected in previous field programs at 
O'Neill, Nebraska, at Brookhaven, Long Island, and at Round Hill, Mass. Also included were a large number of spectra collected by Davenport and by Zubkovsky. Berman plotted normalized spectral estimates, $\mathrm{nS}_{\mathrm{u}}(\mathrm{n}) / \mathrm{u}^{\star 2}$, as a function of $\mathrm{nz} / \overline{\mathrm{U}}$ and demonstrated an additional height dependence most pronounced at low wave numbers and disappearing as the inertial subrange was approached where Equation (6.3b) must hold. The spectral peaks for neutral conditions were found by interpolation to occur at $n z / \bar{U}$ values varying proportional to the 0.75 power of height. Although in the present investigation no additional height dependence was detected, the uncertainty of the estimates is too great to contradict Berman's conclusions.

The lateral component spectra have been found in previous studies to be even less dependent on height than the spectra for the other components. Panofsky and Deland 33 demonstrated that at both Brookhaven and O'Neill, the lateral spectra were strongly dependent on the stability and rather insensitive to height changes. However, this effect occurred at low wave numbers and there is an.indication from a plot of lateral spectra from Brookhmen (Iumley and Panofsky ${ }^{20}$, in stable conditions that at higher wave numbers, a dependence on $n z / \bar{U}$ occurs. The presence of mesoscale eddies in the lateral and longitudinal turbulence and the occurrence of the spectral peaks at low frequencies lexcept for lateral spectra in stable conditions) causes a large 
fraction of the turbulent energy to be quite variable in magnitude at a given low wave number and difficult to sample adequately. Consequently it is not surprising to find differences in the amount of turbulent energy at low wave numbers, resulting both from real mesoscale influences and from wide statistical variability. In this study also, for the lateral component at wave numbers below $n z / \bar{U}=0.1$ a great deal of variability in the spectral estimates for neutral and unstable cases is present, obscuring similarity in this region. The filtering out of the very low frequency energy below 0.001 or 0.002 cps along with dc levels in the signals when the total variances were calculated in the present investigation may have helped to unify the data in each group of horizontal component epectra by removing the highly variable, large amplitude, low frequency trends and meander. The shape of the spectra and the location of the spectral peaks for the vertical component in the present investigation do not differ greatly from prior reports of such features. The comparison by Panofsky and McCormick 34 of data from various sites show peaks in $\mathrm{nS}_{\mathrm{w}}(\mathrm{n})$ ranging from $\mathrm{nz} / \overline{\mathrm{U}}=0.2$ to 0.6 , agreeing quite well with the occurrence of the $w$ spectra peaks, here, at about $n z / \bar{U}=0.4$. Similarly, the present work agrees with the sharp drop-off previously found for turbulent energy in the vertical component at low wave numbers, well before $n z / \bar{U}=0.01$. Normalized vertical 
velocity spectra reported by Gurvichl5 also show spectral peaks in the same $n z / \bar{U}$ region, a distribution for neutral conditions showing the same shape throughout but slightly higher in normalized spectral density than the vertical component spectra reported here. The unstable case given by Gurvich agrees over the upper end of the spectrum but the peak value of $n S_{w}(n) \sigma_{w}{ }^{2}=0.33$ is considerably higher than the value of 0.22 for the mechanical energy peak in the present study. The peak found by Gurvich was also at a somewhat lower wave number and was quite possibly a convective peak occurring near and obscuring the mechanical energy peak. His stable case is definitely shifted to higher wave numbers than for neutral and unstable situations, more pronounced than the slight shift noted in the inertial subrange for the three and $\mathbf{s i x}$ meter stable cases in the present study.

The empirical equation suggested by Panofsky and McCormick,

$$
\frac{n S_{w}(n)}{\sigma_{w}{ }^{2}}=\frac{4 n z / U}{(1+4 n z / \bar{U})^{2}}
$$

has the same shape in the energy producing region as the vertical component spectra for the forced convection region in this report but is shifted to lower wave numbers by about 408 and as Panofsky ${ }^{20}$ points out, it also does not fit the inertial subrange. It is encouraging, however, to find a similar shape suggested from other data in the low wave 
number end of the spectrum. This is partjcularly true since convective energy is added, not as a function of $n z / \bar{U}$ but can appear, generally undistinquished from mechanical turbulence, anywhere at low $n z / \bar{U}$ values and produce varying spectral shapes for measurements taken above the forced convection region. Identification of convective energy peaks has been difficult and statistical reliability has been poor, though the investiqations of Webb 44 and of Panofsky and Van derHoven 35 have suggested separate peaks at low frequencies suspected of resulting from buoyant energy producticn. In the present study, the convective peaks noted for the unstable case at 12.2 meters and that at 87 meters might be questioned for lack of a quantity of similar confirming experimental results even though they are statistically significant on the $80 \%$ level as separate peaks. But the displacement of this spectrum at low wave numbers, well above the universal form of the spectra for tests in the forced convection reqion, testifies to the presence of energy in addition to that introduced into the turbulence through mechanical feeding, leaving little doubt of its convective origin.

For the horizontal component comparison of spectral shapes allow some general observations to he made. First, for the horizontal component spertra, the peaks have been found at considerably lower wave numbers than for the vertical component. St bility has been shown to effect the shape of the liw wave number end ct tle spectrum, somewhat more 
energy being present in unstable conditions than in neutral and stable conditions, but for all stabilities a preponderance of energy is found at low wave numbers. A bias of the longitudinal spectra to low wave numbers, in relation to vertical spectra, was also found at Hanford and the increase in low wave number energy in unstable conditions agreed also. The occurrence of the spectral peak at about $n z / \overline{\mathrm{U}}=0.03$ agrees with Berman's analysis for longitudinal spectra at 6 meters. Also, his value for the normalized spectral peak of 1.7 is not greatly different from the value of 1.3 found in the present study. Furthermore, Berman's analysis indicated the longitudinal spectra were only slightly shifted to lower wave numbers for unstable conditions while a noticeable shift to higher $n z / \bar{U}$ occurred in stable conditions, a result in agreement with the present study. For the lateral spectra, however, a comparison with Brookhaven and $O$ 'Neill data reveals a marked difference for the stable cases. Though the large amount of energy at low wave numbers for unstable conditions is a comparable feature, the low wave number peaks for the stable cases at Hanford do not compare with some other observations where peaks in stable lateral component spectra occur at about the same wave number as for the vertical component.

At the high wave number end of the spectra, considerable evidence has been accumulated, supporting the minu five-thirds relation of Kolmogoroff. Early measuremente by 
McCready ${ }^{21}$ using hot wire techniques at several heights close to the ground are consistent with kolmogoroff's theory, both in the $r^{2 / 3}$ shape over a reqion of the autocorrelation function and through spectral densities taken at three frequencies. McCready's ${ }^{23}$ recent work with a sailplane likewise supports the minus five-thirds spectral distribution. Businqer and Soumi ${ }^{4}$ in their pioneering work with the sonic anemometer also show agreement with the minus five-thirds law over extended reqions of vertical component spectra. Recent work with the sonic anemometer reported by Gurvich ${ }^{15}$ and Monin 27 also support the existence of an inertial subrange in vertical component spectra. For the horizontal component the recent measurement of Pond, et al. ${ }^{38}$ with as hot wire anemometer present very convincing evidence for the presence of an inertial subrange at one height over water. zubkovsky's measurements of longitudinal component spertra at 4 meters confirm the minus five-thirds law, extending to wave lengths five times the height, as was similarly found in the present study for unstable cases.

The low wave number limit of the inertial subrange has been investigated primarily by determining the point at which the minus five-third law no longer fits observed spectral distributions. McCready ${ }^{22}$ presents summarized results of a number of investigations to show that this point, for the vertical and lateral component spectra, applies to wave lengths on the order of twice the height in the region below 
about 200 meters; $\mathrm{nz} / \overline{\mathrm{U}}$ for this point varies from about 0.8 to 0.4 for stable to unstable condition, respectively. His summary shows some dependence on height; the $n z / \bar{U}$ value for the lower limit of the range increases with height. For the longitudinal component, McCready's summary indicates no stability dependence for the lower $n z / \bar{U}$ limit which occurs about 0.4. Gurvichl5 also gives estimates of the low wave number limit for the minus five-thirds law from his one meter and four meter vertical component spectra. He found for his most stable case ( $R i=0.28$ ) that the limit occurred at $n z / \bar{U}=5.2$, for neutral conditions, at $n z / \bar{U}=0.72$, and for his most unstable case $(\mathrm{Ri}=-0.76) \mathrm{nz} / \overline{\mathrm{U}}=.40$.

The results reviewed above agree in some respects with those of the present study. The lower limits of the minus five-thirds law is generally found to occur at lower $\mathrm{nz} / \overline{\mathrm{U}}$ for longitudinal spectra than for vertical spectra at Hanford as well as elsewhere and some stability dependence is also common. However, the lower limit of the minus five-thirds law is observed in the vertical component spectra at generally higher $n z / \bar{U}$ for Hanford than elsewhere. Furthermore, there is only a very slight dependence of the occurrence of this point on stability at 3.0 and 6.1 meters, while there is a marked shift of the stable case at 12.2 meters to higher $n \pi / \bar{U}$ at Hanford. This suggests that more extremely stable and unstable situations at the 3.0 and 6.1 meter levels might show greater stability dependence. Another 
important factor is that the surface roughness at Hanford, characterized by sage brush averaging one meter in height, is generally greater than at many other turbulence test sites and consequently a region dominated by mechanical turbulence in reasonably strong winds may be deeper than at other sites. Thus differences due to stability at other sites at a height of four meters might not be expected at Hanford even at six meters.

The shift of the lower limit of the minus five-thirds longitudinal spectra with stability $(n z / \bar{U}=1.0$ for stable to $n z / \bar{U}=0.2$ for unstable) is known with about 808 confidence. Results summarized by McCready show no such shift but it is believed that the results reported here are reliable enough to detect the small difference in slope of the curves in Figure 7.38 which lead to the conclusion that there is a shift of the lower limit.

The lack of height dependence of the minimum $n z / \bar{U}$ in the inertial subrange for 3.0 and 6.1 meters at Hanford also differs from results reported by McCready.

The observation in the present study that the minus five-thirds law usually extends to lower wave numbers than the true isotropic limit (where cospectra become zero) agrees with the findings of pond ${ }^{2}$ : On theoretical grounds, Gifford ${ }^{13}$ has also shown thet the one-dimensional spectrum is expected to follow the minus five-thircis law below the true isotropic limit. Confirming measurements of the $\overline{u^{\prime} v^{\prime}}$ 
and $\overline{v^{\prime} w^{\prime}}$ cospectra are lacking in previous studies and the $\overline{u^{\prime} w^{\prime}}$ cospectral measurements are few. However, Monin 27 recently reported results demonstrating the $n z / \bar{U}$ dependence, as well as stability dependence of the $\overline{u^{\prime} w^{\prime}}$ cospectrum which indicated the isotropic limit occurred between $\mathrm{nz} / \overline{\mathrm{U}}$ values of 1 and 10 .

For the longitudinal component from O'Neill data, Elyll demonstrated the dependence of the spectral estimates at high wave numbers on the $8 / 3$ power of wind speed. This dependence is expected from Equation (6.3b) with the logarithmic profile relationship to $u^{*}$, if the spectral estimates obey the minus five-thirds law as well as follow the $\mathrm{nz} / \overline{\mathrm{U}}$ dependence. Thus from

$$
\begin{aligned}
& \frac{n S(n)}{u^{\star 2}}=a k^{2 / 3}(n z / \bar{U})-2 / 3 \\
& \text { with } \quad u^{*}=\bar{U} k / \ln \left(z / z_{0}\right) \\
& \text { we have } n S(n)=a k^{8 / 3}(n a)^{-2 / 3}(\bar{U}){ }^{8 / 3}(\ln z / 2)^{-2}
\end{aligned}
$$

giving the observed dependence on wind speed.

\section{Reynolds' Stress Results}

The Reynolds' stresses, calculated from the wind fluctuation data with the analog computer program discussed previously, are given in Table 7.2. With but a few exceptions, the data are not significantly different from what might be expected. The standard deviation for the fluctuations of 
each of the wind components is divided by the friction velocity as is the mean wind speed at 3.0 meters measured independently with the 80 foot tower equipment, and the ratios are listed in Table 7.2. Though the Reynolds' stresses cover a considerable range of magnitudes, the ratios demonstrate they are consistent and reasonable. The ratio of the 3.0 meter wind speed to friction velocity for neutral conditions varying no more than $\$ 58$ from the average ratio except for one case, demonstrates agreement with the characteristic of the logarithmic profile where the wind speed is proportional to the friction velocity. The average ratio also leads to a roughness length of $z_{0}=2.0 \mathrm{~cm}$., fairly consistent with a roughness length, indicated by Hanford wind profiles, closer to $3.0 \mathrm{~cm}$. Test $6-1$ may have underestimated the friction velocity somewhat because of the short length of record ( $13 \mathrm{~min}$.$) so that the low frequency contributions$ to the $\overline{u^{\prime} w^{\prime}}$ correlation were poorly sampled.

The wind speed at a small height was chosen so that for non-neutral conditions the profile would still be close to logarithmic. The unstable cases including test 12-2 at 12.2 meters give essentially the same ratio as the neutral cases while the stable cases generally give values somewhat larger, indicating the profile is deviating in the expected direction from logarithmic at 3.0 meters for the stable cases. For test $12-1$ all ratios 1 isted are large, as might be expected for such a light wind speed where the momentum 
flux has undoubtedly dropped off somewhat at 12.2 meters. The ratio, $\sigma_{w} / u^{*}$, is also very constant regardless of height or wind speed for the neutral and the 3.0 and 6.1 meter unstable cases with an average value of 1.33 . This value is somewhat higher than the value of 1.25 determined by Panofsky and McCormick ${ }^{34}$ and considerably higher than the value of 1.05 found in wind tunnel investigations cited by Panofsky and Lumley. However, the value here is identical to that found by Pasquill 37 . The increased value of $\sigma_{w} / u$ * in stable conditions noted here has not been observed in other investigations. The unstable test $12-2$ at 12.2 meters gives results not significantly different from the neutral cases.

The ratios $\sigma_{u} / u^{*}$ and $\sigma_{v} / u^{*}$ are more variable than $\sigma_{w} / u^{\star}$ but no dependence on height, wind speed or stability is noted. The lack of dependence of the ratios $\sigma_{u} / u^{\star}$ and $\sigma_{V} / u^{*}$ on wind speed is in agreement with previous observations but investigators have found elsewhere a strong dependence of $\sigma_{\mathrm{v}} / \mathrm{u}^{*}$ on stability as well as a noticeable stability dependence for $\sigma_{\mathrm{u}} / \mathrm{u}^{\star}$.

Some of the differences noted between the variance calculations of this investigation and other reported results can be attributed to the high-pass filtering performed in the analog computer program described earlier. The filter removed fluctuations with periods greater than about 8 minutes for the tests below 12 meters and removed periods 
greater than about 17 minutes for tests above 12 meters. Thus, contributions from low frequency horizontal mesoscale eddies were removed. If the data had not been filtered in this manner, varying amounts of low frequency energy would have appeared in the horizontal component variances depending on the length of record. A number of filters with varying cut-off points were tried. A filter was chosen which would pass the entire vertical velocity variance and the momentum flux without limitation. Consequently, it was considered that all microscale contributions to the turbulence were included. Another difference is the larqe contribution at low frequencies in the lateral wind component variance found at Hanford in stable conditions whereas investigations at other sites have shown a marked lack of low-frequency energy for stable situations.

The $\overline{u^{\prime} w^{\prime}}$ covariance is large, as expected, and is systematically related to the wind speed as described above. Furthermore, the cospectral distributions for the 3.0 and 6.1 meter tests are well organized as a function of $\mathrm{nz} / \overline{\mathrm{U}}$, demonstrating similarity as found in the power spectral distributions. The integrals monitored throughout the analysis (see Fiqures 4.7 and 4.11 ) increase at a steady rate through the tests for both the cospectral estimates and the total covariance calculations, demonstrating little statistical variability.

The $\overline{v^{\prime} w^{\prime}}$ covariances are small, generally less than 
$10 \%$ of the corresponding $\overline{u^{\prime} w^{\prime}}$ covariances, except above 12 meters where the momentum flux has decreased or, for the 37 meter case, where $\overline{u^{\prime} w^{\prime}}$ is reversed in sign, corresponding to a decrease in wind speed with height. Here the $\overline{v^{\prime} w^{\prime}}$ stress transfers the momentum toward the surface corresponding to a wind direction shear at 400 feet. The $\overline{v^{\prime} w^{\prime}}$ cospectral densities are larger than might be expected from the covariance values since in some cases positive contributions at high wave numbers are to a large extent balanced by negative contributions at low wave numbers. Even larger negative contributions are expected for the first series of tests at wave numbers below those passed by the high-pass filter as evidenced by test $6-1$ and $7-2$ where the $\overline{v^{\prime} w^{\prime}}$ covariances were also calculated without the filter. The resulting negative covariances, -0.0864 and -0.0581 respectively were opposite in sign to the filtered values, corresponding to the required wind direction decrease with height noted between 7 and 400 feet during the testing. The integrals monitored in the analysis of both $\overline{v^{\prime} W^{\prime}}$ covariance and cospectral density showed more statistical variability than with the $\overline{u^{\prime} w^{\prime}}$ analysis. However, the integrals generally tended toward the final values. Figure 7.41 shows the increased variability over the $\overline{u^{\prime} w^{\prime}}$ calculation shown previously in Figure 4.11 for the same test.

The $\overline{u^{\prime} v^{\prime}}$ covariances are quite large, but as the cospectral distributions show, the contributions are made 
in a rather disorganized way, occurring both positively and negatively at larger and larger magnitudes for lower and lower wave numbers. In addition, the $\overline{u^{\prime} v^{\prime}}$ covariance and cospectral integrals monitored during the analysis show a distinct lack of statistical regularity, a given integral often reaching both large positive and large negative values at different points in the integration period. The variability is even greater than see in Figure 7.41. Monitoring the analog computer analysis in this way provided insights into the dependability of the estimates obtained. The confidence in the stability of the $\overline{u^{\prime} w^{\prime}}$ covariance and cospectral results as opposed to the hesitance to attach any significance to the $\overline{u^{\prime} v^{\prime}}$ results, derived from monitoring the analog analysis, could not have been supplied by any ordinary test of statistical significance. 
TABLE 7.1

EVALUATION OF UNIVERSAL CONSTANT "a"

(Using $\varepsilon=u^{\star 3} / k z$ only)

From Longitudinal Component

Neutral Cases

Test

5-1

$5-2$

$5-3$

$6-1$

6-2

6-3

$11 \div 1$

Avg Neutral

$\frac{a}{\text { Cycles }}$

0.158

0.147

0.166

0.184

0.172

0.125

0.190

0.538

0.500

0.566

0.626

0.586

0.425

0.646

$0.163 \quad \frac{0.646}{0.555}$
Unstable Cases

Test $\frac{a}{\underline{\text { Cycles }} \text { radians }}$

7-1

0.121

0.412

7-2

0.118

0.401

11-2

0.154

0.524

Avg Unstable $\overline{0.131} \overline{0.449}$

From Vertical Component

Neutral Cases

\begin{tabular}{r} 
Test \\
\hline $5-1$ \\
$5-2$ \\
$5-3$ \\
$6-1$ \\
$6-2$ \\
$6-3$ \\
$11-1$
\end{tabular}

$\frac{a}{\text { Cycles }}$

0.127

0.117

0.120

0.116

0.124

0.152

0.146

0.129
0.432

0.398

0.409

0.395

0.422

0.518

0.497

0.439
Unstable Cases

Test $\frac{a}{\text { Cycles radians }}$

7-1

7-2

0.121

0.126

0.412

11-2

0.122

0.429

0.415

Avg Unstable $\overline{0.123} \overline{0.419}$

Avg Neutral

\section{From Lateral Component}

Neutral Cases

\begin{tabular}{lll}
\hline Test & $\frac{a}{\text { Cycles }}$ & $\frac{\text { radians }}{11-1}$ \\
0.148 & 0.505
\end{tabular}

Unstable Cases

\begin{tabular}{lll}
\hline Test & $\frac{a}{\text { Cycles }}$ & radians \\
$11-2$ & 0.127 & 0.432
\end{tabular}




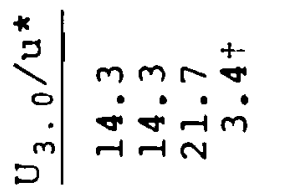

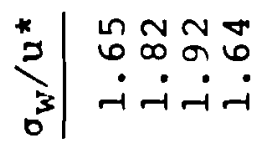

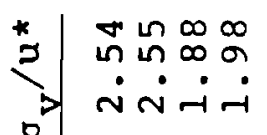

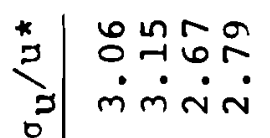

\begin{tabular}{l|lll}
$m$ & -1 & $\infty$ & 0 \\
$r$ & -1 & $n$ & 0 \\
0 & -1 & 0 & 1 \\
0 & 0 & 0 & 0 \\
0 & 0 & 0 & 0 \\
0 & 0 & 0 & 0
\end{tabular}

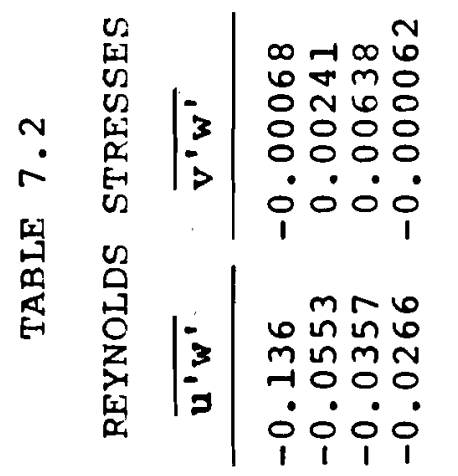

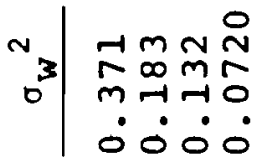

$\sim \mid \begin{array}{cccc}a & 0 & 0 & \sigma \\ N & 0 & v & 0 \\ 0 & m & -1 & -1 \\ 0 & 0 & 0 & 0\end{array}$

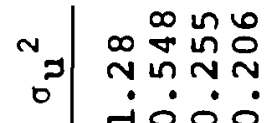

H०

NNmm N

નનનનનનન્ન

mo円

mm

નનનનનન્ન

in $\infty \underset{0}{\infty} \underset{\sim}{\infty}$

! ! ! ?

Nન

mow 6 n

GNR

ن $\dot{\sim} \dot{\sim} \dot{\sim} \dot{\sim}$

요요

$m \infty 6 \pi+N$

OOOROOO

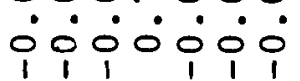

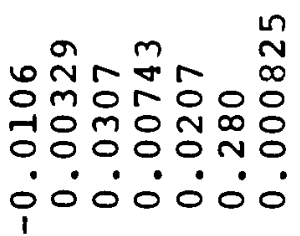

-

or 0 in $\operatorname{co}$

HNMTNO

- 0 0 000

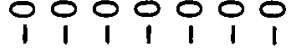

onom

* 6 NNN $N \mathrm{~m}$

$m \forall$ in $\forall$ in

○் ச்

ㅇำ

N

ก!

تं

$m \sim \nleftarrow N \sim \frac{0}{0}$

$00 \mathrm{~m}$ ONO

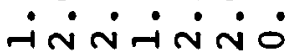

the

$N \sim N$

$न ન-1$

m $m m$

- ?

$ન-1$

060

N $\infty$

Nin

n $-1 m$

กา

N

은

$\mathrm{Nm}$

000

0ं

$\begin{array}{lll}0 & \infty & 0 \\ 1 & -1 & 0 \\ m & 0 & 0 \\ 0 & 0 & 0 \\ 0 & 0 & 0\end{array}$

$m 0 \stackrel{n}{m}$

$m$ in

$\dot{0} \cdot \dot{0}$

000

-10m

कब बन

$0 \infty \pi$

○ं0

$0 \pi-1$

$\infty \infty m$

-i

on

im
$0_{0}^{+}+$

- $0+$

on $\cos t$

다

-

नNA

m듬

- $\cdot$.

$-\nabla N$

กู

i.

vण

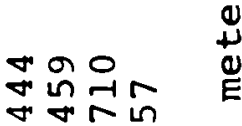

$000 \mathrm{H}$

i 000

N

a $m \infty$

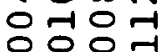

$\therefore: 00$

13

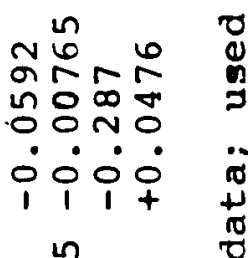

每 n n

ก

다 $\infty$

க்

$\infty N$

nNw

파자

0ं0்-1

융

mं் $\dot{0}$

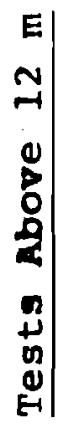

N⿴囗十

$\frac{1}{6}$

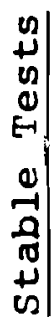

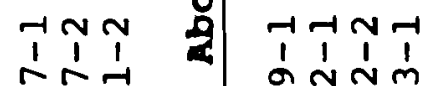

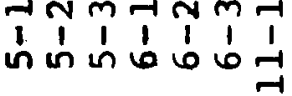




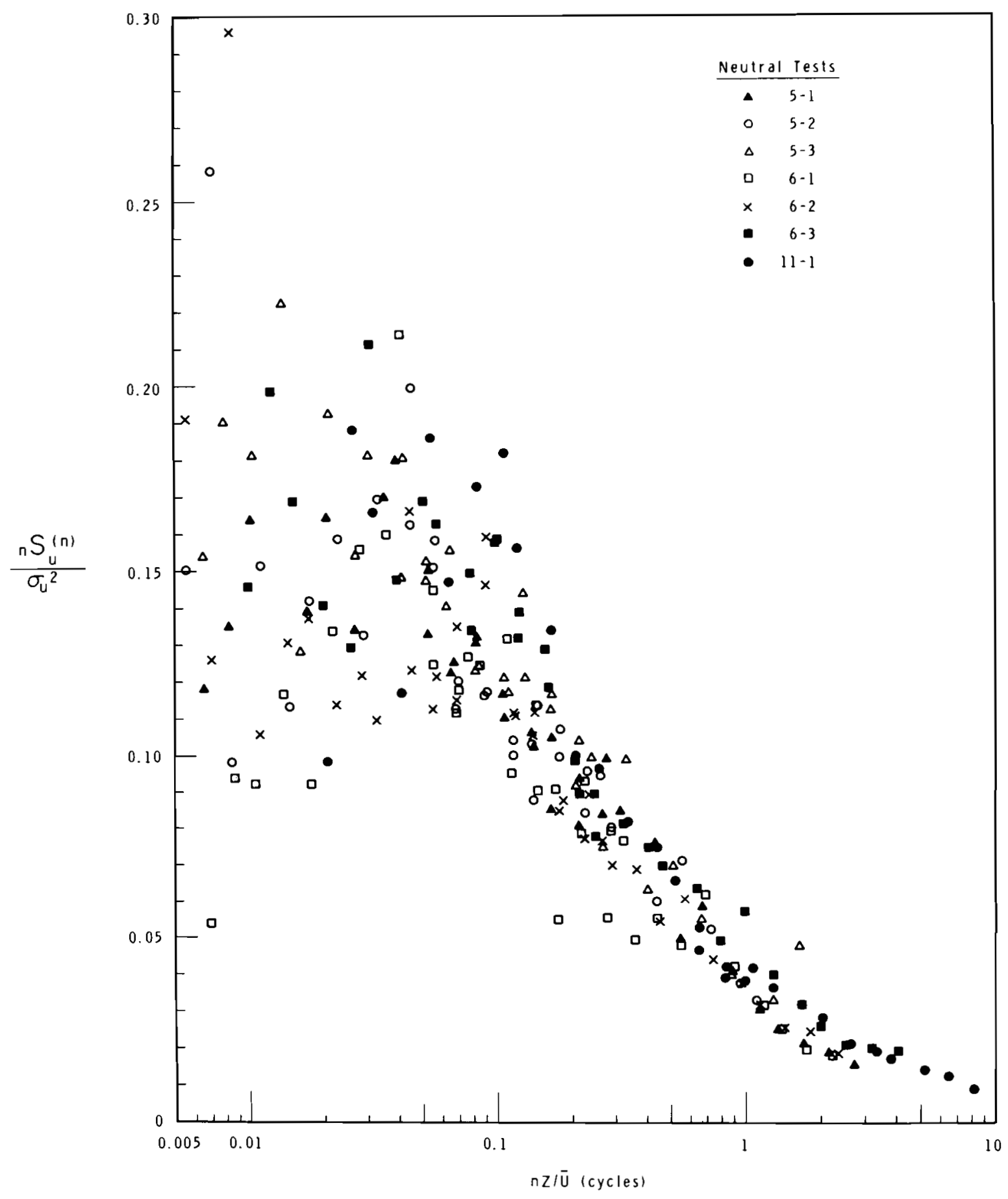

FIGURE 7.1 Longitudinal Wind Component Spectra - Neutral Tests 


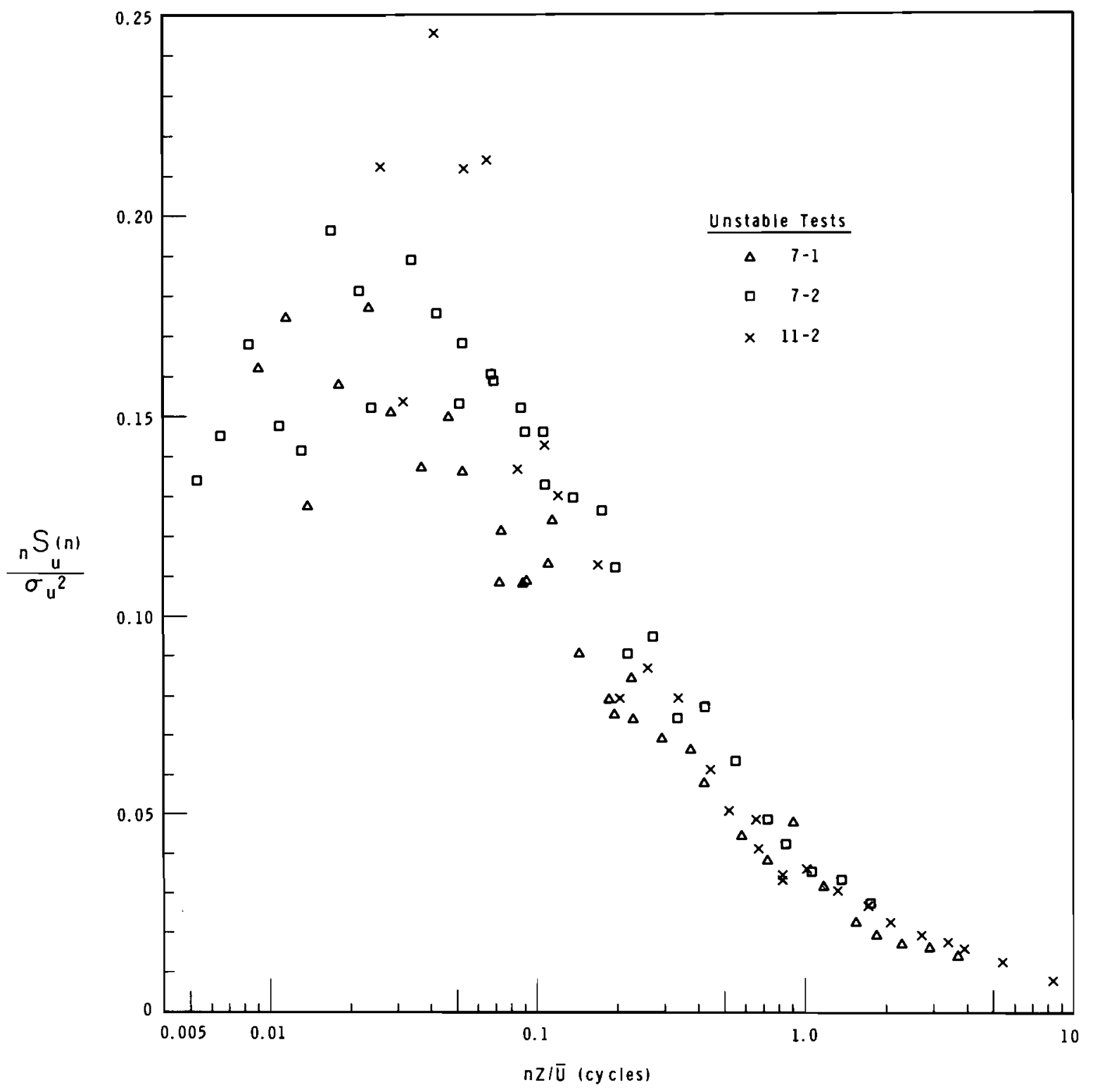

FIGURE 7.2 Longitudinal Wind Component Spectra - Unstable Tests 


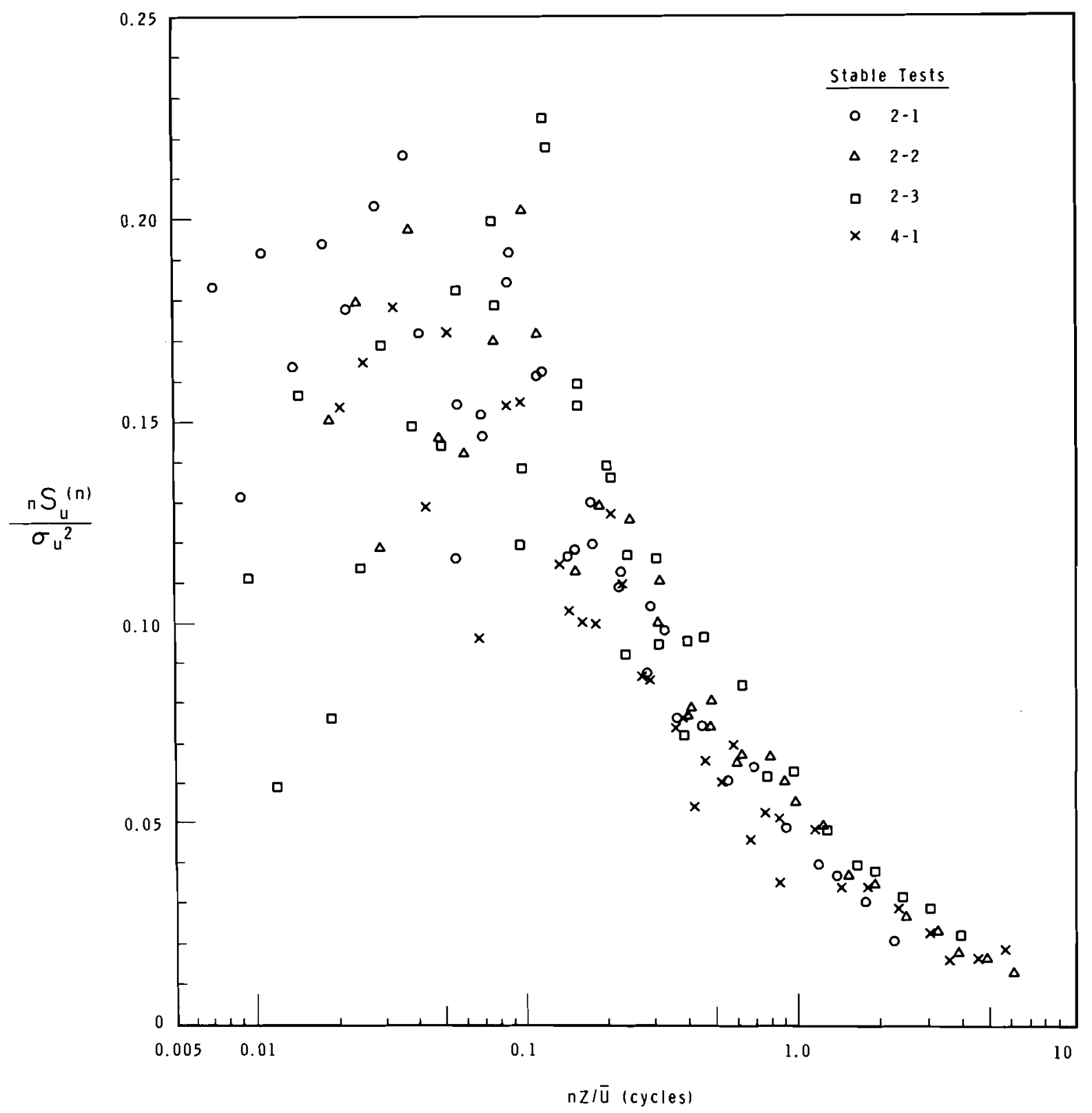

FIGURE 7.3 Longitudina1 Wind Component Spectra - Stable Tests 


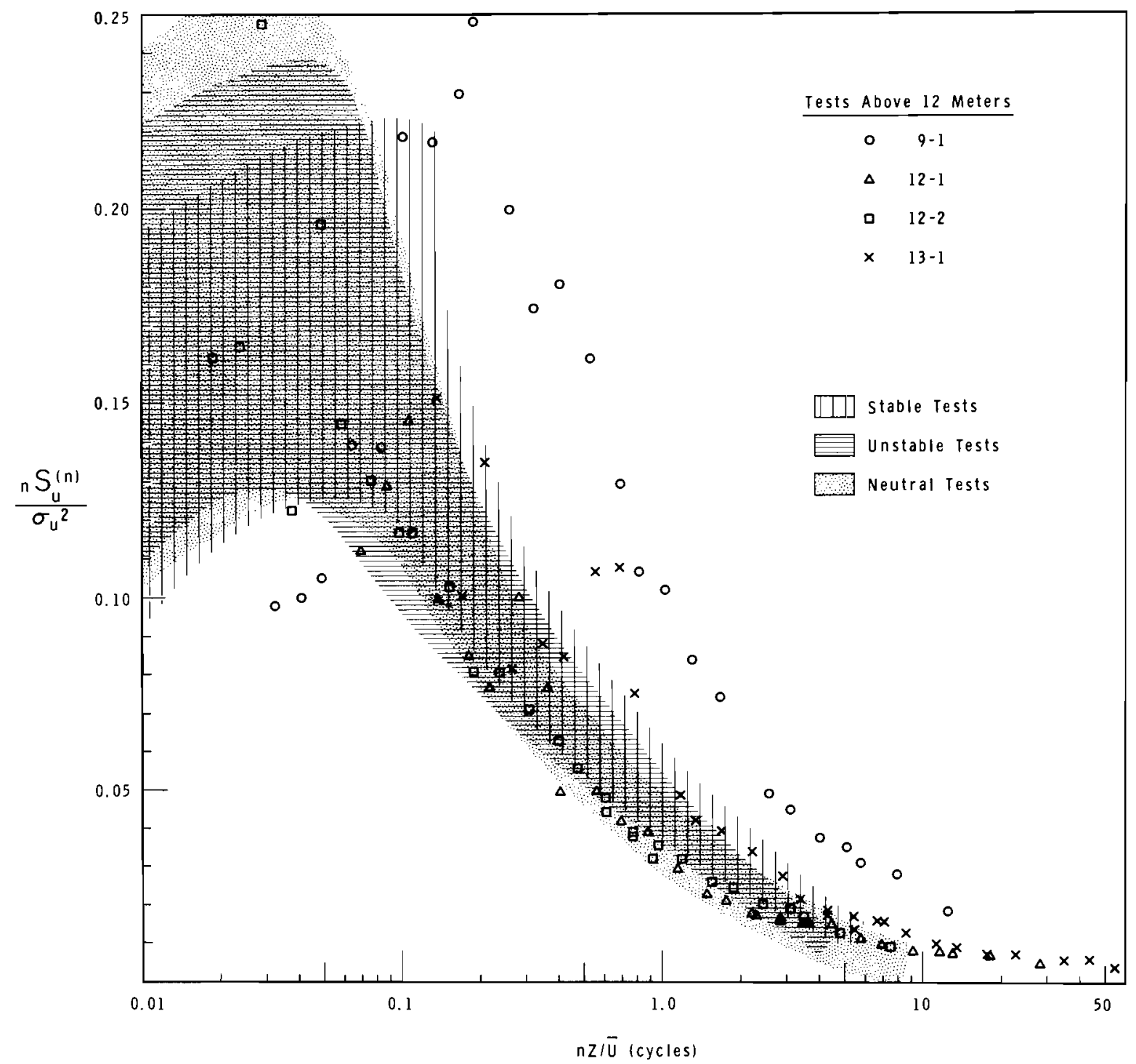

FIGURE 7.4 Longitudinal Wind Component Spectra - Tests Above 12 Meters 


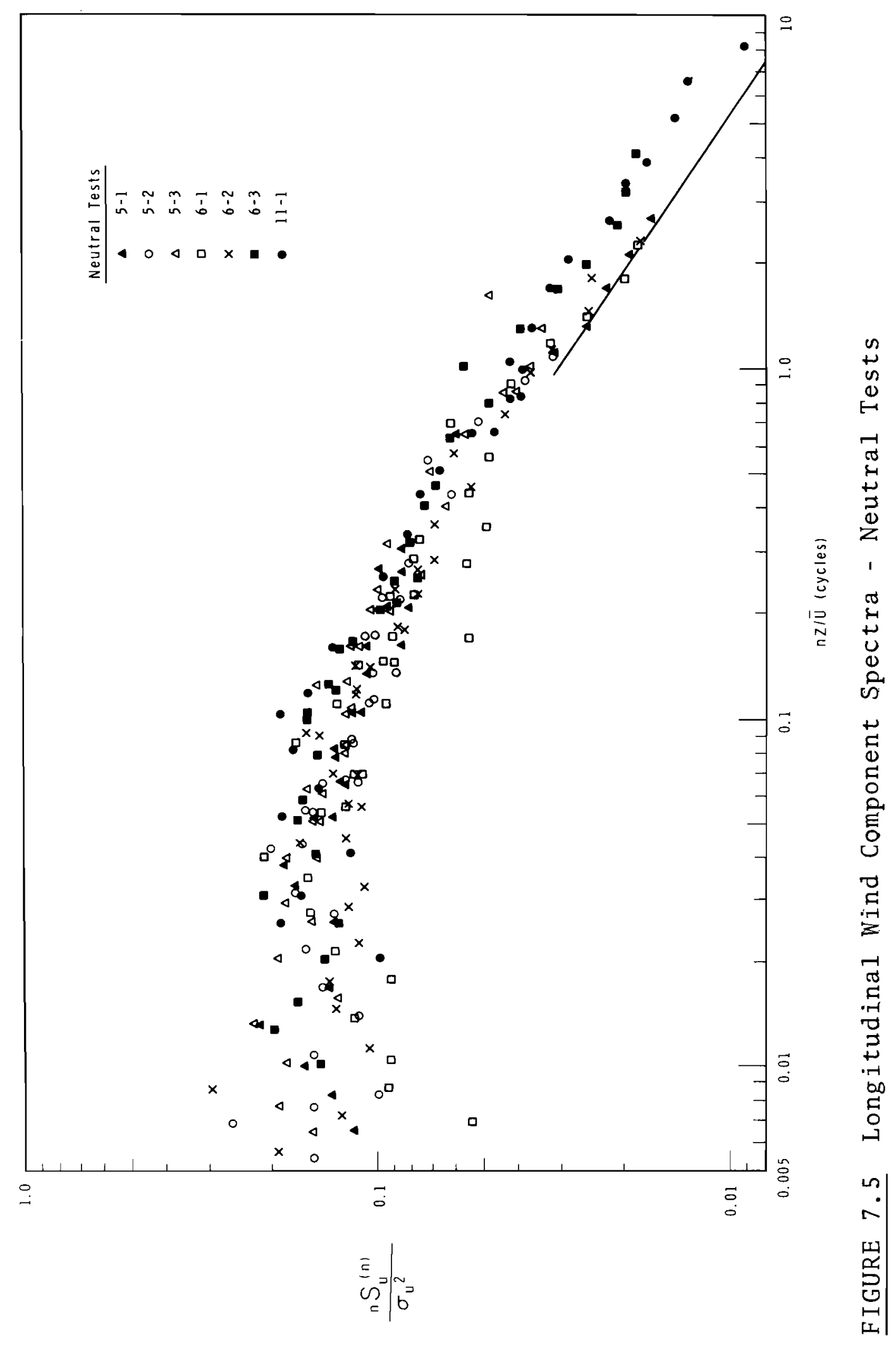




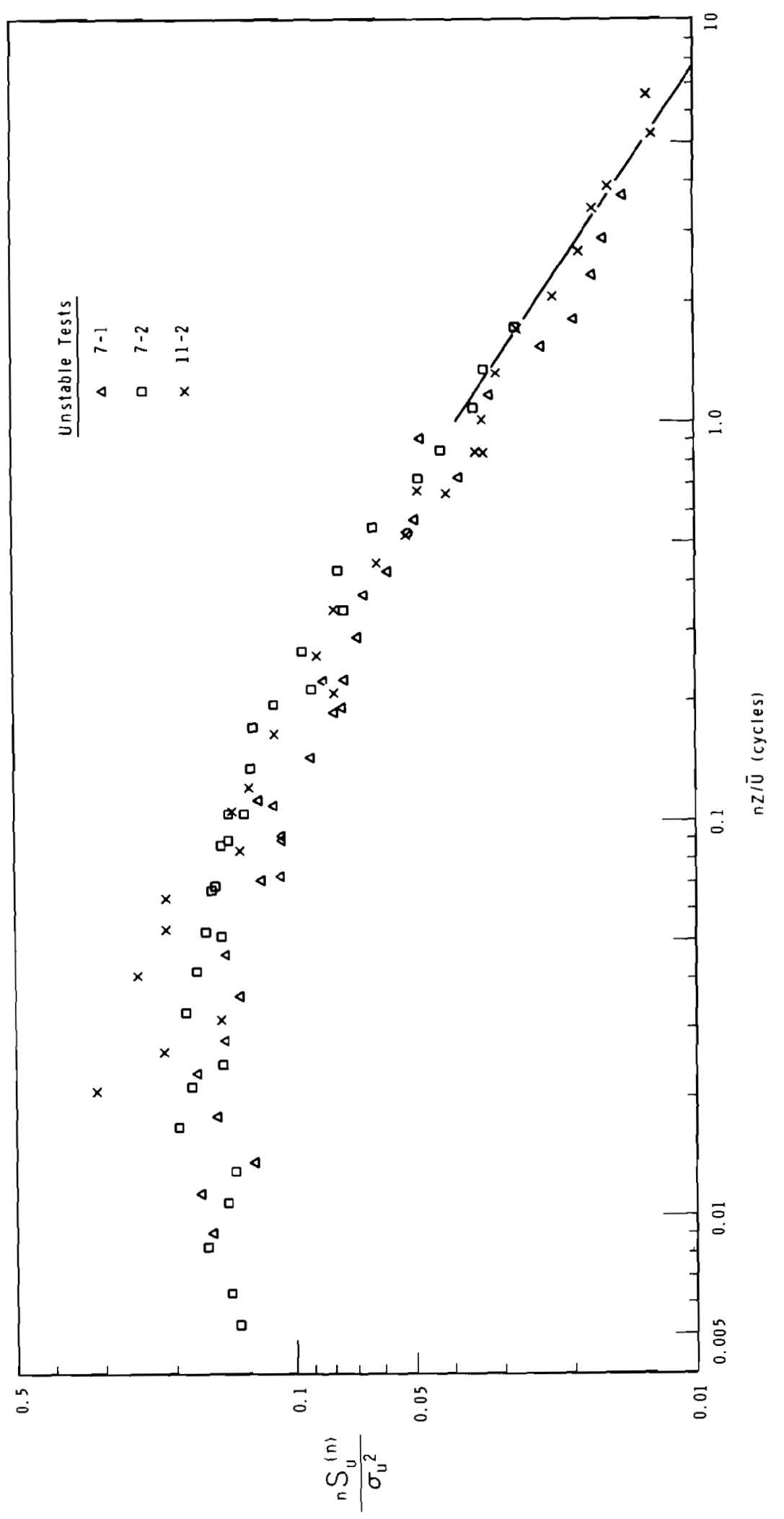

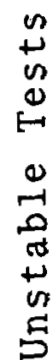

1

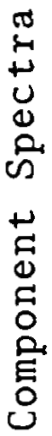

吕

离

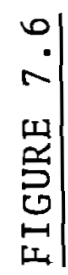




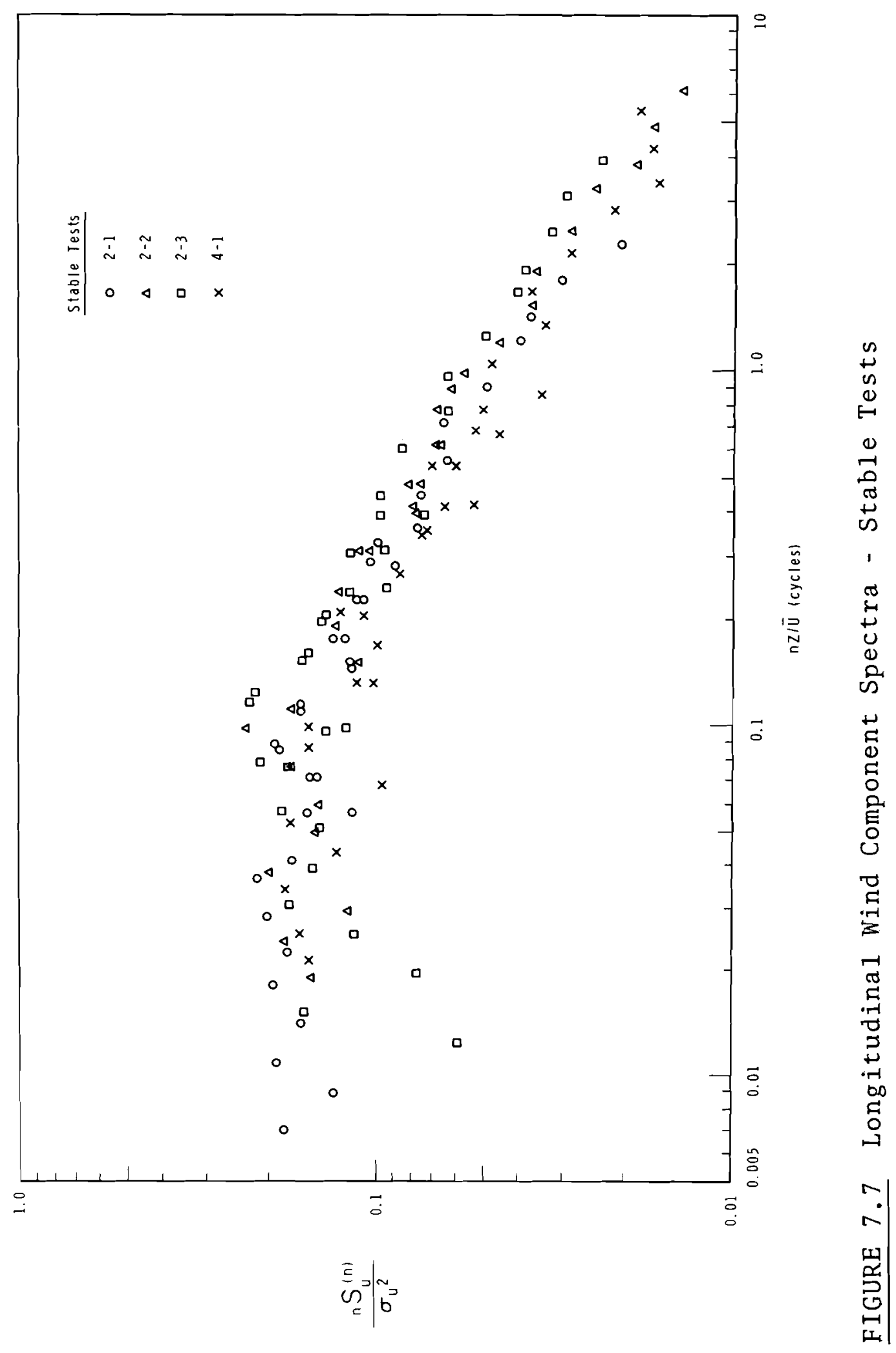




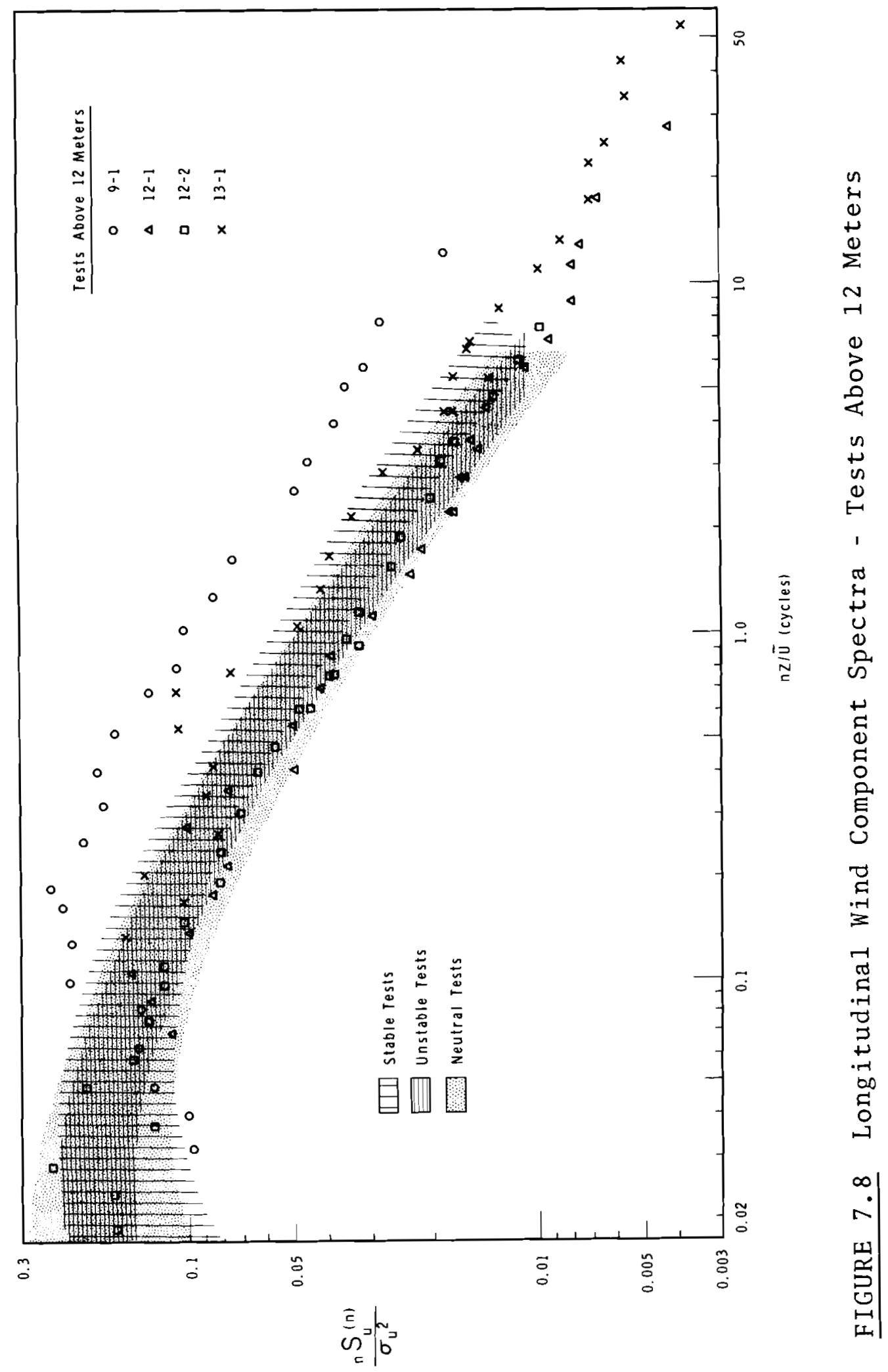




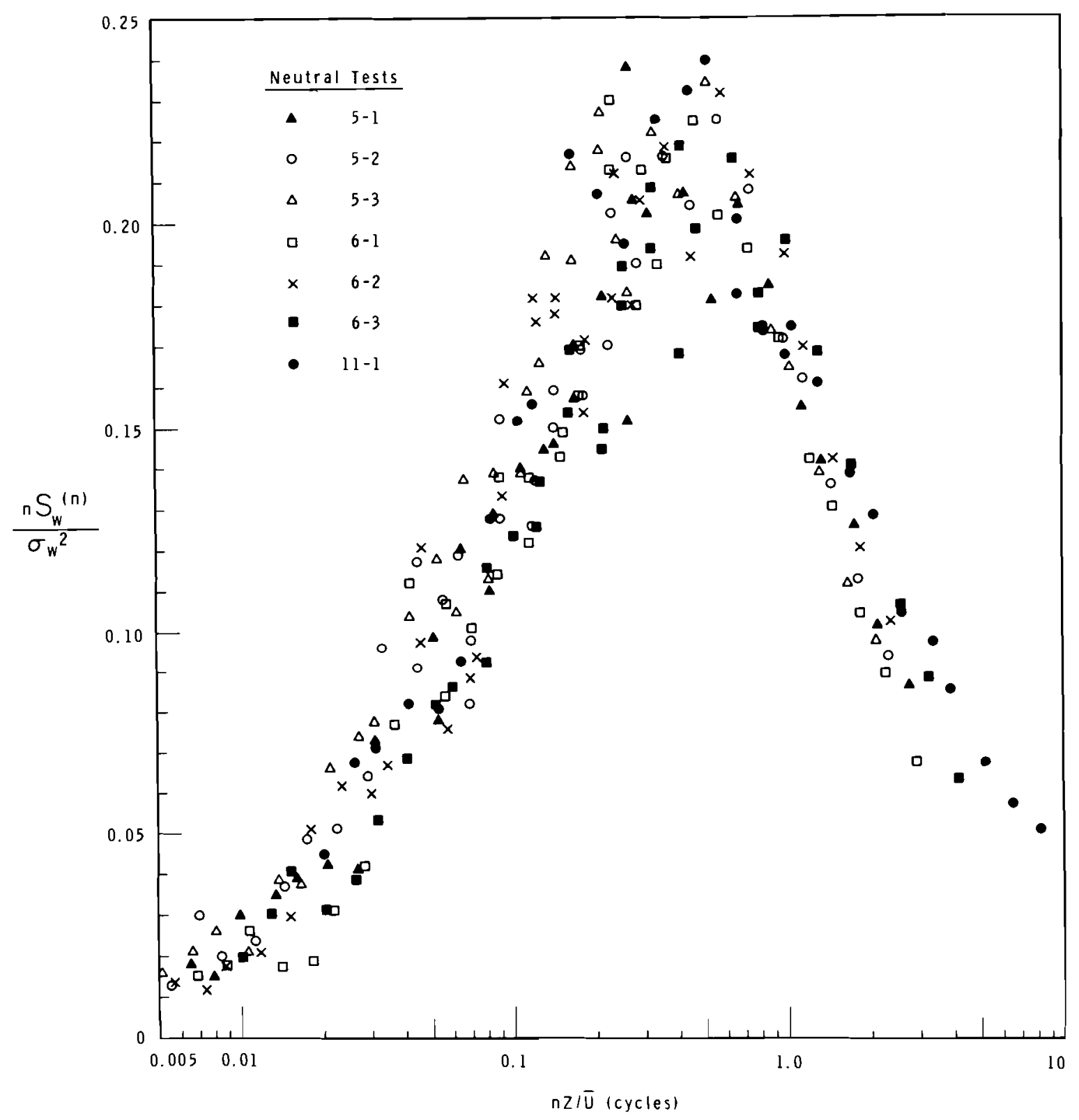

$\underline{\text { FIGURE } 7.9}$ Vertical Wind Component Spectra - Neutra1 Tests 


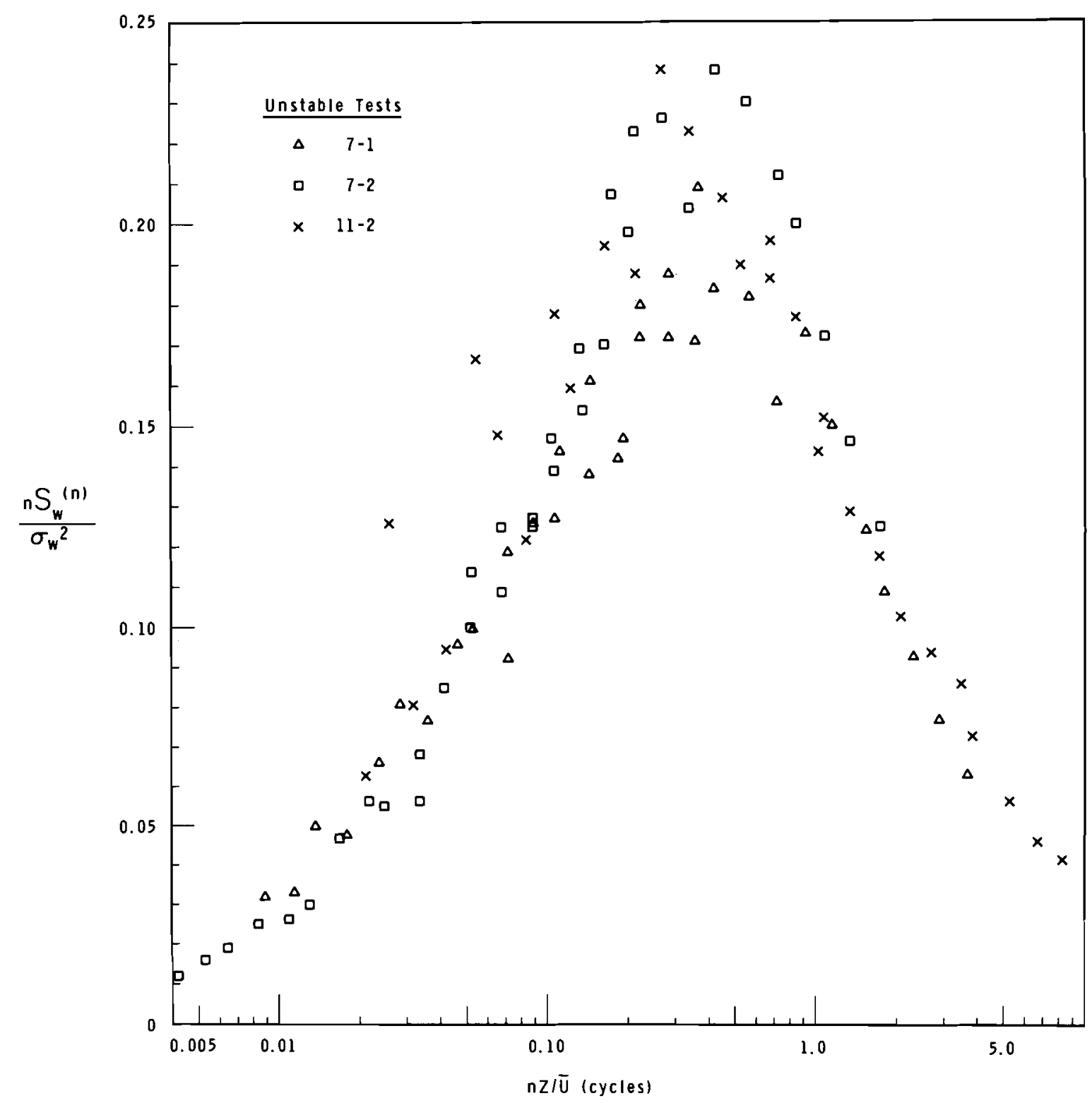

FIGURE 7.10 Vertical Wind Component Spectra - Unstable Tests 


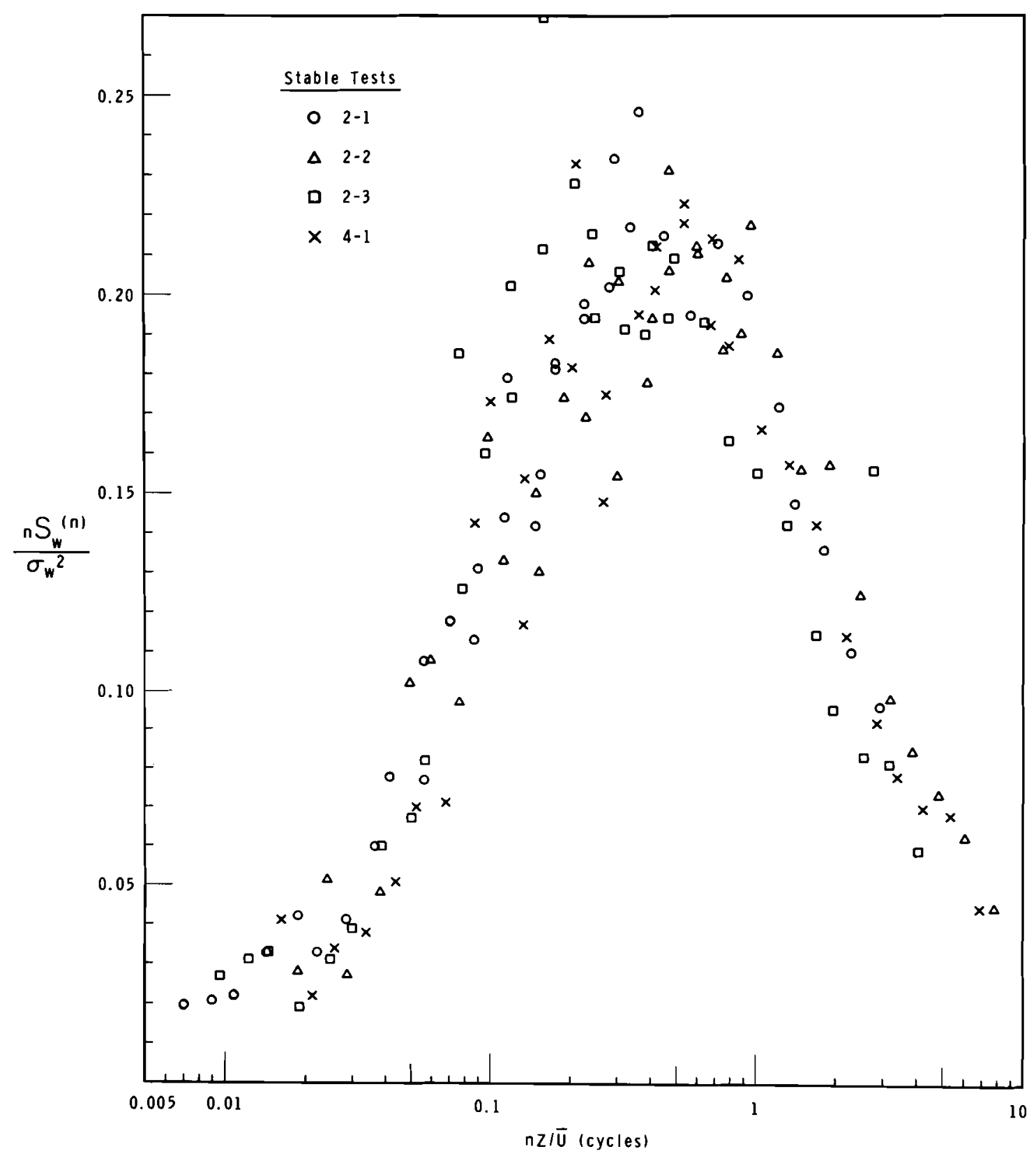

FIGURE 7.11 Vertical Wind Component Spectra - Stable Tests 


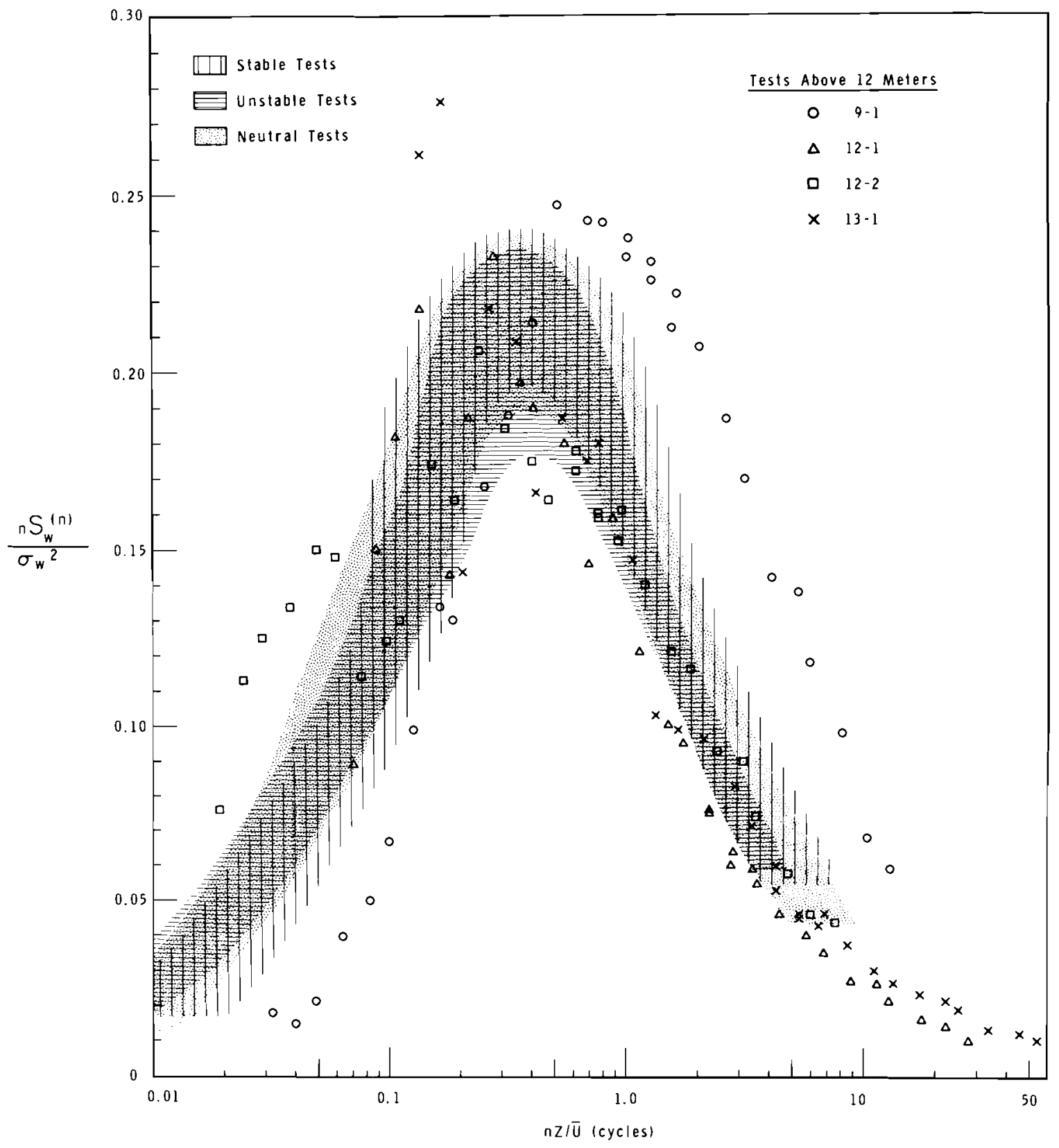

FIGURE 7.12 Vertical Wind Component Spectra - Tests Above 12 Meters 


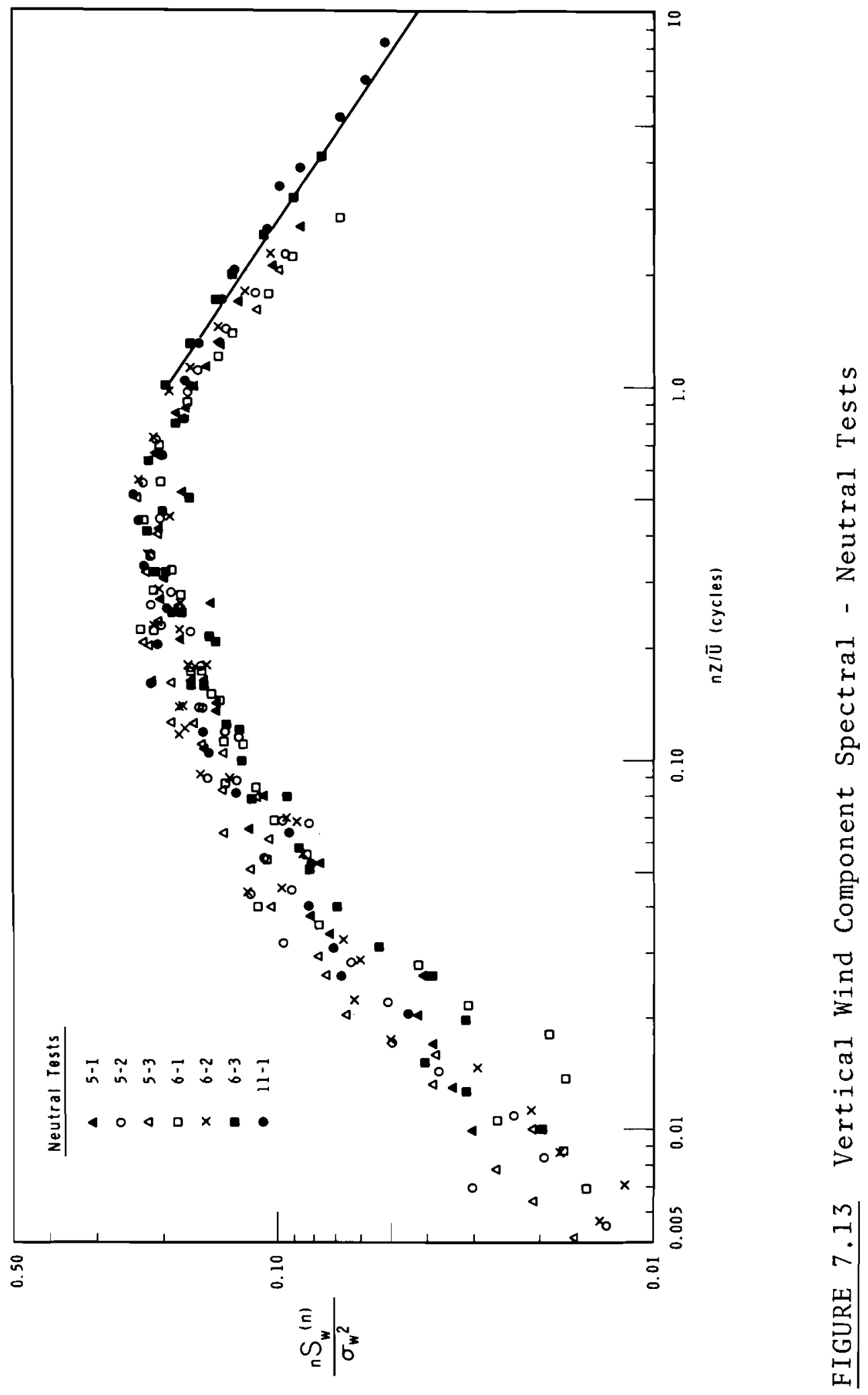




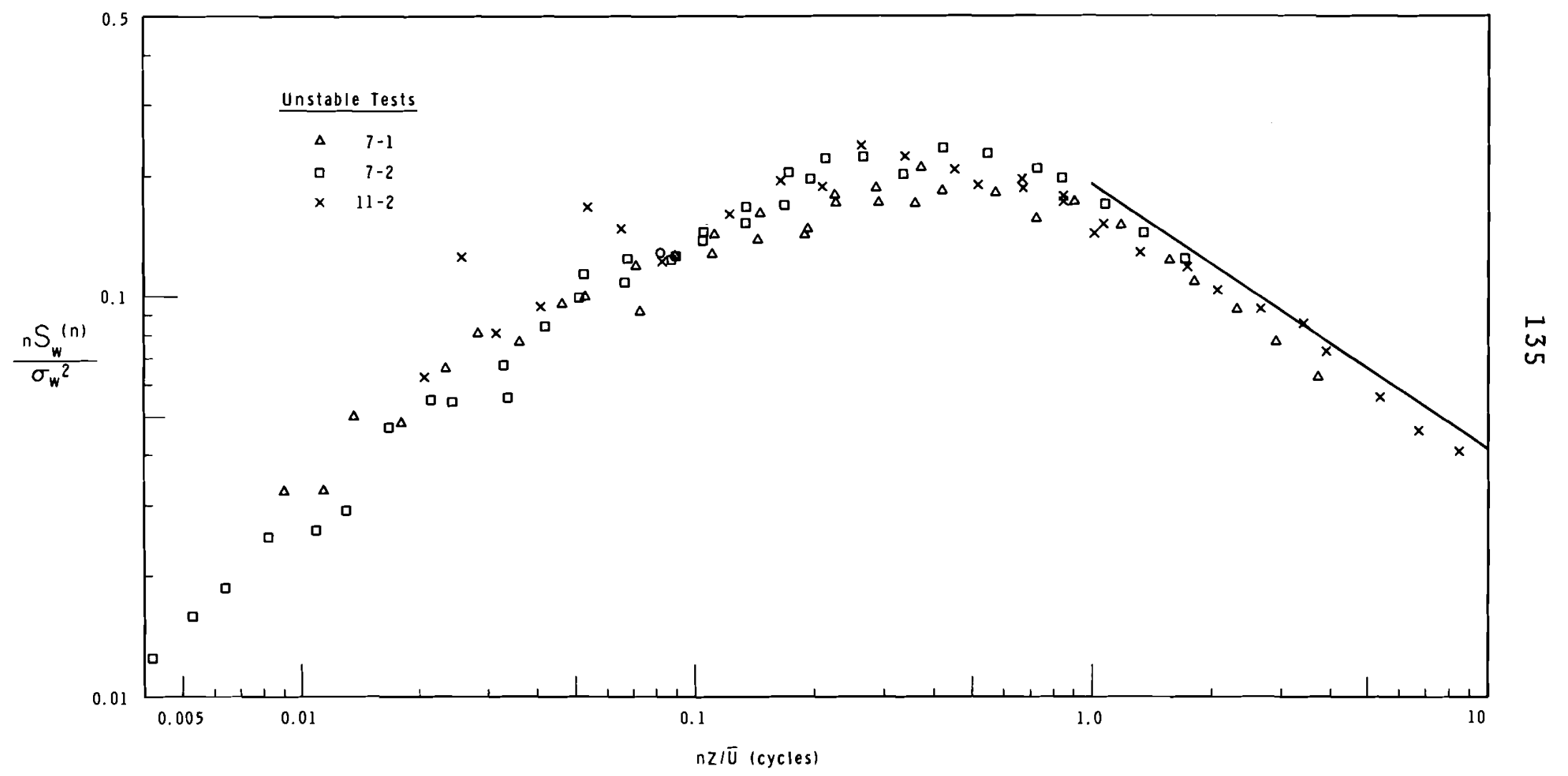

FIGURE 7.14 Vertical Wind Component Spectral - Unstable Tests 


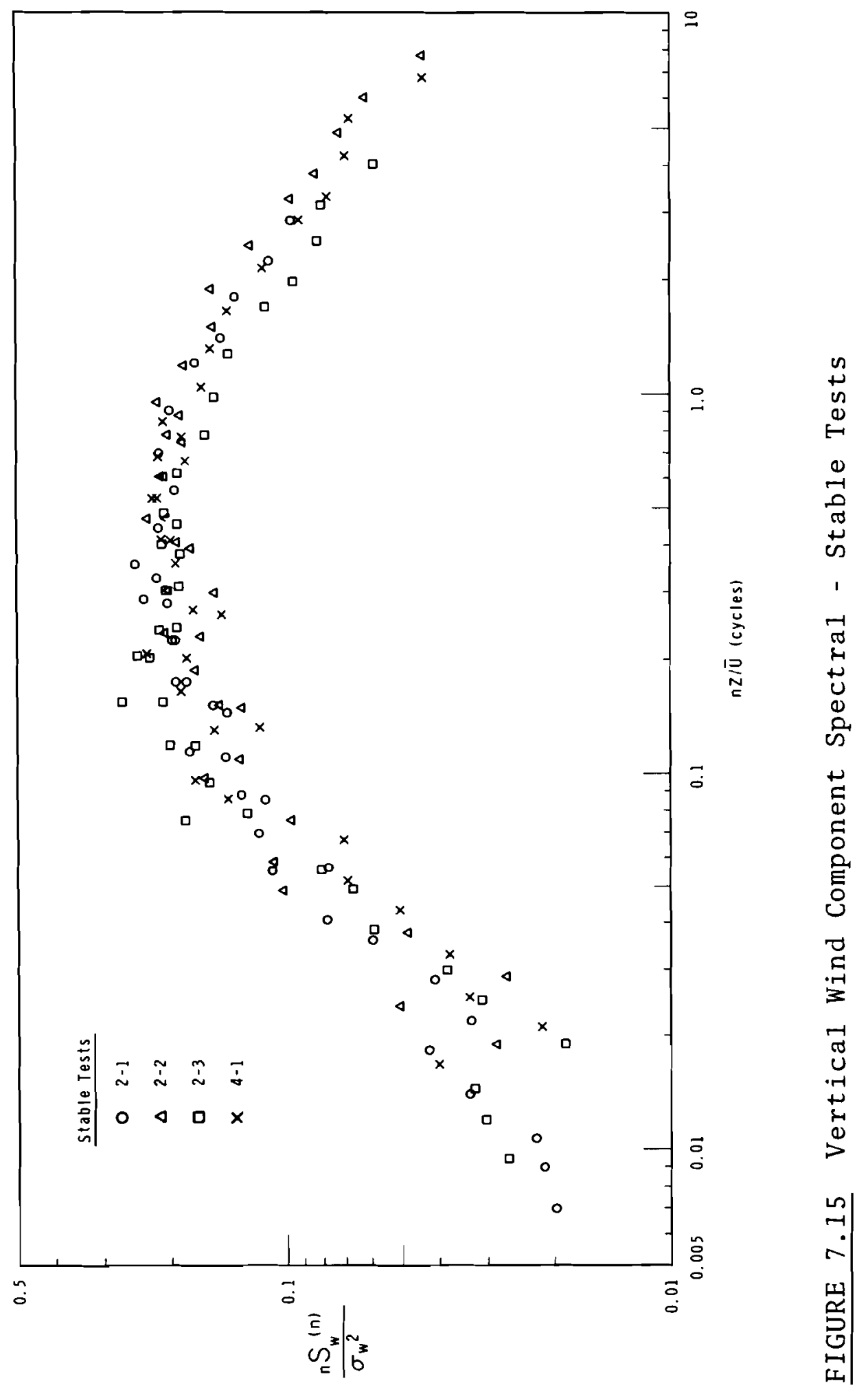




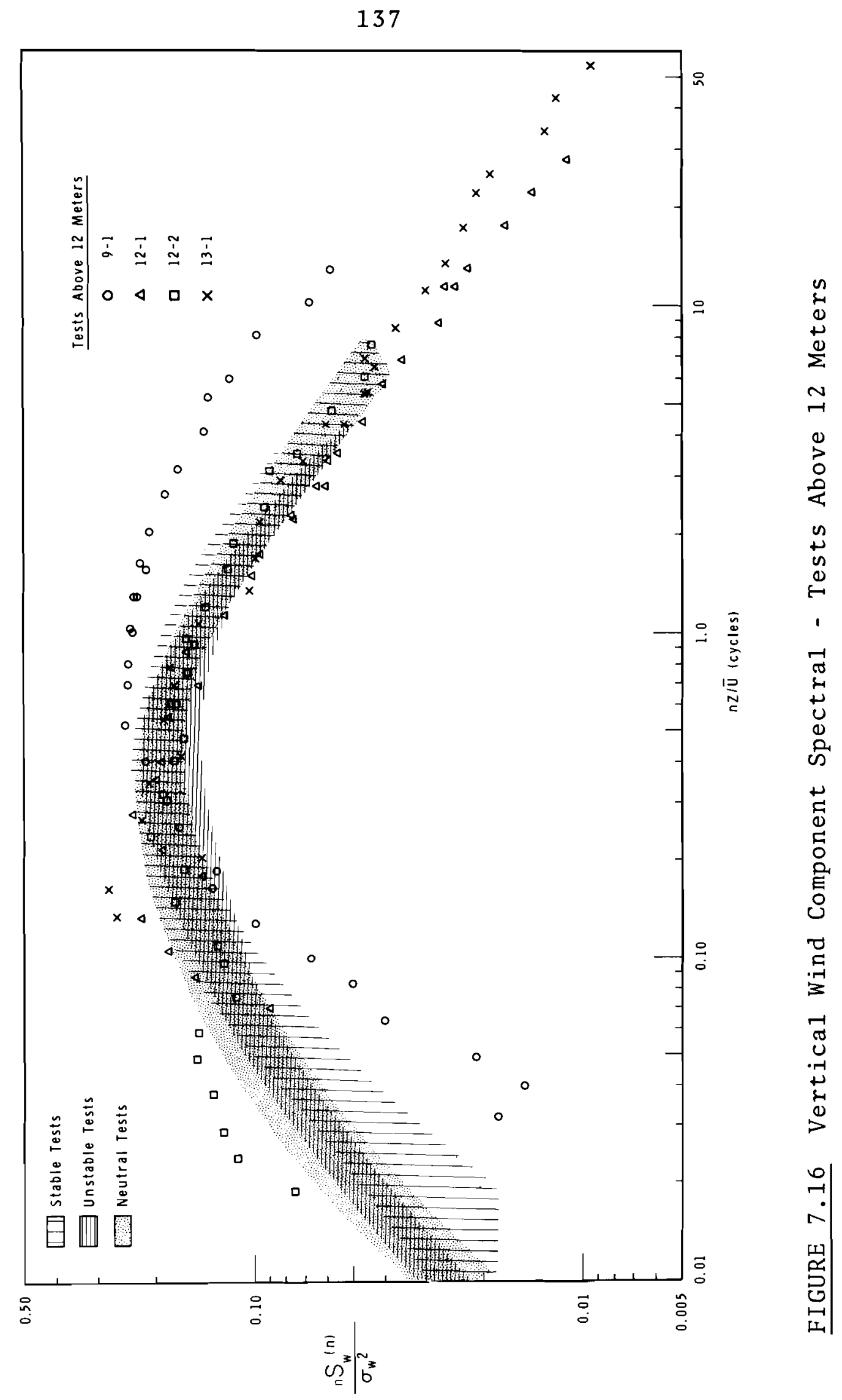




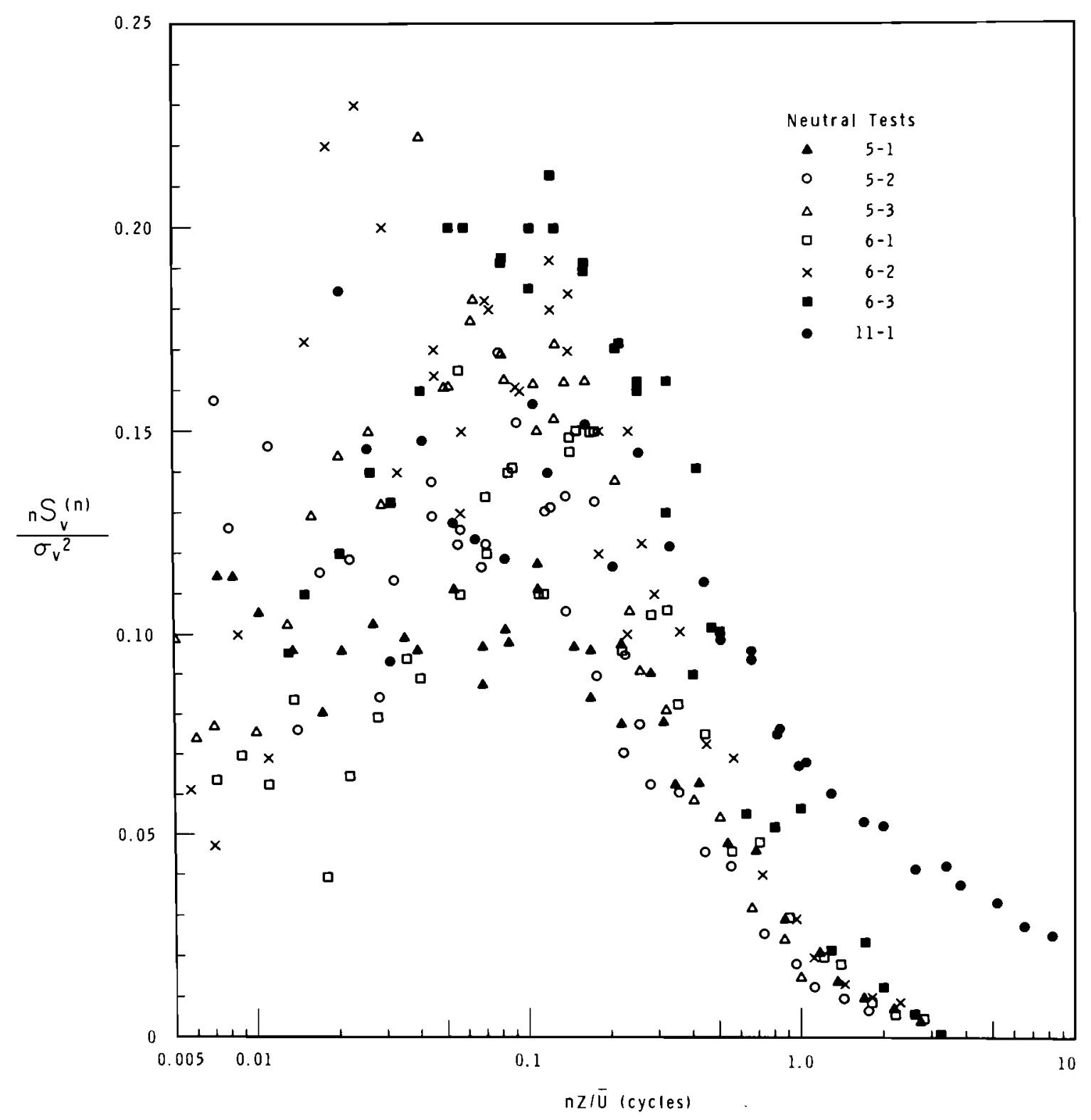

FIGURE 7.17 Latera1 Wind Component Spectra - Natural Tests 


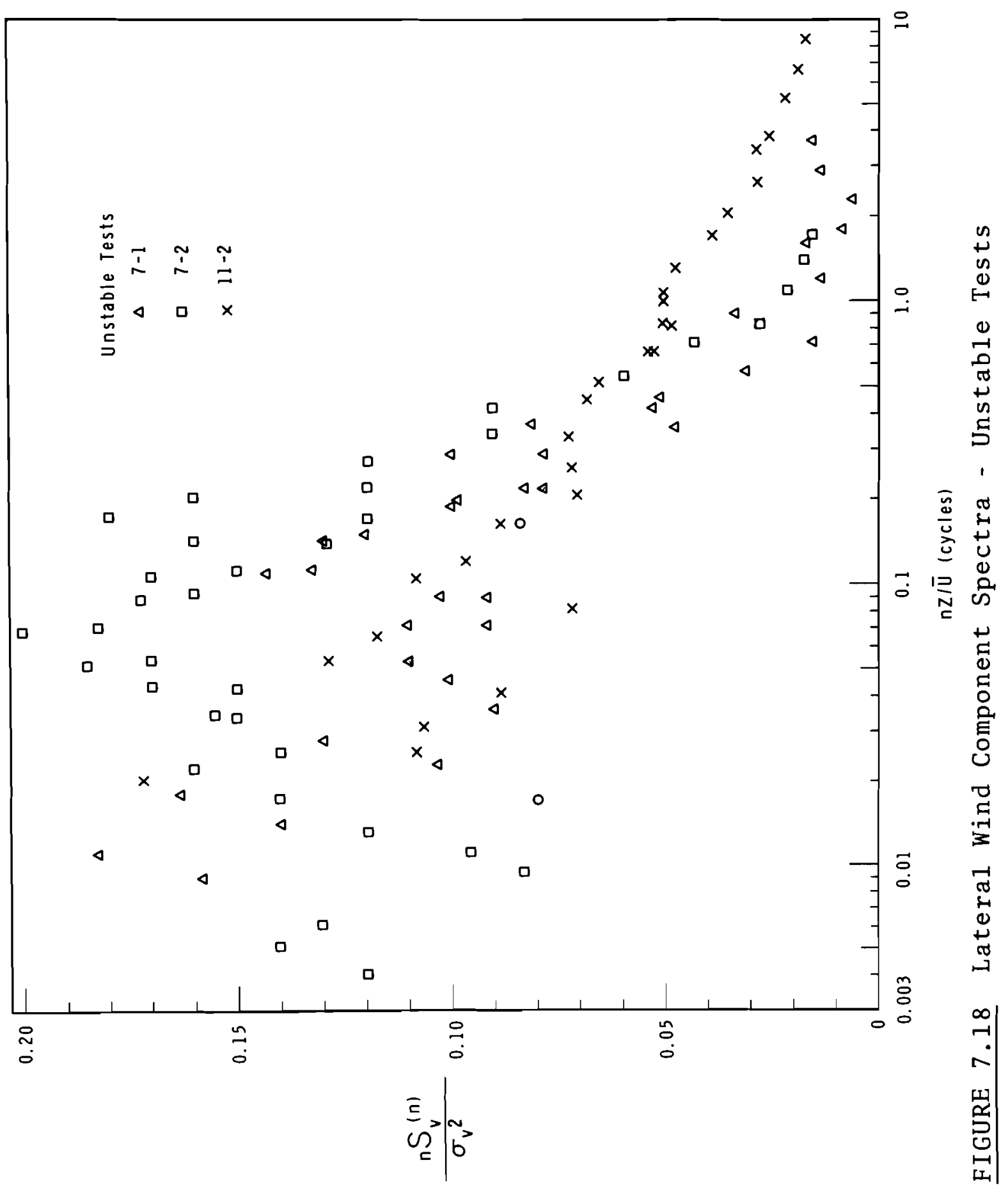




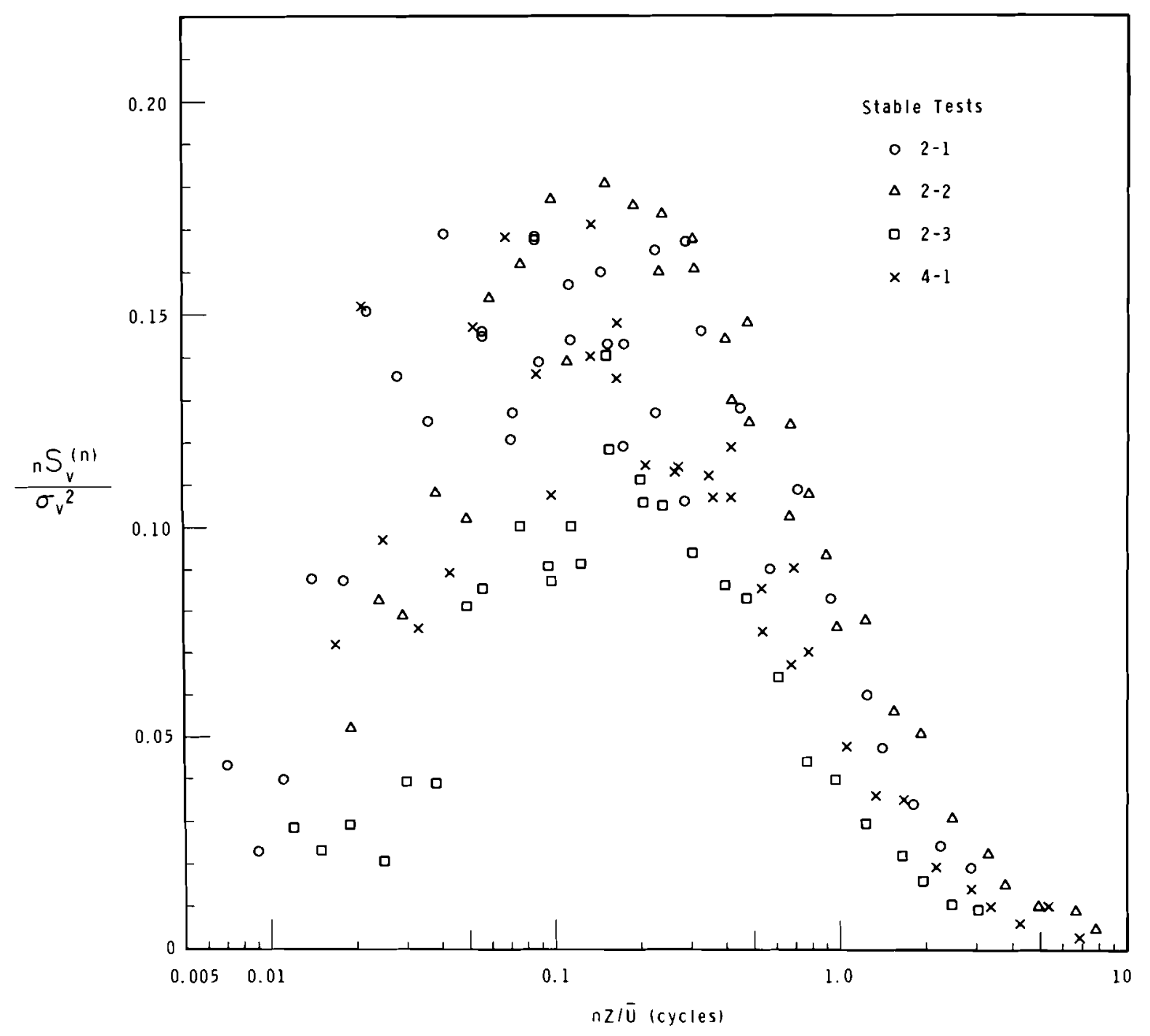

FIGURE 7.19 Latera1 Wind Component Spectra - Stable Tests 


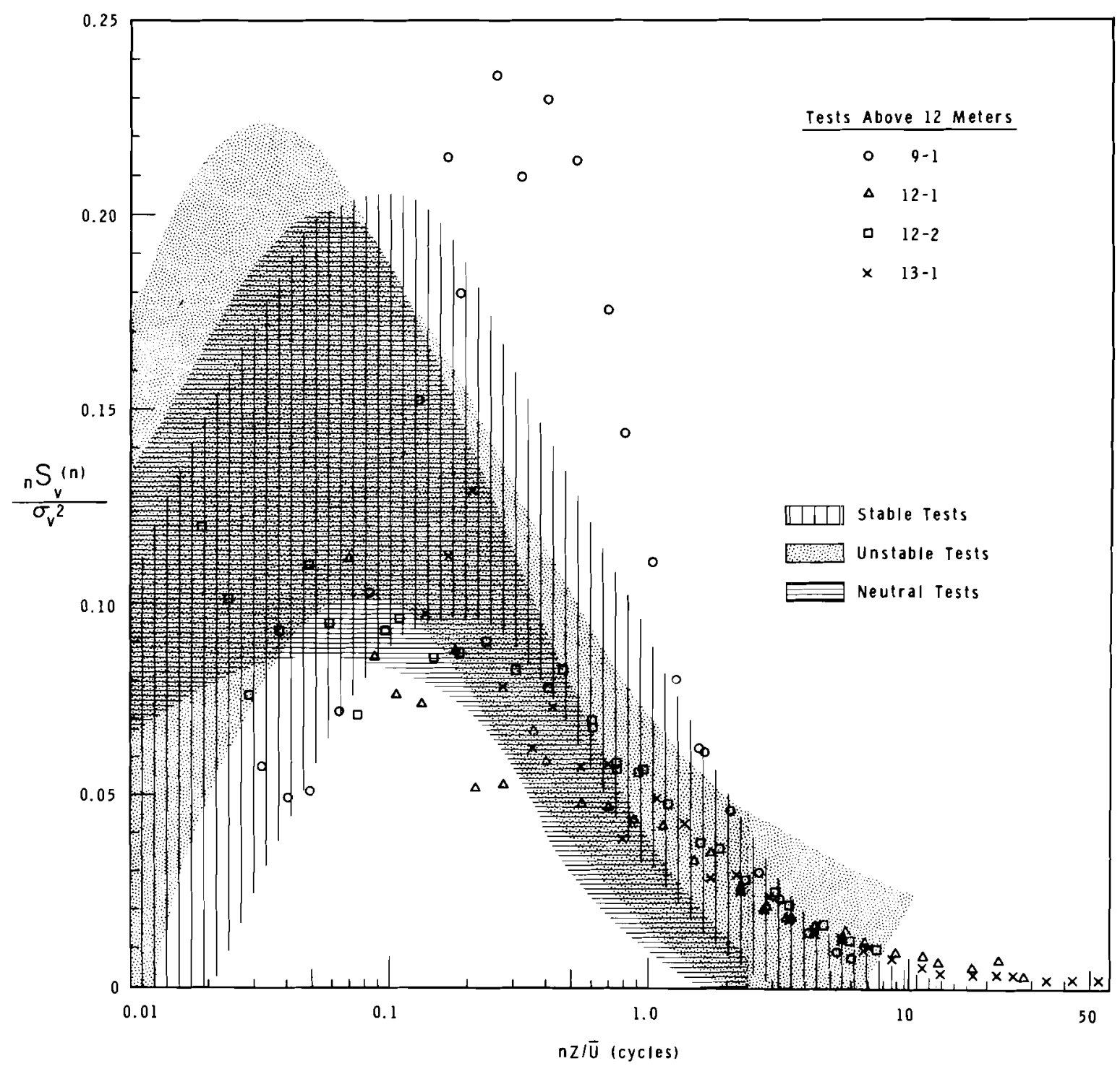

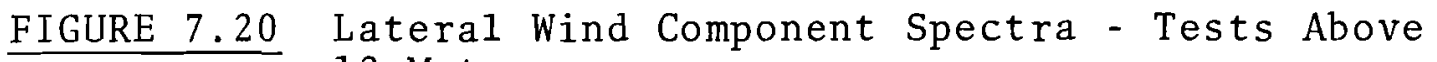
12 Meters 


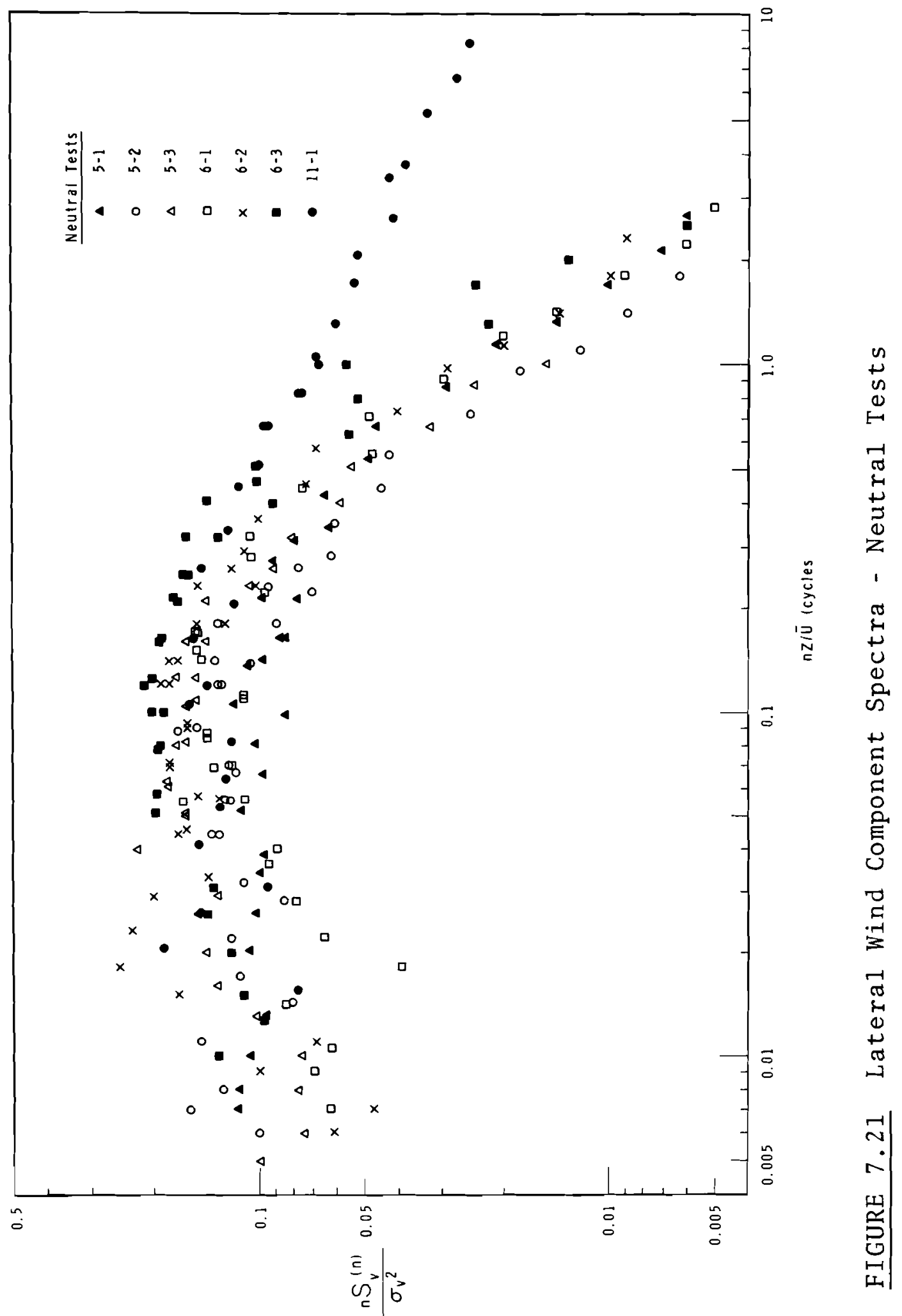




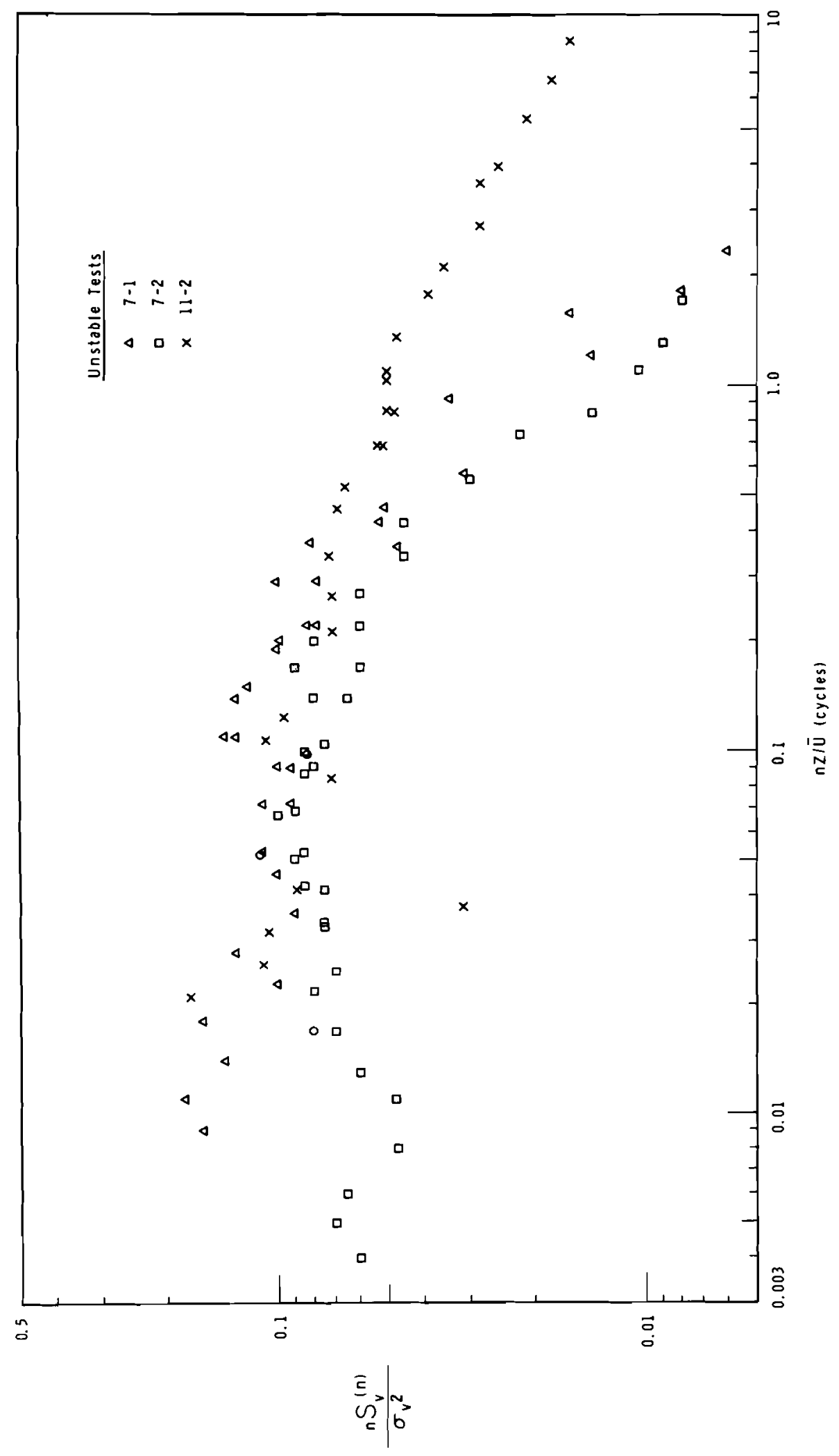

O

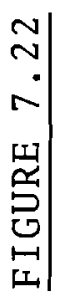




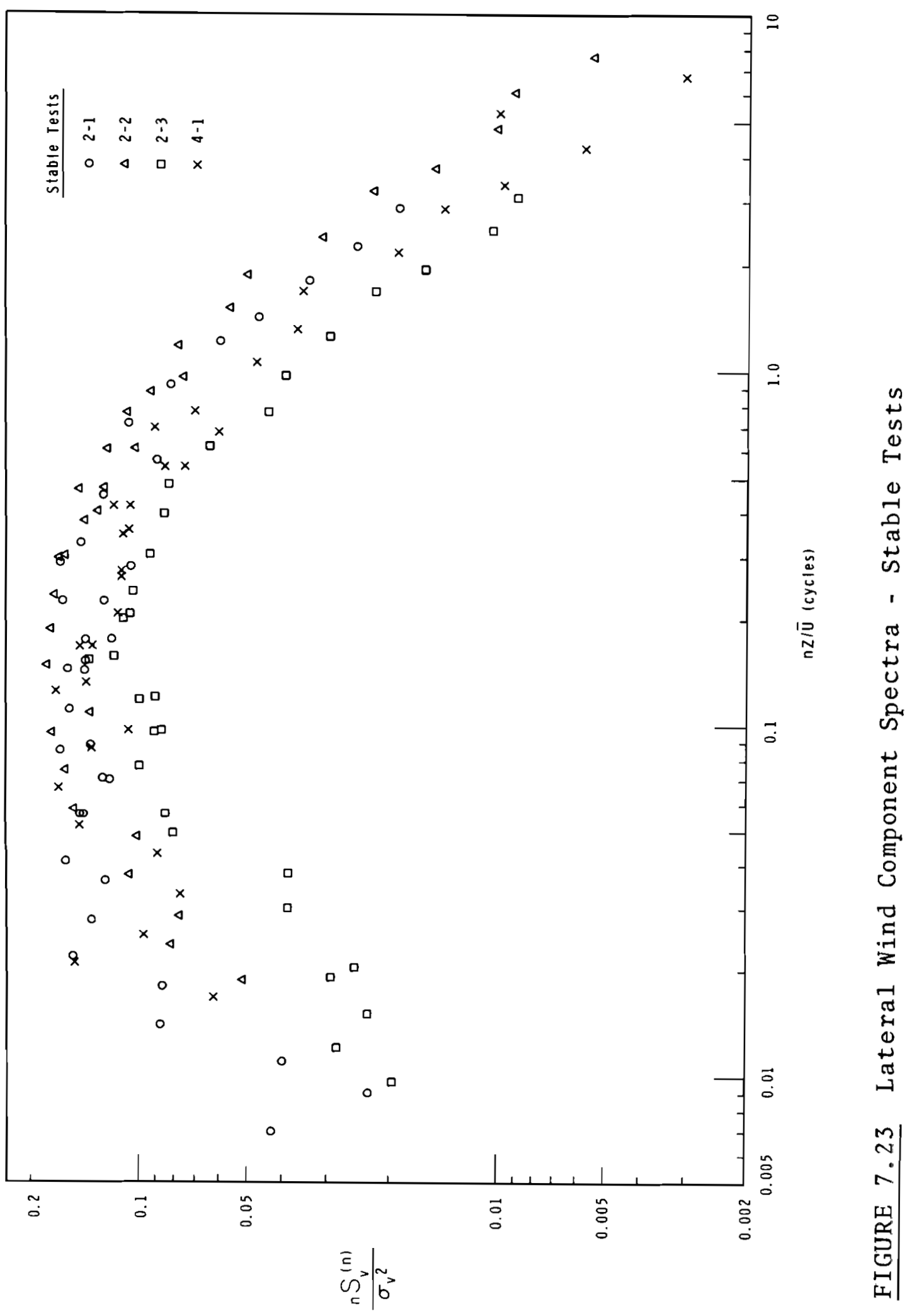




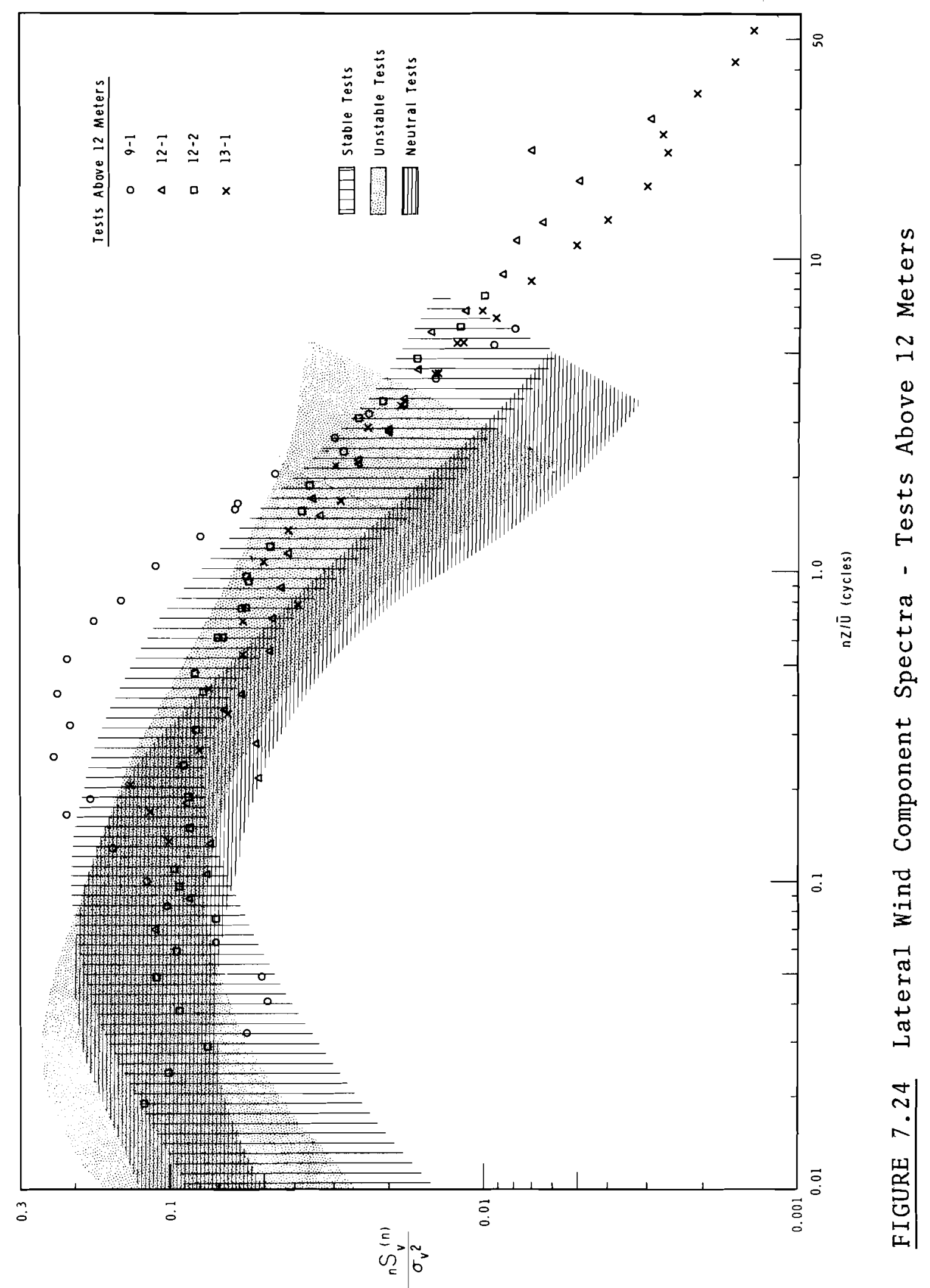




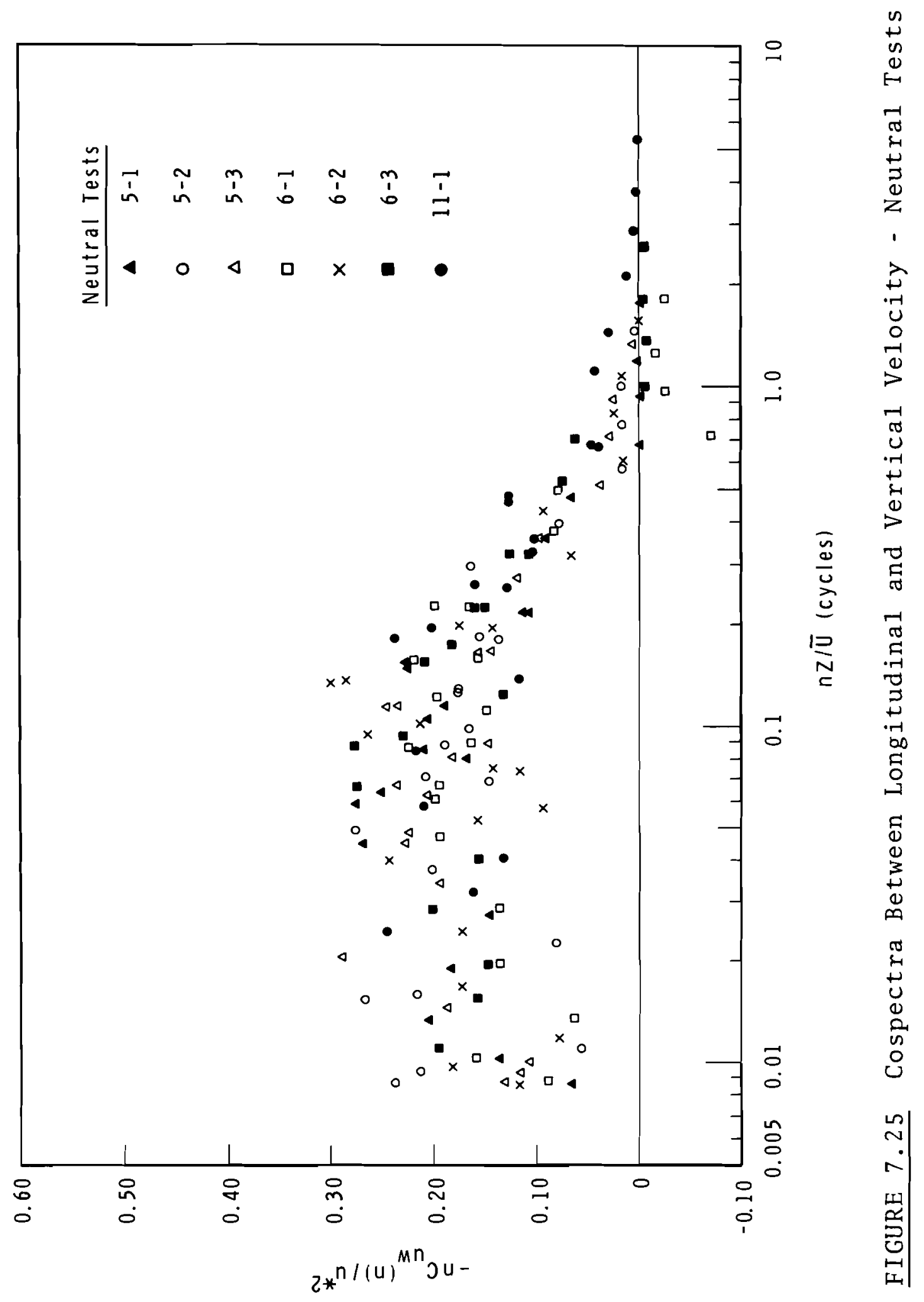




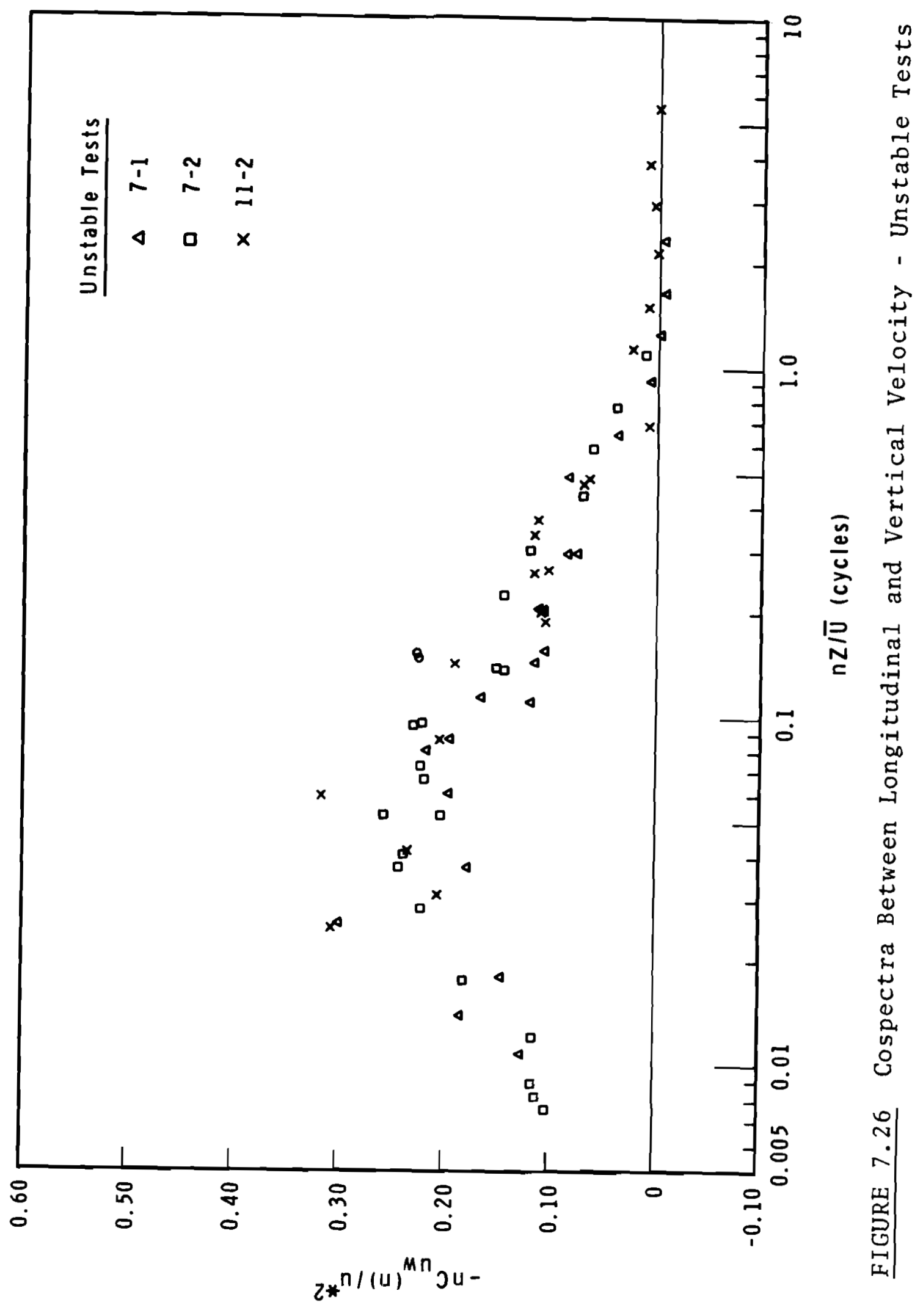




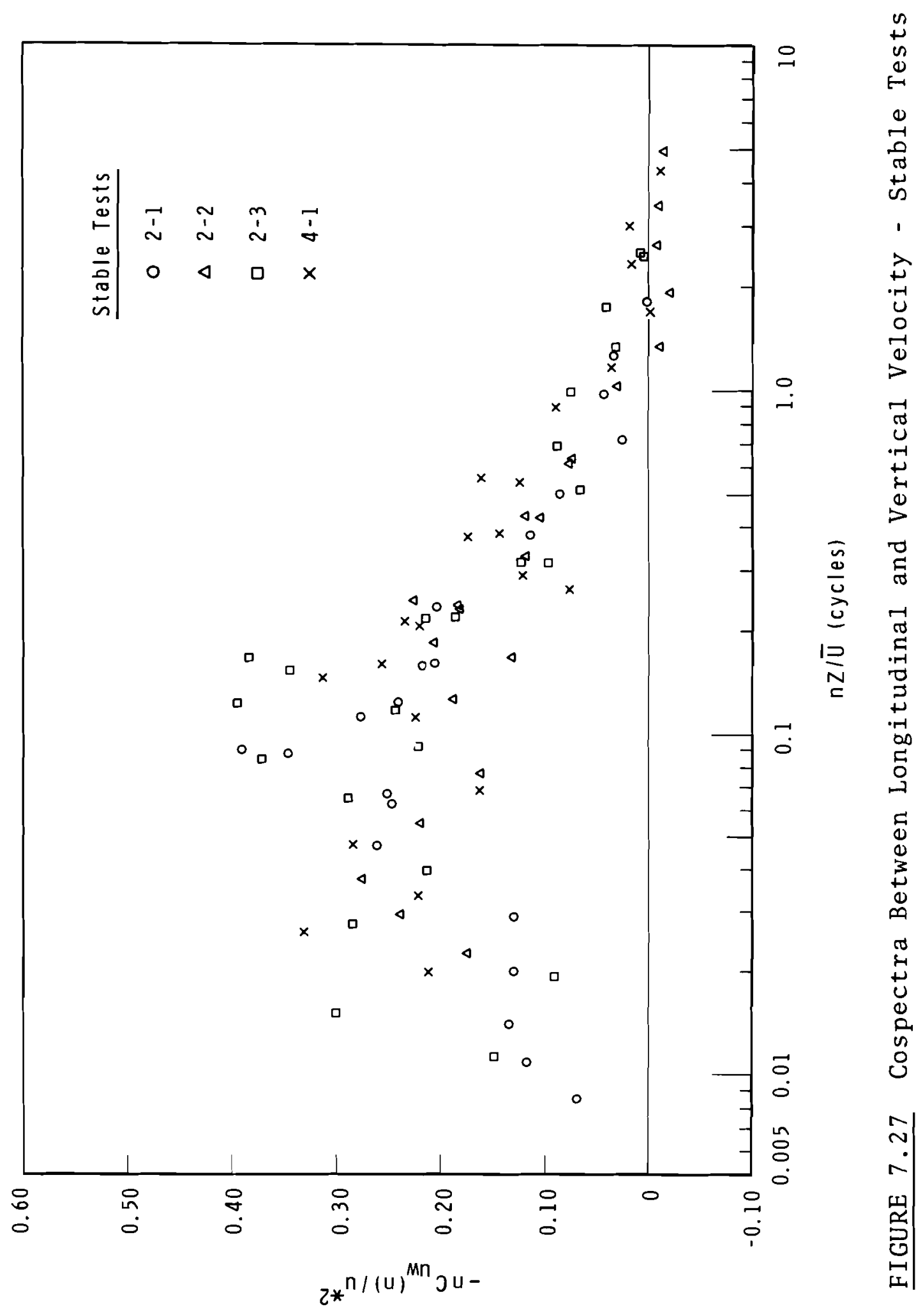




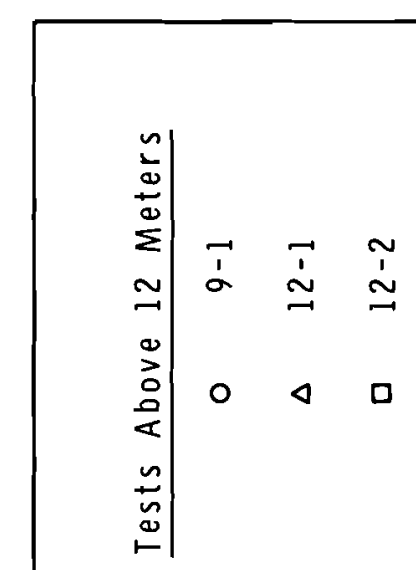

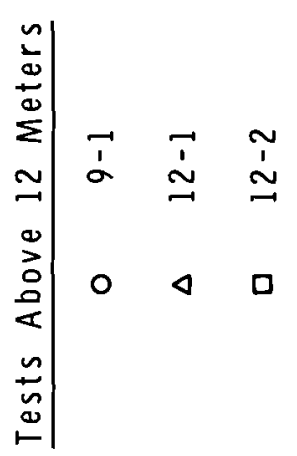

4

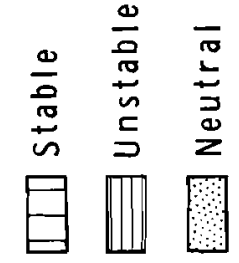

$\triangleleft$
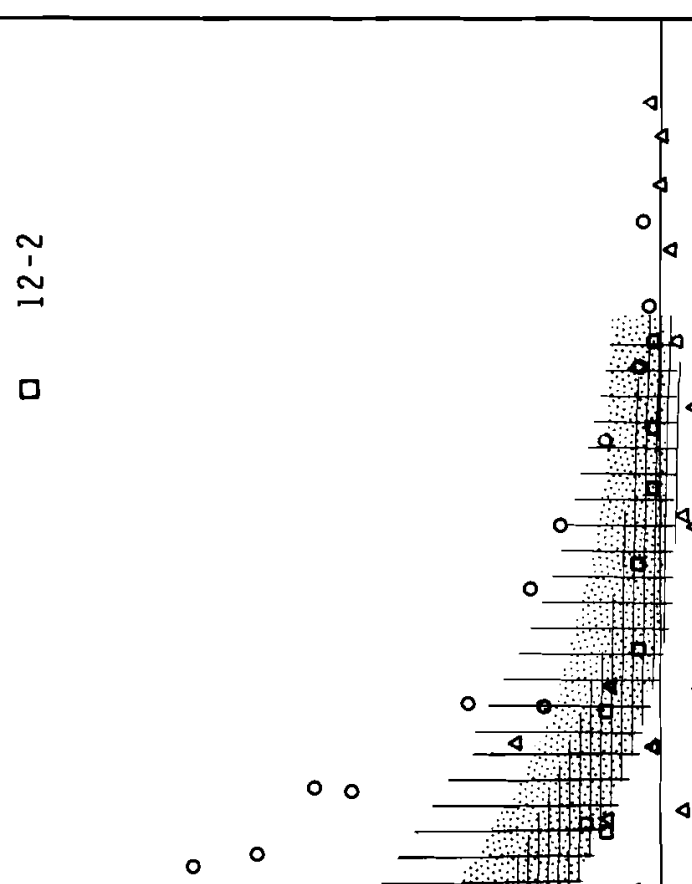

ㅇำ<smiles>C1CCCCC1</smiles>

o

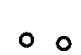

$\circ 。$

$\circ$
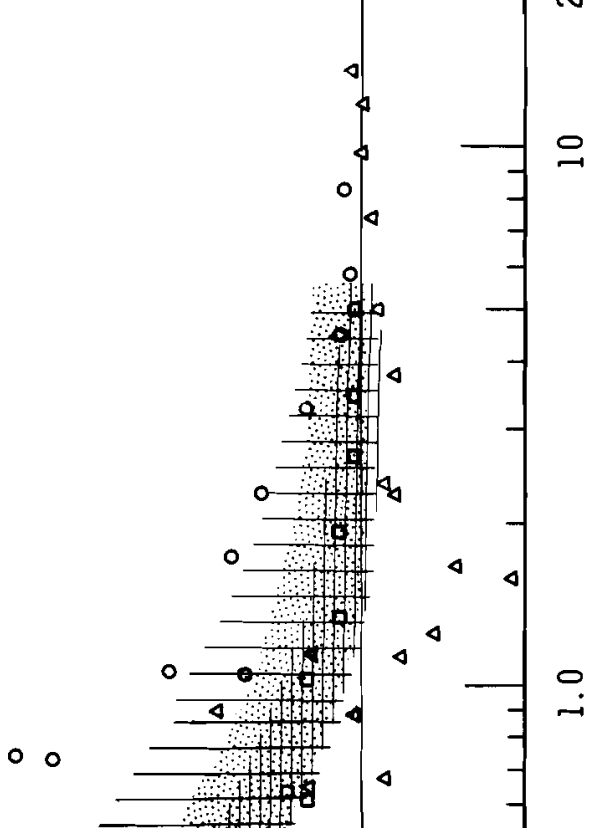

0
0
0
0
1
0
1
0
0
-1

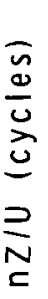

0

$\circ$
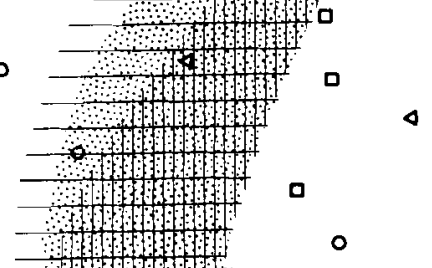

$+4$

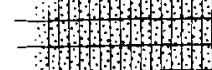

$+(4)$

$-4(4)$

4 H

$+4$

H

4

$+4.9$

+4 H.

$\circ$

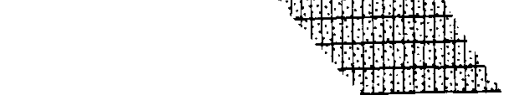

$\circ$ 


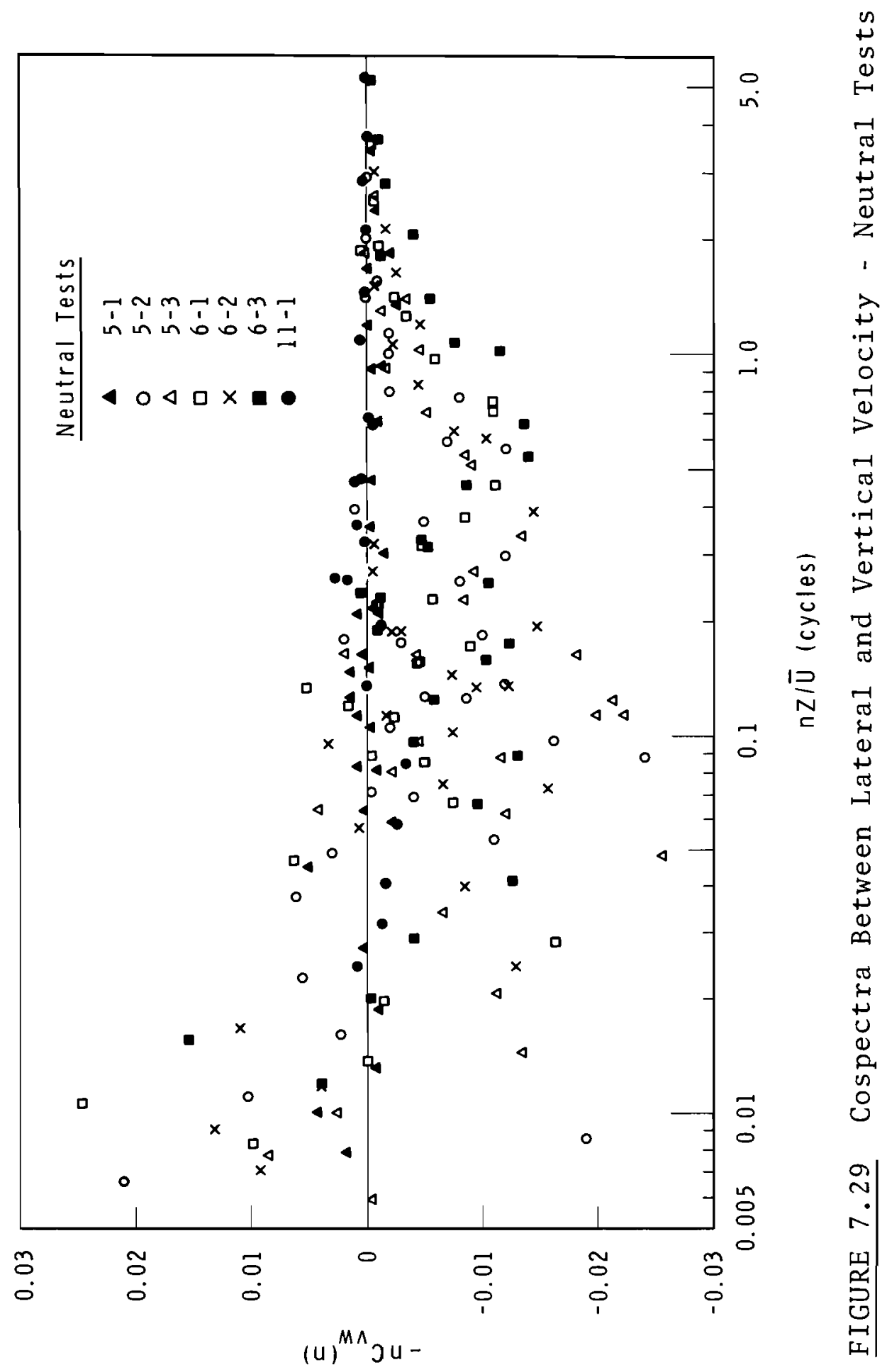




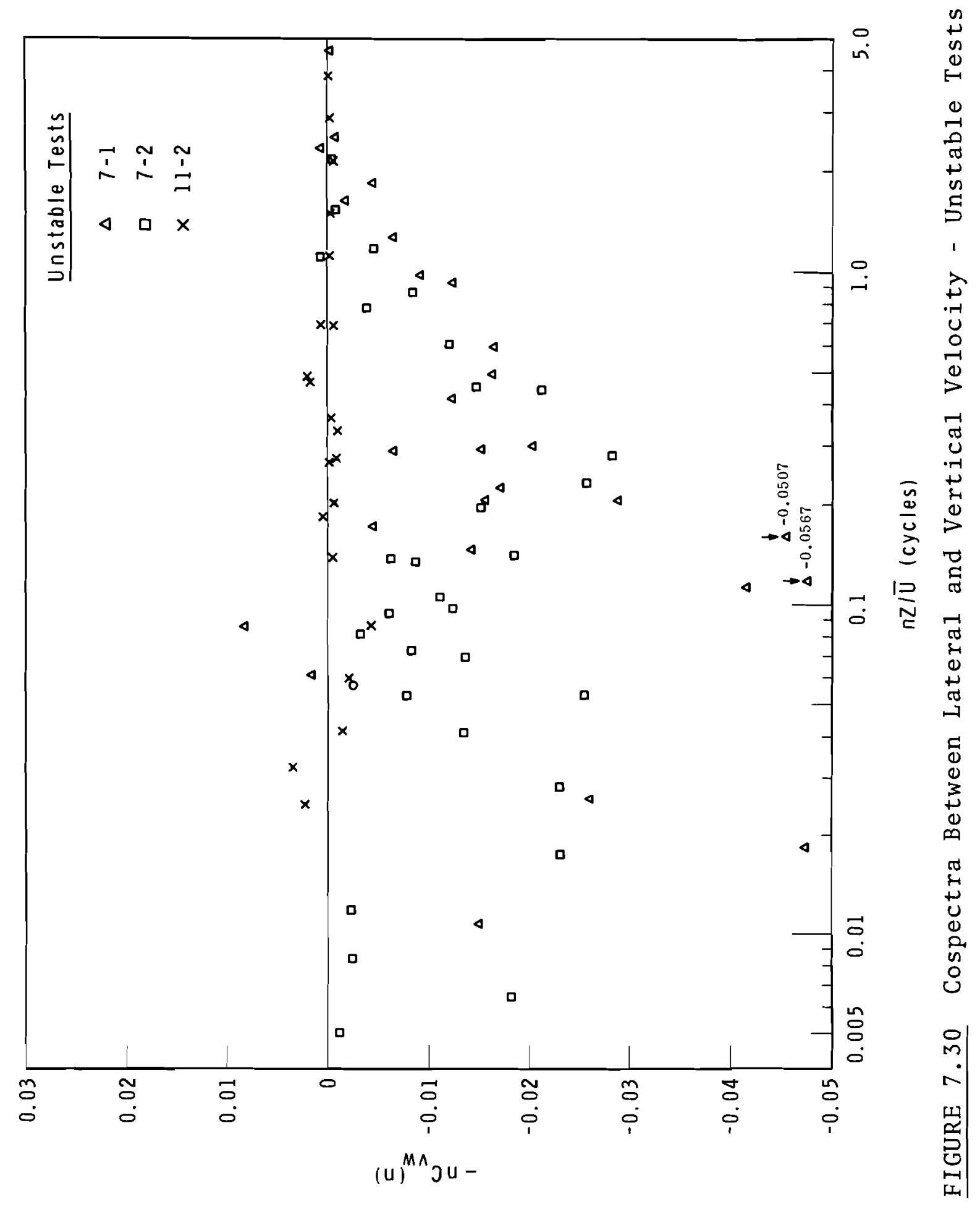




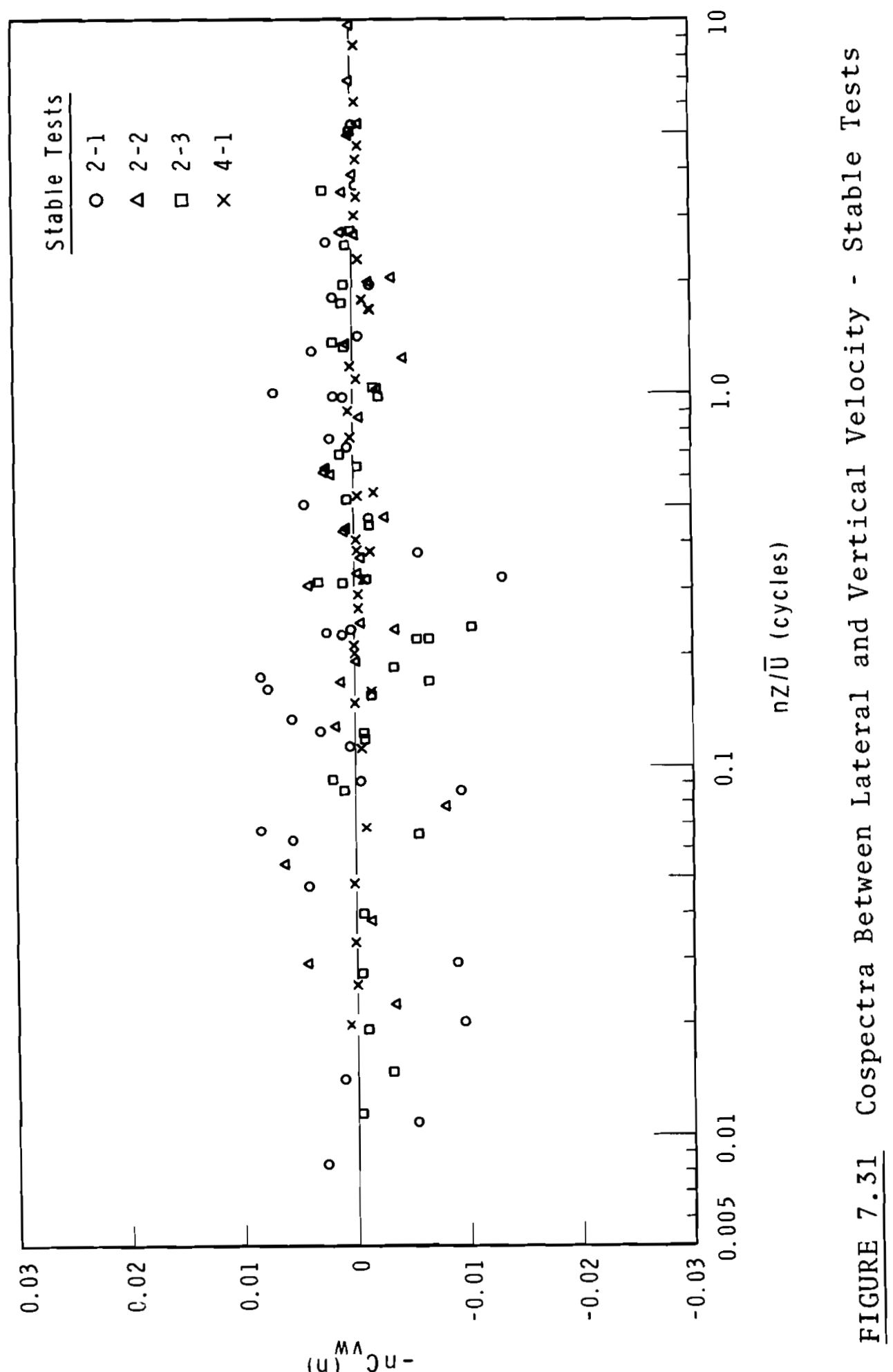




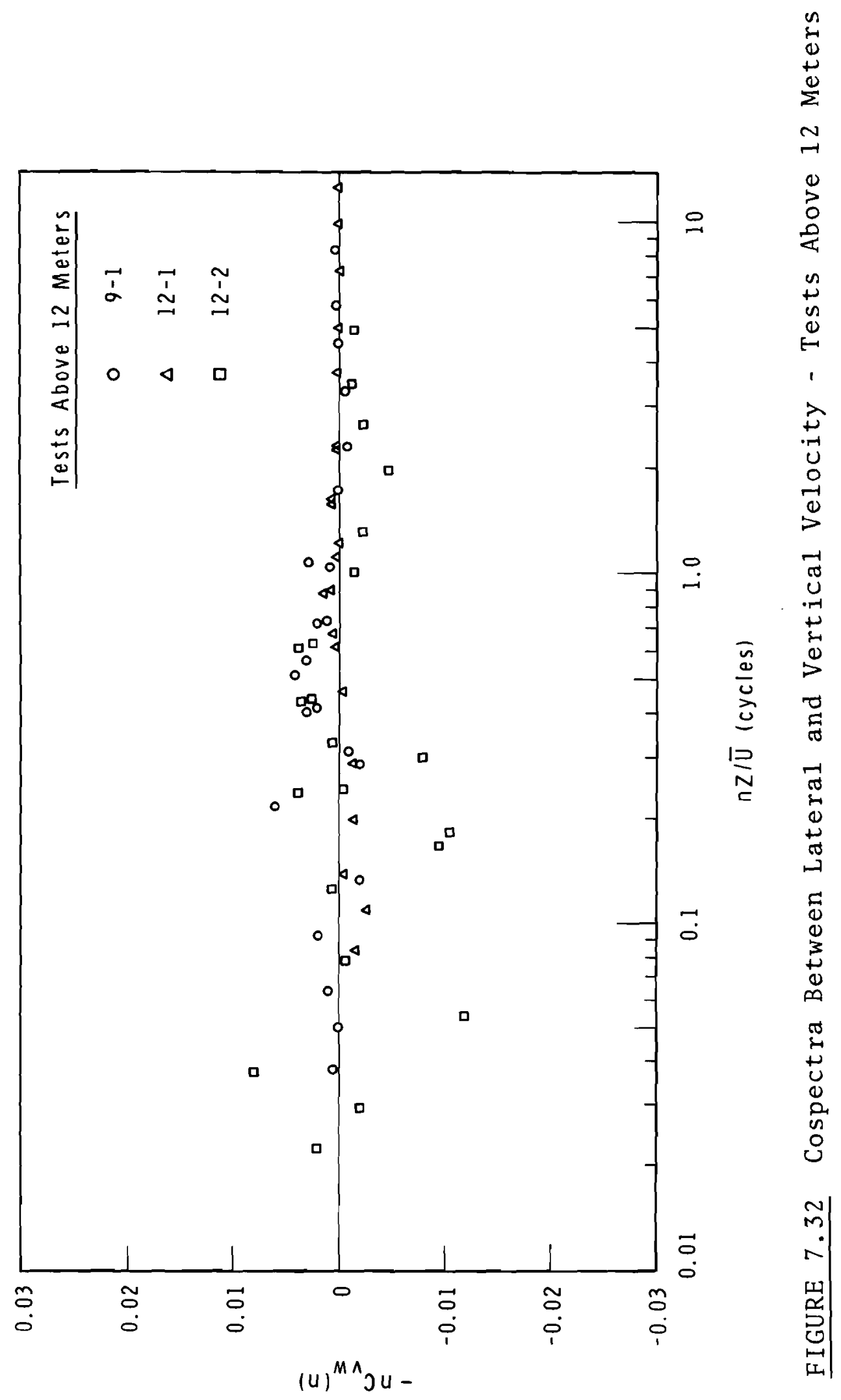




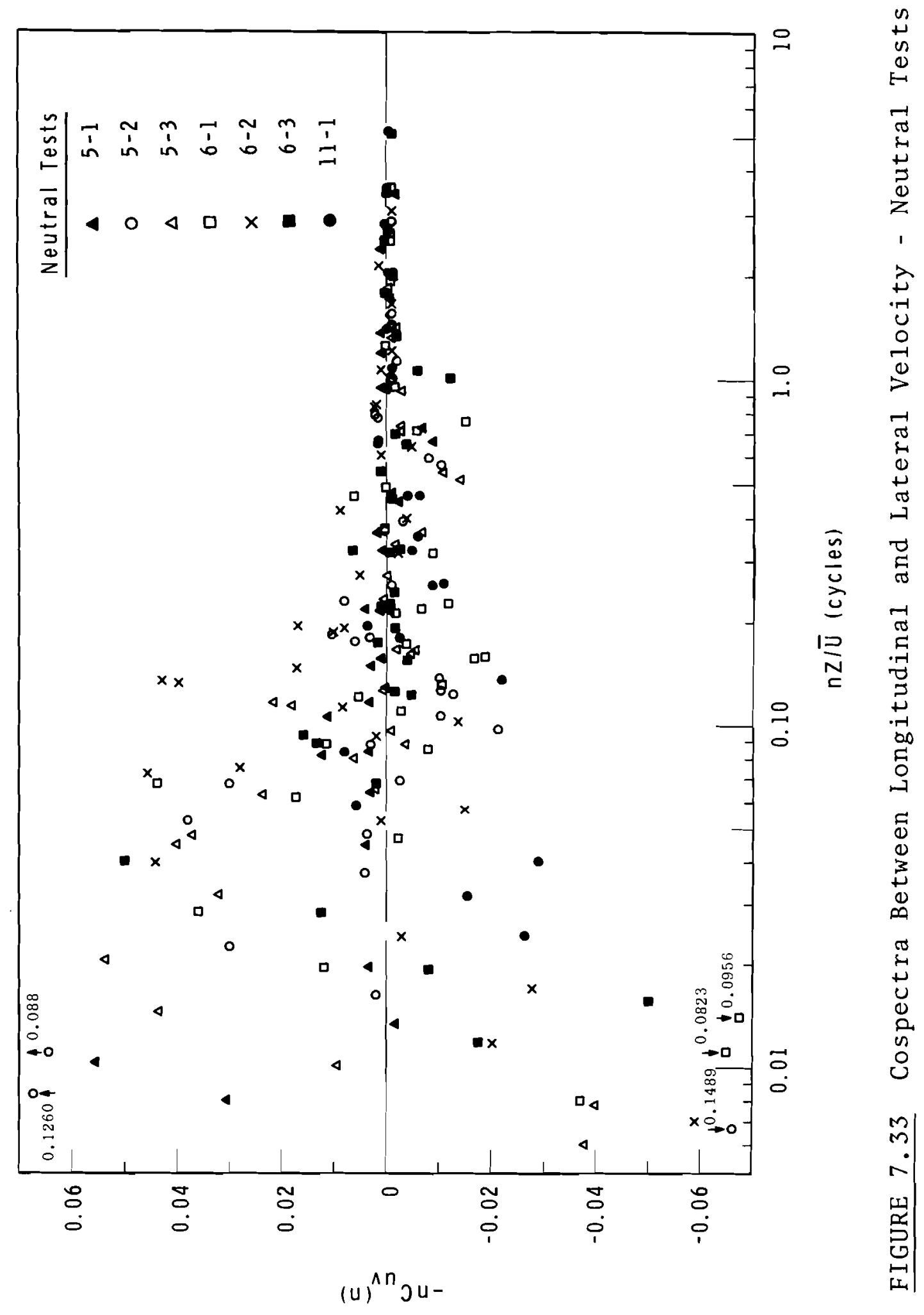




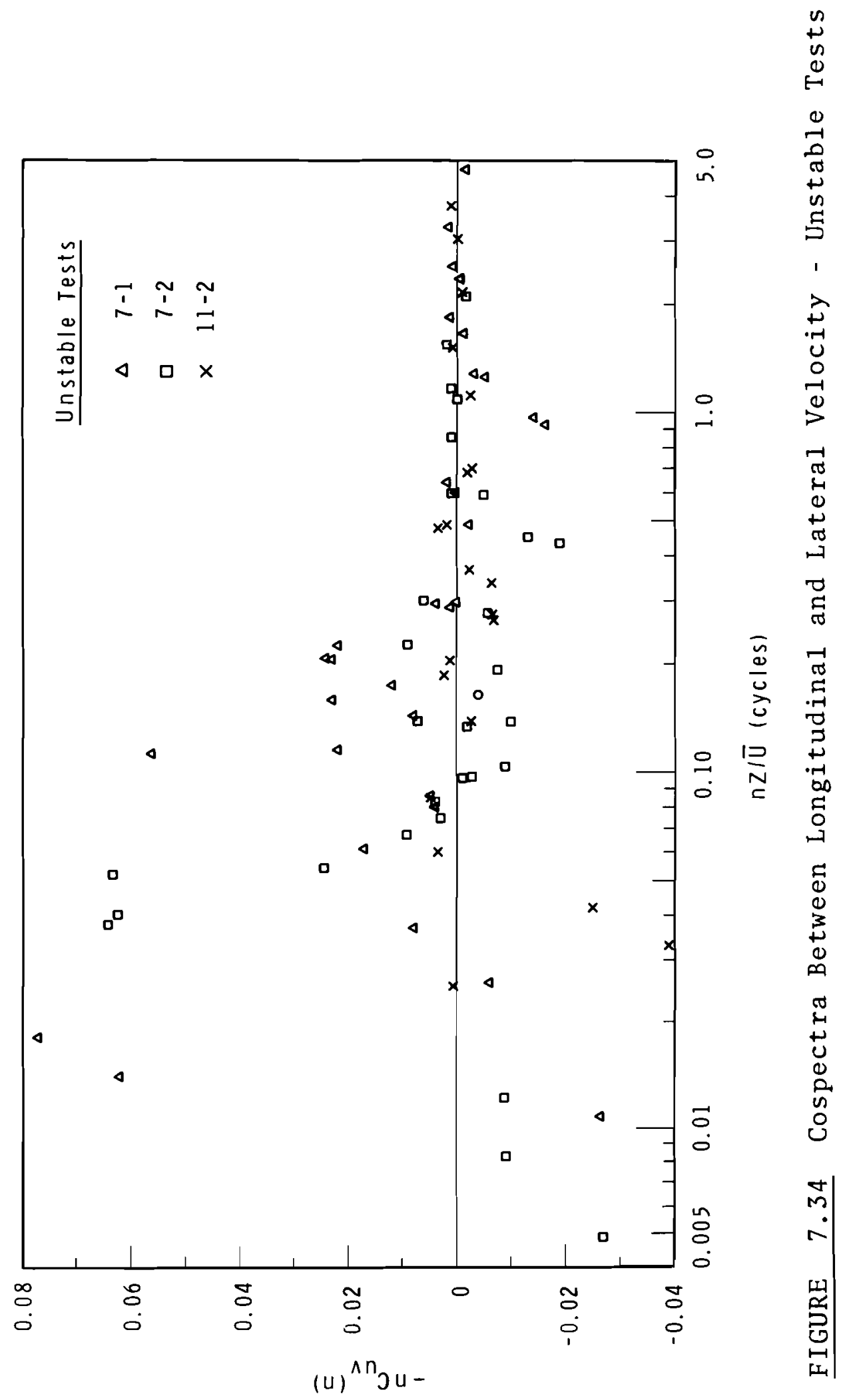




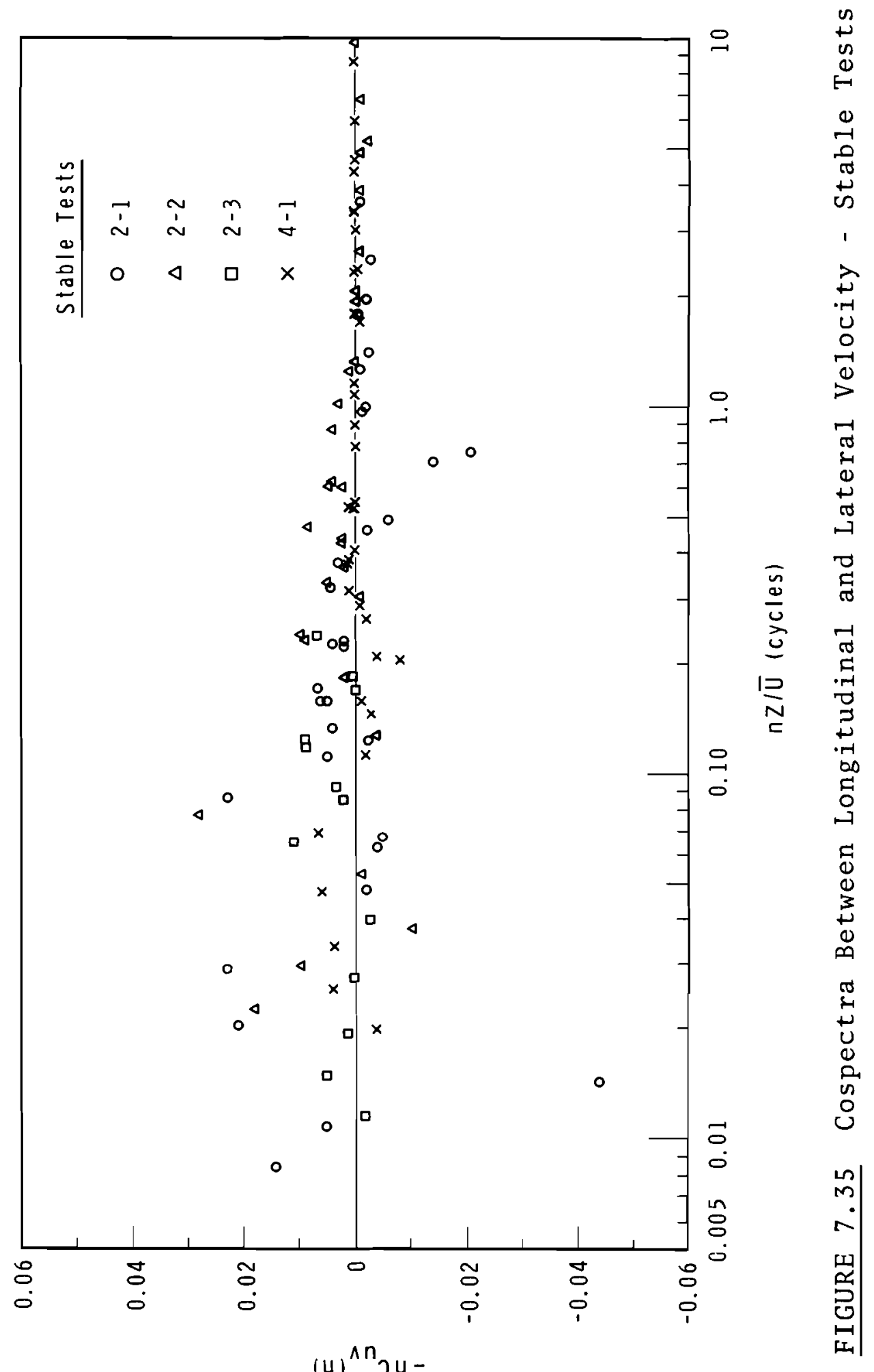




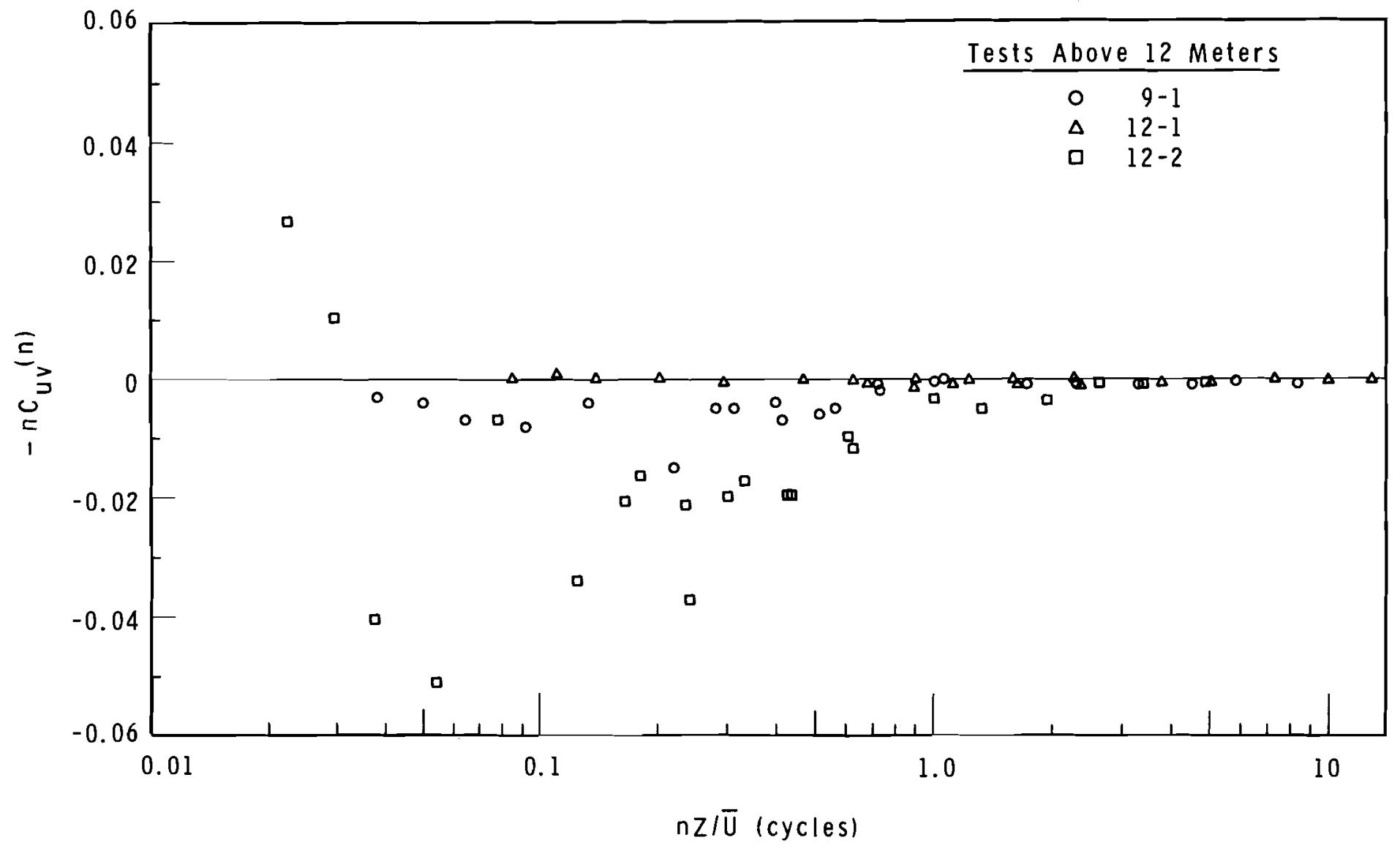

$\underline{\text { FIGURE } 7.36}$ Cospectra Between Longitudinal and Lateral Velocity - Tests Above 


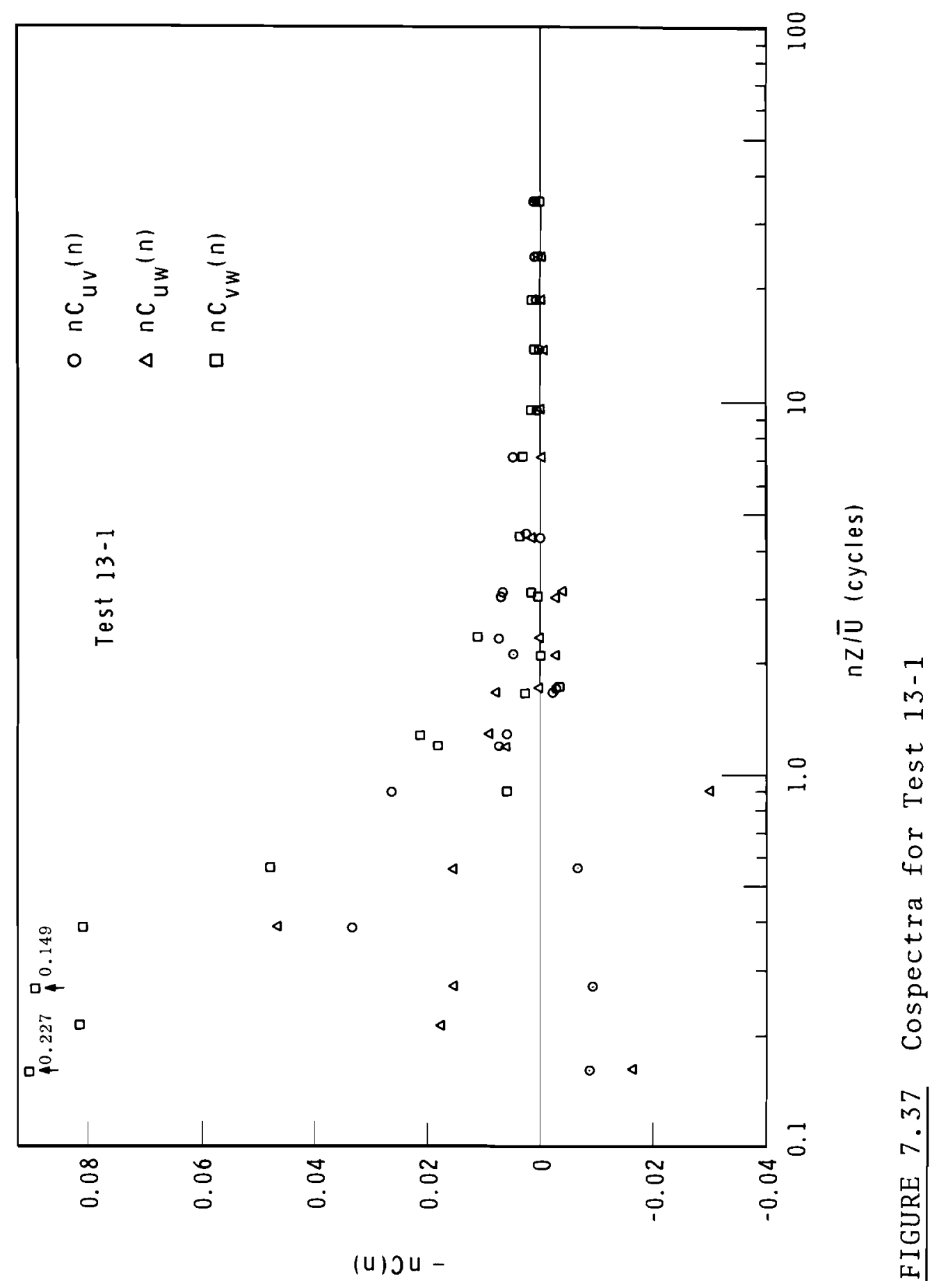




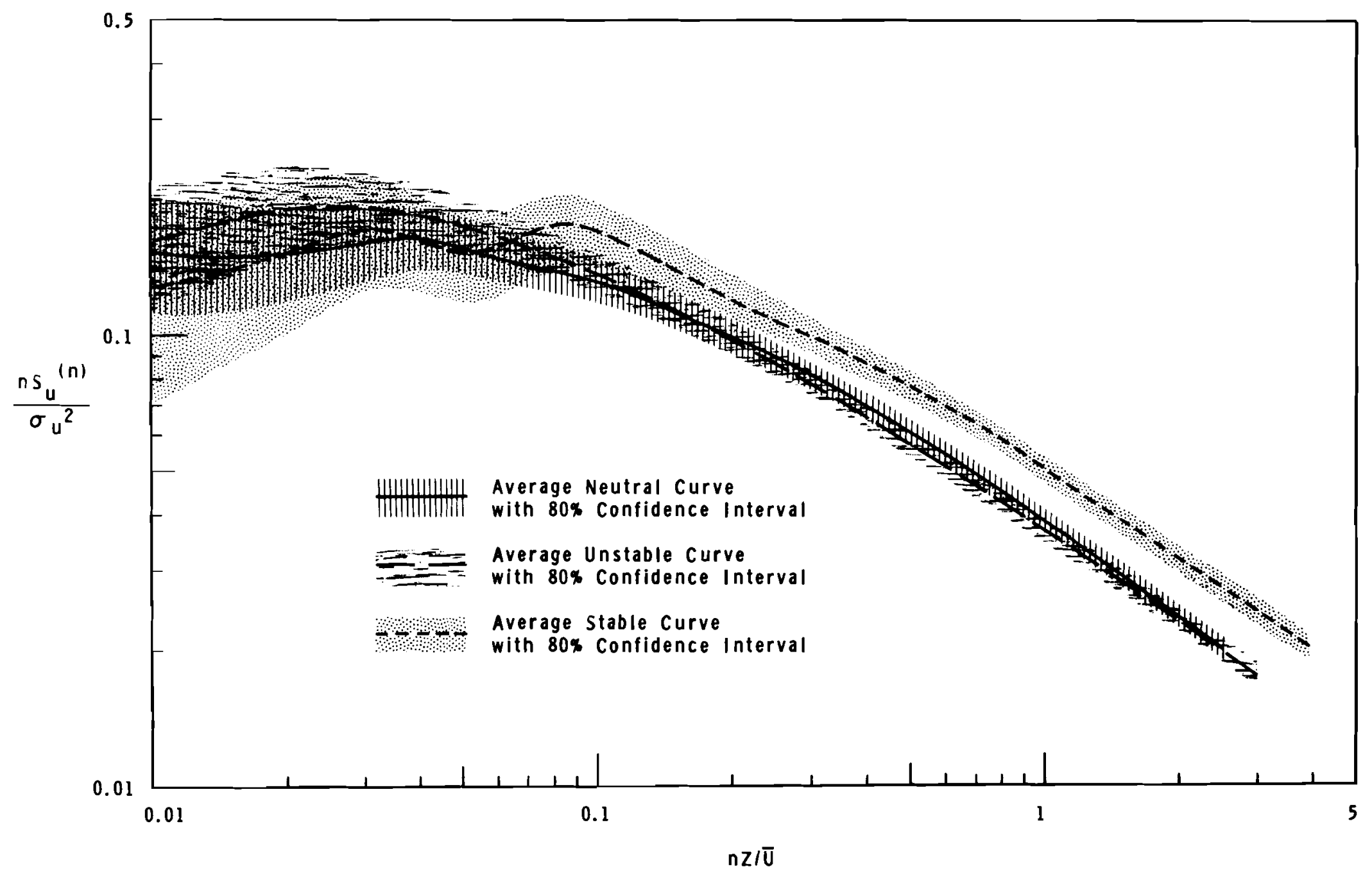

FIGURE 7.38 Average Longitudinal Wind Component Spectrum 


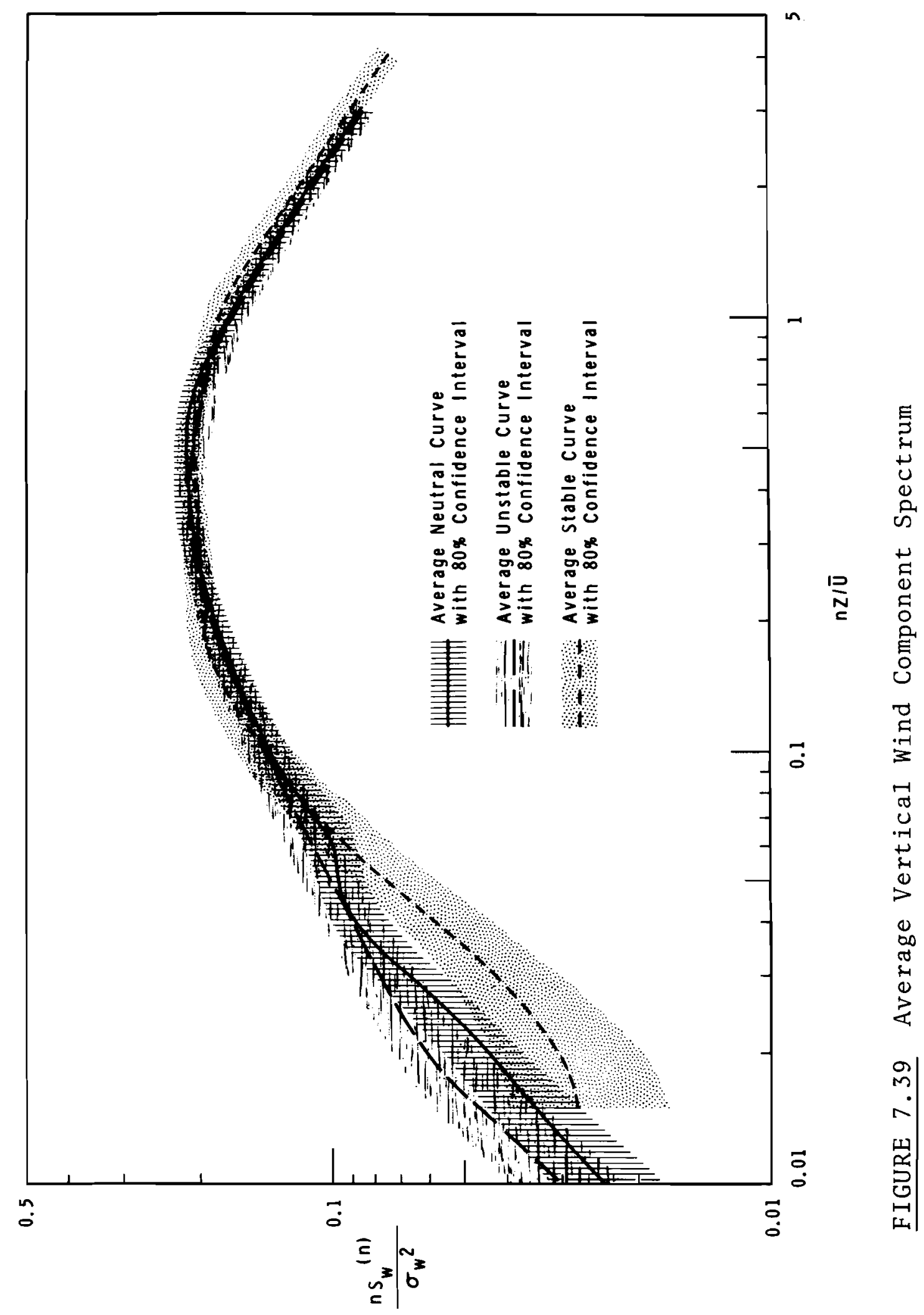




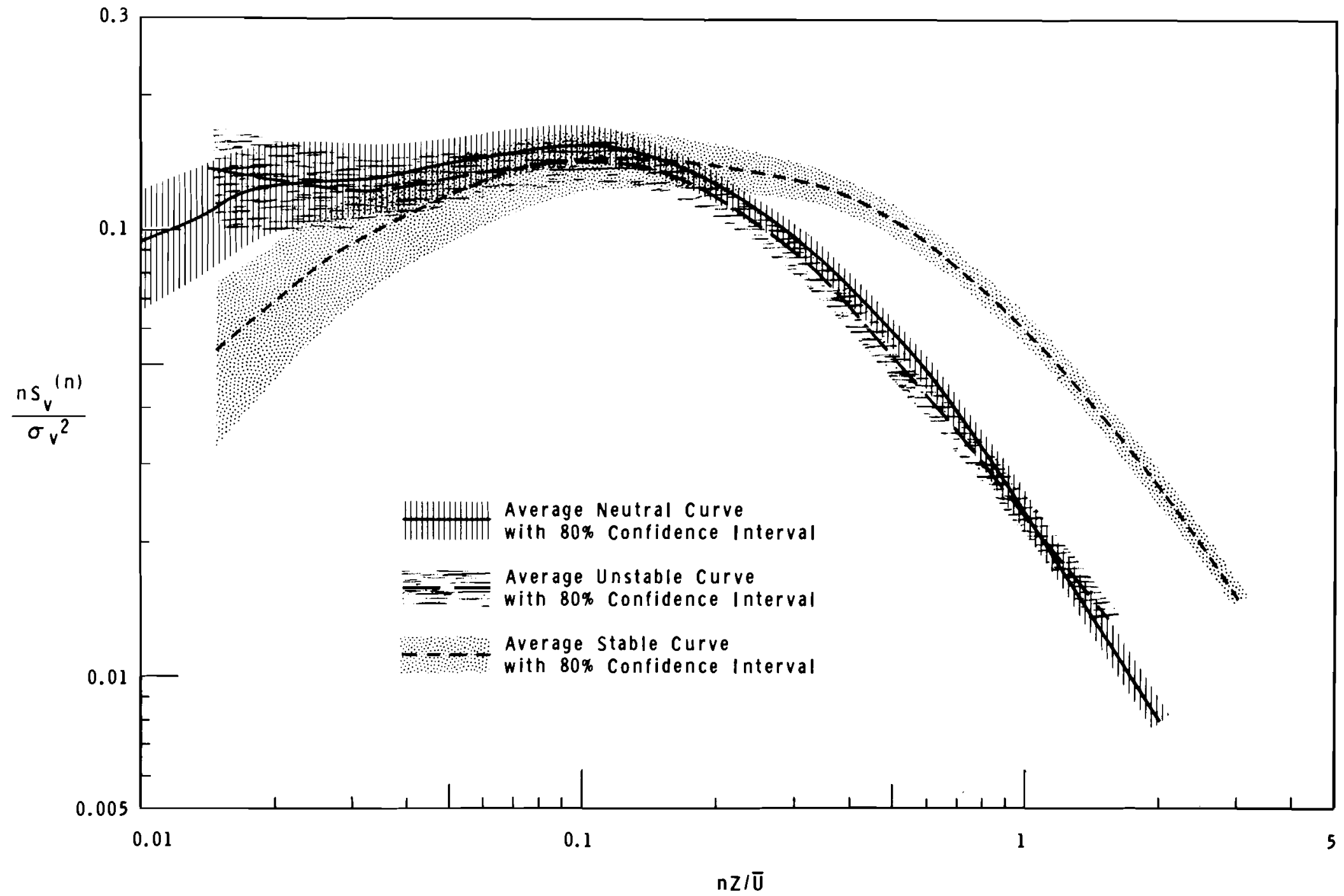




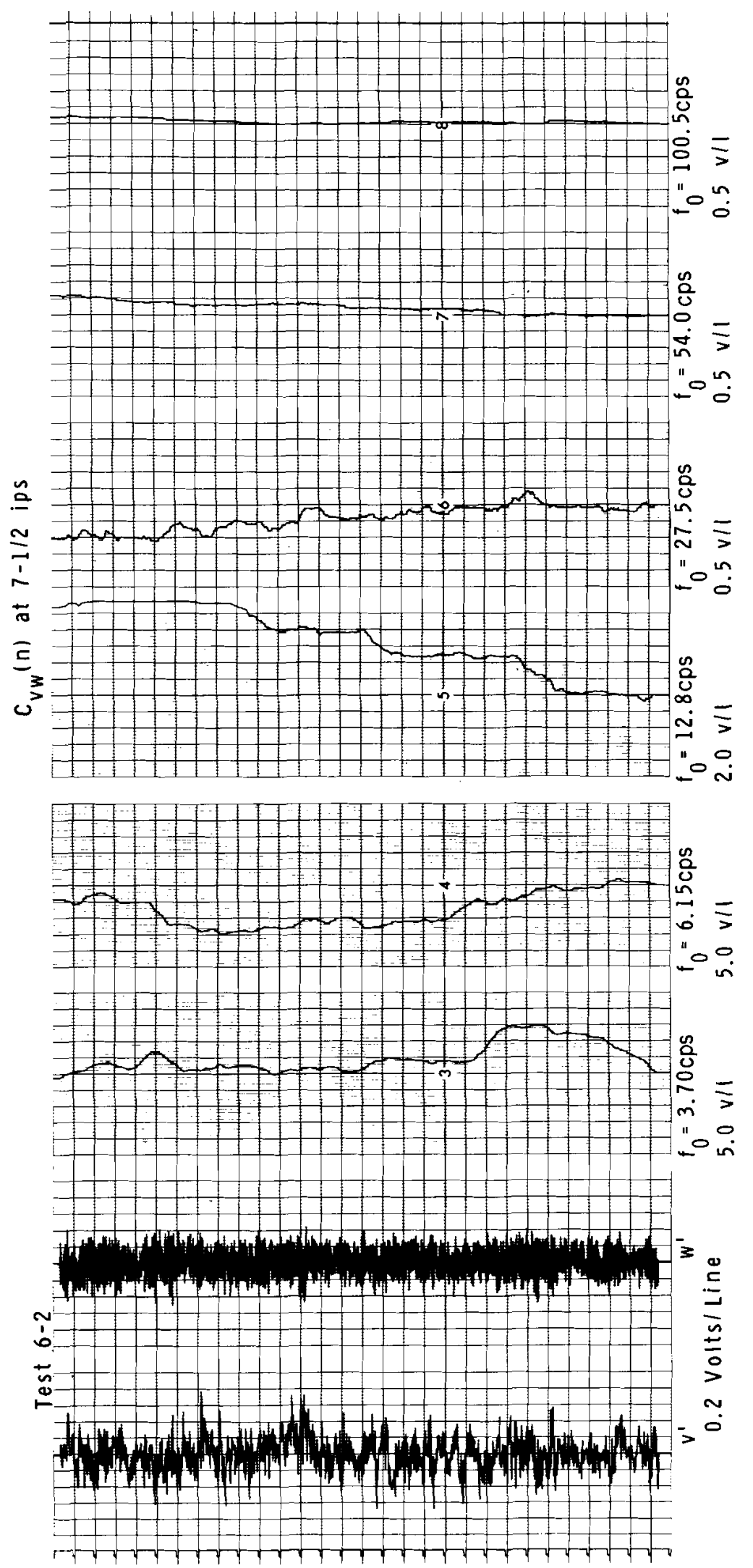

5
0
-1
0
0
7
3
0
7
0
0
7
0
0
0
0
0
0
0
0
0 


\section{SUMMARY AND CONCLUSIONS}

Turbulence data has been analyzed for power spectral distributions, cospectral distributions and Reynolds' stresses using an analog computer. One of the most significant results found in the study was the degree of similarity shown between the power spectral distributions for any given component as well as for the cospectral distributions of $\overline{u^{\prime} w^{\prime}}$. Similarity of spectral distributions is most obvious and consistent for the vertical component with only a few deviations. Measurements at 3.0 and 6.1 meters yield the same distribution of normalized spectral energy, $\mathrm{nS}_{w}(n) / \sigma_{w}{ }^{2}$, as a function of normalized wave number, $n z / \bar{U}$, for neutral and unstable conditions with only one unstable case at 6.1 meters suggesting the presence of convective energy at low wave numbers. The unstable tests at 12.2 and 87 meters, however, while corresponding to the similar shape at high wave numbers including the mechanical energy peak, demonstrate a significant departure due to the convective energy input at low frequencies, revealing a convective energy peak not clearly identified previously. The stable tests show over the entire wave number range only a slight shift to higher $n z / \bar{U}$ values at 3.0 and 6.1 meters but at 12.2 meters a very obvious shift to high wave numbers demonstrates that the eddy sizes are scaled not simply according to height but also by stability. 
although at low wave numbers increased variability is noted. Again, the stable cases show only a slight shift to higher wave numbers for the low level tests but a large shift is observed for the 12.2 meter test. The peaks in the longitudinal spectra occur generally at $n z / \bar{U}=0.03$, much lower than the vertical component spectral peaks near $n z / \bar{U}=0.4$. The lateral component spectra also show some similarity, particularly above $n z / \bar{U}=0.1$. Below this point, however, a great deal of variability is observed. The stable tests exhibit the best similarity and, as with the other components, are shifted to somewhat higher $\mathrm{nz} / \overline{\mathrm{U}}$ than the neutral and unstable cases.

The minus five-thirds law is followed above about $n z / \overline{\mathrm{U}}=1.0$ for the vertical component spectra and extends to as low as $n z / \bar{U}=0.2$ for the longitudinal component spectra for unstable cases, to 0.4 for the neutral cases, while the stable tests agreed only above $n z / \bar{U}=1.0$. However, the cospectra became zero only above $\mathrm{nz} / \overline{\mathrm{U}}=1.0$ to 3.0 . This limitation on the inertial subrange has not generally been experimentally determined. The lateral component spectra for the 3.0 and 6.1 meter levels failed to agree with the minus five-thirds law within the frequency range of analysis except in two cases. where it was observed above $n z / \bar{U}=3.0$. At greater helghts the $v^{\prime}$ results are again inconclusive. The light wind speed unstable test at 12.2 meters follows the minus five-thirds law beyond about 1.0 but the other unstable test at 12.2 meters does not fit the minus 
five-thirds law well. The 87 meter case fits possibly only beyond $n z / \overline{\mathrm{U}}=10$.

Evaluation of the universal constant, $a$, for the inertial subrange relationship led to results, on the average, consistent with previous evaluations generally around $a=0.138$. However, the results from the $u^{\prime}$ component spectra led to a value about $20 \%$ too large and the vertical component spectra led to values about 10\% low. The two lateral component spectra from which evaluations could be made gave the expected result.

Dependence of the inertial subrange on $\varepsilon^{2 / 3}$ was also quite well established through the agreement between the measured power spectra and the universal normalized expression for the subrange, Equation (6.3b).

Reynolds' stress calculations show the measured friction velocity to be consistent with independently measured wind speeds. Ratios of $\sigma_{w} / u^{*}$ were found for neutral conditions to be quite constant at about 1.33. Unstable test ratios agree with this value but stable tests offer somewhat higher ratios. The ratios of $\sigma_{u} / u^{\star}$ and $\sigma_{v} / u^{*}$, averaging 2.9 and 2.0 respectively for neutral conditions show no obvious height, stability or wind speed dependence but the values vary somewhat more than the $\sigma_{w} / u^{*}$ ratios.

In treating the data some definite advantages in the analog analysis techniques have been noted. The analog computer treats the turbulence signals continuously, avoiding 
the necessity of discrete sampling and simplifying the spectral analysis of turbulence at high frequencies. The analog computer is easily proqrammed to provide the variety of analyses necessary and when changes in the programs are required they can be made easily and tested on the data immediately with little interruption in the analysis. Any point in the analysis can be monitored, supplying information which can suggest program changes to improve the analysis, revealing deficiencies in the data which might have been easily overlooked at the original measurement, and providing insights into the statistical reliability of the data. Disadvantages in the analog analysis techniques include the considerable time required for analysis and the limitations on the accuracy of the results. The results of the present investigation are estimated as being accurate to within 3 to 58 in the coordinate transformation and to within 5 to 108 in the spectral analysis, independent of the statistical variability. At the low frequency end of the spectra the 808 confidence interval only occasionally falls within \pm 50 of the true average value but at the center of the range of frequencies analyzed the variations in the estimates fall within $\$ 308$ of the true average value 808 of the time for almost all tests. Estimates at the highest frequency of analysis for all tests are within 178 of the average value 808 of the time, generally being within 
the limits of accuracy of the analysis technique. The improved defintion of the spectra at high frequencies due to increased stability of the estimates was another advantage of the analog analysis. Because of the large statistical variability at low wave numbers the spectra for each component within each stability group were averaged, considerably improving the reliability of the spectral distributions.

Future investigations will include more measurements of the kind reported here. Dependence of spectral characteristics on height and on stability will be further studied. Continued measurement of spectral and cospectral distributions for the three wind components will be extended to several other heights of measurement and to more extreme stabilities. The variation of Reynolds' stress with stability should also be clarified in future tests with measurements in more extreme stabilities.

Measurements of temperature fluctuations will be required to determine precisely stability as indicated by $z / L$ or the flux Richardson Number. Furthermore, such temperature measurements, leading to turbulent heat flux determinations, and additional turbulent wind component measurements simultaneously taken at a number of heights, leading to an appraisal of the flux divergence of turbulent energy, can provide a more critical evaluation of the turbulent energy budget equation and its relation to the 
form of the spectra.

Instrument comparison studies are also planned for the future. Comparison of the Hanford wind component meter with M. Miyake's instrument should be made, identifying the advantages of each. Comparison with a sonic anemometer is also desirable.

The Hanford Meteorological tower provides the opportunity for measuring not only spectra at many heights through much of the turbulent boundary layer but also the change in momentum flux and heat flux with height under various stability conditions. The aircraft operated by Battelle-Northwest for meteorological studies also offers opportunities for future comparison of aircraft measured spectra with tower mounted wind component meter spectra. The effort directed toward diffusion studies permits the experimental investigation of turbulence and diffusion jointly. Experiments with extensive measurements of turbulence and the resulting turbulent diffusion are planned for the future at Hanford. Much insight remains to be gained on the problem of diffusion and its relation, to the turbulent structure of the atmosphere, particularly in stable conditions (see stewart ${ }^{42}$ ). Both theoretical and experimental efforts are required in this direction. 
REFERENCES

1. Batchelor, G. K. (1956). The theory of homogeneous turbulence. The University Press, Cambridae, England.

2. Berman, S. (1965). Estimating the longitudinal wind spectrum near the ground. Quart. J. Roy. Meteorol. Soc., 91, p. 302 .

3. Blackman, R. B. and J. W. Tukey (1958). The measurement of power spectra. Dover, New York.

4. Businger, J. A. and V. E. Suomi (1958). Variance spectra of the vertical wind component derived from observations of the sonic anemometer at O'Neill, Nebraska, in 1953. Archiv fur Meteor. und Klimat., 10, p. 415.

5. Calder, K. L. (1949). The criterion of turbulence in a fluid of variable density with particular reference to conditions in the atmosphere. Quart. J. Roy Meteorol. Soc., 75, pp. 71-78.

6. Cramer, H. E., et al. (1962). Studies of the spectra of the vertical fluxes of momentum, heat, and moisture in the atmospheric boundary layer. Final Rep., Contract No. DA-36-039-SC-80209. Dept. of Meteor., Mass. Inst. of Tech.

7. Cramer, H. E. (1959). Measurements of turbulence structure near the ground within the frequency range from 0.5 to 0.01 cycles sec. ${ }^{-1}$ Advances in Geophysics, $6, p .75$. $^{2}$

8. Cummins, J. C. (1960). Frequency spectrum of Calder Hall reactor noise. Rep. AEEW-M-19. United Kingdom 
Atomic Energy Authority, Research Group, Winfrith, Dorset, England.

9. Davenport, A. G. (1961). The spectrum of horizontal gustiness near the ground in high winds. Quart. J. Roy. Meteorol. Soc., 87, p. 194.

10. Ellison, T. H. (1957). Turbulent transport of heat and momentum from an infinite rough plane. J. Fluid Mech., 2 , p. 456 .

11. Ely, R. P., Jr. (1958). Spectral analysis of the u-component of wind velocity at three neters. J. Meteorol.' 15. p. 196 .

12. Favre, A., J. Gaviglio and R. Dumas (1958). Further space-time correlations of velocity in a turbulent boundary layer. J. Fluid Mech., 3, p. 344 .

13. Gifford, F., Jr. (1959). The interpretation of meteorological spectra and correlations. J. Meteorol., 16, p. 344 .

14. Gill, G. C. (1954). A fast response anemometer for micrometeorological investiqation, Bulletin A.M.S., 35.

15. Gurvich, A. S. (1960). Frequency spectra and distribution functions of vertical wind components. Izvestia ANSSSR, Geophys. Ser. 1960, No. 7, p. 1042.

16. Johnson, C. I. (1956). Analog computer technigues. McGraw-Hill, New York.

17. Kolmogoroff, A. N. (1941). The local structure of turbulence in incompressible viscous fluid for very 
large Reynolds numbers. Doklady ANSSSR, 30, p. 301.

18. Korn, G. A. and T. M. Korn (1956). Electronic analog computers. McGraw-Hill,

19. Lee, Y. W. (1960). Statistical theory of communications. John Wiley, New York.

20. Lumley, J. L. and H. A. Panofsky (1964). The structure of atmospheric turbulence. John Wiley, New York.

21. MacCready, P. B., Jr. (1953). Structure of atmospheric turbulence. J. Meteorol., 10, p. 434.

22. MacCready, P. B., Jr. (1962). The inertial subrange of atmospheric turbulence. J. Geophys. Research, 67, p. 1051 .

23. MacCready, P. B., Jr. (1962). Turbulence measurements by sailplane. J. Geophys. Research, 67, p. 1041 .

24. Miyake, M. (1965). A constant temperature wind component meter development and application. Final Rep., Contract No. AT (45-1)-1545. Dept. of Atmos. Sci., Univ. of washington, Seattle.

25. Monin, A. S. (1959). Smoke propagation in the surface layer of the atmosphere. Advances in Geophysics, 6, p.331. 26. Monin, A. S. (1959). On the similarity of turbulence in the presence of a mean vertical temperature gradient. I. Geo. Res., 64, p. 2196 .

27. Monin, A. S. (1962). Empirical data on turbulence in the surface layer of the atmosphere. J. Geophys. Research, 67 , p. 3103

28. Monin, A. S. and A. M. Oboukhov (1954). Basic regularity 
in turbulent mixing in the surface layer of the atmosphere. Trudy Geophys. Inst. ANSSSR, No. 24, p. 163.

29. Panofsky, H. A. (1961). An alternative derivation of the diabatic wind profile. Quart. J. Roy. Meteorol. Soc.; 87 , p. 109 .

30. Panofsky, H. A. (1962). The budget of turbulent energy in the lowest 100 meters. J. Geophys. Research, 67, p. 3161 .

31. Panofsky, H. A. (1953). The variation of the turbulence spectrum with height under superadiabatic conditions. Quart. J. Roy. Meteorol. Soc., 79, p. 150.

32. Panofsky, H. A., H. E. Cramer and V. R. K. Rao (1958). The relation between Eulerian time and space spectra. Quart. J. Roy. Meteorol. Soc., 84, p. 270.

33. Panofsky, H. A. and R. J. Deland (1959). One-dimensional spectra of atmospheric turbulence in the lowest 100 metres. Advances in Geophysics, 6, p. 41 .

34. Panofsky, H. A, and R. A. McCormick (1960). The spectrum of vertical velocity near the surface. Quart J. Roy. Meteorol. Soc., 86, p. 495.

35. Panofsky, H. A. and H. Press (1962). Meteorological and aeronautical aspects of atmospheric turbulence. Prog. in Aero. Sci., 3, p. 179. Pergamon Press.

36. Panofsky, H. A. and I. Van der Hoven (1956). Structure of small scale and middle scale turbulence at Brookhaven. Sci. Rep. No. 1, Contract No. AF 19(604)-1027. Pennsylvania State University. 
37. Pasquill, F. (1962). Recent broad-band spectral measurements of turbulence in the lower atmosphere. J. Geophys. Research, 67 , p. 3025 .

38. Pond, S., R. W. Stewart and R. W. Burling (1963). Turbulence spectra in wind over waves. J. Atmosph. Sci., 20 , p. 319 .

39. Priestley, C. H. B. (1959). Turbulent transfer in the lower atmosphere. University of Chicago Press, Chicago.

40. Ratcliffe, C. A. and E. M. Sheen (1964). Wind component analyzer improvements. Unpublished report. General Electric Co., H.A.P.O., Richland, Washington.

41. Ratcliffe, C. A. and E. M. Sheen (1964). An automatic data collection system for meteorological tower instrumentation. J. Appl. Meteorol., 3, p. 807.

42. Stewart, R. W. (1959). The problem of diffusion in a stratified fluid. Advances in Geophysics, 6, p. 303 .

43. Taylor, G. I. (1938). The spectrum of turbulence. Proc. Roy. Soc., Al64, p. 476.

44. Webb, E. R. (1955). Autocorrelations and energy spectra of atmospheric turbulence. C.S.I.R.O. Div. Met. Phys. Tech. Pap. No. 5. Melbourne.

45. Zubkovski, S. L. (1962). Frequency spectra of the horizontal wind-speed component close to the ground. Izvestia ANSSSR, Geophys. Ser. 1962, No. 10, p. 1425. 


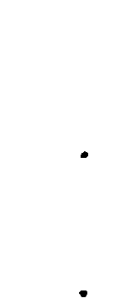


APPENDIX A

For an aperiodic or transient function of time, $f(t)$, where $\int_{-\infty}^{\infty} f(t) d t$ is finite, the Fourier integral representation is given by

$$
f(t)=\int_{-\infty}^{\infty} F(\omega) e^{i \omega t} d_{\omega}
$$

where

$$
F(\omega)=\frac{1}{2 \pi} \int_{-\infty}^{\infty} f(t) e^{-i \omega t} d t
$$

Equations (A.1) and (A.2) form a Fourier transform pair. The complex continuous spectrum, $F(\omega)$, of the aperiodic function, $f(t)$, is a complex quantity, i.e.

$$
F(\omega)=P(\omega)+i L(\omega)
$$

We can see that $F(\omega)$ is indeed the continuous spectrum of $f(t)$ from Equation (A.I) which states that $f(t)$ is composed of an infinite number of sinusoids, $e^{i \omega t}$, over a continuous infinite range of frequencies, each with an infinitesimal amplitude $F(\omega)$ dw.

Considering, now, two aperiodic functions $f_{1}(t)$ and $f_{2}(t)$ with respective spectrum functions $F_{1}(w)$ and $F_{2}(w)$, we define the covariance between them as 


$$
\phi_{12}(\tau)=\int_{-\infty}^{\infty} f_{1}(t) f_{2}(t+\tau) d t
$$

Through the Fourier integral representations of $f_{1}(t)$ and $f_{2}(t)$, this can be re-expressed as

$$
\begin{aligned}
\phi_{12}(\tau) & =\int_{-\infty}^{\infty} 2 \pi \bar{F}_{1}(\omega) F_{2}(\omega) e^{i \omega t} d_{\omega} \\
& =\int_{-\infty}^{\infty} 12(\omega) e^{i \omega \tau} d_{\omega}
\end{aligned}
$$

where $\bar{F}_{1}(\omega)$ represents the complex conjugate of $F_{1}(\omega)$ and where we have defined the spectral density function as

$$
12(\omega)=2 \pi F_{1}(\omega) F_{2}(\omega)
$$

We see from $(A .4)$ that $\phi_{12}(\tau)$ is the Fourier transform of $\Phi_{12}(\omega)$ so that the inverse relationship must hold, i.e.

$$
\Phi_{12}(\omega)=\frac{1}{2 \pi} \int_{-\infty}^{\infty} \rho_{12}(\tau) e^{-i \omega \tau} d \tau
$$

When both aperiodic functions are the same, Equation (A.3) describes the autocovariance function for an aperiodic function.

$$
11(x)=\int_{-\infty}^{\infty} f_{1}(t) \quad f_{1}(t+\tau) d t
$$

With the energy density spectrum,

$$
\Phi_{21}(\omega)=2 \pi\left|F_{1}(\omega)\right|^{2}
$$


the autocovariance function forms a Fourier transform pair,

$$
\begin{aligned}
& \phi_{12}(\tau)=\int_{-\infty}^{\infty} \Phi_{11}(\omega) e^{i \omega \tau} d_{\omega} \\
& \phi_{11}(\omega)=\frac{1}{2 \pi} \int_{-\infty}^{\infty} \phi_{11}(\tau) e^{-i \omega \tau} d \tau
\end{aligned}
$$

and

For $\tau=0$, Equation (A.9) becomes

$$
\phi_{11}(0)=\int_{-\infty}^{\infty} \Phi_{11}(\omega) d \omega
$$

demonstrating that the total energy, $\phi_{11}(0)=\int_{-\infty}^{\infty} f_{1}(t)^{2} d t$, is distrubted over frequency as described by the energy density spectrum. This is Parseval's equation.

When the aperiodic functions in (A.3) are different functions of time, the expression is termed the crosscovariance and its transform is called the cross-spectral density function which, since the crosscovariance is not necessarily an even function, is seen to be a complex quantity,

$$
\$_{12}(w)=C_{12}(w)+i Q_{12}(w)
$$

The real part of the cross spectral density function is termed the cospectral density, and the imaginary part the quadrature spectral density.

From (A.4), the total covariance is

$$
\phi_{12}(0)=\int_{-\infty}^{\infty} f_{1}(t) f_{2}(t) d t=\int_{-\infty}^{\infty} \phi_{12}(\omega) d \omega \cdot(A \cdot 13)
$$


Before proceeding to a discussion of random continuous functions, it is appropriate to mention at this point the convolution integral which will be used later with a discussion of truncated random functions. Consider again, two aperiodic functions of time, $f_{1}(t)$ and $f_{2}(t)$, each with its respective transform $F_{1}(w)$ and $F_{2}(w)$, with which transform pairs such as (A.I) and (A.2) can be written. The Fourier transform of the product of the two time functions is

$$
F(\omega)=\frac{1}{2 \pi} \quad \int_{-\infty}^{\infty}\left[f_{1}(t) f_{2}(t)\right] e^{-i \omega t} d t \quad(A .14)
$$

Expressing $f_{2}(t)$ with its spectral representation, reversing the resulting order of integration and substituting the Fourier transform of $f_{1}(t)$ evaluated at the frequency $(\omega-\xi)$, we obtain the convolution integral,

$$
F(\omega)=\int_{-\infty}^{\infty} F_{1}(\omega-\xi) F_{2}(\xi) d \xi
$$

expressing the transform of a product of two aperiodic function in terms of the individual transforms of the functions. This is often written symbolically as

$$
F(\omega)=F_{1}(\omega) \star F_{2}(\omega)
$$

From (A.15) we can see that the convolution integral gives us a view of one function of frequency, altered by the shape of the second function centered at some given frequency, . Turning now to random continuing functions, we 


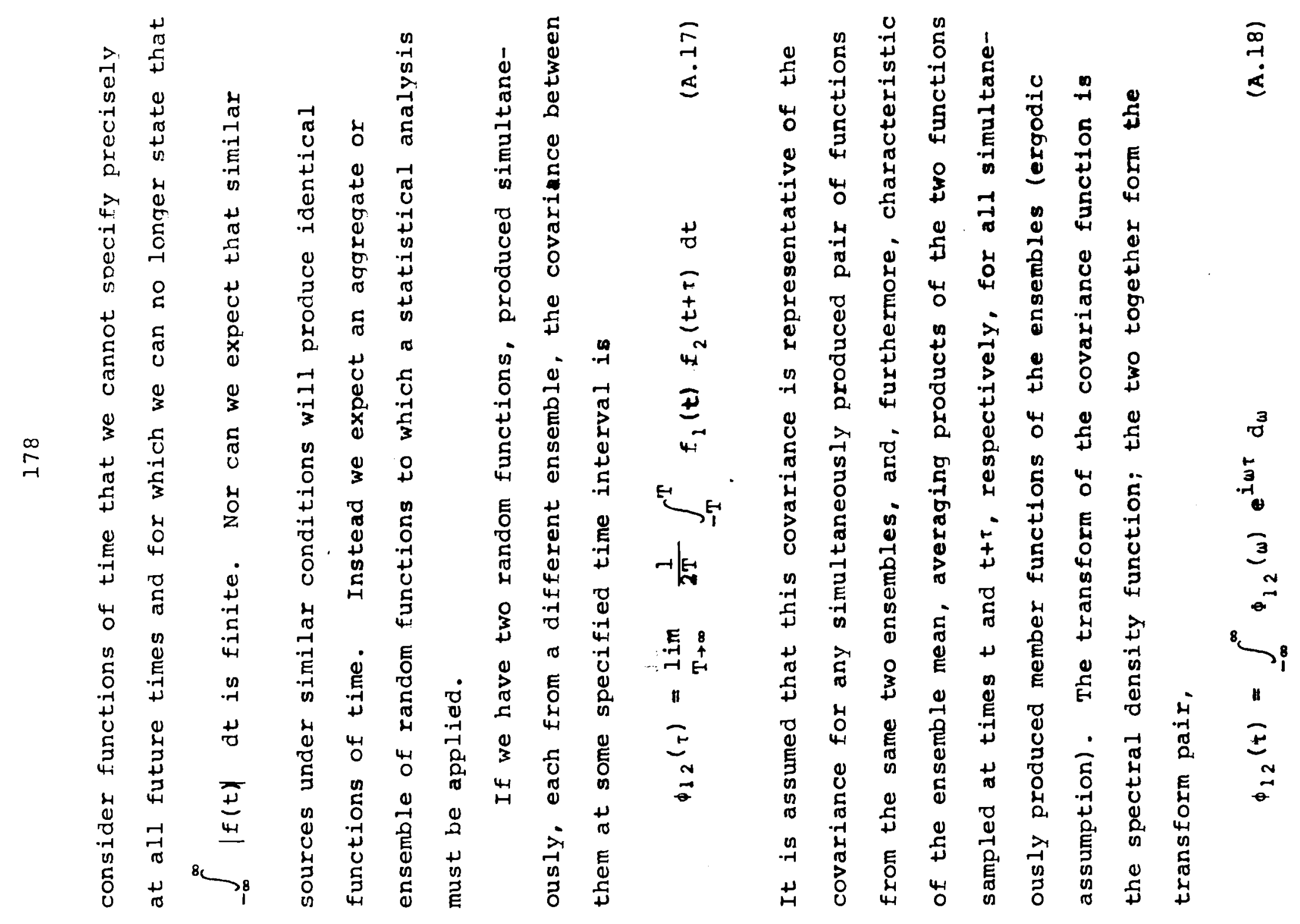




$$
\Phi_{12}(\omega)=\frac{1}{2 \pi} \quad \int_{-\infty}^{\infty} \phi_{12}(\tau) e^{-i \omega \tau} d \tau
$$

When the two random functions are the same, Equation (A.17) becomes the autocovariance,

$$
\phi_{11}(\tau)=\lim _{T \rightarrow \infty} \frac{1}{2 T} \int_{T}^{T} f_{1}(t) f_{1}(t+\tau) d t
$$

whose transform is the power spectral density,

$$
\Phi_{11}(\omega)=\frac{1}{2 \pi} \int_{-\infty}^{\infty} \phi_{11}(\tau) e^{-i \omega \tau} d \tau
$$

Again, for $t=0$, Equations (A.20) and (A.22) demonstrate how the average power is distributed over frequency according to the power spectral density function,

$$
\lim _{T+\infty} \frac{1}{2 T} \int_{-T}^{T} f_{1}^{2}(t) d t=\int_{-\infty}^{\infty} \Phi_{11}(\omega) d \omega
$$

When the random functions of time are different, Equation (A.17) is called the cross-covariance and its transform is termed the cross-spectral density. Since the cross-covariance is not necessarily an even function (as opposed to the autocovariance function), the cross spectrum is generally complex, the real part being the cospectrum and the imaginary part, the quadrature spectrum, i.e.

$$
\Phi_{12}(w)=c_{12}(w)+i Q_{12}(w)
$$


For $\tau=0$, the average covariance is seen from Fquation (A.17) and (A.18) to be the integral of contributions over the entire range of frequency

$$
\lim _{T \rightarrow \infty} \frac{1}{2 T} \int_{-T}^{T} f_{1}(t) f_{2}(t) d t=\int_{-\infty}^{\infty} \Phi_{12}(\omega) d_{\omega}
$$

When experimental spectral distributions are determined with digital analysis methods, Equations (A.17) through (A.25) are generally used, determining covariance at various laq intervals from the data and taking transforms of the resulting covariance functions. However, since the data is necessarily limited in length of record, the covariance for all lag intervals cannot be determined and consequently neither can the integral over an infinite range of lags, necessary for the transformation to the spectral density function. Blackman and Tukey ${ }^{3}$ have demonstrated how the spectral estimates obtained are actually convolutions of the true spectrum with a "spectral window," the transform of some "lag window" which alters the covariance function. The lag window, which is multiplied with the covariance function, must at least be a rectangular function equal to one over a limited continuous range of lag time and zero elsewhere, simply because of the limited length of record. However, it can arbitrarily be selected in the analysis in order to optimize the type of spectral estimate desired, which is actually an area under the spectral density function curve 
in the region of some frequency, $w$, described by the convolution integral

$$
\operatorname{av}[\hat{\Phi}(\omega)]=H(\omega) \star \phi(\omega)
$$

where av $\left[\begin{array}{ll}\hat{\phi} & (\omega)]\end{array}\right.$ is the ensemble average of the measured spectral estimates at a given frequency and $H(w)$ is the spectral window or power transfer function. In addition to producing spectral estimates containing contributions from the spectral density distribution over a range of frequency, the use of a finite length of record, or a sample of the true time series, also produces the statistical variability that accompanies any sampling process.

An equally valid, but less frequently used, technique for analyzing spectral content of turbulence data is the filtering method. This can be performed either electrically or numerically. However, the following discussion will be directed toward electrical filtering, the means by which the spectral analysis of the present study was carried out.

A filter is a linear system characterized by its response to a unit impulse function. The unit impulse is an even function of infinitesimal width and unit area. With this as an input to a linear system its output is the unit impulse response. The filtering of a continuous time series, $f(t)$, can be considered as a convolution of the unit impulse response, $w(t)$, with the time series so that the output of the filter can be written as 


$$
f_{0}(t)=\int_{-\infty}^{\infty} w(t-\lambda) f_{1}(\lambda) d \lambda
$$

or equivalently

$$
f_{0}(t)=\int_{-\infty}^{\infty} w(\xi) f_{1}(t-\xi) d \xi
$$

For a time series with a zero mean value, the autocovariance of the output signal is

$$
\phi_{00}(\tau)=\lim _{T \rightarrow \infty} \frac{1}{T} \int_{-T / 2}^{T / 2} f_{0}(t) f_{0}(t+\tau) d t
$$

which, after introducing (A.28) and interchanging the order of integration, becomes

$$
\phi_{00}(\tau)=\int_{-\infty}^{\infty} \int_{-\infty}^{\infty} W\left(\xi_{1}\right) W\left(\xi_{2}\right) \phi_{11}\left(\tau+\xi_{1}-\xi_{2}\right) d \xi_{1} d \xi_{2}
$$

Expressing the autocovariance in the integral above as the Fourier transform of the spectral density function, given by (A.19), and interchanging the order of integration once again, Equation (A.29) becomes

$$
\begin{aligned}
\phi_{00}(\tau) & =\int_{-\infty}^{\infty} Y(\omega) \bar{Y}(\omega) \Phi_{11}(\omega) e^{i \omega \tau} d \omega \\
& =\int_{-\infty}^{\infty}|Y(\omega)|^{2} e_{1}(\omega) e^{i \omega \tau} d \omega
\end{aligned}
$$

where the system or transfer function, $Y(w)$, is the transform of $w(t)$,

$$
Y(\omega)=\int_{-\infty}^{\infty} w(t) e^{-i \omega t} d t
$$


and is also the ratio of the input and output complex magnitudes for a steady state sinusoidal input to the linear system. Its conjugate is $\bar{Y}(\omega)$. For $\tau=0$, Equation $(A .30)$ becomes

$$
\overline{f_{0}{ }^{2}}(t)=\phi_{00}(0)=\int_{-\infty}^{\infty}|Y(\omega)|^{2} \Phi_{11}(\omega) d \omega
$$

For filtering two time series to determine the cospectral density distribution, each signal is passed through a separate filter. Considering that the two filters are identical, the outputs are convolutions of the unit response function with the original time series,

$$
\begin{aligned}
& f_{01}(t)=\int_{-\infty}^{\infty} w\left(\xi_{1}\right) f_{1}\left(t-\xi_{1}\right) d \xi_{1} \\
& f_{02}(t)=\int_{-\infty}^{\infty} w\left(\xi_{2}\right) f_{2}\left(t-\xi_{2}\right) d \xi_{2}
\end{aligned}
$$

Following the same procedure as with the autocovariance, the cross covariance function for the output is shown to be

$$
\phi_{012}(\tau)=\int_{-\infty}^{\infty}|Y(\omega)|^{2} \Phi_{12}(\omega) e^{i \omega \tau} d_{\omega}
$$

Again, for $\tau=0$

$$
\overline{F_{01}(t) f_{02}(t)}=\int_{-\infty}^{\infty}|Y(w)|^{2} \varphi_{12}(w) d \omega
$$

and since this is a real quantity only, the mean output product is a portion of the area under the cospectral 
density function curve, defined by the shape of the filter transfer function, or

$$
\overline{f_{01}(t) f_{02}(t)}=\int_{-\infty}^{\infty}|Y(\omega)|^{2} \quad c_{12}(\omega) d \omega
$$

Up to this point, the estimation of spectral density through the limiting form of the Fourier transform of the random time series has not been considered. The same difficulties arise with this direct approach as those discussed by Blackman and Tukey when the transform of the limiting form of the autocovariance is used. As we take the transform of longer and longer records of the time series, how closely we are approaching the limiting form of the transform is not known; indeed, even though it may be approached on the average, the variance of the estimates may become infinite. Instead of considering the limiting form, we may consider, using Equations (A.1) through (A.13), the Fourier transform of a truncated time series, a transient function, which is actually what we have in our limited record. Blackman and Tukey describe this as modifying the data with a "data window." In fact, we may wish to limit or alter the record even beyond what has been imposed on it by the limited sampling capabilities, e.g. the original record may be passed through a filter. In this case, the time series can be thought of as being altered by some weighting function, $w(t)$, such that 


$$
\int_{-\infty}^{\infty} W(r) f(t-r) d r \text { is finite. }
$$

The Fourier transform of the transient function representing this sample of the time series is

$$
\hat{F}_{j}(\omega, t)=\int_{-\infty}^{\infty} w(r) f_{j}(t-r) e^{-i \omega r} d r
$$

When the original time series is multiplied by a sine function and by a cosine function for some fixed frequency, $\omega$, and each product is passed through a filter, the outputs are the real and imaginary parts of Equation (A.38) at some instant, $t$. For a second time series treated in the same manner, the filter outputs at some instant, $t$, are the real and imaginary components of the sample Fourier transform

$$
\hat{F}_{k}(\omega, t)=\int_{-\infty}^{\infty} w(s) f_{k}(t-s) e^{-i \omega s} d s
$$

Taking the product of Equation (A.38) with the conjugate of Equation (A.39) and then averaging, in this case over all the sample products being produced continuously as a function of time by the filters, we have, after changing the order of integration

$$
\begin{aligned}
\lim _{T \rightarrow \infty} & \frac{1}{T} \int_{-T / 2}^{T / 2} \hat{F}_{j}(\omega, t) \hat{\bar{F}}_{k}(\omega, t) d t=\hat{\Phi}_{j k}(\omega) \\
& =\int_{-\infty}^{\infty} D(\tau) \phi_{j k}(\tau) e^{-i \omega \tau} d \tau
\end{aligned}
$$

where $\hat{~}_{j k}(\omega)$ is the measured spectral density and $D(\tau)$, for identical filters, is the autocovariance of the unit response 
function of the filter, $W(t)$. Then, from the convolution theorem we know that the measured spectral density is

$$
\hat{\Phi}_{j k}(\omega)=H(\omega) \star \Phi_{j k}(\omega)=\int_{-\infty}^{\infty} H(\omega-\xi) \Phi_{j k}(\xi) d \xi
$$

where $H(w)$ is the power transfer function or spectral window, the transform of $D(\tau)$, and $\Phi_{j k}(\omega)$ is again the true spectral density. Thus, the technique described above for obtaining spectral estimates by the direct method, starting with the Fourier transform of a truncation of the original time series yields results similar to those obtained with the indirect method where the transform of the covariance function for records of finite length is used to obtain spectral estimates. 


\section{ACKNOWLEDGMENTS}

To Professor Franklin I. Badgley, chairman of my thesis supervisory committee, goes my deepest appreciation for his patience, friendliness, and encouragement as well as for the technical insight he offered in guiding my research efforts.

Professor H. A. Panofsky was most helpful in reviewing the study, offering enlightening suggestions, and relating recent work by other investigators not yet published.

To Mr. James J. Fuquay, who collaborated at Hanford on this study, I am most thankful. Without his initial developmental work on the wind component meter, this study would not have been possible. As my supervisor at Hanford, he gave impetus to the work and a freedom of research enabling the study to proceed unimpeded.

My sincerest appreciation goes to the staff of the Atmospheric Sciences Section of Battelle-Northwest who helped immeasurably in the field and data reduction work. Mr. Paul Nickola offered his cheerful assistance in providing mean wind and temperature profile data. Mr. J. W. Sloot, Mr. R. L. Conley, Mr. B. N. Nelson, and Mr. D. M. Hughey aided in the data reduction most conscientiously.

Thanks go to Mr. Paul Dionne and Mr. J. Draper of the analog computer facility at Hanford who were most helpful and conscientious in programming and operating the analog computer during the tedious data analysis period. 
Number of Copies
Air Force Cambridge Research Laboratories

D. A. Haugen

J. C. Kaimal

Atomic Energy Commission, Washington Division of Biology and Medicine

J. 2. Holland

Bedford Institute of Oceanography P. O. Box 1006 Dartmouth, N. W. Canada

S. D. Smith

Booz Allen Applied Research Incorporated 6151 West Century

Los Angeles, California 90045

R. Cale

Division of Technical Information Extension

New York University

Geophysical Science Laboratory

2455 Sedgwick Ave.

Bronx, New York

A. D. Kirwan, Jr.

Pennsylvania State University

Department of Meteorology

University Park, Penn,

H. A. Panofsky

Richland Operations Office

R. K. Sharp

Technical Information Library

Travelers Research Center, Inc. Hartford Connecticut

G. R. Hilst 
Number of Copies

1

University of British Columbia Institute of Oceanography

Vancouver, British Columbia

R. W. Burling

112

Batte1le-Northwest

G. M. Dalen

P. J. Dionne

C. E. Elderkin (100)

J. J. Fuquay

R. S. Paul

C. L. Simpson

Technical Information Files

Technical Publications (2) 